Supporting Information

for

\title{
On Origin of Kinetic Resolution of Hydroxy Esters through Catalytic Enantioselective Lactonization by Chiral Phosphoric Acids
}

Avtar Changotra and Raghavan B. Sunoj*

\author{
Department of Chemistry \\ Indian Institute of Technology Bombay \\ Powai, Mumbai 400076
}

Email: sunoj@,chem.iitb.ac.in 


\begin{tabular}{|c|c|c|}
\hline Page No. & & Table of Contents \\
\hline & & Computational Methods \\
\hline S4 & Table S1 & $\begin{array}{l}\text { Computed relative distortion energies in each fragment, total } \\
\text { relative distortion energies }\left(\Delta \mathrm{E}_{\mathrm{d}}^{+}\right) \text {and relative interaction } \\
\text { energies }\left(\Delta \mathrm{E}^{\ddagger}\right) \text { in the stereocontrolling TSs at the M06-2X/6- } \\
31 \mathrm{G}^{* *} \text { level of theory for both the pathways }\end{array}$ \\
\hline S5 & Table S2 & $\begin{array}{l}\text { Computed relative distortion energies in each fragment, total } \\
\text { relative distortion energies }\left(\Delta \mathrm{E}_{\mathrm{d}}^{+}\right) \text {and relative interaction } \\
\text { energies }\left(\Delta \mathrm{E}_{\mathrm{i}}^{*}\right) \text { of the initial recognition complex in the } \\
\text { associated with the stereocontrolling TSs at the M06-2X/6-31G** } \\
\text { level of theory for both the enantiomers in the stepwise pathway }\end{array}$ \\
\hline S5-S6 & Figure S1 & $\begin{array}{l}\text { Overlaid Images of the substrate and the catalyst as seen in the } \\
\text { initial recognition complex associated with the stereocontrolling } \\
\text { TSs for both the } S \text { and } R \text { enantiomers in the stepwise pathway }\end{array}$ \\
\hline S7 & Figure S2 & $\begin{array}{l}\text { The intrinsic reaction coordinate (IRC) trajectory for the lowest } \\
\text { energy transition state }[3-4]_{S \text { si anti }}^{*}\end{array}$ \\
\hline S8-S9 & Figure S3 & $\begin{array}{l}\text { Different possible conformers of transition states for the } S \text { and } R \\
\text { enantiomers in the concerted pathway }\end{array}$ \\
\hline S9 & Table S3 & $\begin{array}{l}\text { The relative Gibbs free energies (in } \mathrm{kcal} / \mathrm{mol} \text { ) of } \mathrm{C}-\mathrm{O} \text { bond } \\
\text { formation with respect to the lowest energy transition state in the } \\
\text { concerted pathway }\end{array}$ \\
\hline S9 & Figure S4 & $\begin{array}{l}\text { The optimized geometries of the lowest energy diastereomeric } \\
\text { transition states at the M06-2X/6-31G** level of theory for the } \\
\text { stereoselective } \mathrm{C}-\mathrm{O} \text { bond formation in the concerted pathway }\end{array}$ \\
\hline S10-S11 & Table S4 & $\begin{array}{l}\text { The relative Gibbs free energies (in } \mathrm{kcal} / \mathrm{mol} \text { ) of } \mathrm{C}-\mathrm{O} \text { bond } \\
\text { formation with respect to the lowest energy transition state in the } \\
\text { stepwise pathway }\end{array}$ \\
\hline S11 & Figure S5 & $\begin{array}{l}\text { Wiberg bond indices for selected points along the IRC trajectory } \\
\text { for }[6-7]_{S \_s i s y n}\end{array}$ \\
\hline S12 & Figure S6 & Gibbs free energy profile \\
\hline S12-S13 & & Enantiomeric excess of unreacted $\alpha$-methyl $\gamma$-hydroxy ester \\
\hline S13-S14 & Table S5 & $\begin{array}{l}\text { Activation strain analysis on the stereocontrolling TSs at the } \\
\text { M06-2X/6-31G** level of theory in the stepwise pathway }\end{array}$ \\
\hline $\mathrm{S} 14-\mathrm{S} 15$ & Figure S7 & $\begin{array}{l}\text { The topological analysis of the electron densities obtained using } \\
\text { the Atoms-In-Molecule formalism for the stereocontrolling } \\
\text { transition states }\end{array}$ \\
\hline S15 & Table S6 & $\begin{array}{l}\text { Summary of electron densities }\left(\rho_{\mathrm{bcp}}\right) \text { at the bond critical points } \\
\text { along the bond paths in the stereocontrolling transition states }\end{array}$ \\
\hline S15-16 & Figure S8 & Space-filling models of TSs for the $\mathrm{C}-\mathrm{O}$ Bond Formation \\
\hline S16-S17 & Figure S9 & The optimized geometries of transition states \\
\hline S18 & Figure S10 & $\begin{array}{l}\text { The optimized geometries of diastereomeric transition states at } \\
\text { the M06-2X/6-31G** level of theory for the removal of }{ }^{t} \mathrm{BuOH}\end{array}$ \\
\hline \multicolumn{2}{|c|}{ S19-S190 } & $\begin{array}{l}\text { The optimized coordinates of all the stationary points in the gas } \\
\text { phase involved in the formation of lactone product }\end{array}$ \\
\hline
\end{tabular}




\section{Computational Methods}

All calculations were performed with Gaussian09 Revision D.01 suite of quantum chemical program. ${ }^{1}$ Geometries were fully optimized and characterized by frequency calculation using the M06-2X $\mathrm{X}^{2}$ density functional theory (DFT) with Pople's $6-31 \mathrm{G}^{* *}$ basis set for all atoms. ${ }^{3}$ Gibbs free energies $(298.15 \mathrm{~K}, 1 \mathrm{~atm})$ were initially computed in the gas phase. The stationary points were characterized by frequency calculations. The transition states were verified by the unique imaginary frequency pertaining to the desired reaction coordinate. Intrinsic reaction coordinate (IRC) calculations were carried out to ascertain the correctness of the transition states obtained. ${ }^{4}$ The effect of a solvent continuum, in dichloromethane (DCM) $(\varepsilon=8.93$ for dichloromethane), was evaluated using the Cramer-Truhlar continuum solvation model that employs quantum mechanical charge density of solutes, designated as SMD. ${ }^{5}$ Single point energies were calculated at the $\mathrm{SMD}_{(\mathrm{DCM})} / \mathrm{M} 06-2 \mathrm{X} / 6-31 \mathrm{G}^{* * / / M 06-2 X / 6-~}$ $31 \mathrm{G}^{* *}$ level of theory. The free energies in dichloromethane (solvent continuum) were calculated from the sum of the single-point energies in dichloromethane and the value of thermal correction to Gibbs free energy in gas phase.

(1) Gaussian 09, Revision D.01, Frisch, M. J.; Trucks, G. W.; Schlegel, H. B.; Scuseria, G. E.; Robb, M. A.; Cheeseman, J. R.; Scalmani, G.; Barone, V.; Mennucci, B.; Petersson, G. A.; Nakatsuji, H.; Caricato, M.; Li, X.; Hratchian, H. P.; Izmaylov, A. F.; Bloino, J.; Zheng, G.; Sonnenberg, J. L.; Hada, M.; Ehara, M.; Toyota, K.; Fukuda, R.; Hasegawa, J.; Ishida, M.; Nakajima, T.; Honda, Y.; Kitao, O.; Nakai, H.; Vreven, T.; Montgomery, J. A., Jr.; Peralta, J. E.; Ogliaro, F.; Bearpark, M.; Heyd, J. J.; Brothers, E.; Kudin, K. N.; Staroverov, V. N.; Kobayashi, R.; Normand, J.; Raghavachari, K.; Rendell, A.; Burant, J. C.; Iyengar, S. S.; Tomasi, J.; Cossi, M.; Rega, N.; Millam, N. J.; Klene, M.; Knox, J. E.; Cross, J. B.; Bakken, V.; Adamo, C.; Jaramillo, J.; Gomperts, R.; Stratmann, R. E.; Yazyev, O.; Austin, A. J.; Cammi, R.; Pomelli, C.; Ochterski, J. W.; Martin, R. L.; Morokuma, K.; Zakrzewski, V. G.; Voth, G. A.; Salvador, P.; Dannenberg, J. J.; Dapprich, S.; Daniels, A. D.; Farkas, O.; Foresman, J. B.; Ortiz, J. V.; Cioslowski, J.; Fox, D. J. Gaussian, Inc., Wallingford CT, 2013. (2) Zhao, Y.; Truhlar, D. G. J. Phys. Chem. 2006, 125, 194101.

(3) (a) Hehre, W. J.; Ditchfield, R.; Pople, J. A. J. Chem. Phys. 1972, 56, 2257. (b) Hariharan, P. C.; Pople, J. A. Theor. Chim. Acta 1973, 28, 213. (c) Lee, C.; Yang, W.; Parr, R. G. Phys. Rev. B: Condens. Matter Mater. Phys. 1988, 37, 785. (d) Becke, A. D. J. Chem. Phys. 1993, 98, 5648.

(4) (a) Gonzalez, C.; Schlegel, H. B. J. Chem. Phys. 1989, 90, 2154. (b) Gonzalez, C.; Schlegel, H. B. J. Phys. Chem. 1990, 94, 5523.

(5) Marenich, A. V.; Cramer, C. J.; Truhlar, D. G. J. Phys. Chem. B 2009, 113, 6378. 


\section{Comparison of stepwise and concerted pathways by using Activation Strain analysis}

To gain insight into the origin of energy difference between the two pathways viz., concerted and stepwise, we employed the Activation Strain Analysis, where in $\Delta E^{\ddagger}$ (activation energy) is decomposed into distortion $\left(\Delta E_{\mathrm{d}}^{\ddagger}\right)$ and interaction energies $\left(\Delta E^{\dagger}{ }_{\mathrm{i}}^{\dagger} . \Delta E_{\mathrm{d}}^{\ddagger}\right.$ for the reactants is the energy difference between the original reactants and the corresponding distorted geometries in the respective TSs. $\Delta E_{\mathrm{d}}^{\ddagger}$ is the binding energy between the distorted reactants at the TS geometry. The distortion and interaction energies are then compared to the lowest energy transition state to obtain the relative distortion and interaction energies. To understand the origin of the energy difference between the concerted and the stepwise pathways, we have examined whether the catalyst BINOL phosphoric acid (treated as fragment $1, \mathrm{f1}$ ) or the substrate ( $\alpha$-methyl $\gamma$-hydroxy ester, treated as fragment $2, \mathrm{f} 2)$ is distorted more and whether such distortions have any bearing to the prediction that the concerted pathway is of higher energy. The computed distortion and interaction energies in the lower energy TS for both concerted and stepwise pathways are provided in Table S1.

Table S1. Computed Relative Distortion Energies (kcal/mol) in each Fragment, Total Relative Distortion Energies $\left(\Delta \mathrm{E}_{\mathrm{d}}^{\dagger}\right)$ and Relative Interaction Energies $\left(\Delta \mathrm{E}_{\mathrm{i}}^{\dagger}\right)$ with Respect to the Unstrained Geometries of the Substrates in the Stereocontrolling Transition States at the M06-2X/6-31G** Level of Theory for both the Pathways ${ }^{\mathrm{a}}$

\begin{tabular}{|c|c|c|c|c|c|}
\hline TS & $\Delta E_{\mathrm{d}}^{\ddagger}(\mathrm{f} 1)$ & $\Delta E_{\mathrm{d}}^{\ddagger}(\mathrm{f} 2)$ & $\begin{array}{c}\Delta E_{\mathrm{d}}^{\ddagger}(\text { total })= \\
\Delta E_{\mathrm{d}}^{\sharp}(\mathrm{f} 1)+\Delta E_{\mathrm{d}}^{\sharp}(\mathrm{f} 2)\end{array}$ & $\Delta E_{\mathrm{i}}^{*}$ & $\begin{array}{c}\Delta E^{\ddagger}= \\
{\left[\Delta E^{\ddagger}+\Delta E^{\ddagger}\right]}\end{array}$ \\
\hline$[3-4]_{S \_ \text {si_anti }}$ & 65.9 & 58.2 & 124.2 & -124.9 & -0.7 \\
\hline$[6-7]_{S \text { si syn }}{ }^{+}$ & 63.7 & 37.9 & 101.6 & -120.9 & -19.2 \\
\hline
\end{tabular}

${ }^{\mathrm{a}} \mathrm{f} 1$ and $\mathrm{f} 2$ are respectively the catalyst, BINOL phosphoric acid and the substrate $\alpha$-methyl $\gamma$-hydroxy ester

A quick perusal of distortion energies reveals that the contribution to the deformation energy is primarily due to the distortion in the substrate geometry. For instance, the substrate

in the concerted transition state $[\mathbf{3}-4]_{S_{-} \text {si_anti }}{ }^{\ddagger}$ is deformed by $20.3 \mathrm{kcal} / \mathrm{mol}$ more than that in 
$[6-7]_{S \_s i s s y n}{ }^{*}$ for cyclization in the stepwise pathway. At the same time, the difference in deformation within the catalyst structure between these two transition states are only 2.2 $\mathrm{kcal} / \mathrm{mol}$. Stabilizing interactions exhibited some interesting features. The higher energy concerted transition state $[3-4]_{S_{-} \_ \text {sianti }}{ }^{\ddagger}$ has $4.1 \mathrm{kcal} / \mathrm{mol}$ better stabilizing interaction than that in the lower energy $[6-7]_{S_{-} s i s y n}{ }^{*}$ involved in the stepwise pathway. Importantly, the net balance between the destabilizing distortion and stabilization interaction energies is found to be in favor of the ring closing transition state in the stepwise pathway, which is stabilized by $18.5 \mathrm{kcal} / \mathrm{mol}$.

\section{Comparison of the Pre-Reacting Complexes (PRC) of $S$ and $R$ enantiomers by Activation Strain analysis in the stepwise pathway}

Table S2. Computed Relative Distortion Energies $(\mathrm{kcal} / \mathrm{mol})$ in each Fragment, Total Relative Distortion Energies $\left(\Delta \mathrm{E}_{\mathrm{d}}^{\ddagger}\right)$ and Relative Interaction Energies $\left(\Delta \mathrm{E}_{\mathrm{i}}^{\dagger}\right)$ of Pre-reacting complex of the Stereocontrolling Transition States $[6-7]_{S_{-} \text {si_syn }}{ }^{*}$ and $[6-7]_{R_{-} s i}$ antit ${ }^{*}$ with respect to unstrained substrate geometries at the M06-2X/6-31G** Level of Theory for both the enantiomers in the stepwise pathway ${ }^{\mathrm{a}}$

\begin{tabular}{|c|c|c|c|c|c|}
\hline TS & $\Delta E_{\mathrm{d}}^{\dagger}(\mathrm{f} 1)$ & $\Delta E_{\mathrm{d}}^{\dagger}(\mathrm{f} 2)$ & $\begin{array}{c}\Delta E_{\mathrm{d}}^{\ddagger}(\text { total })= \\
\Delta E_{\mathrm{d}}^{*}(\mathrm{f} 1)+\Delta E_{\mathrm{d}}^{+}(\mathrm{f} 2)\end{array}$ & $\Delta E_{\mathrm{i}}^{\dagger}$ & $\begin{array}{c}\Delta E^{\dagger}= \\
{\left[\Delta E^{+}{ }_{\mathrm{d}}^{+}+\Delta E_{\mathrm{i}}^{+}\right]}\end{array}$ \\
\hline$[6-7]_{S \text { si syn }}{ }^{*}$ & 4.7 & 2.9 & 7.6 & -35.9 & -28.2 \\
\hline$[6-7]_{R \text { si anti }}$ & 7.0 & 4.1 & 11.2 & -39.9 & -28.7 \\
\hline
\end{tabular}

${ }^{\mathrm{a}} \mathrm{f} 1$ and $\mathrm{f} 2$ are respectively the catalyst, BINOL phosphoric acid and the substrate $\alpha$-methyl $\gamma$-hydroxy ester

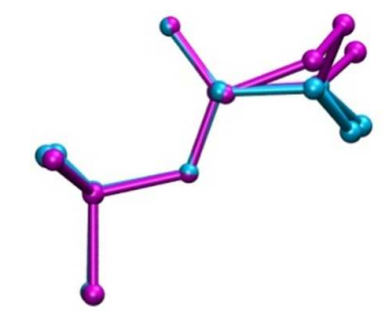

$\alpha$-methyl $\gamma$-hydroxy ester



$\alpha$-methyl $\gamma$-hydroxy ester 



Figure S1. Overlaid Images of both substrate and catalyst as seen in the initial recognition complex of stereocontrolling transition states $[6-7]_{S_{-} s i \_s y n}{ }^{*}$ and $[6-7]_{R_{-} s i \_a n t i}{ }^{*}$ for both the $S$ and $R$ enantiomers of the substrate. Cyan color (lower energy) and Magenta color (higher energy). For clarity hydrogen atoms are removed.

The substrate in the PRC of $[6-7]_{R_{-} s i}$ anti ${ }^{*}$ is deformed by $1.24 \mathrm{kcal} / \mathrm{mol}$ as compared to the PRC of [6-7 $]_{S_{-} \text {sissyn }}{ }^{*}$ whereas the deformation in the catalyst is $2.25 \mathrm{kcal} / \mathrm{mol}$. PRC of [6$7]_{R_{-} \text {si_anti }}{ }^{*}$ exhibits $4.06 \mathrm{kcal} / \mathrm{mol}$ more attractive interaction than in the PRC of [6-7] $]_{S_{-} s i s y n}{ }^{*}$. So the net balance between the destabilizing distortion and stabilizing interaction energies


stability of PRC of $[6-7]_{R_{-} s i}$ anti ${ }^{*}$ results from the higher interaction energy between the reacting partners. It is interesting to note that the elementary step barrier with respect to the corresponding PRC is $2 \mathrm{kcal} / \mathrm{mol}$ lower for $[6-7]_{S_{-} s i_{-} s y n}{ }^{\star}$ than the diastereomeric $[6-7]_{R_{-} s i_{-} a n t i}{ }^{*}$.

\section{Concerted Pathway}

In the concerted pathway, the BINOL-phosphoric acid protonates the oxygen of the tertbutoxy group and the lactonization is found to proceed in a concerted manner. In this pathway, the protonation is accompanied by an intramolecular attack of the hydroxy group to the carbonyl carbon, resulting in the expulsion of tert-butanol. In other words, the ring closing $\mathrm{C}-\mathrm{O}$ bond formation and the cleavage of $\mathrm{C}-\mathrm{O}^{\mathrm{t}} \mathrm{Bu}$ bond resulting in the removal of tert-butanol are concerted. The catalyst plays a bifunctional role as perceptible from $[3-4]^{*}$, 
wherein it recovers a proton from the hydroxyl end of the substrate while donating its proton to the other end to the departing tert-butoxyl group.

(a)



(b)



Figure S2. (a) Atom numbering scheme used in the Intrinsic Reaction Coordinate (IRC) calculations, (b) The IRC plots for the lowest energy transition state $[3-4]_{S_{-} \text {si_anti }}$ in the concerted pathway. The X-axis corresponds to number of IRC steps (reaction coordinate) starting from the transition state and the Y-axis corresponds to the energies at each of the IRC 
points. The important distances b1, b2, b3, b4, b5, and b6, as shown in Figure S2, are provided at each point along the potential energy profile in the order. The reaction profile along the intrinsic reaction coordinate (IRC) trajectory calculated at the M06-2X/6-31G**for the $\mathrm{C}-\mathrm{O}$ bond formation, clearly shows that the ring closing $\mathrm{C}-\mathrm{O}$ bond formation, as indicated by $\mathrm{b} 1$, and the cleavage of $\mathrm{C}-\mathrm{O}^{\mathrm{t}} \mathrm{Bu}$ bond resulting in the removal of tert-butanol (b2) take place in a concerted manner. It can be noticed in the forward direction from the transition state toward product side, b1 decreases and while b2 undergoes a gradual increase. Toward the reactant side, b1 increases when b2 shows a steady decrease.

\section{Conformational Analysis}

Depending upon (a) the attack of the nucleophilic hydroxy group to the carbonyl carbon, either through re or si prochiral face, and (b) the relative orientation of the $\beta$-methylene with respect to the ester carbonyl group, we have considered the following key conformers of the substrate in the concerted pathway.


$$
\text { R_si_syn }
$$

$$
\text { R_si_anti }
$$

R_re_anti 
Figure S3. Different possible conformers and configurations of diastereomeric transition states for the stereocontrolling $\mathrm{C}-\mathrm{O}$ bond formation via $[3-4]^{*}$ for the concerted pathway. ${ }^{a}$

${ }^{a}$ For catalyst the orientation of the isopropyl group measured as $\Phi_{1}(\mathrm{H} 9-\mathrm{C} 10-\mathrm{C} 11-\mathrm{C} 12)$ and $\Phi_{2}(\mathrm{H} 13-\mathrm{C} 14-\mathrm{C} 15-\mathrm{C} 16)$ dihedral angle as shown in Figure 1 in the manuscript can either be $0^{\circ}$ or $180^{\circ}$. The position of other isopropyl groups on phenyl rings is kept constant since the variation of dihedral angle of those does not make any such impact on stereoselectivity being not involved in any type of noncovalent interactions.

Table S3. The Relative Gibbs Free Energies (kcal/mol) of the Different Diastereomeric Transition States $[3-4]^{\star}$ for the C-O Bond Formation with Respect to the Lowest Energy Transition State for the Concerted Pathway

\begin{tabular}{|c|c|c|}
\hline [3-4] $^{*}$ & L1 & L2 \\
\hline S_si_syn & 2.6 & 3.2 \\
\hline S_re_syn & 12.1 & 10.9 \\
\hline R_si_syn & 5.0 & 5.4 \\
\hline R_re_syn & 9.5 & 9.0 \\
\hline S_si_anti & 0.0 & 0.0 \\
\hline S_re_anti & 1.7 & 1.6 \\
\hline R_si_anti & 0.9 & 0.9 \\
\hline R_re_anti & 9.2 & 7.0 \\
\hline
\end{tabular}

$$
[3-4]_{\text {S_si_anti }} \ddagger
$$

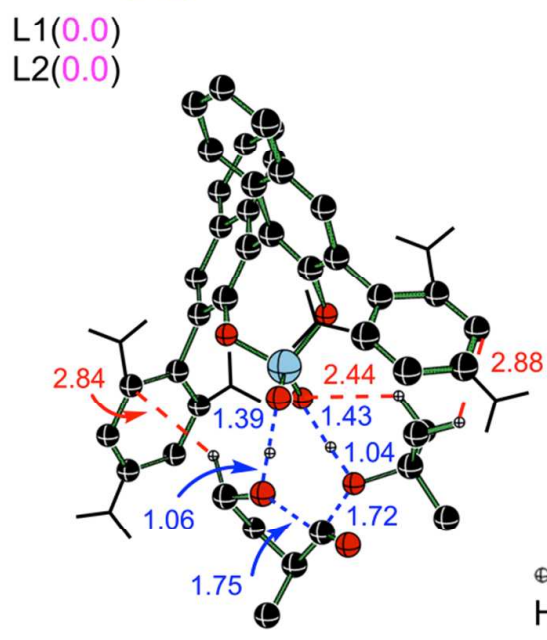



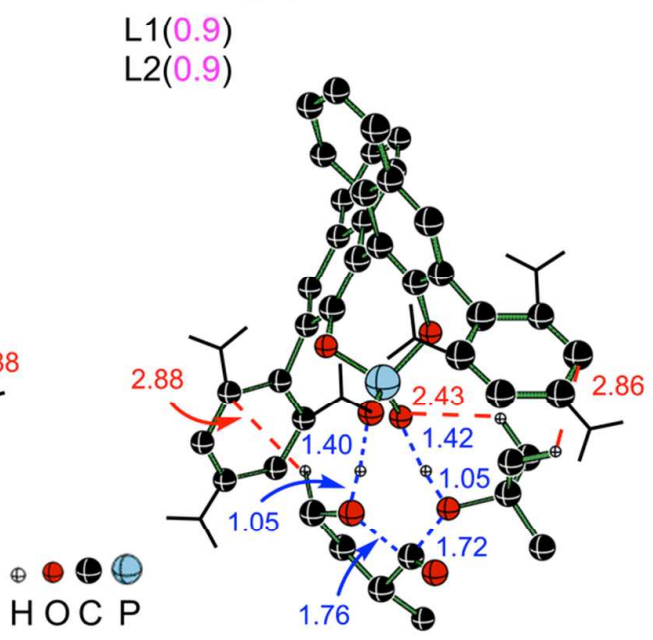

Figure S4. The optimized geometries of the lower energy diastereomeric transition states [3$4]^{*}$ at the M06-2X/6-31G** level of theory for the stereoselective $\mathrm{C}-\mathrm{O}$ bond formation in the 
concerted pathway. L1 = M06-2X/6-31G**, L2 $=\operatorname{SMD}_{(\mathrm{DCM})} / \mathrm{M} 06-2 \mathrm{X} / 6-31 \mathrm{G}^{* *} / / \mathrm{M} 06-2 \mathrm{X} / 6-$ $31 G^{* *}$. Only select hydrogens are shown for improved clarity. The bond lengths are in $\AA$.

Table S4. The Relative Gibbs Free Energies (kcal/mol) of the Different Diastereomeric Transition States $[6-7]^{*}$ for the $\mathrm{C}-\mathrm{O}$ Bond Formation with Respect to the Lowest Energy Transition State via Stepwise Pathway

\begin{tabular}{|c|c|c|c|c|}
\hline \multicolumn{2}{|c|}{ Transition states } & $\begin{array}{l}\text { Dihedral angle } \\
\text { H1-C2-C3-C4 }\end{array}$ & L1 & L2 \\
\hline \multirow{4}{*}{ S_re_syn } & i & $-168.0^{\circ}, 50.7^{\circ}$ & 5.4 & 5.2 \\
\hline & ii & $-169.8^{\circ}, 148.2^{\circ}$ & 5.1 & 5.1 \\
\hline & ii & $-49.9^{\circ}, 145.9^{\circ}$ & 6.4 & 6.1 \\
\hline & iv & $-48.3^{\circ}, 51.3^{\circ}$ & 4.2 & 4.4 \\
\hline \multirow{4}{*}{ S_si_syn } & $\mathbf{i}$ & $-159.7^{0},-44.4^{o a}$ & 5.1 & 4.5 \\
\hline & ii & $-11.2^{\circ},-172.2^{\circ}$ & 1.1 & 0.9 \\
\hline & ii & $-12.0^{\circ}, 17.2^{\circ}$ & 0.0 & 0.0 \\
\hline & iv & $-121.2^{\circ}, 141.0^{\circ}$ & 2.0 & 1.6 \\
\hline \multirow{4}{*}{ R_re_syn } & $\mathbf{i}$ & $-49.2^{\circ}, 142.1^{\circ}$ & 3.8 & 3.8 \\
\hline & ii & $-157.6^{\circ}, 3.8^{\circ}$ & 4.1 & 3.9 \\
\hline & ii & $0.2^{\circ}, 4.3^{\circ}$ & 5.4 & 5.2 \\
\hline & iv & $166.6^{\circ}, 147.9^{\circ}$ & 4.1 & 4.1 \\
\hline \multirow{4}{*}{ R_si_syn } & i & $-158.7^{\circ},-4.4^{\circ}$ & 4.4 & 4.3 \\
\hline & ii & $-157.8^{\circ},-179.0^{\circ}$ & 3.5 & 3.5 \\
\hline & ii & $-10.9^{\circ}, 24.8^{\circ}$ & 1.6 & 1.7 \\
\hline & iv & $-10.0^{\circ}, 141.0^{\circ}$ & 4.7 & 4.6 \\
\hline \multirow{4}{*}{ S_re_anti } & i & $-133.4^{\circ}, 4.2^{\circ}$ & 1.8 & 1.5 \\
\hline & ii & $-42.8^{\circ}, 128.8^{\circ}$ & 2.1 & 1.6 \\
\hline & ii & $-133.1^{\circ}, 131.7^{\circ}$ & 1.6 & 1.1 \\
\hline & iv & $51.8^{\circ},-1.9^{\circ}$ & 2.3 & 1.7 \\
\hline \multirow{4}{*}{ S_si_anti } & $\mathbf{i}$ & $-149.5^{\circ}, 137.9^{\circ}$ & 2.5 & 1.9 \\
\hline & ii & $-1.4^{\mathrm{o}}, 38.1^{\mathrm{o}}$ & 5.3 & 4.3 \\
\hline & ii & $-148.8^{\circ}, 41.4^{\circ}$ & 4.4 & 3.5 \\
\hline & iv & $-0.6^{\circ}, 139.4^{\circ}$ & 2.7 & 2.0 \\
\hline \multirow{4}{*}{ R_re_anti } & i & $175.6^{\circ}, 133.4^{o b}$ & 5.6 & 4.2 \\
\hline & ii & $-40.6^{\circ}, 137.8^{\circ}$ & 4.8 & 3.4 \\
\hline & ii & $-4.4^{\circ}, 16.8^{\circ}$ & 3.1 & 1.8 \\
\hline & iv & $163.8^{\circ},-7.0^{\circ}$ & 3.7 & 2.2 \\
\hline \multirow{3}{*}{ R_si_anti } & $\mathbf{i}$ & $45.7^{\circ},-176.6^{o c}$ & 3.4 & 2.8 \\
\hline & ii & $45.1^{\circ}, 3.2^{\circ d}$ & 2.3 & 1.2 \\
\hline & ii & $-165.2^{\circ},-178.8^{\circ}$ & 3.0 & 2.3 \\
\hline
\end{tabular}




\begin{tabular}{|c|c|c|c|c|}
\hline & iv & $-168.7^{\circ}, 3.53^{\circ}$ & 1.5 & 0.9 \\
\hline
\end{tabular}

$\mathrm{LI}=\mathrm{M} 06-2 \mathrm{X} / 6-31 \mathrm{G}^{* *}, \mathrm{~L} 2=\mathrm{SMD}_{(\mathrm{DCM})} / \mathrm{M} 06-2 \mathrm{X} / 6-31 \mathrm{G}^{* *} / / \mathrm{M} 06-2 \mathrm{X} / 6-31 \mathrm{G}^{* *}$. An additional imaginary frequency of (a) $-12.49(b)-8.05(c)-1.99(d)-11.69$ is observed in the optimized geometry of transition state $S \_s i \_s y n$ (i), $R \_r e \_a n t i(i), R \_s i \_a n t i(i), R \_s i \_a n t i(i i)$ respectively.

$V$. Wiberg bond indices of selected bonds in the stereocontrolling $[6-7]_{S_{-} s i s y n}{ }^{*}$ in the

\section{stepwise pathway}


Figure S5. Plot of Wiberg Bond Indices of selected bonds, $\mathrm{C} 1-\mathrm{O} 2$ and $\mathrm{O} 3-\mathrm{H} 4$ for the stereocontrolling lowest energy transition state $[6-7]_{S_{-} s i s y n}{ }^{*}$ in the stepwise pathway. The Xaxis corresponds to reaction coordinate and the $\mathrm{Y}$-axis is the Wiberg Bond Indices at specified points. It can be noticed from the Wiberg Bond Indices that both the bonds $\mathrm{C} 1-\mathrm{O} 2$ and $\mathrm{O} 3-\mathrm{H} 4$ exhibits a progressive increase indicating both the bonds are being formed as the reaction proceeds. 


\section{Gibbs free energy profile}

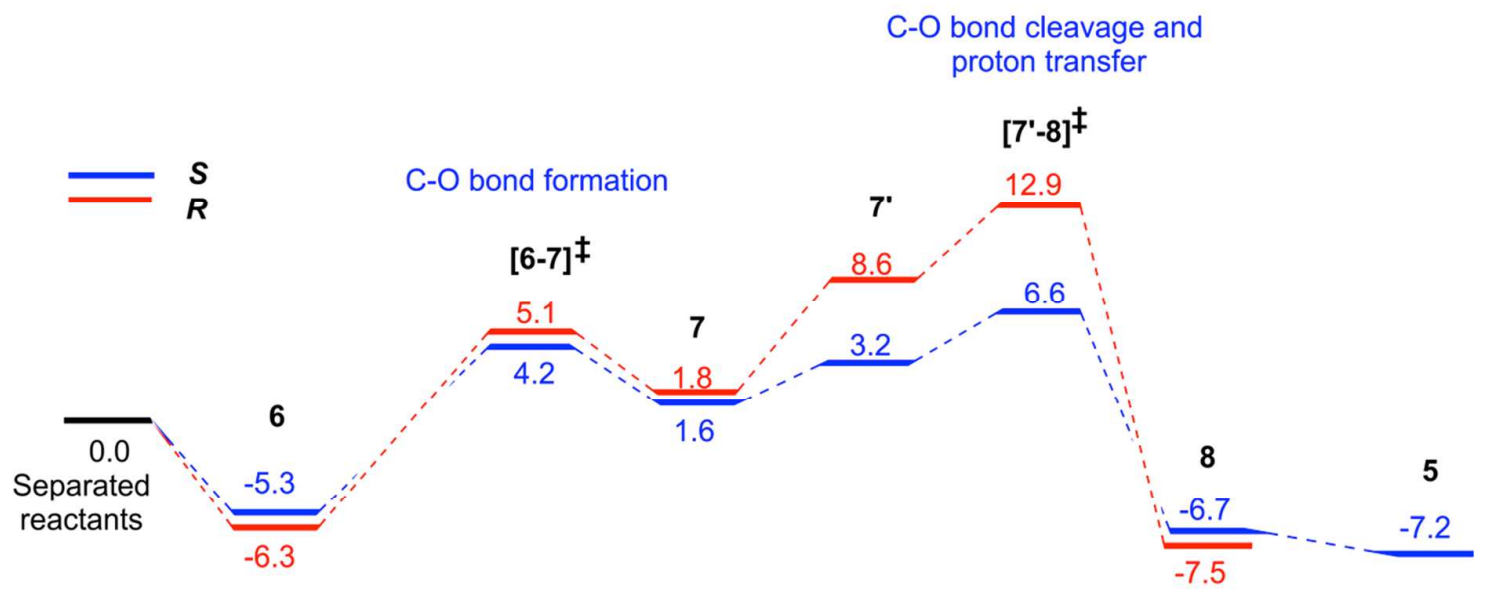

Figure S6. Gibbs free energy profile at the $\operatorname{SMD}_{(\mathrm{DCM})} / \mathrm{M} 06-2 \mathrm{X} / 6-31 \mathrm{G}^{* *} / \mathrm{M} 06-2 \mathrm{X} / 6-31 \mathrm{G}^{* *}$ level of theory. All the energies are in $\mathrm{kcal} / \mathrm{mol}$. It can be noticed that the activation barrier for the stereocontrolling $\mathrm{C}-\mathrm{O}$ bond formation step, calculated as the energy difference between $[6-7]^{\ddagger}$ and 6 is about $9.5 \mathrm{kcal} / \mathrm{mol}$ for the ring closing transition state for the $S$ enantiomer, which is $1.9 \mathrm{kcal} / \mathrm{mol}$ lower than that for the $R$ enantiomer. The transition state for the $\mathrm{C}-\mathrm{O}$ bond cleavage and proton transfer $\left[\mathbf{7}^{\prime} \mathbf{- 8}\right]^{\ddagger}$ exhibits the highest barrier of 11.9 $\mathrm{kcal} / \mathrm{mol}$ for the $S$ enantiomer against the $19.2 \mathrm{kcal} / \mathrm{mol}$ for the $R$ enantiomer (calculated as the energy difference between $\left[7^{\prime}-\mathbf{8}\right]^{\ddagger}$ and $\mathbf{6}$.

\section{Calculation of enantiomeric excess (ee) of unreacted hydroxy ester (1a)}



Racemic mixture of $\alpha$-methyl $\gamma$-hydroxy ester contains equal amounts of $S$ and $R$ enantiomers. Suppose ' $a_{0}$ ' is the initial totoal number of moles of the racemic mixture. During lactonization, 'x' moles of $S$ enantiomer gets converted to $S$ lactone and 'y' moles of 
$R$ enantiomer goes to $R$ lactone. The experimental total conversion is $42 \%$ and the computationally computed enantiomeric excess of $S$ lactone (2a) is $64 \%$. The enantiomeric excess of recovered hydroxy ester (1a) can be calculated using the following formulation, Enantiomeric excess (ee) of $S$ lactone (2a)

$\frac{\mathrm{x}-\mathrm{y}}{x+y}=e e$ Total Conversion $=42 \%$ (expt.)

$x-y=0.64 *\left(0.42 \mathrm{a}_{\mathrm{o}}\right)$

Therefore, $\mathrm{x}+\mathrm{y}=0.42 \mathrm{a}_{\mathrm{o}}$

Enantiomeric excess (ee) of the recovered hydroxy ester (1a)

Since racemic mixture contains equal amounts of both the enantiomers, $S: R=50: 50$. So, ' $0.5 \mathrm{a}_{\mathrm{o}}-\mathrm{x}$ ' moles of unreacted $S$ enantiomer and ' $0.5 \mathrm{a}_{\mathrm{o}}-\mathrm{y}$ ' moles of unreacted $R$ enantiomer gives the enantiomeric excess of the unreacted hydroxy ester as:

$\frac{\left(0.5 a_{0}-y\right)-\left(0.5 a_{0}-x\right)}{\left(0.5 a_{0}-y\right)+\left(0.5 a_{0}-x\right)}=e e$

$\frac{x-y}{\mathrm{a}_{\mathrm{o}}-(\mathrm{x}+\mathrm{y})}=e e$

Substituting equations (1) and (2) in equation (4), the enantiomeric excess (ee) of recovered hydroxy ester is calculated as $46.3 \%$.

VIII. Details of Activation Strain analysis on the Stereocontrolling Transition States in the stepwise pathway

Table S5. Computed Relative Distortion Energies $(\mathrm{kcal} / \mathrm{mol})$ in each Fragment, Total Relative Distortion Energies $\left(\Delta \mathrm{E}_{\mathrm{d}}^{\ddagger}\right)$ and Relative Interaction Energies $\left(\Delta \mathrm{E}_{\mathrm{i}}^{+}\right)$of the

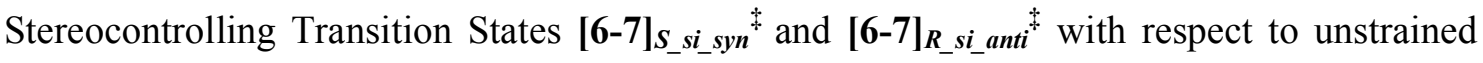
substrate geometries at the M06-2X/6-31G** Level of Theory for both the enantiomers in the stepwise pathway $^{\mathrm{a}}$

\begin{tabular}{|c|c|c|c|c|c|}
\hline $\mathrm{TS}$ & $\Delta E_{\mathrm{d}}^{\ddagger}(\mathrm{f} 1)$ & $\Delta E_{\mathrm{d}}^{\ddagger}(\mathrm{f} 2)$ & $\begin{array}{c}\Delta E^{\ddagger} \text { (total)= } \\
\Delta E_{\mathrm{d}}^{\ddagger}(\mathrm{f} 1)+\Delta E_{\mathrm{d}}^{\ddagger}(\mathrm{f} 2)\end{array}$ & $\Delta E_{\mathrm{i}}^{\ddagger}$ & $\begin{array}{c}\Delta E^{\ddagger}= \\
{\left[\Delta E_{\mathrm{d}^{+}}^{\ddagger} \Delta E_{\mathrm{i}}^{\ddagger}\right.}\end{array}$ \\
\hline$[6-7]_{S \text { si syn }}^{\ddagger}$ & 63.7 & 37.8 & 101.5 & -121.0 & -19.5 \\
\hline$[6-7]_{R \text { si anti }}^{\ddagger}$ & 66.7 & 45.3 & 112.0 & -131.0 & -19.0 \\
\hline
\end{tabular}


${ }^{a} \mathrm{f} 1$ and $\mathrm{f} 2$ are respectively BINOL phosphoric acid (catalyst) and the substrate $\alpha$-methyl $\gamma$-hydroxy ester

The substrate in the higher energy TS, namely $[6-7]_{R_{-} s i}$ anti ${ }^{*}$, is more deformed by 7.5 $\mathrm{kcal} / \mathrm{mol}$ as compared to that in the lowest energy $[6-7]_{S_{-} s i \leq s y n}{ }^{\ddagger}$. Similarly, the deformation in the catalyst part of the higher energy TS in $[6-7]_{R_{-} \text {si_anti }}$ is higher $3.0 \mathrm{kcal} / \mathrm{mol}$ over that in [6$7]_{S_{-} s i s y n}{ }^{*}$. On the other hand, the stabilizing interaction between the deformed catalyst and the substrate at the transition state geometry is $10.0 \mathrm{kcal} / \mathrm{mol}$ more in the higher energy [6$7]_{R_{-} s i \_a n t i}{ }^{*}$ that in the lower energy $[6-7]_{S_{-} s i_{-} s y n}{ }^{\ddagger}$. The overall balance between the distortion and interaction comes out to be in favour of TS of the lower energy $[6-7]_{S_{-} s i} i_{s y n}{ }^{*}$ by 0.5 $\mathrm{kcal} / \mathrm{mol}$.

\section{Topological Analysis of the Electron Density Using the AIM Formalism}

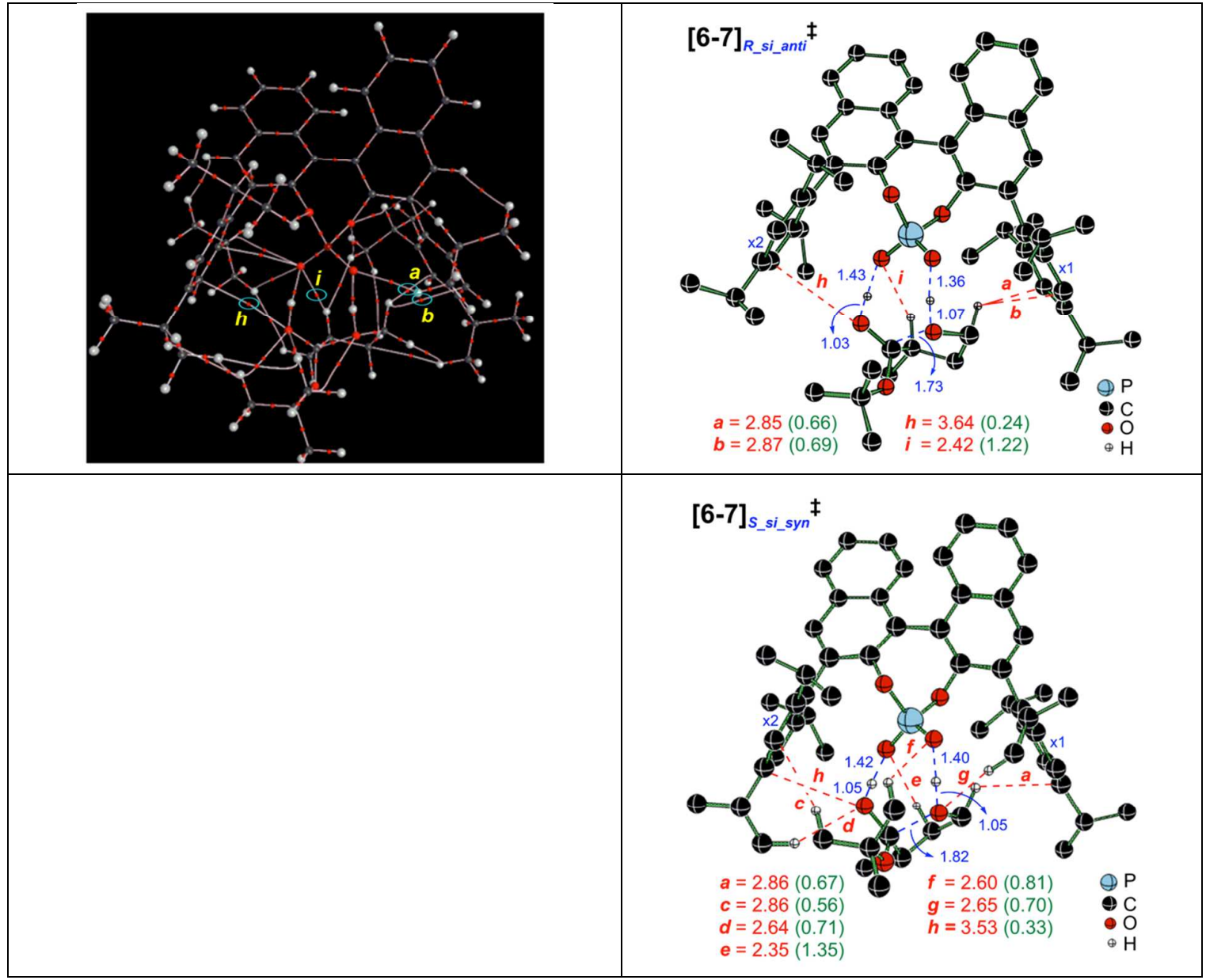


Figure S7. The bond critical points and the bond paths obtained from the topological analysis of the electron densities obtained using the Atoms-In-Molecule formalism on the stereocontrolling transition states $[6-7]_{R_{-} s i \_a n t i}{ }^{*}$ and $[6-7]_{S_{-} s i \_s y n}{ }^{\ddagger}$.

Table S6. Summary of Electron Densities $\left(\rho_{\mathrm{bcp}}\right)$ at the Bond Critical Points along the Bond

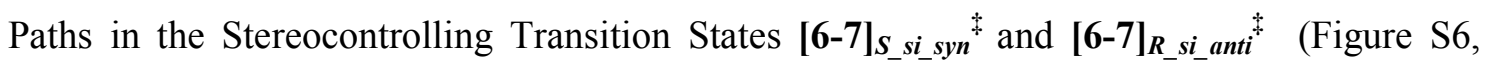
alphabetical labels used for the $b_{\mathrm{cps}}$ )

\begin{tabular}{|c|c|c|c|}
\hline \multirow{2}{*}{$\begin{array}{l}\text { Bond } \\
\text { path }\end{array}$} & \multirow{2}{*}{ Type of Interaction } & \multicolumn{2}{|c|}{$\rho_{\text {bcp }} * 10^{-2}$ (in a.u.) } \\
\hline & & {$[6-7]_{S \text { si sisyn }}$} & {$[6-7]_{R \_ \text {si_anti }}{ }^{*}$} \\
\hline $\mathrm{a}$ & Methylene $\mathrm{C}-\mathrm{H} \cdots \pi$ cloud of aryl & 0.6771 & $0.6669,0.6957$ \\
\hline $\mathrm{c}$ & $t$-butyl $\mathrm{C}-\mathrm{H} \cdots \pi$ cloud of aryl & 0.5618 & $-{ }^{a}$ \\
\hline $\mathrm{d}$ & Isopropyl $\mathrm{C}-\mathrm{H} \cdots \mathrm{O}$ of carbonyl & 0.7174 & $-{ }^{a}$ \\
\hline $\mathrm{e}$ & Methylene $\mathrm{C}-\mathrm{H} \cdots \mathrm{O}$ of binol & 1.3542 & $-{ }^{a}$ \\
\hline $\mathrm{f}$ & $t$-butyl $\mathrm{C}-\mathrm{H} \cdots \mathrm{O}$ of binol & 0.8110 & $-{ }^{a}$ \\
\hline $\mathrm{g}$ & Hydroxy $\mathrm{O} \cdots \mathrm{H}$ of isopropyl & 0.6997 & $-{ }^{a}$ \\
\hline $\mathrm{h}$ & Carbonyl $\mathrm{O} \cdots \pi$ cloud of aryl & 0.3324 & 0.2406 \\
\hline $\mathrm{i}$ & Methylene $\mathrm{C}-\mathrm{H} \cdots \mathrm{O}$ of binol & $-{ }^{a}$ & 1.2242 \\
\hline
\end{tabular}

${ }^{a-}$ No $b c p$ found<smiles>O=P(O)(Oc1cc2ccccc2cc1-c1cccc2ccccc12)Oc1c(-c2cc3ccccc3c3ccccc23)cc2ccccc2c1-c1cc2ccccc2c2ccccc12</smiles>

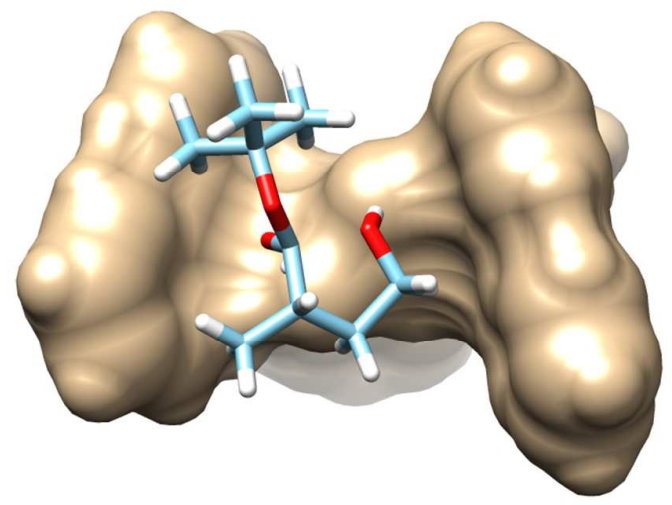

(a) BINOL Phosphoric acid with 9-phenylanthryl at 3,3' positions 
<smiles>[2H]c1c(C(F)(F)F)c(-c2cc3ccccc3cc2-c2c(OP(=O)(O)Oc3ccccc3)c(-c3cc(C(F)(F)F)c(C(F)(F)F)c(C(F)(F)F)c3C(F)(F)F)cc3ccccc23)cc(C(F)(F)F)c1C(F)(F)F</smiles>

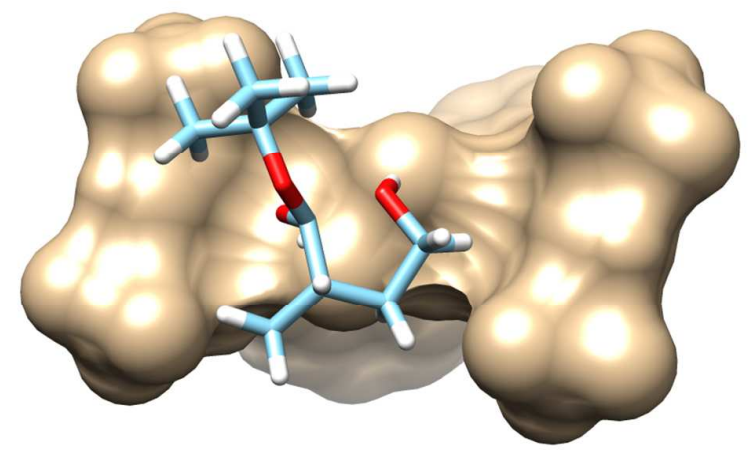

(b) BINOL Phosphoric acid with 3,5-( $\left(\mathrm{CF}_{3}\right)_{2} \mathrm{C}_{6} \mathrm{H}_{3}$ at 3,3' positions

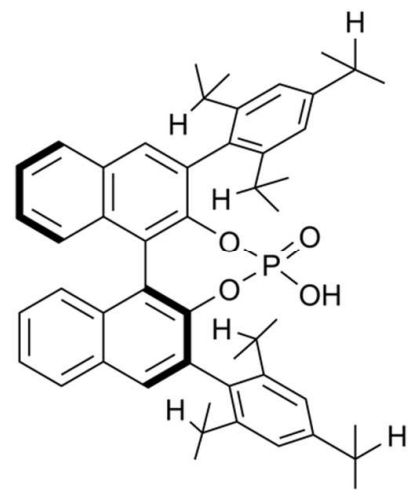

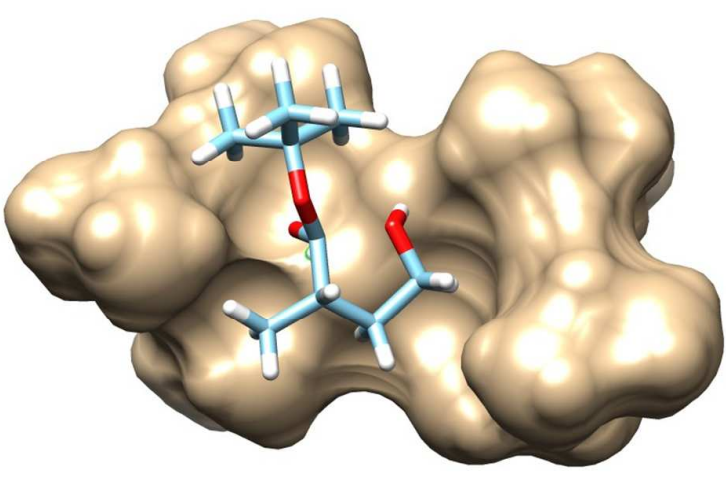

(c) BINOL Phosphoric acid with 2,4,6- $\left({ }^{i} \mathrm{Pr}\right){ }_{3} \mathrm{C}_{6} \mathrm{H}_{2}$ at 3,3' positions

Figure S8. Spacefilling models of the transition states for the $\mathrm{C}-\mathrm{O}$ bond formation in the case of (a) 9-phenylanthryl (b) 3,5-( $\left(\mathrm{CF}_{3}\right)_{2} \mathrm{C}_{6} \mathrm{H}_{3}$ and (c) 2,4,6- $\left({ }^{i} \mathrm{Pr}\right)_{3} \mathrm{C}_{6} \mathrm{H}_{2}$ substitution at the 3,3' positions of the BINOL backbone. In (a) and (b) the catalyst with 9-phenylanthryl and 3,5$\left(\mathrm{CF}_{3}\right)_{2} \mathrm{C}_{6} \mathrm{H}_{3}$ groups respectively at the $3,3^{\prime}$ positions provides a larger conformational space for the substrate thereby leading to relatively less effective non-covalent interactions. In (c), the catalyst provides a confined space wherein substrate appears to enjoy a better fit and improved non-covalent interactions.

(a)

(b)<smiles>CC(C)(C)OC(=O)[C@](C)(CCO)c1ccccc1</smiles><smiles>CC(C)(C)OC(=O)[C@@H](CCO)c1ccccc1</smiles> 

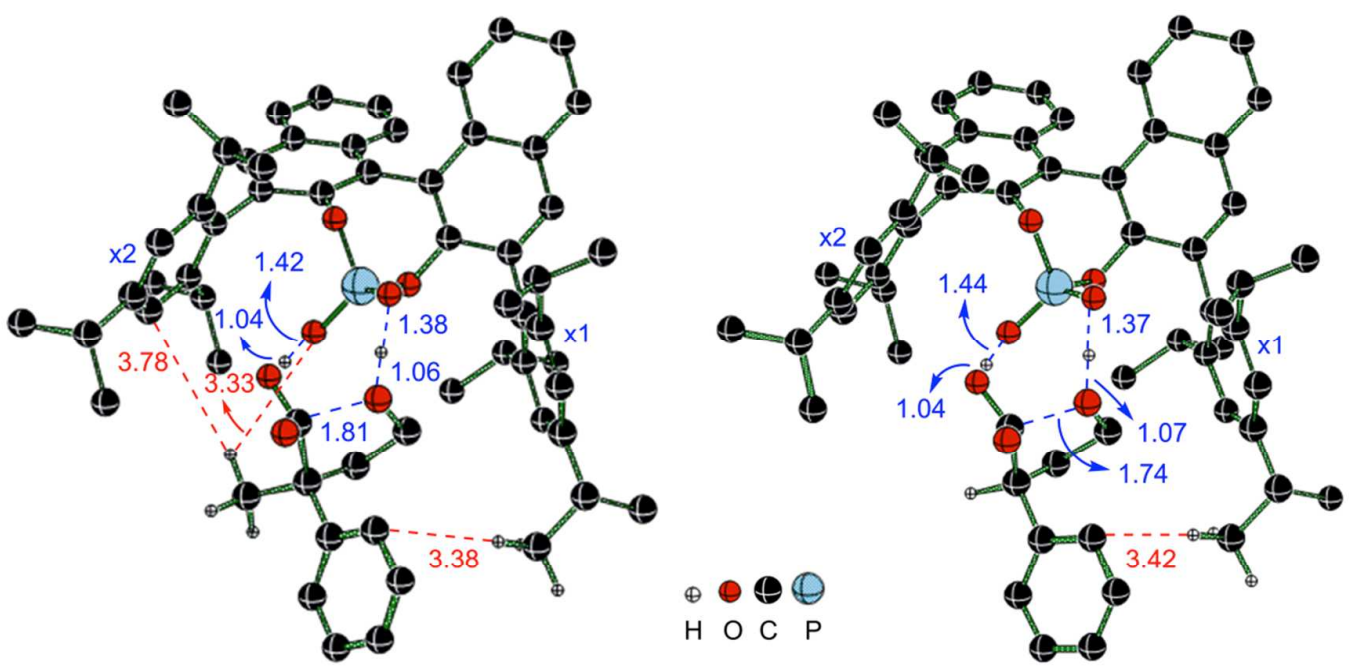

Figure S9. The optimized geometries of transition states (a) $\alpha$-methyl $\alpha$-phenyl $\gamma$-hydroxy ester (b) $\alpha$-phenyl $\gamma$-hydroxy ester with catalyst BINOL Phosphoric acid having 2,4,6$\left({ }^{i} \mathrm{Pr}\right)_{3} \mathrm{C}_{6} \mathrm{H}_{2}$ at $3,3^{\prime}$ positions at the M06-2X/6-31G** level of theory. Only selected hydrogens are shown for improved clarity. Inter-atomic contacts shown in red and blue color are in $\AA$. More number of favorable non-covalent interactions $(\mathrm{C}-\mathrm{H} \cdots \pi$ and $\mathrm{C}-\mathrm{H} \cdots \mathrm{O})$ are noticeable in (a) as compared to that in (b) due to the presence of both phenyl and methyl group at the chiral carbon atom of the substrate. In the latter only a phenyl group is present at the chiral carbon. Interestingly, higher enantioselectivity is reported for (a). 

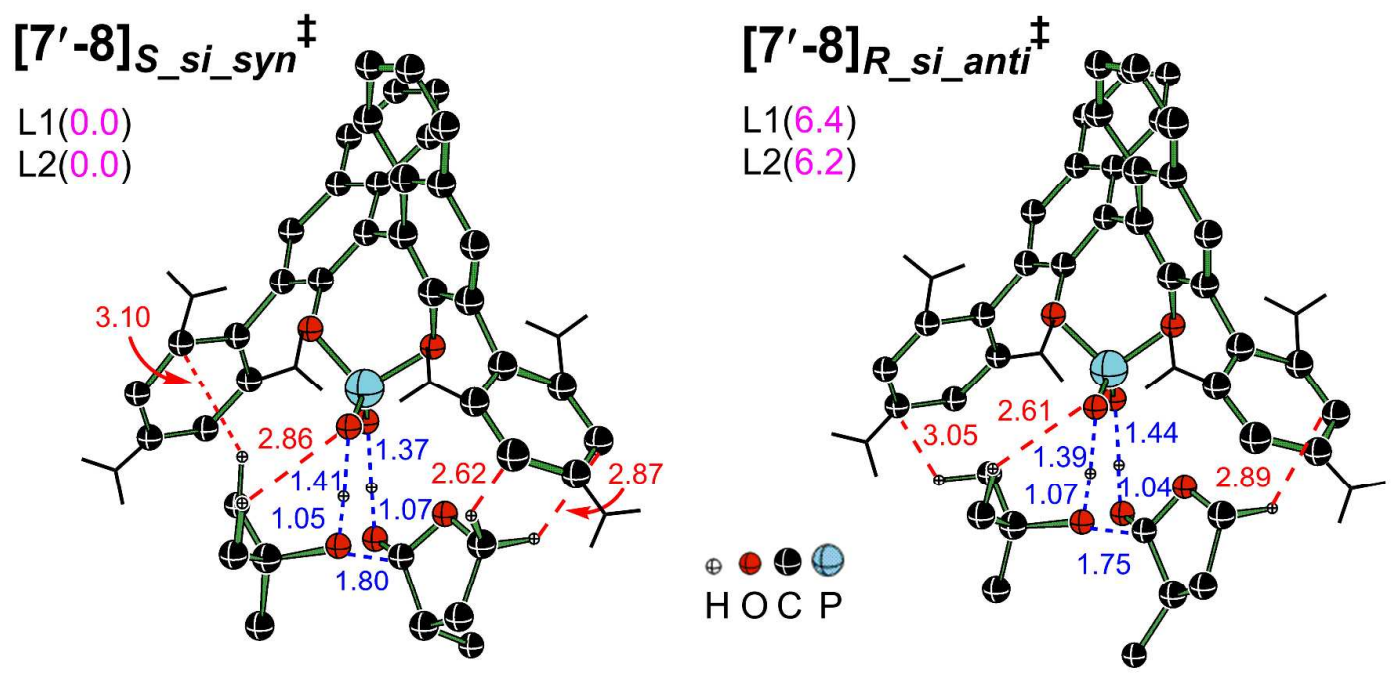

Figure S10. The optimized geometries of diastereomeric transition states at the M06-2X/6$31 \mathrm{G}^{* *}$ level of theory for the leaving of butyl group. L1 $=\mathrm{M} 06-2 \mathrm{X} / 6-31 \mathrm{G}^{* *}$, L2 $=$ $\mathrm{SMD}_{(\mathrm{DCM})} / \mathrm{M} 06-2 \mathrm{X} / 6-31 \mathrm{G}^{* *} / / \mathrm{M} 06-2 \mathrm{X} / 6-31 \mathrm{G}^{* *}$. Only selected hydrogens are shown for improved clarity. The bond lengths are in $\AA$. 


\section{The Total Energies, Number of Imaginary Frequencies and Optimized Cartesian}

\section{Coordinates of Reactants, Intermediates and the Transition States}

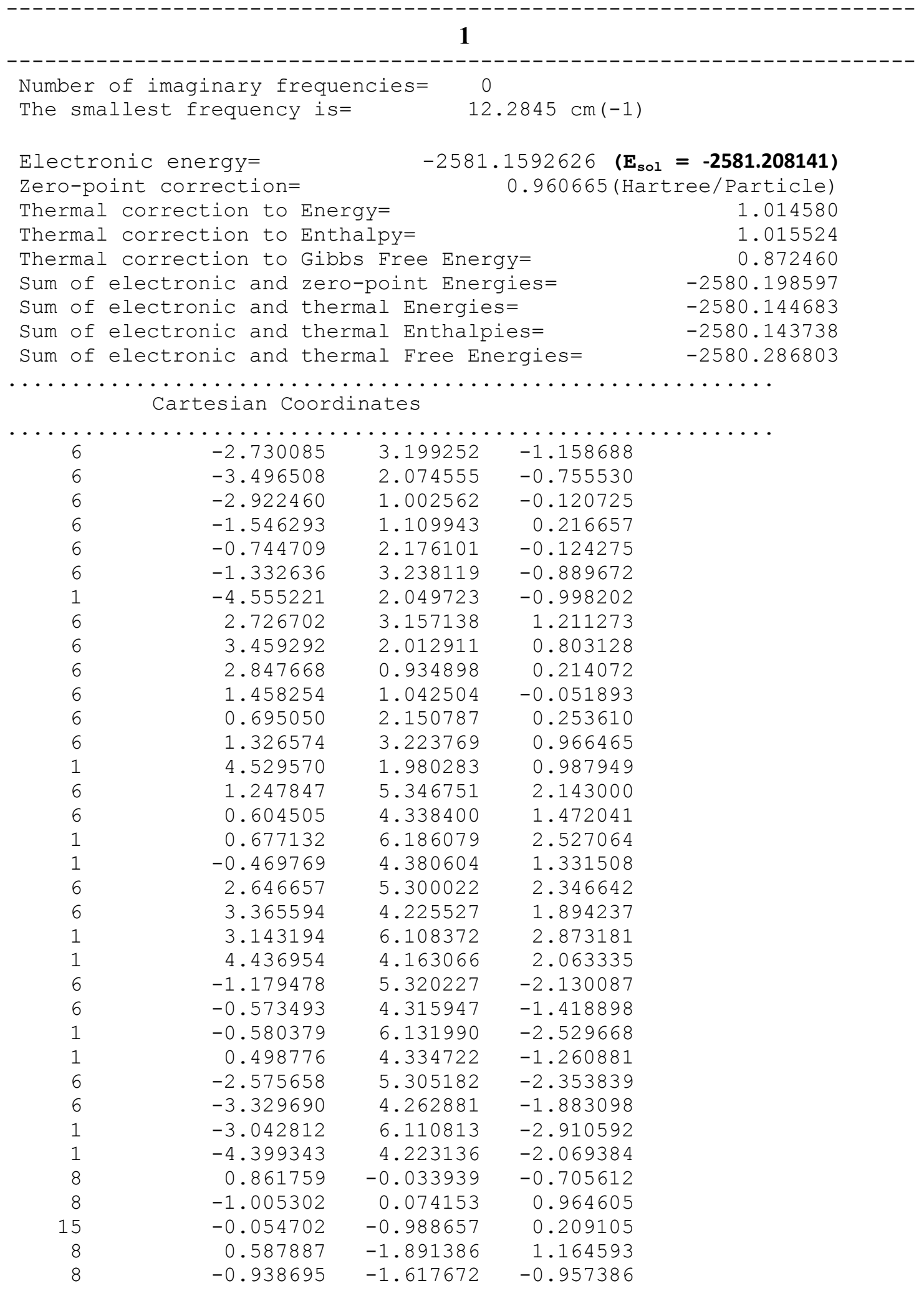




\begin{tabular}{|c|c|c|c|}
\hline 6 & 3.594130 & -0.310709 & -0.138845 \\
\hline 6 & 4.019424 & -1.183901 & 0.882950 \\
\hline 6 & 3.872706 & -0.601624 & -1.485134 \\
\hline 6 & 4.767562 & -2.306228 & 0.530378 \\
\hline 6 & 4.618449 & -1.742603 & -1.786225 \\
\hline 6 & 5.083378 & -2.600710 & -0.795347 \\
\hline 6 & -3.642964 & -0.279662 & 0.148694 \\
\hline 6 & -4.017795 & -1.109417 & -0.936725 \\
\hline 6 & -3.881269 & -0.698270 & 1.470267 \\
\hline 6 & -4.625874 & -2.335381 & -0.661472 \\
\hline 6 & -4.503665 & -1.929657 & 1.688536 \\
\hline 6 & -4.879139 & -2.764800 & 0.642542 \\
\hline 1 & -4.695257 & -2.250624 & 2.710185 \\
\hline 1 & -4.914033 & -2.975327 & -1.492102 \\
\hline 1 & 4.845594 & -1.969110 & -2.826347 \\
\hline 1 & 5.106844 & -2.977697 & 1.315717 \\
\hline 6 & -3.518200 & 0.154873 & 2.675270 \\
\hline 1 & -2.960338 & 1.028168 & 2.326301 \\
\hline 6 & -3.802364 & -0.708483 & -2.392088 \\
\hline 1 & -3.061815 & 0.096155 & -2.417654 \\
\hline 6 & -5.528790 & -4.108874 & 0.907140 \\
\hline 1 & -5.644939 & -4.204878 & 1.993871 \\
\hline 6 & -2.624370 & -0.601799 & 3.663823 \\
\hline 1 & -1.723258 & -0.978076 & 3.173440 \\
\hline 1 & -2.321097 & 0.062725 & 4.478330 \\
\hline 1 & -3.154945 & -1.448375 & 4.111803 \\
\hline 6 & -4.789314 & 0.669278 & 3.362828 \\
\hline 1 & -5.407580 & 1.245459 & 2.668494 \\
\hline 1 & -5.392961 & -0.162609 & 3.740690 \\
\hline 1 & -4.532238 & 1.310747 & 4.211088 \\
\hline 6 & -3.263994 & -1.845947 & -3.268382 \\
\hline 1 & -3.993674 & -2.653225 & -3.384390 \\
\hline 1 & -3.048627 & -1.461292 & -4.269422 \\
\hline 1 & -2.338280 & -2.265930 & -2.869115 \\
\hline 6 & -5.114358 & -0.181342 & -2.990683 \\
\hline 1 & -4.960717 & 0.153083 & -4.021138 \\
\hline 1 & -5.870453 & -0.974029 & -2.998418 \\
\hline 1 & -5.519386 & 0.654289 & -2.414436 \\
\hline 6 & -4.635942 & -5.257138 & 0.423185 \\
\hline 1 & -4.499635 & -5.210332 & -0.662459 \\
\hline 1 & -3.648041 & -5.212460 & 0.88932 \\
\hline 1 & -5.087597 & -6.224700 & 0.661587 \\
\hline 6 & -6.920857 & -4.196756 & 0.272595 \\
\hline 1 & -7.397863 & -5.148962 & 0.522902 \\
\hline 1 & -7.565263 & -3.384540 & 0.618748 \\
\hline 1 & -6.856350 & -4.133798 & -0.818666 \\
\hline 6 & 3.699183 & -0.939329 & 2.351192 \\
\hline 1 & 2.892709 & -0.200786 & 2.402447 \\
\hline 6 & 3.404099 & 0.289094 & -2.625007 \\
\hline 1 & 2.813185 & 1.108768 & -2.20495 \\
\hline 6 & 5.896236 & -3.831609 & -1.14811 \\
\hline 1 & 6.024936 & -3.835309 & -2.23801 \\
\hline 6 & 7.288253 & -3.785246 & -0.508928 \\
\hline 1 & 7.882829 & -4.651889 & -0.81389 \\
\hline 1 & 7.825061 & -2.877499 & -0.79679 \\
\hline 1 & 7.213546 & -3.799600 & 0.583348 \\
\hline 6 & 5.159711 & -5.116667 & -0.75473 \\
\hline 1 & 5.018182 & -5.165544 & 0.329 \\
\hline
\end{tabular}




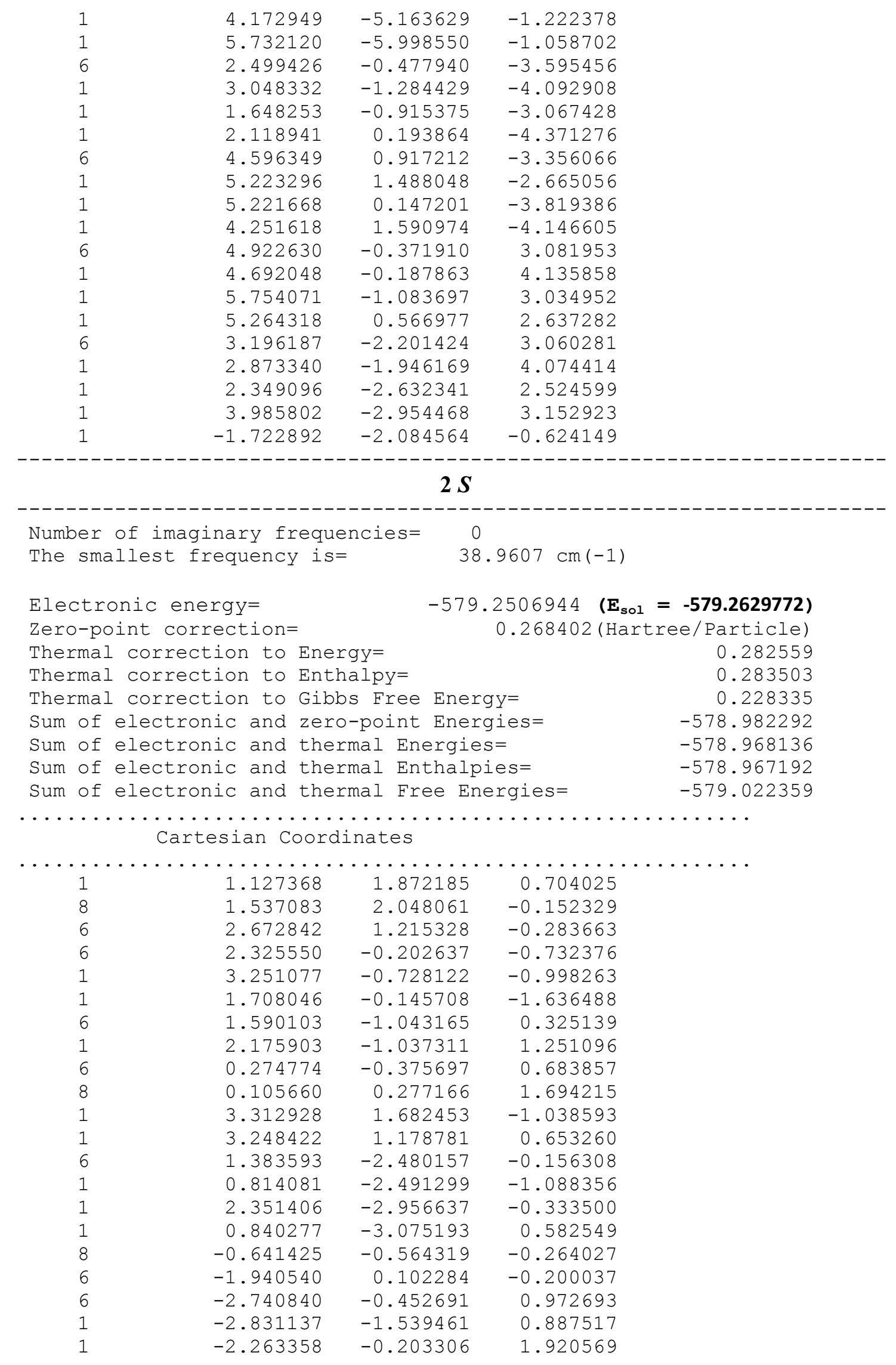




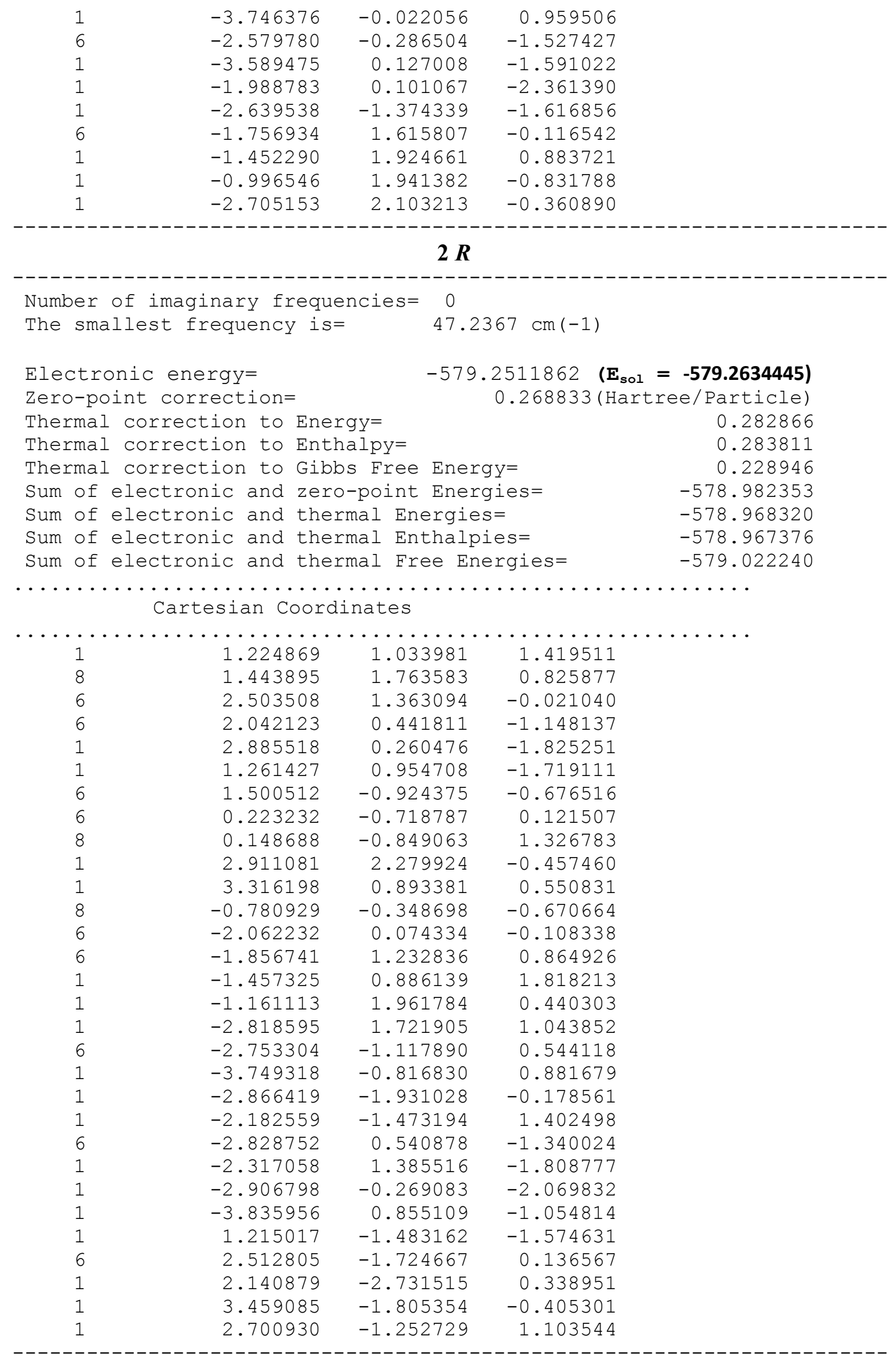




\section{$3 S$}

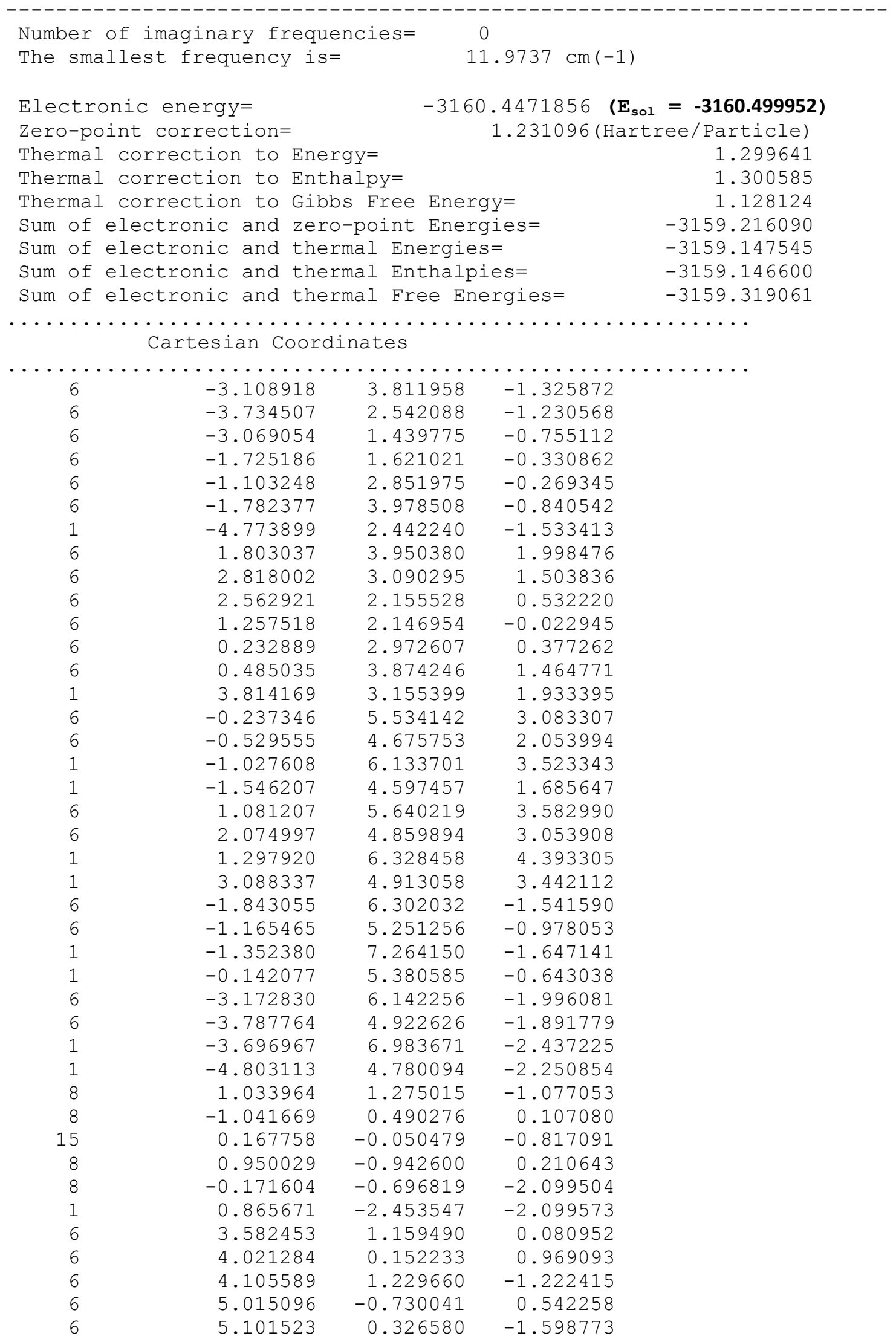




\begin{tabular}{|c|c|c|c|}
\hline 6 & 5.584303 & -0.646960 & -0.729799 \\
\hline 6 & -3.770162 & 0.130899 & -0.557845 \\
\hline 6 & -3.826961 & -0.824840 & -1.585656 \\
\hline 6 & -4.407261 & -0.108414 & 0.676148 \\
\hline 6 & -4.539652 & -2.005907 & -1.361668 \\
\hline 6 & -5.103827 & -1.303269 & 0.852078 \\
\hline 6 & -5.184895 & -2.263731 & -0.156037 \\
\hline 8 & 1.182651 & -2.977617 & -1.343668 \\
\hline 1 & -5.590954 & -1.492972 & 1.806064 \\
\hline 1 & -4.592022 & -2.751437 & -2.151096 \\
\hline 1 & 5.531898 & 0.398882 & -2.595668 \\
\hline 1 & 5.373425 & -1.490083 & 1.234252 \\
\hline 6 & -4.303770 & 0.870647 & 1.836792 \\
\hline 1 & -3.952652 & 1.830311 & 1.445354 \\
\hline 6 & -3.186677 & -0.571053 & -2.940063 \\
\hline 1 & -2.365673 & 0.137524 & -2.79703 \\
\hline 6 & -5.939711 & -3.560933 & 0.063795 \\
\hline 1 & -5.895589 & -4.126019 & -0.875564 \\
\hline 6 & -3.260773 & 0.368971 & 2.843630 \\
\hline 1 & -2.291471 & 0.214568 & 2.360172 \\
\hline 1 & -3.133875 & 1.083337 & 3.663357 \\
\hline 1 & -3.582367 & -0.588327 & 3.271280 \\
\hline 6 & -5.648944 & 1.125899 & 2.522841 \\
\hline 1 & -6.408308 & 1.442197 & 1.802387 \\
\hline 1 & -6.018995 & 0.231600 & 3.033653 \\
\hline 1 & -5.541626 & 1.910319 & 3.27783 \\
\hline 6 & -2.584158 & -1.834411 & -3.559651 \\
\hline 1 & -3.360820 & -2.515547 & -3.923850 \\
\hline 1 & -1.957714 & -1.561426 & -4.413298 \\
\hline 1 & -1.959490 & -2.363892 & -2.836631 \\
\hline 6 & -4.208455 & 0.059527 & -3.895599 \\
\hline 1 & -3.756019 & 0.243238 & -4.875114 \\
\hline 1 & -5.063678 & -0.611002 & -4.033668 \\
\hline 1 & -4.584054 & 1.010976 & -3.508454 \\
\hline 6 & -7.414360 & -3.305262 & 0.392986 \\
\hline 1 & -7.514334 & -2.765762 & 1.340518 \\
\hline 1 & -7.894960 & -2.707726 & -0.386140 \\
\hline 1 & -7.956912 & -4.250624 & 0.489334 \\
\hline 6 & -5.277096 & -4.409292 & 1.155347 \\
\hline 1 & -5.814901 & -5.352298 & 1.293786 \\
\hline 1 & -4.238565 & -4.637980 & 0.901412 \\
\hline 1 & -5.280321 & -3.877144 & 2.113401 \\
\hline 6 & 3.491556 & 0.030060 & 2.393057 \\
\hline 1 & 2.606308 & 0.666027 & 2.483361 \\
\hline 6 & 3.673031 & 2.288347 & -2.22562 \\
\hline 1 & 2.857316 & 2.870318 & -1.78888 \\
\hline 6 & 6.735990 & -1.545728 & -1.140336 \\
\hline 1 & 6.971990 & -1.312963 & -2.186262 \\
\hline 6 & 7.980616 & -1.244465 & -0.296794 \\
\hline 1 & 8.829594 & -1.851447 & -0.625639 \\
\hline 1 & 8.258286 & -0.189812 & -0.370017 \\
\hline 1 & 7.792966 & -1.469603 & 0.758313 \\
\hline 6 & 6.377519 & -3.031284 & -1.05911 \\
\hline 1 & 6.138029 & -3.316671 & -0.029067 \\
\hline 1 & 5.512809 & -3.265345 & -1.687772 \\
\hline 1 & 7.218082 & -3.649517 & -1.388407 \\
\hline 6 & 3.147407 & 1.658403 & -3.519676 \\
\hline 1 & 3.936162 & 1.103068 & -4.03783 \\
\hline
\end{tabular}









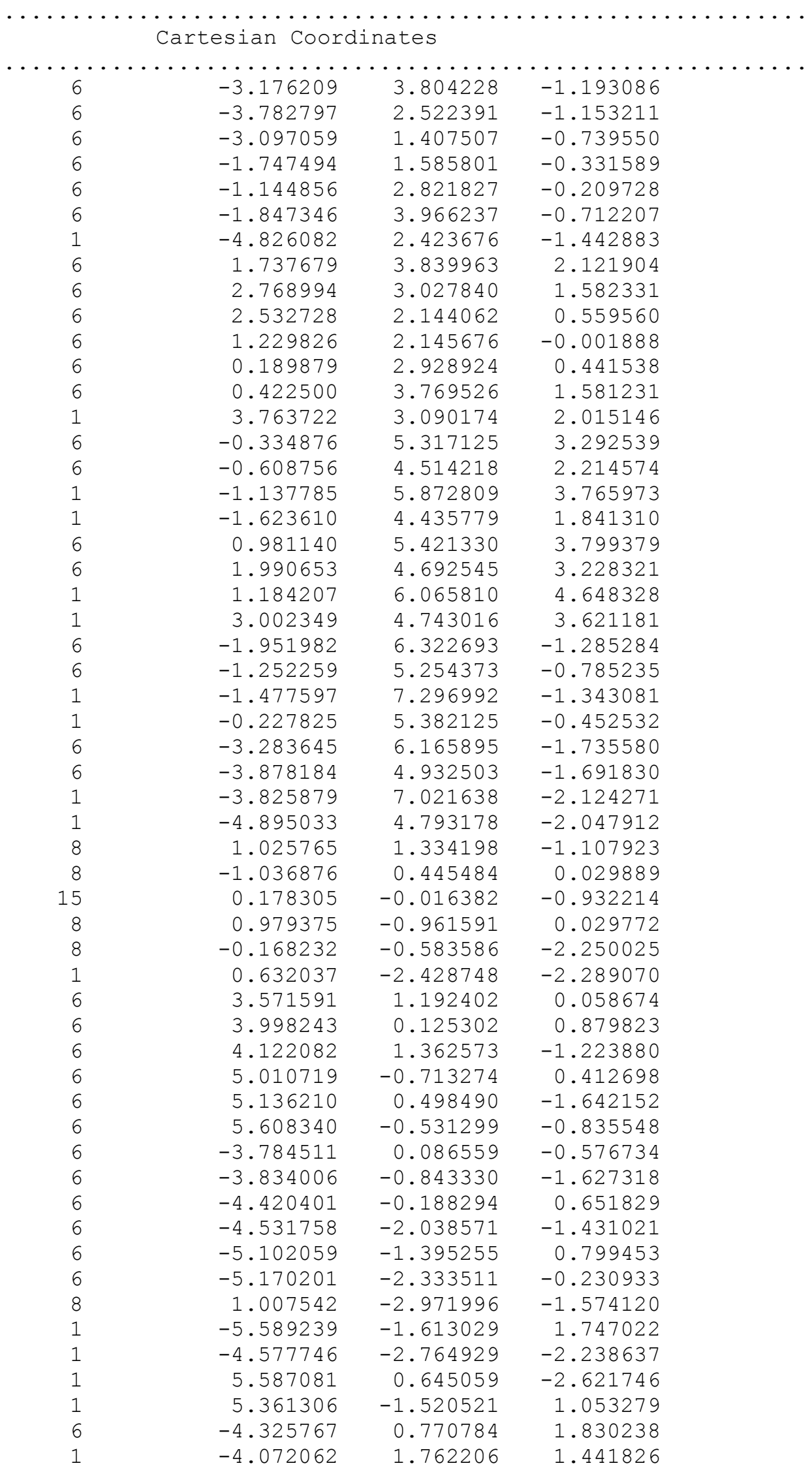




\begin{tabular}{|c|c|c|c|}
\hline 6 & -3.205587 & -0.547425 & -2.978180 \\
\hline 1 & -2.396358 & 0.172058 & -2.825345 \\
\hline 6 & -5.901552 & -3.648481 & -0.038206 \\
\hline 1 & -5.861492 & -4.185646 & -0.993991 \\
\hline 6 & -3.190650 & 0.333145 & 2.764986 \\
\hline 1 & -2.242404 & 0.245827 & 2.225989 \\
\hline ] & -3.063109 & 1.047223 & 3.584938 \\
\hline ] & -3.422431 & -0.647157 & 3.198346 \\
\hline 6 & -5.640395 & 0.909571 & 2.602552 \\
\hline 1 & -6.471580 & 1.153432 & 1.935314 \\
\hline 1 & -5.894490 & -0.009596 & 3.139502 \\
\hline 1 & -5.552092 & 1.704664 & 3.348671 \\
\hline 6 & -2.585527 & -1.786733 & -3.628476 \\
\hline 1 & -3.352234 & -2.469214 & -4.010579 \\
\hline 1 & -1.959638 & -1.485068 & -4.472699 \\
\hline 1 & -1.957576 & -2.327939 & -2.916389 \\
\hline 6 & -4.243599 & 0.087081 & -3.913322 \\
\hline 1 & -3.799914 & 0.301463 & -4.890625 \\
\hline 1 & -5.088435 & -0.594126 & -4.062418 \\
\hline 1 & -4.632289 & 1.022924 & -3.501588 \\
\hline 6 & -7.375055 & -3.428382 & 0.319641 \\
\hline 1 & -7.470809 & -2.919522 & 1.284364 \\
\hline 1 & -7.877265 & -2.816342 & -0.434190 \\
\hline 1 & -7.899827 & -4.385572 & 0.395206 \\
\hline 6 & -5.207564 & -4.515304 & 1.019002 \\
\hline 1 & -5.728761 & -5.469772 & 1.141318 \\
\hline 1 & -4.169854 & -4.721278 & 0.742556 \\
\hline 1 & -5.202574 & -4.008080 & 1.99045 \\
\hline 6 & 3.437863 & -0.104724 & 2.278779 \\
\hline 1 & 2.520532 & 0.483217 & 2.379751 \\
\hline 6 & 3.690056 & 2.481403 & -2.159164 \\
\hline 1 & 2.863486 & 3.024936 & -1.693662 \\
\hline 6 & 6.773127 & -1.394617 & -1.283083 \\
\hline 1 & 7.061071 & -1.055860 & -2.286279 \\
\hline 6 & 7.979577 & -1.204598 & -0.356470 \\
\hline 1 & 8.837789 & -1.781895 & -0.713739 \\
\hline 1 & 8.268565 & -0.152054 & -0.299068 \\
\hline 1 & 7.744521 & -1.544342 & 0.657680 \\
\hline 6 & 6.394912 & -2.874778 & -1.378279 \\
\hline 1 & 6.105350 & -3.265229 & -0.396468 \\
\hline 1 & 5.556127 & -3.022859 & -2.065177 \\
\hline 1 & 7.241618 & -3.468221 & -1.736570 \\
\hline 6 & 3.183441 & 1.930506 & -3.496472 \\
\hline 1 & 3.983579 & 1.417059 & -4.03997 \\
\hline 1 & 2.360985 & 1.227131 & -3.343208 \\
\hline 1 & 2.824373 & 2.747175 & -4.130097 \\
\hline 6 & 4.831413 & 3.483975 & -2.368771 \\
\hline 1 & 5.172660 & 3.894424 & -1.414034 \\
\hline 1 & 5.688421 & 3.007699 & -2.856045 \\
\hline 1 & 4.502232 & 4.312393 & -3.00329 \\
\hline 6 & 4.442077 & 0.380745 & 3.333924 \\
\hline 1 & 4.027972 & 0.269941 & 4.340693 \\
\hline 1 & 5.364265 & -0.208122 & 3.278749 \\
\hline 1 & 4.712206 & 1.429623 & 3.186467 \\
\hline 6 & 3.065167 & -1.565018 & 2.554602 \\
\hline 1 & 2.668484 & -1.655007 & 3.571237 \\
\hline 1 & 2.296137 & -1.921495 & 1.865850 \\
\hline 1 & 3.934830 & -2.228266 & 2.48846 \\
\hline
\end{tabular}









\begin{tabular}{|c|c|c|c|}
\hline 6 & 1.333163 & 1.869164 & 0.083541 \\
\hline 6 & 0.478669 & 2.915125 & 0.361019 \\
\hline 6 & 0.947297 & 3.955583 & 1.228956 \\
\hline 1 & 4.181513 & 2.857546 & 1.604436 \\
\hline 6 & 0.597659 & 5.978901 & 2.525141 \\
\hline 6 & 0.107303 & 5.001868 & 1.696482 \\
\hline 1 & -0.061807 & 6.765522 & 2.877131 \\
\hline 1 & -0.935628 & 5.014473 & 1.399528 \\
\hline 6 & 1.951974 & 5.966785 & 2.933004 \\
\hline 6 & 2.779916 & 4.957536 & 2.517200 \\
\hline 1 & 2.326679 & 6.748753 & 3.585197 \\
\hline 1 & 3.817315 & 4.922529 & 2.838394 \\
\hline 6 & -1.201807 & 6.109748 & -2.121848 \\
\hline 6 & -0.653423 & 5.100861 & -1.371401 \\
\hline 1 & -0.593024 & 6.963239 & -2.402104 \\
\hline 1 & 0.385506 & 5.155624 & -1.065207 \\
\hline 6 & -2.551100 & 6.045477 & -2.540874 \\
\hline 6 & -3.314338 & 4.955393 & -2.215676 \\
\hline 1 & -2.973160 & 6.853348 & -3.129603 \\
\hline 1 & -4.346244 & 4.881668 & -2.547702 \\
\hline 8 & 0.878193 & 0.828261 & -0.710543 \\
\hline 8 & -1.189460 & 0.721753 & 0.720854 \\
\hline 15 & -0.090264 & -0.244255 & 0.032908 \\
\hline 8 & 0.614324 & -0.984206 & 1.129210 \\
\hline 8 & -0.719671 & -1.060632 & -1.080277 \\
\hline 1 & -0.170149 & -2.217269 & -1.358826 \\
\hline 6 & 3.575954 & 0.676566 & 0.121291 \\
\hline 6 & 4.003132 & -0.278671 & 1.067393 \\
\hline 6 & 4.061462 & 0.630039 & -1.199193 \\
\hline 6 & 4.969697 & -1.214187 & 0.687266 \\
\hline 6 & 5.017890 & -0.331303 & -1.531336 \\
\hline 6 & 5.510547 & -1.241545 & -0.598377 \\
\hline 6 & -3.813952 & 0.426952 & -0.201996 \\
\hline 6 & -4.063556 & -0.510113 & -1.221195 \\
\hline 6 & -4.335694 & 0.224331 & 1.090573 \\
\hline 6 & -4.870819 & -1.616429 & -0.933799 \\
\hline 6 & -5.141640 & -0.887087 & 1.322489 \\
\hline 6 & -5.430907 & -1.819661 & 0.325506 \\
\hline 8 & 0.426802 & -3.090786 & -1.690475 \\
\hline 1 & -5.571683 & -1.025401 & 2.313320 \\
\hline 1 & -5.070193 & -2.328258 & -1.728395 \\
\hline 1 & 5.418401 & -0.351120 & -2.543547 \\
\hline 1 & 5.333123 & -1.920125 & 1.431072 \\
\hline 6 & -4.062718 & 1.182516 & 2.240376 \\
\hline 1 & -3.443655 & 2.003982 & 1.867617 \\
\hline 6 & -3.543418 & -0.313720 & -2.639761 \\
\hline 1 & -2.714884 & 0.400289 & -2.595511 \\
\hline 6 & -6.360285 & -2.983137 & 0.646809 \\
\hline 1 & -7.290999 & -2.539003 & 1.027044 \\
\hline 6 & -3.279507 & 0.487256 & 3.360432 \\
\hline 1 & -2.318648 & 0.121168 & 2.989693 \\
\hline 1 & -3.090856 & 1.184319 & 4.182932 \\
\hline 1 & -3.841475 & -0.363462 & 3.762430 \\
\hline 6 & -5.362752 & 1.797279 & 2.771847 \\
\hline 1 & -5.913203 & 2.302236 & 1.973011 \\
\hline 1 & -6.017226 & 1.030912 & 3.199403 \\
\hline 1 & -5.147109 & 2.527188 & 3.557937 \\
\hline 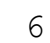 & -2.999617 & -1.593162 & -3.282692 \\
\hline
\end{tabular}




\begin{tabular}{|c|c|c|c|}
\hline L & -3.779238 & -2.350006 & -3.416210 \\
\hline 1 & -2.605607 & -1.359335 & -4.276744 \\
\hline 1 & -2.190295 & -2.022548 & -2.691232 \\
\hline 6 & -4.649693 & 0.274934 & -3.526996 \\
\hline & -4.276624 & 0.455007 & -4.540054 \\
\hline & -5.491777 & -0.422840 & -3.593634 \\
\hline 1 & -5.031262 & 1.218803 & -3.130111 \\
\hline 6 & -5.793948 & -3.870560 & 1.763976 \\
\hline & -4.881429 & -4.375447 & 1.428570 \\
\hline & -5.548561 & -3.293160 & 2.659611 \\
\hline & -6.518556 & -4.640561 & 2.045925 \\
\hline 6 & -6.718136 & -3.839771 & -0.565989 \\
\hline$\perp$ & -7.434492 & -4.614778 & -0.280236 \\
\hline 1 & -7.164115 & -3.243349 & -1.366645 \\
\hline 1 & -5.830281 & -4.340895 & -0.967926 \\
\hline 6 & 3.498144 & -0.278433 & 2.504396 \\
\hline 1 & 2.604305 & 0.350687 & 2.545640 \\
\hline 6 & 3.632076 & 1.625453 & -2.268182 \\
\hline 1 & 2.936611 & 2.340968 & -1.820682 \\
\hline 6 & 6.664511 & -2.158668 & -0.964821 \\
\hline 1 & 6.755896 & -2.141267 & -2.058626 \\
\hline 6 & 7.967644 & -1.603281 & -0.373941 \\
\hline 1 & 8.824161 & -2.215313 & -0.672706 \\
\hline 1 & 8.141505 & -0.575759 & -0.704104 \\
\hline 1 & 7.914994 & -1.600732 & 0.720039 \\
\hline 6 & 6.463310 & -3.612930 & -0.534102 \\
\hline 1 & 6.394761 & -3.699137 & 0.555639 \\
\hline 1 & 5.555359 & -4.043948 & -0.965198 \\
\hline 1 & 7.313551 & -4.221960 & -0.855040 \\
\hline 6 & 2.894902 & 0.930492 & -3.419245 \\
\hline 1 & 3.513520 & 0.145299 & -3.868407 \\
\hline 1 & 1.961489 & 0.484194 & -3.066221 \\
\hline 1 & 2.649568 & 1.652751 & -4.203914 \\
\hline 6 & 4.832577 & 2.427873 & -2.784950 \\
\hline 1 & 5.352966 & 2.926950 & -1.962745 \\
\hline 1 & 5.552974 & 1.782324 & -3.297569 \\
\hline 1 & 4.501512 & 3.189307 & -3.497445 \\
\hline 6 & 4.559026 & 0.314182 & 3.442213 \\
\hline 1 & 4.186167 & 0.347696 & 4.470367 \\
\hline 1 & 5.466728 & -0.299243 & 3.430603 \\
\hline 1 & 4.839315 & 1.328786 & 3.147898 \\
\hline 6 & 3.091250 & -1.672196 & 2.994882 \\
\hline 1 & 2.709741 & -1.601974 & 4.018138 \\
\hline 1 & 2.299180 & -2.090945 & 2.371801 \\
\hline 1 & 3.940278 & -2.364579 & 3.011523 \\
\hline 6 & 1.639636 & -2.563346 & -2.274298 \\
\hline 6 & 2.786671 & -3.344793 & -1.634246 \\
\hline 1 & 3.704564 & -2.755247 & -1.635579 \\
\hline 1 & 2.961115 & -4.294024 & -2.153383 \\
\hline 6 & 2.274178 & -3.609734 & -0.217208 \\
\hline 1 & 2.173558 & -2.643108 & 0.289380 \\
\hline 6 & 0.897943 & -4.157862 & -0.520158 \\
\hline 1 & 0.141943 & -2.449994 & 1.041347 \\
\hline 8 & -0.082192 & -3.417353 & 0.843993 \\
\hline 6 & -1.476573 & -3.766006 & 1.087387 \\
\hline 6 & -2.221632 & -3.912805 & -0.236865 \\
\hline 1 & -3.260289 & -4.192260 & -0.033580 \\
\hline 1 & -2.241651 & -2.955017 & -0.7616 \\
\hline
\end{tabular}









\begin{tabular}{|c|c|c|c|}
\hline 1 & -2.300230 & 6.492404 & -3.030124 \\
\hline 1 & -0.776466 & 5.042050 & -1.774118 \\
\hline 6 & -4.080866 & 5.364790 & -2.525655 \\
\hline 6 & -4.574977 & 4.264253 & -1.876732 \\
\hline 1 & -4.745581 & 6.017957 & -3.081485 \\
\hline 1 & -5.634238 & 4.025459 & -1.915542 \\
\hline 8 & 0.561082 & 0.977616 & -0.843187 \\
\hline 8 & -1.205635 & 0.796191 & 0.903728 \\
\hline 15 & -0.124921 & -0.118231 & 0.130246 \\
\hline 8 & 0.849349 & -0.586180 & 1.196989 \\
\hline 8 & -0.725050 & -1.181630 & -0.741976 \\
\hline 1 & 1.034840 & -1.835141 & 1.249508 \\
\hline 6 & 3.316708 & 1.302125 & -0.192538 \\
\hline 6 & 3.982615 & 0.876914 & 0.976072 \\
\hline 6 & 3.631493 & 0.713059 & -1.432834 \\
\hline 6 & 5.001124 & -0.074731 & 0.864884 \\
\hline 6 & 4.653215 & -0.233389 & -1.489426 \\
\hline 6 & 5.365238 & -0.629111 & -0.358680 \\
\hline 6 & -3.841879 & -0.039829 & 0.508127 \\
\hline 6 & -4.305252 & -0.969323 & -0.448327 \\
\hline 6 & -3.765583 & -0.404272 & 1.865569 \\
\hline 6 & -4.663583 & -2.248227 & -0.021016 \\
\hline 6 & -4.139633 & -1.694829 & 2.239982 \\
\hline 6 & -4.579300 & -2.635741 & 1.314708 \\
\hline 8 & 1.319116 & -2.922132 & 1.389949 \\
\hline 1 & -4.086523 & -1.978375 & 3.289075 \\
\hline 1 & -5.012293 & -2.968080 & -0.759802 \\
\hline 1 & 4.909432 & -0.679245 & -2.449823 \\
\hline 1 & 5.523942 & -0.381010 & 1.767639 \\
\hline 6 & -3.352511 & 0.573945 & 2.954894 \\
\hline 1 & -3.007688 & 1.497452 & 2.481986 \\
\hline 6 & -4.439119 & -0.648281 & -1.931689 \\
\hline 1 & -4.012880 & 0.339998 & -2.115437 \\
\hline 6 & -4.943169 & -4.042935 & 1.745938 \\
\hline 1 & -4.841689 & -4.084635 & 2.837787 \\
\hline 6 & -2.203250 & 0.036206 & 3.813833 \\
\hline 1 & -1.339376 & -0.224698 & 3.197825 \\
\hline 1 & -1.892380 & 0.793418 & 4.540382 \\
\hline 1 & -2.510289 & -0.851954 & 4.375697 \\
\hline 6 & -4.564287 & 0.935094 & 3.824263 \\
\hline 1 & -5.371558 & 1.355252 & 3.217362 \\
\hline 1 & -4.954463 & 0.049250 & 4.336101 \\
\hline 1 & -4.285487 & 1.669944 & 4.586026 \\
\hline 6 & -3.652128 & -1.630686 & -2.802088 \\
\hline 1 & -4.044188 & -2.650345 & -2.721874 \\
\hline 1 & -3.708197 & -1.333800 & -3.854366 \\
\hline 1 & -2.604882 & -1.638314 & -2.493622 \\
\hline 6 & -5.914976 & -0.608093 & -2.345467 \\
\hline 1 & -6.012042 & -0.336468 & -3.401316 \\
\hline 1 & -6.384863 & -1.587369 & -2.204146 \\
\hline 1 & -6.478661 & 0.116096 & -1.749402 \\
\hline 6 & -3.976048 & -5.067352 & 1.141695 \\
\hline 1 & -4.051487 & -5.065460 & 0.048365 \\
\hline 1 & -2.939119 & -4.839880 & 1.405388 \\
\hline 1 & -4.212154 & -6.076843 & 1.492783 \\
\hline 6 & -6.393789 & -4.388179 & 1.393301 \\
\hline 1 & -6.653525 & -5.384125 & 1.764885 \\
\hline 1 & -7.089351 & -3.664351 & 1.826072 \\
\hline
\end{tabular}




\begin{tabular}{|c|c|c|c|}
\hline 1 & -6.541950 & -4.389539 & 0.308360 \\
\hline 6 & 3.651690 & 1.407001 & 2.366794 \\
\hline 1 & 2.774329 & 2.053731 & 2.291803 \\
\hline 6 & 2.911216 & 1.071241 & -2.722459 \\
\hline 1 & 2.148140 & 1.821126 & -2.499969 \\
\hline 6 & 6.528185 & -1.592309 & -0.505550 \\
\hline 1 & 6.277445 & -2.275377 & -1.328468 \\
\hline 6 & 7.795169 & -0.824575 & -0.907965 \\
\hline 1 & 8.637023 & -1.508887 & -1.052963 \\
\hline$\perp$ & 7.638449 & -0.265599 & -1.834296 \\
\hline L & 8.064433 & -0.108796 & -0.124276 \\
\hline 6 & 6.788734 & -2.430070 & 0.745889 \\
\hline 1 & 7.193454 & -1.815742 & 1.556756 \\
\hline 1 & 5.868913 & -2.899332 & 1.109133 \\
\hline 1 & 7.519852 & -3.215584 & 0.535761 \\
\hline 6 & 2.202857 & -0.159950 & -3.302061 \\
\hline 1 & 2.931681 & -0.915580 & -3.617298 \\
\hline 1 & 1.535124 & -0.613326 & -2.563995 \\
\hline 1 & 1.607935 & 0.116161 & -4.178323 \\
\hline 6 & 3.873315 & 1.686437 & -3.745671 \\
\hline 1 & 4.358734 & 2.578717 & -3.340460 \\
\hline 1 & 4.656897 & 0.976293 & -4.029750 \\
\hline 1 & 3.333539 & 1.970070 & -4.654243 \\
\hline 6 & 4.811172 & 2.240556 & 2.926665 \\
\hline 1 & 4.546853 & 2.657295 & 3.903292 \\
\hline 1 & 5.707227 & 1.623769 & 3.056100 \\
\hline 1 & 5.073761 & 3.067782 & 2.260956 \\
\hline 6 & 3.283861 & 0.278972 & 3.337725 \\
\hline 1 & 3.055658 & 0.697230 & 4.323166 \\
\hline 1 & 2.402687 & -0.257105 & 2.978502 \\
\hline 1 & 4.107910 & -0.432532 & 3.464953 \\
\hline 6 & 2.615853 & -3.009704 & 1.990889 \\
\hline 6 & 2.628471 & -4.470050 & 0.082188 \\
\hline 6 & 1.147145 & -4.230263 & 0.356248 \\
\hline 1 & 0.059081 & -2.443144 & -0.813887 \\
\hline 8 & 0.583265 & -3.307820 & -1.022826 \\
\hline 6 & -0.197457 & -4.033138 & -2.028446 \\
\hline 6 & 0.509536 & -5.347108 & -2.342141 \\
\hline 1 & -0.019169 & -5.837832 & -3.164031 \\
\hline 1 & 1.540083 & -5.169879 & -2.664990 \\
\hline 1 & 0.504402 & -6.018807 & -1.481950 \\
\hline 6 & -0.193265 & -3.127933 & -3.258695 \\
\hline 1 & -0.812517 & -3.566936 & -4.046501 \\
\hline 1 & -0.584524 & -2.137933 & -3.013298 \\
\hline 1 & 0.827486 & -3.011503 & -3.636049 \\
\hline 6 & -1.606070 & -4.276827 & -1.490371 \\
\hline 1 & -2.040226 & -3.347395 & -1.108104 \\
\hline 1 & -2.248153 & -4.654375 & -2.292981 \\
\hline 1 & -1.578653 & -5.007025 & -0.680455 \\
\hline 6 & 3.181373 & -4.347360 & 1.507849 \\
\hline 1 & 2.803562 & -5.165874 & 2.126472 \\
\hline 1 & 4.272901 & -4.364493 & 1.530872 \\
\hline 1 & 2.509405 & -2.960623 & 3.077719 \\
\hline 1 & 3.228500 & -2.164889 & 1.65134 \\
\hline 8 & 0.336712 & -5.014794 & 0.735263 \\
\hline 1 & 2.712694 & -5.494338 & -0.291550 \\
\hline 6 & 3.292873 & -3.471511 & -0.86119 \\
\hline 1 & 4.379475 & -3.600661 & -0 \\
\hline
\end{tabular}




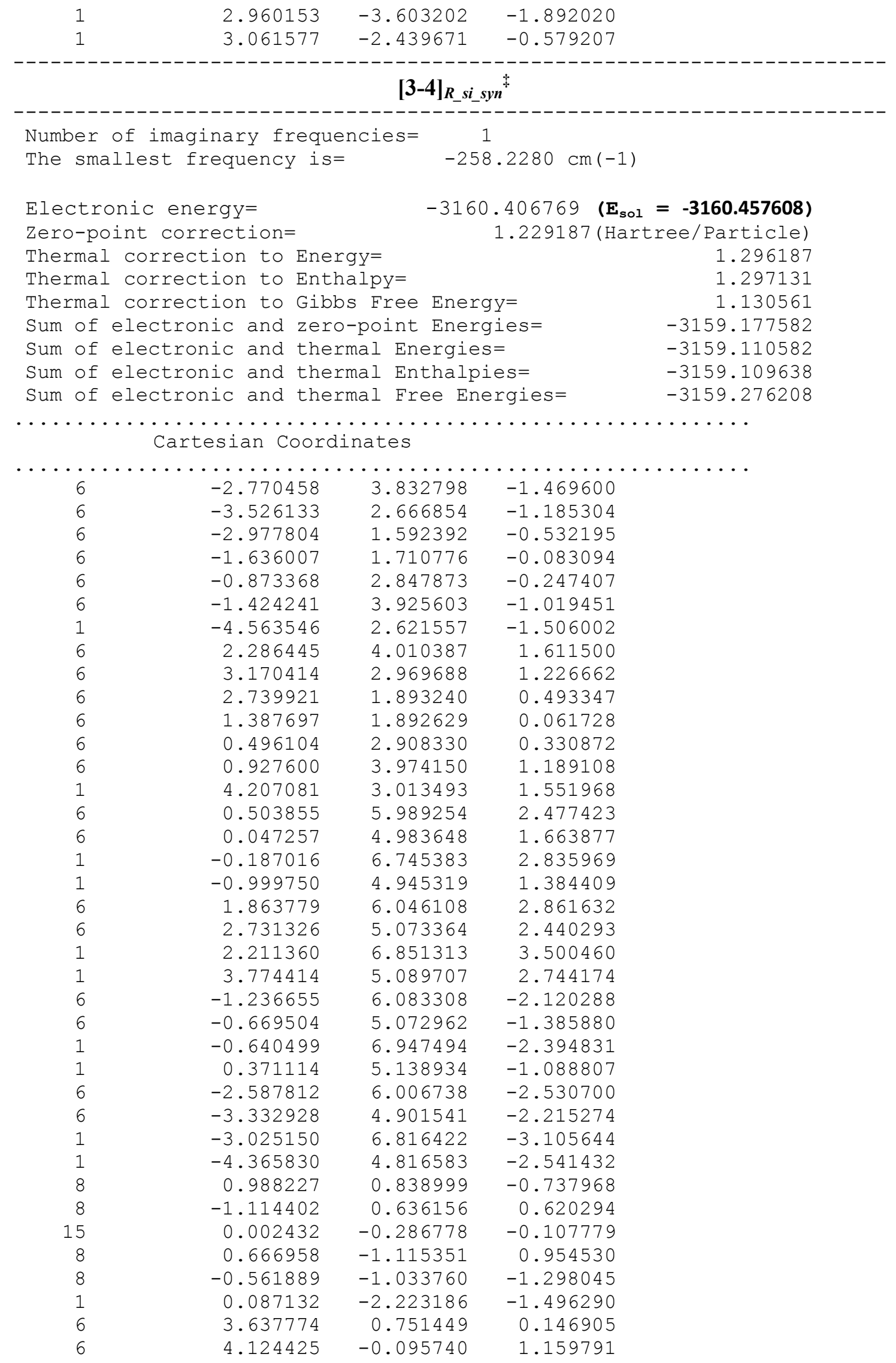




\begin{tabular}{|c|c|c|c|}
\hline 5 & 4.013312 & 0.538254 & -1.196495 \\
\hline 6 & 5.010059 & -1.123512 & 0.812445 \\
\hline 6 & 4.889340 & -0.503701 & -1.490294 \\
\hline 6 & 5.417037 & -1.336175 & -0.500807 \\
\hline 6 & -3.768721 & 0.356503 & -0.253084 \\
\hline 6 & -4.101340 & -0.523014 & -1.300860 \\
\hline 6 & -4.219180 & 0.108029 & 1.057653 \\
\hline 6 & -4.925793 & -1.613815 & -1.016038 \\
\hline 6 & -5.022390 & -1.007126 & 1.293219 \\
\hline 6 & -5.394161 & -1.876105 & 0.268711 \\
\hline 8 & 0.759494 & -3.055512 & -1.657188 \\
\hline 1 & -5.379894 & -1.190069 & 2.305084 \\
\hline 1 & -5.201203 & -2.294226 & -1.819370 \\
\hline 1 & 5.197176 & -0.661687 & -2.523773 \\
\hline 1 & 5.392818 & -1.764132 & 1.602574 \\
\hline 6 & -3.906131 & 1.041671 & 2.218763 \\
\hline 1 & -3.276595 & 1.856526 & 1.850117 \\
\hline 6 & -3.621005 & -0.303211 & -2.729175 \\
\hline 1 & -2.807647 & 0.428367 & -2.700805 \\
\hline 6 & -6.322228 & -3.047922 & 0.531818 \\
\hline 1 & -6.318440 & -3.674311 & -0.369540 \\
\hline 6 & -3.126580 & 0.331077 & 3.330520 \\
\hline 1 & -2.160478 & -0.022405 & 2.96195 \\
\hline 1 & -2.946209 & 1.017882 & 4.163393 \\
\hline 1 & -3.685131 & -0.527463 & 3.719957 \\
\hline 6 & -5.193420 & 1.673319 & 2.763491 \\
\hline 1 & -5.739055 & 2.194440 & 1.971707 \\
\hline 1 & -5.858108 & 0.912203 & 3.184995 \\
\hline 1 & -4.962178 & 2.391923 & 3.555633 \\
\hline 6 & -3.056681 & -1.573775 & -3.373102 \\
\hline 1 & -3.818894 & -2.352604 & -3.477841 \\
\hline 1 & -2.689210 & -1.341446 & -4.377481 \\
\hline 1 & -2.223907 & -1.970104 & -2.790347 \\
\hline 6 & -4.755429 & 0.263148 & -3.593541 \\
\hline 1 & -4.404577 & 0.453999 & -4.612469 \\
\hline 1 & -5.585239 & -0.450043 & -3.648875 \\
\hline 1 & -5.148298 & 1.198481 & -3.186259 \\
\hline 6 & -7.755111 & -2.549273 & 0.757479 \\
\hline 1 & -7.800287 & -1.915742 & 1.649798 \\
\hline 1 & -8.101457 & -1.955942 & -0.092815 \\
\hline 1 & -8.443472 & -3.387938 & 0.900738 \\
\hline 6 & -5.865471 & -3.914931 & 1.708832 \\
\hline 1 & -6.538454 & -4.767954 & 1.837263 \\
\hline 1 & -4.853227 & -4.300758 & 1.554721 \\
\hline 1 & -5.870953 & -3.347059 & 2.64484 \\
\hline 6 & 3.776461 & 0.094919 & 2.630896 \\
\hline 1 & 3.016682 & 0.876448 & 2.703965 \\
\hline 6 & 3.551460 & 1.443562 & -2.329398 \\
\hline 1 & 2.862111 & 2.187691 & -1.921705 \\
\hline 6 & 6.466072 & -2.364865 & -0.892207 \\
\hline 1 & 6.144652 & -2.808520 & -1.845559 \\
\hline 6 & 7.807399 & -1.658902 & -1.139981 \\
\hline 1 & 8.566813 & -2.372119 & -1.47497 \\
\hline 1 & 7.708736 & -0.875863 & -1.896291 \\
\hline 1 & 8.158910 & -1.190249 & -0.214990 \\
\hline 6 & 6.649387 & -3.496582 & 0.11735 \\
\hline 1 & 7.055681 & -3.120946 & 1.061963 \\
\hline 1 & 5.707935 & -4.010570 & 0.333888 \\
\hline
\end{tabular}




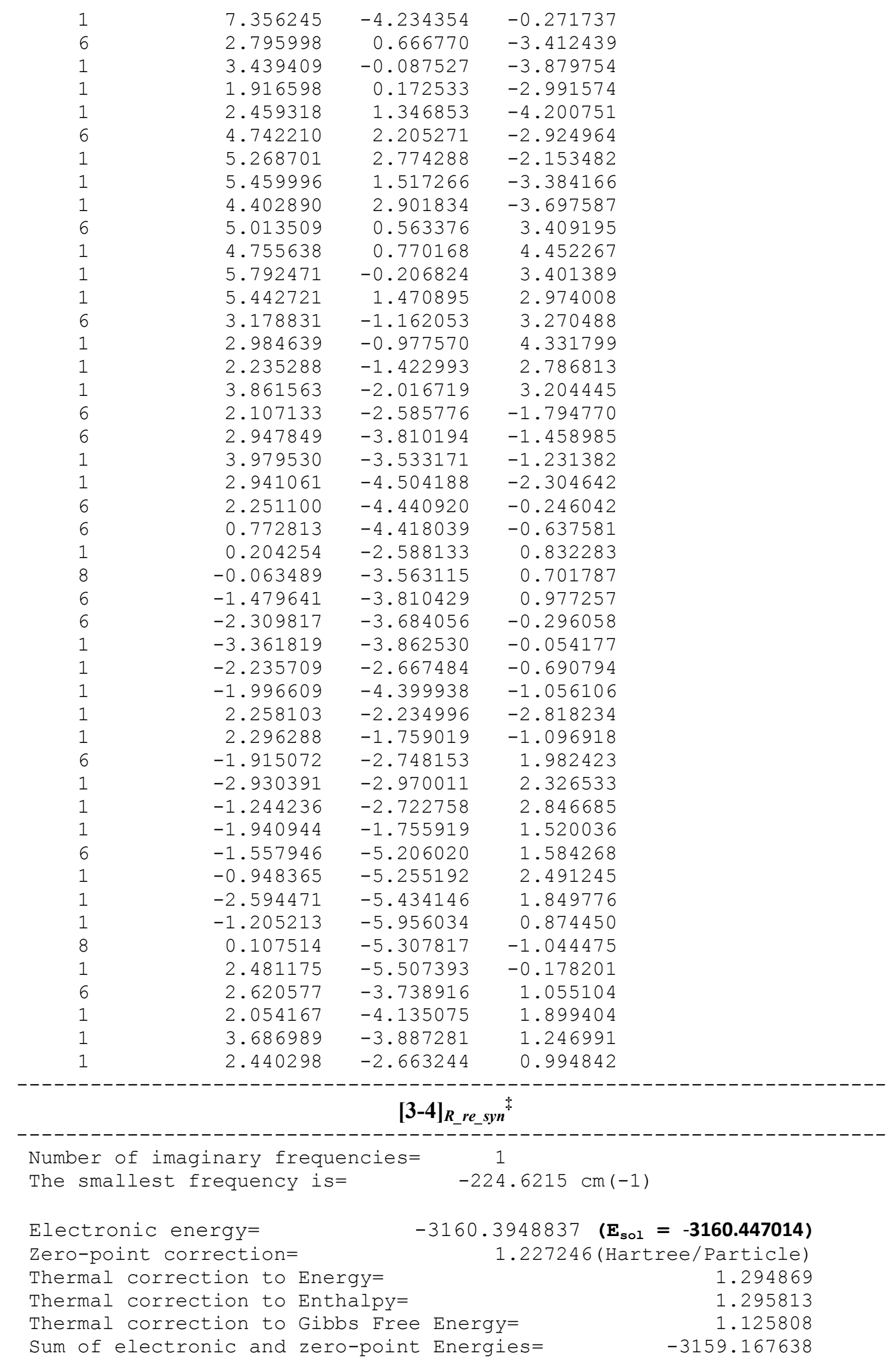


$\begin{array}{ll}\text { Sum of electronic and thermal Energies= } & -3159.100015 \\ \text { Sum of electronic and thermal Enthalpies= } & -3159.099071 \\ \text { Sum of electronic and thermal Eree Energies= } & -3159.269076\end{array}$

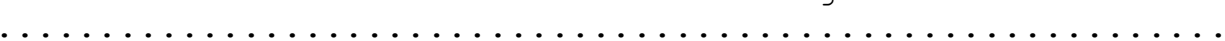

Cartesian Coordinates

\begin{tabular}{|c|c|c|c|}
\hline 6 & 3.617625 & 2.918094 & 1.798649 \\
\hline 6 & 4.183740 & 1.829255 & 1.087178 \\
\hline 6 & 3.431885 & 1.036119 & 0.256048 \\
\hline 6 & 2.068280 & 1.389351 & 0.078869 \\
\hline 6 & 1.483485 & 2.499838 & 0.648936 \\
\hline 6 & 2.250388 & 3.253672 & 1.597586 \\
\hline 1 & 5.238572 & 1.604515 & 1.222691 \\
\hline 6 & -1.537684 & 4.517336 & -0.582256 \\
\hline 6 & -2.536709 & 3.518148 & -0.485656 \\
\hline 6 & -2.244646 & 2.230087 & -0.109455 \\
\hline 6 & -0.911571 & 1.948029 & 0.286612 \\
\hline 6 & 0.103262 & 2.883052 & 0.249434 \\
\hline 6 & -0.190862 & 4.192247 & -0.261293 \\
\hline 1 & -3.558747 & 3.768419 & -0.758768 \\
\hline 6 & 0.474789 & 6.423125 & -0.958860 \\
\hline 6 & 0.808584 & 5.177832 & -0.490735 \\
\hline 1 & 1.254621 & 7.157944 & -1.130509 \\
\hline 1 & 1.847596 & 4.934624 & -0.301887 \\
\hline 6 & -0.872862 & 6.758149 & -1.228165 \\
\hline 6 & -1.854071 & 5.819789 & -1.050111 \\
\hline 1 & -1.121442 & 7.750145 & -1.590771 \\
\hline 1 & -2.891460 & 6.049040 & -1.278129 \\
\hline 6 & 2.458550 & 5.006881 & 3.263910 \\
\hline 6 & 1.690351 & 4.306919 & 2.368865 \\
\hline 1 & 2.010356 & 5.802731 & 3.849937 \\
\hline 1 & 0.639249 & 4.545798 & 2.249169 \\
\hline 6 & 3.827865 & 4.696903 & 3.435473 \\
\hline 6 & 4.390182 & 3.672402 & 2.720797 \\
\hline 1 & 4.425289 & 5.264172 & 4.141637 \\
\hline 1 & 5.435925 & 3.410232 & 2.855682 \\
\hline 8 & -0.652761 & 0.686801 & 0.791040 \\
\hline 8 & 1.315232 & 0.592269 & -0.773310 \\
\hline 15 & 0.229477 & -0.372504 & -0.066807 \\
\hline 8 & -0.608290 & -0.984099 & -1.174767 \\
\hline 8 & 0.834629 & -1.342311 & 0.906807 \\
\hline 1 & -0.708762 & -2.281344 & -0.978700 \\
\hline 6 & -3.314037 & 1.189074 & -0.197607 \\
\hline 6 & -3.772828 & 0.803647 & -1.473340 \\
\hline 6 & -3.945168 & 0.694394 & 0.956255 \\
\hline 6 & -4.924609 & 0.020064 & -1.566030 \\
\hline 6 & -5.087201 & -0.095059 & 0.812891 \\
\hline 6 & -5.613885 & -0.417189 & -0.436550 \\
\hline 6 & 4.022220 & -0.137470 & -0.454257 \\
\hline 6 & 4.486031 & -1.244693 & 0.284345 \\
\hline 6 & 4.151178 & -0.120108 & -1.856019 \\
\hline 6 & 5.113637 & -2.289454 & -0.393553 \\
\hline 6 & 4.774176 & -1.197628 & -2.487421 \\
\hline 6 & 5.271061 & -2.285710 & -1.777737 \\
\hline 8 & -0.906612 & -3.370902 & -0.932336 \\
\hline 1 & 4.882813 & -1.185674 & -3.570233 \\
\hline 1 & 5.480547 & -3.139367 & 0.178638 \\
\hline 1 & -5.594909 & -0.459780 & 1.704997 \\
\hline
\end{tabular}




\begin{tabular}{|c|c|c|c|}
\hline 1 & -5.297874 & -0.241329 & -2.553840 \\
\hline 6 & 3.668899 & 1.038377 & -2.716510 \\
\hline 1 & 3.185770 & 1.778293 & -2.072597 \\
\hline 6 & 4.304318 & -1.364403 & 1.790899 \\
\hline & 3.649321 & -0.553674 & 2.122590 \\
\hline 6 & 5.955234 & -3.436885 & -2.489501 \\
\hline 1 & 5.937619 & -3.211760 & -3.563462 \\
\hline 6 & 2.630470 & 0.576738 & -3.745447 \\
\hline 1 & 1.785585 & 0.087769 & -3.254487 \\
\hline 1 & 2.253793 & 1.434067 & -4.312112 \\
\hline 1 & 3.070477 & -0.126443 & -4.460536 \\
\hline 6 & 4.849930 & 1.737817 & -3.400839 \\
\hline 1 & 5.575511 & 2.093806 & -2.663954 \\
\hline 1 & 5.369141 & 1.056400 & -4.082542 \\
\hline 1 & 4.501153 & 2.595195 & -3.984774 \\
\hline 6 & 3.605142 & -2.678003 & 2.155171 \\
\hline 1 & 4.225874 & -3.548792 & 1.920094 \\
\hline 1 & 3.387558 & -2.708356 & 3.228293 \\
\hline 1 & 2.666154 & -2.754681 & 1.603158 \\
\hline 6 & 5.643863 & -1.240478 & 2.525492 \\
\hline 1 & 5.499384 & -1.320634 & 3.607421 \\
\hline 1 & 6.327082 & -2.038836 & 2.216114 \\
\hline 1 & 6.134898 & -0.285828 & 2.315529 \\
\hline 6 & 5.206114 & -4.755723 & -2.270776 \\
\hline 1 & 5.215192 & -5.036802 & -1.212541 \\
\hline 1 & 4.162818 & -4.673434 & -2.585964 \\
\hline 1 & 5.677823 & -5.565921 & -2.835363 \\
\hline 6 & 7.419952 & -3.564765 & -2.057266 \\
\hline 1 & 7.918325 & -4.368278 & -2.608225 \\
\hline 1 & 7.964696 & -2.633369 & -2.232827 \\
\hline 1 & 7.488784 & -3.797962 & -0.989485 \\
\hline 6 & -3.062293 & 1.212575 & -2.756710 \\
\hline 1 & -2.117786 & 1.693498 & -2.485262 \\
\hline 6 & -3.431705 & 0.998760 & 2.353062 \\
\hline 1 & -2.510061 & 1.580231 & 2.262924 \\
\hline 6 & -6.938216 & -1.152034 & -0.542197 \\
\hline 1 & -7.109835 & -1.652743 & 0.420392 \\
\hline 6 & -8.072676 & -0.139542 & -0.750987 \\
\hline 1 & -9.044303 & -0.642385 & -0.779898 \\
\hline 1 & -8.087887 & 0.603366 & 0.050782 \\
\hline 1 & -7.933095 & 0.390530 & -1.699056 \\
\hline 6 & -6.966663 & -2.216670 & -1.641310 \\
\hline 1 & -6.875272 & -1.765110 & -2.634200 \\
\hline 1 & -6.156839 & -2.942672 & -1.525427 \\
\hline 1 & -7.916304 & -2.758868 & -1.616829 \\
\hline 6 & -3.083816 & -0.301272 & 3.087596 \\
\hline 1 & -3.984410 & -0.896872 & 3.277882 \\
\hline 1 & -2.389240 & -0.909304 & 2.497679 \\
\hline 1 & -2.620123 & -0.082711 & 4.055261 \\
\hline 6 & -4.436510 & 1.838676 & 3.14844 \\
\hline 1 & -4.645748 & 2.783555 & 2.638760 \\
\hline 1 & -5.384777 & 1.305263 & 3.27184 \\
\hline 1 & -4.045051 & 2.064035 & 4.145105 \\
\hline 6 & -3.896105 & 2.213464 & -3.564590 \\
\hline 1 & -3.362569 & 2.512251 & -4.47187 \\
\hline 1 & -4.851006 & 1.768774 & -3.865711 \\
\hline 1 & -4.115152 & 3.114555 & -2.98518 \\
\hline 6 & -2.708653 & -0.011346 & -3.60975 \\
\hline
\end{tabular}




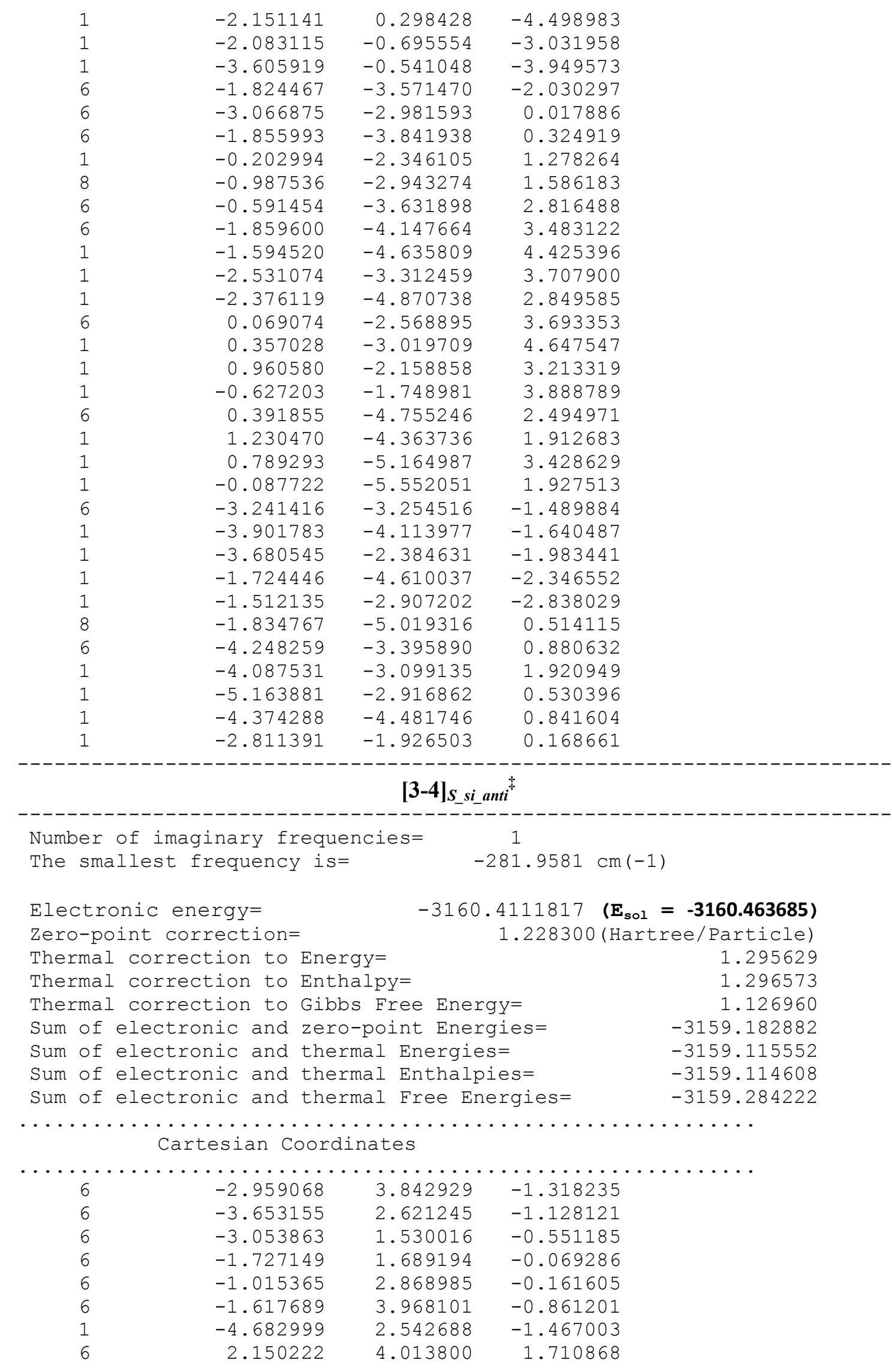




\begin{tabular}{|c|c|c|c|}
\hline 6 & 3.057157 & 3.028973 & 1.239277 \\
\hline 6 & 2.645821 & 1.994127 & 0.437493 \\
\hline 6 & 1.285963 & 1.991816 & 0.032801 \\
\hline 6 & 0.364213 & 2.949283 & 0.395509 \\
\hline 6 & 0.783549 & 3.967616 & 1.314020 \\
\hline 1 & 4.098050 & 3.084524 & 1.547629 \\
\hline 6 & 0.332205 & 5.881631 & 2.739062 \\
\hline 6 & -0.113893 & 4.918353 & 1.870296 \\
\hline 1 & -0.370612 & 6.595104 & 3.156907 \\
\hline 1 & -1.164403 & 4.868938 & 1.605849 \\
\hline 6 & 1.697746 & 5.950212 & 3.101219 \\
\hline 6 & 2.582389 & 5.031918 & 2.600398 \\
\hline 1 & 2.037588 & 6.721399 & 3.784655 \\
\hline 1 & 3.630648 & 5.058209 & 2.885158 \\
\hline 6 & -1.534697 & 6.201134 & -1.815732 \\
\hline 6 & -0.918432 & 5.171289 & -1.151757 \\
\hline 1 & -0.979679 & 7.107829 & -2.033599 \\
\hline 1 & 0.119395 & 5.265896 & -0.853156 \\
\hline 6 & -2.882600 & 6.090186 & -2.228999 \\
\hline 6 & -3.574003 & 4.932490 & -1.988993 \\
\hline 1 & -3.359049 & 6.915423 & -2.747906 \\
\hline 1 & -4.602440 & 4.820514 & -2.321019 \\
\hline 8 & 0.891397 & 0.978511 & -0.820639 \\
\hline 8 & -1.160731 & 0.602166 & 0.579172 \\
\hline 15 & 0.023470 & -0.219322 & -0.163053 \\
\hline 8 & 0.799530 & -0.934457 & 0.915721 \\
\hline 8 & -0.477811 & -1.052592 & -1.312786 \\
\hline 1 & 0.304662 & -2.205160 & -1.405378 \\
\hline 6 & 3.576689 & 0.909311 & -0.003692 \\
\hline 6 & 4.129521 & 0.020436 & 0.945333 \\
\hline 6 & 3.937964 & 0.801375 & -1.360447 \\
\hline 6 & 5.082483 & -0.908764 & 0.520873 \\
\hline 6 & 4.904670 & -0.136414 & -1.729576 \\
\hline 6 & 5.512587 & -0.980207 & -0.805313 \\
\hline 6 & -3.785294 & 0.231804 & -0.407444 \\
\hline 6 & -3.857039 & -0.659145 & -1.494799 \\
\hline 6 & -4.436841 & -0.069619 & 0.802271 \\
\hline 6 & -4.624447 & -1.818078 & -1.357183 \\
\hline 6 & -5.193249 & -1.238354 & 0.889118 \\
\hline 6 & -5.309808 & -2.120449 & -0.183704 \\
\hline 8 & 0.975758 & -3.026183 & -1.364540 \\
\hline 1 & -5.701416 & -1.466595 & 1.824694 \\
\hline 1 & -4.691624 & -2.510827 & -2.192379 \\
\hline 1 & 5.215789 & -0.190737 & -2.771024 \\
\hline 1 & 5.528411 & -1.573658 & 1.259243 \\
\hline 6 & -4.313774 & 0.820488 & 2.028940 \\
\hline 1 & -3.755424 & 1.718966 & 1.748141 \\
\hline 6 & -3.155275 & -0.376568 & -2.814725 \\
\hline 1 & -2.309318 & 0.286046 & -2.605737 \\
\hline 6 & -6.154054 & -3.377169 & -0.077467 \\
\hline 1 & -6.125067 & -3.870454 & -1.057111 \\
\hline 6 & -3.513809 & 0.104735 & 3.124088 \\
\hline 1 & -2.522637 & -0.171421 & 2.753587 \\
\hline 1 & -3.390913 & 0.750613 & 3.999281 \\
\hline 1 & -4.027079 & -0.808691 & 3.446685 \\
\hline 6 & -5.682666 & 1.275060 & 2.545169 \\
\hline 1 & -6.245708 & 1.789749 & 1.761560 \\
\hline 1 & -6.281577 & 0.424644 & 2.886568 \\
\hline
\end{tabular}




\begin{tabular}{|c|c|c|c|}
\hline 1 & -5.564071 & 1.958892 & 3.391093 \\
\hline 6 & -2.592211 & -1.642460 & -3.468961 \\
\hline 1 & -3.387612 & -2.253542 & -3.909354 \\
\hline 1 & -1.911269 & -1.364672 & -4.278665 \\
\hline & -2.038915 & -2.247870 & -2.748883 \\
\hline & -4.101978 & 0.334390 & -3.791236 \\
\hline & -3.597072 & 0.522581 & -4.744053 \\
\hline & -4.980034 & -0.290130 & -3.989484 \\
\hline & -4.450745 & 1.291955 & -3.396748 \\
\hline 6 & -7.615716 & -3.041823 & 0.238942 \\
\hline 1 & -7.703691 & -2.573746 & 1.224914 \\
\hline 1 & -8.028995 & -2.348828 & -0.49846 \\
\hline 1 & -8.227654 & -3.948923 & 0.244928 \\
\hline 6 & -5.585411 & -4.355130 & 0.956689 \\
\hline 1 & -6.202168 & -5.257376 & 1.014803 \\
\hline 1 & -4.564351 & -4.650903 & 0.700623 \\
\hline 1 & -5.565771 & -3.897411 & 1.952534 \\
\hline 6 & 3.777710 & 0.080363 & 2.42728 \\
\hline 1 & 2.939873 & 0.771326 & 2.551226 \\
\hline 6 & 3.352201 & 1.689127 & -2.448277 \\
\hline 1 & 2.616396 & 2.359488 & -1.997494 \\
\hline 6 & 6.665469 & -1.876751 & -1.223533 \\
\hline 1 & 6.823792 & -1.714745 & -2.29714 \\
\hline 6 & 7.951683 & -1.470219 & -0.493827 \\
\hline 1 & 8.801972 & -2.060005 & -0.849471 \\
\hline 1 & 8.172945 & -0.411484 & -0.65057 \\
\hline 1 & 7.854318 & -1.636987 & 0.584029 \\
\hline 6 & 6.379292 & -3.365856 & -1.016153 \\
\hline 1 & 6.186661 & -3.583808 & 0.040303 \\
\hline 1 & 5.510183 & -3.687015 & -1.598371 \\
\hline 1 & 7.237873 & -3.968508 & -1.32744 \\
\hline 6 & 2.626188 & 0.851032 & -3.50692 \\
\hline 1 & 3.322475 & 0.182399 & -4.02447 \\
\hline 1 & 1.836352 & 0.247698 & -3.051459 \\
\hline 1 & 2.170213 & 1.502623 & -4.258526 \\
\hline 6 & 4.435629 & 2.569781 & -3.082958 \\
\hline 1 & 4.940351 & 3.176554 & -2.32573 \\
\hline 1 & 5.193946 & 1.963932 & -3.589144 \\
\hline 1 & 3.994662 & 3.241462 & -3.825490 \\
\hline 6 & 4.968745 & 0.616015 & 3.23399 \\
\hline 1 & 4.699496 & 0.729914 & 4.288490 \\
\hline 1 & 5.816387 & -0.075693 & 3.175041 \\
\hline 1 & 5.309883 & 1.585318 & 2.859462 \\
\hline 6 & 3.324117 & -1.271061 & 2.98859 \\
\hline 1 & 3.125885 & -1.177898 & 4.06121 \\
\hline 1 & 2.404137 & -1.596151 & 2.499108 \\
\hline 1 & 4.091335 & -2.042945 & 2.862171 \\
\hline 6 & 2.348972 & -2.662580 & -1.188672 \\
\hline 6 & 2.844587 & -3.523747 & -0.018972 \\
\hline 6 & 1.982571 & -4.784198 & -0.02859 \\
\hline 6 & 0.555887 & -4.338320 & -0.276815 \\
\hline 1 & 0.521266 & -2.336560 & 0.995640 \\
\hline 8 & 0.279640 & -3.350104 & 1.11279 \\
\hline 6 & -0.964180 & -3.459324 & 1.881062 \\
\hline 6 & -2.133999 & -2.913594 & 1.072125 \\
\hline 1 & -3.053052 & -3.013635 & 1.65755 \\
\hline 1 & -2.013568 & -1.848995 & 0.845826 \\
\hline 1 & -2.252712 & -3.457827 & 0.13264 \\
\hline
\end{tabular}









\begin{tabular}{|c|c|c|c|}
\hline 6 & 1.202675 & 5.640662 & 2.949563 \\
\hline 6 & 0.625024 & 4.721958 & 2.110434 \\
\hline 1 & 0.576899 & 6.354474 & 3.475400 \\
\hline 1 & -0.451067 & 4.712834 & 1.980191 \\
\hline 6 & 2.602908 & 5.662145 & 3.143468 \\
\hline 6 & 3.389523 & 4.735249 & 2.512588 \\
\hline 1 & 3.046220 & 6.400193 & 3.803858 \\
\hline 1 & 4.464672 & 4.720086 & 2.668573 \\
\hline 8 & -0.972849 & 0.629611 & 0.558174 \\
\hline 8 & 1.172663 & 0.795556 & -0.752592 \\
\hline 15 & 0.052594 & -0.279978 & -0.310570 \\
\hline 8 & -0.531879 & -0.752672 & -1.618404 \\
\hline 8 & 0.516769 & -1.341375 & 0.651795 \\
\hline 1 & -0.868742 & -2.074383 & -1.564170 \\
\hline 6 & -3.722522 & 0.774521 & -0.097565 \\
\hline 6 & -4.365090 & 0.311907 & -1.269924 \\
\hline 6 & -3.966751 & 0.136785 & 1.134471 \\
\hline 6 & -5.275287 & -0.739779 & -1.146773 \\
\hline 6 & -4.880210 & -0.920154 & 1.193149 \\
\hline 6 & -5.561356 & -1.366466 & 0.066196 \\
\hline 6 & 3.917670 & 0.631103 & -0.235054 \\
\hline 6 & 4.610640 & -0.230273 & 0.643618 \\
\hline 6 & 4.026755 & 0.449679 & -1.627913 \\
\hline 6 & 5.369048 & -1.270947 & 0.106975 \\
\hline 6 & 4.809348 & -0.599271 & -2.112205 \\
\hline 6 & 5.482484 & -1.474397 & -1.266985 \\
\hline 8 & -1.352676 & -3.009689 & -1.372579 \\
\hline 1 & 4.904011 & -0.734681 & -3.187679 \\
\hline 1 & 5.886651 & -1.946391 & 0.785103 \\
\hline 1 & -5.058734 & -1.392980 & 2.154395 \\
\hline 1 & -5.765795 & -1.102045 & -2.050270 \\
\hline 6 & 3.389641 & 1.393750 & -2.637811 \\
\hline 1 & 2.772236 & 2.118198 & -2.100486 \\
\hline 6 & 4.503666 & -0.126364 & 2.159825 \\
\hline 1 & 4.081233 & 0.845937 & 2.417474 \\
\hline 6 & 6.340562 & -2.593361 & -1.825125 \\
\hline 1 & 6.242053 & -2.563770 & -2.917550 \\
\hline 6 & 2.482671 & 0.655805 & -3.627775 \\
\hline 1 & 1.695435 & 0.104727 & -3.108505 \\
\hline 1 & 2.010164 & 1.372567 & -4.306692 \\
\hline 1 & 3.056071 & -0.047808 & -4.240604 \\
\hline 6 & 4.477056 & 2.186874 & -3.375321 \\
\hline 1 & 5.108476 & 2.737498 & -2.671863 \\
\hline 1 & 5.122541 & 1.519974 & -3.956265 \\
\hline 1 & 4.024828 & 2.903501 & -4.067755 \\
\hline 6 & 3.526871 & -1.187232 & 2.670096 \\
\hline 1 & 3.890883 & -2.191240 & 2.421933 \\
\hline 1 & 3.404920 & -1.124790 & 3.756921 \\
\hline 1 & 2.548739 & -1.056828 & 2.196833 \\
\hline 6 & 5.860919 & -0.228789 & 2.861285 \\
\hline 1 & 5.742397 & -0.040857 & 3.932583 \\
\hline 1 & 6.303200 & -1.224039 & 2.753326 \\
\hline 1 & 6.572210 & 0.499206 & 2.460269 \\
\hline 6 & 5.864998 & -3.968615 & -1.347129 \\
\hline 1 & 5.963198 & -4.057142 & -0.259408 \\
\hline 1 & 4.816215 & -4.135716 & -1.605910 \\
\hline 1 & 6.465289 & -4.763507 & -1.800434 \\
\hline 6 & 7.818003 & -2.379106 & -1.476918 \\
\hline
\end{tabular}




\begin{tabular}{|c|c|c|c|}
\hline 1 & 8.438792 & -3.162465 & -1.922666 \\
\hline 1 & 8.171226 & -1.409440 & -1.837274 \\
\hline 1 & 7.966343 & -2.408664 & -0.392285 \\
\hline 0 & -4.155647 & 0.829367 & -2.697786 \\
\hline & -4.392086 & -0.041316 & -3.322834 \\
\hline & -3.293497 & 0.557363 & 2.432896 \\
\hline & -2.609084 & 1.382856 & 2.223715 \\
\hline & -6.589224 & -2.487011 & 0.101319 \\
\hline & -6.298120 & -3.214893 & -0.670778 \\
\hline & -6.664009 & -3.220432 & 1.439555 \\
\hline & -7.364526 & -4.056764 & 1.369712 \\
\hline & -5.692826 & -3.614619 & 1.753409 \\
\hline & -7.024974 & -2.551874 & 2.228480 \\
\hline 6 & -7.977470 & -1.945031 & -0.267808 \\
\hline & -8.300293 & -1.209135 & 0.475772 \\
\hline & -7.973329 & -1.454359 & -1.244165 \\
\hline D. & -8.714519 & -2.753409 & -0.293091 \\
\hline 6 & -2.466291 & -0.593045 & 3.020748 \\
\hline 1 & -3.108762 & -1.436858 & 3.297305 \\
\hline & -1.716011 & -0.941026 & 2.304920 \\
\hline & -1.946563 & -0.262598 & 3.925377 \\
\hline 6 & -4.323933 & 1.069602 & 3.446854 \\
\hline T. & -4.901655 & 1.901380 & 3.033857 \\
\hline 1 & -5.026262 & 0.279902 & 3.732686 \\
\hline 1 & -3.822810 & 1.415263 & 4.356061 \\
\hline 6 & -2.737585 & 1.251438 & -3.101498 \\
\hline 1 & -2.679121 & 1.264995 & -4.194691 \\
\hline & -2.484896 & 2.255312 & -2.753164 \\
\hline 1 & -1.984402 & 0.552820 & -2.729448 \\
\hline 6 & -5.178526 & 1.909663 & -3.078776 \\
\hline 1 & -5.148047 & 2.085893 & -4.158561 \\
\hline 1 & -6.195805 & 1.613287 & -2.806334 \\
\hline 1 & -4.952351 & 2.859200 & -2.586613 \\
\hline 6 & -2.640434 & -2.747839 & -0.819710 \\
\hline 6 & -2.828858 & -3.789476 & 0.277964 \\
\hline 1 & -3.886156 & -4.022558 & 0.428670 \\
\hline 1 & -2.416416 & -3.432987 & 1.228389 \\
\hline 6 & -2.050502 & -5.010326 & -0.192120 \\
\hline 5 & -0.706730 & -4.542293 & -0.729540 \\
\hline 1 & 0.134343 & -2.784212 & 0.555832 \\
\hline 8 & 0.043371 & -3.817107 & 0.625292 \\
\hline 6 & 1.350833 & -4.301924 & 1.119538 \\
\hline 6 & 1.394183 & -5.825612 & 1.111145 \\
\hline 1 & 2.361533 & -6.126978 & 1.522733 \\
\hline 1 & 0.618402 & -6.248889 & 1.751607 \\
\hline 1 & 1.304339 & -6.236095 & 0.105459 \\
\hline 1 & -2.681802 & -1.721592 & -0.441278 \\
\hline 1 & -3.392030 & -2.862109 & -1.608763 \\
\hline 6 & 1.403139 & -3.795043 & 2.557672 \\
\hline 1 & 2.371252 & -4.037359 & 3.005495 \\
\hline 1 & 1.261281 & -2.713763 & 2.590874 \\
\hline 1 & 0.615113 & -4.272181 & 3.147996 \\
\hline 6 & 2.461283 & -3.727926 & 0.242833 \\
\hline 1 & 2.458012 & -2.635497 & 0.252164 \\
\hline 1 & 3.431272 & -4.070987 & 0.617122 \\
\hline 1 & 2.339131 & -4.081167 & -0.784562 \\
\hline B & 0.059375 & -5.062245 & -1.46476 \\
\hline & -2.532243 & -5.423998 & -1.0884 \\
\hline
\end{tabular}




$\begin{array}{cccc}6 & -1.921155 & -6.103318 & 0.859459 \\ 1 & -2.911513 & -6.507356 & 1.084420 \\ 1 & -1.287734 & -6.921940 & 0.509520 \\ 1 & -1.501566 & -5.695856 & 1.782756 \\ - & {[3-4]_{R_{-} \text {si_anti }}{ }^{\ddagger}}\end{array}$

Number of imaginary frequencies=

The smallest frequency is=

1

$-278.8745 \mathrm{~cm}(-1)$

Electronic energy=

$-3160.4115346 \quad\left(\mathbf{E}_{\mathrm{sol}}=\mathbf{- 3 1 6 0 . 4 6 4 1 5 2}\right)$

Zero-point correction= 1.228953 (Hartree/Particle)

Thermal correction to Energy=

1.295991

Thermal correction to Enthalpy=

1.296935

Thermal correction to Gibbs Free Energy=

1.128719

Sum of electronic and zero-point Energies=

$-3159.182582$

Sum of electronic and thermal Energies=

$-3159.115544$

Sum of electronic and thermal Enthalpies=

$-3159.114599$

Sum of electronic and thermal Free Energies=

$-3159.282816$

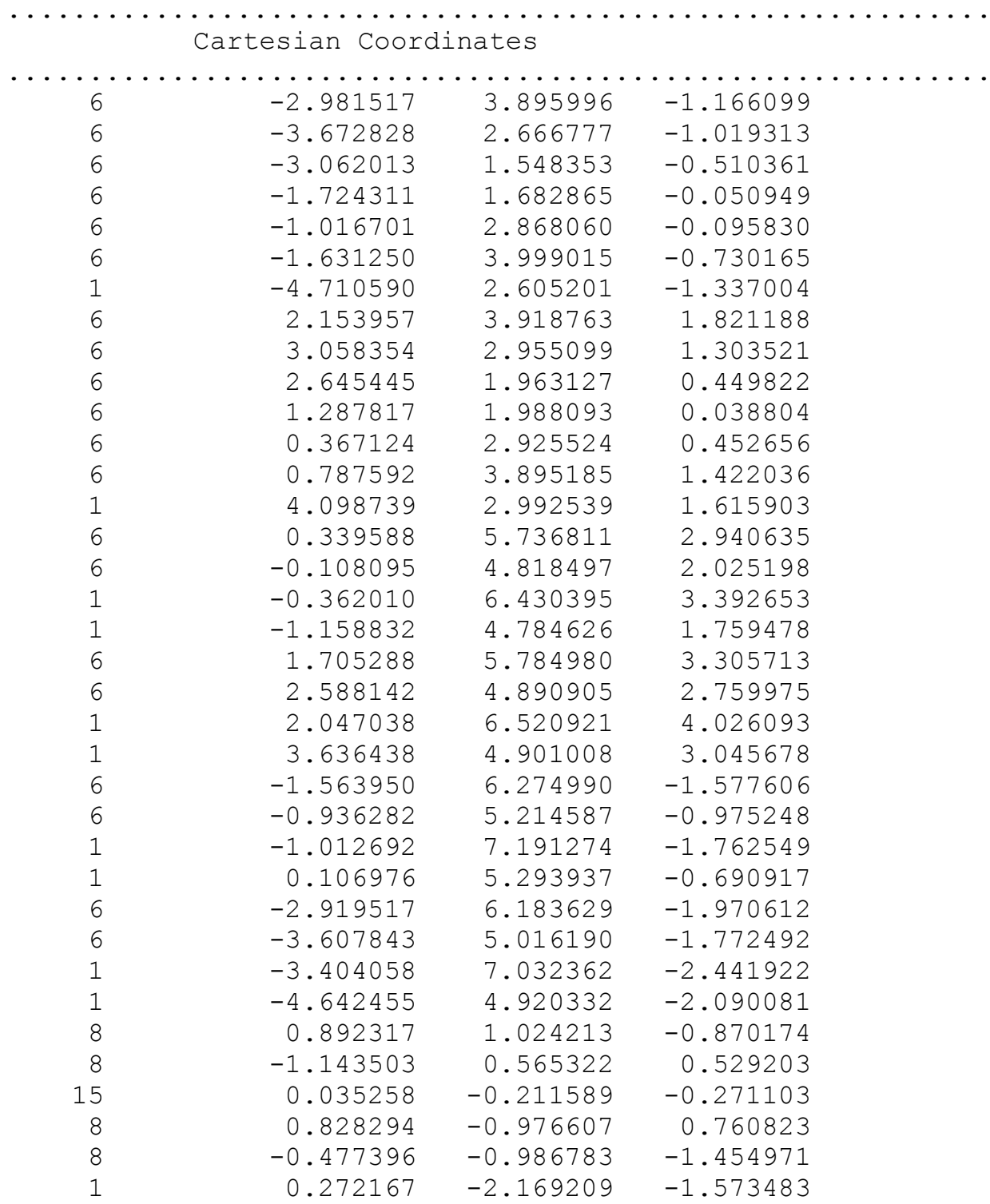




\begin{tabular}{|c|c|c|c|}
\hline 6 & 3.575350 & 0.897248 & -0.037131 \\
\hline 6 & 4.104230 & -0.048217 & 0.870007 \\
\hline 6 & 3.963648 & 0.867392 & -1.389846 \\
\hline 6 & 5.073034 & -0.945520 & 0.414738 \\
\hline 6 & 4.939630 & -0.047246 & -1.792275 \\
\hline 6 & 5.533607 & -0.937639 & -0.903319 \\
\hline 6 & -3.795181 & 0.247191 & -0.408251 \\
\hline 6 & -3.881116 & -0.602684 & -1.526914 \\
\hline 6 & -4.431555 & -0.098589 & 0.797627 \\
\hline 6 & -4.645130 & -1.767406 & -1.421795 \\
\hline 6 & -5.185086 & -1.271280 & 0.851522 \\
\hline 6 & -5.313183 & -2.114342 & -0.250690 \\
\hline 8 & 0.933081 & -2.994590 & -1.560861 \\
\hline 1 & -5.679432 & -1.536234 & 1.784892 \\
\hline 1 & -4.722630 & -2.429632 & -2.280523 \\
\hline 1 & 5.272275 & -0.041847 & -2.828518 \\
\hline 1 & 5.507621 & -1.648568 & 1.123919 \\
\hline 6 & -4.290923 & 0.744166 & 2.055332 \\
\hline 1 & -3.742424 & 1.655949 & 1.799223 \\
\hline 6 & -3.198014 & -0.268303 & -2.844369 \\
\hline 1 & -2.341286 & 0.375103 & -2.620434 \\
\hline 6 & -6.147915 & -3.379553 & -0.175620 \\
\hline 1 & -6.139621 & -3.833301 & -1.174599 \\
\hline 6 & -3.465235 & -0.010162 & 3.104215 \\
\hline 1 & -2.479491 & -0.261153 & 2.702885 \\
\hline 1 & -3.330759 & 0.597861 & 4.004474 \\
\hline 1 & -3.964869 & -0.941477 & 3.395557 \\
\hline 6 & -5.651200 & 1.169777 & 2.616317 \\
\hline 1 & -6.231214 & 1.715872 & 1.867071 \\
\hline 1 & -6.240594 & 0.303060 & 2.931924 \\
\hline 1 & -5.518184 & 1.816486 & 3.488921 \\
\hline 6 & -2.657847 & -1.508350 & -3.563221 \\
\hline 1 & -3.465488 & -2.092128 & -4.018225 \\
\hline 1 & -1.985319 & -1.201196 & -4.369230 \\
\hline 1 & -2.099950 & -2.149920 & -2.878913 \\
\hline 6 & -4.153067 & 0.495169 & -3.771638 \\
\hline 1 & -3.660936 & 0.723127 & -4.722463 \\
\hline 1 & -5.040108 & -0.111569 & -3.984548 \\
\hline 1 & -4.485506 & 1.436468 & -3.326977 \\
\hline 6 & -7.603594 & -3.068470 & 0.189115 \\
\hline 1 & -7.671150 & -2.639439 & 1.19423 \\
\hline 1 & -8.039638 & -2.351216 & -0.511040 \\
\hline 1 & -8.208701 & -3.980014 & 0.174856 \\
\hline 6 & -5.546716 & -4.392718 & 0.805106 \\
\hline 1 & -6.156473 & -5.300650 & 0.84541 \\
\hline 1 & -4.530981 & -4.671629 & 0.51145 \\
\hline 1 & -5.503174 & -3.972248 & 1.816527 \\
\hline 6 & 3.712604 & -0.076717 & 2.343145 \\
\hline 1 & 2.850181 & 0.581743 & 2.477465 \\
\hline 6 & 3.396259 & 1.816185 & -2.434566 \\
\hline 1 & 2.656190 & 2.462744 & -1.956735 \\
\hline 6 & 6.706154 & -1.797672 & -1.343242 \\
\hline 1 & 6.886678 & -1.576217 & -2.402610 \\
\hline 6 & 7.970515 & -1.416432 & -0.56311 \\
\hline 1 & 8.836751 & -1.973108 & -0.933442 \\
\hline 1 & 8.179425 & -0.347488 & -0.653659 \\
\hline 1 & 7.852721 & -1.646750 & 0.500938 \\
\hline 6 & 6.434744 & -3.298686 & -1.221 \\
\hline
\end{tabular}




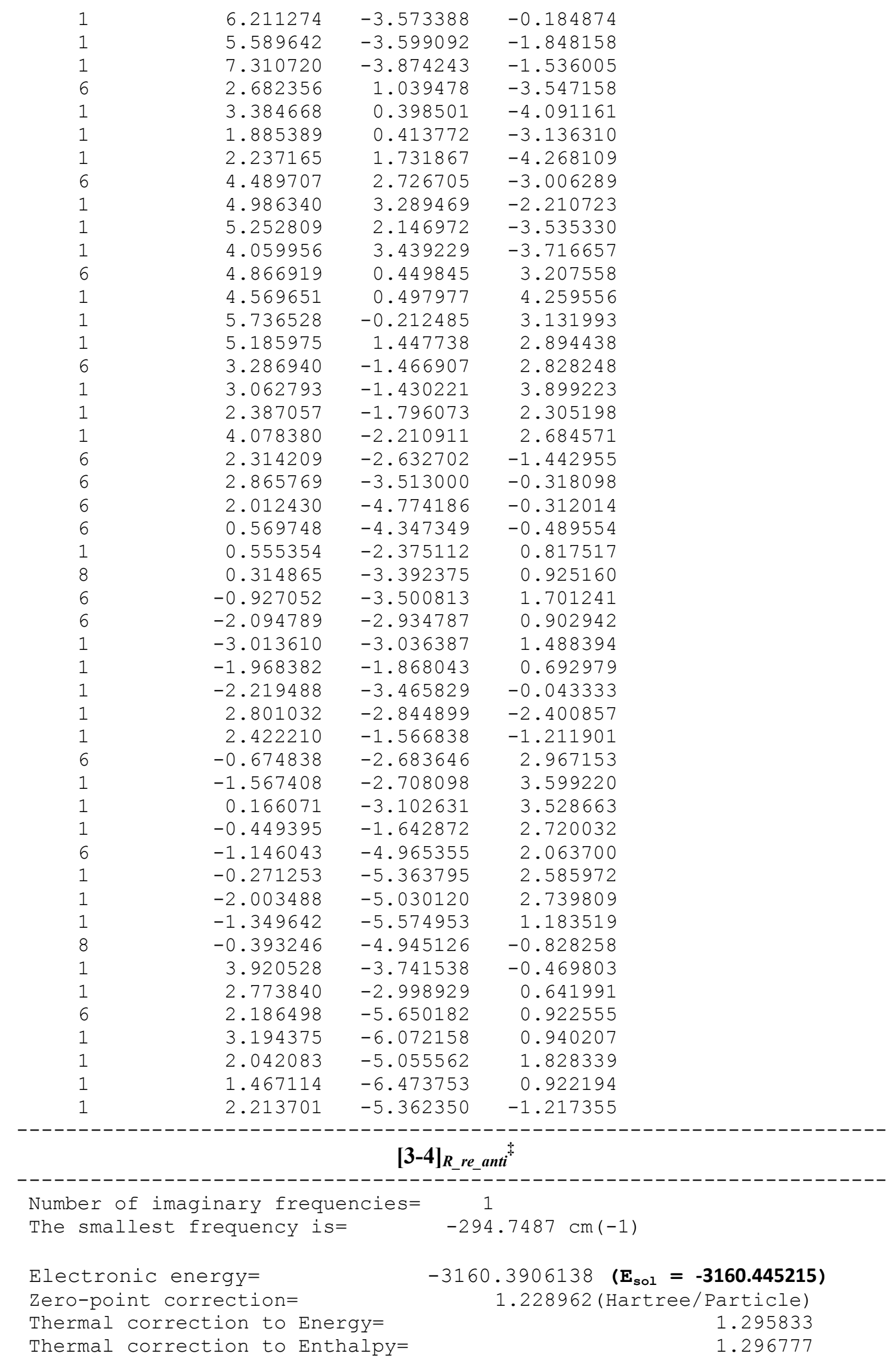




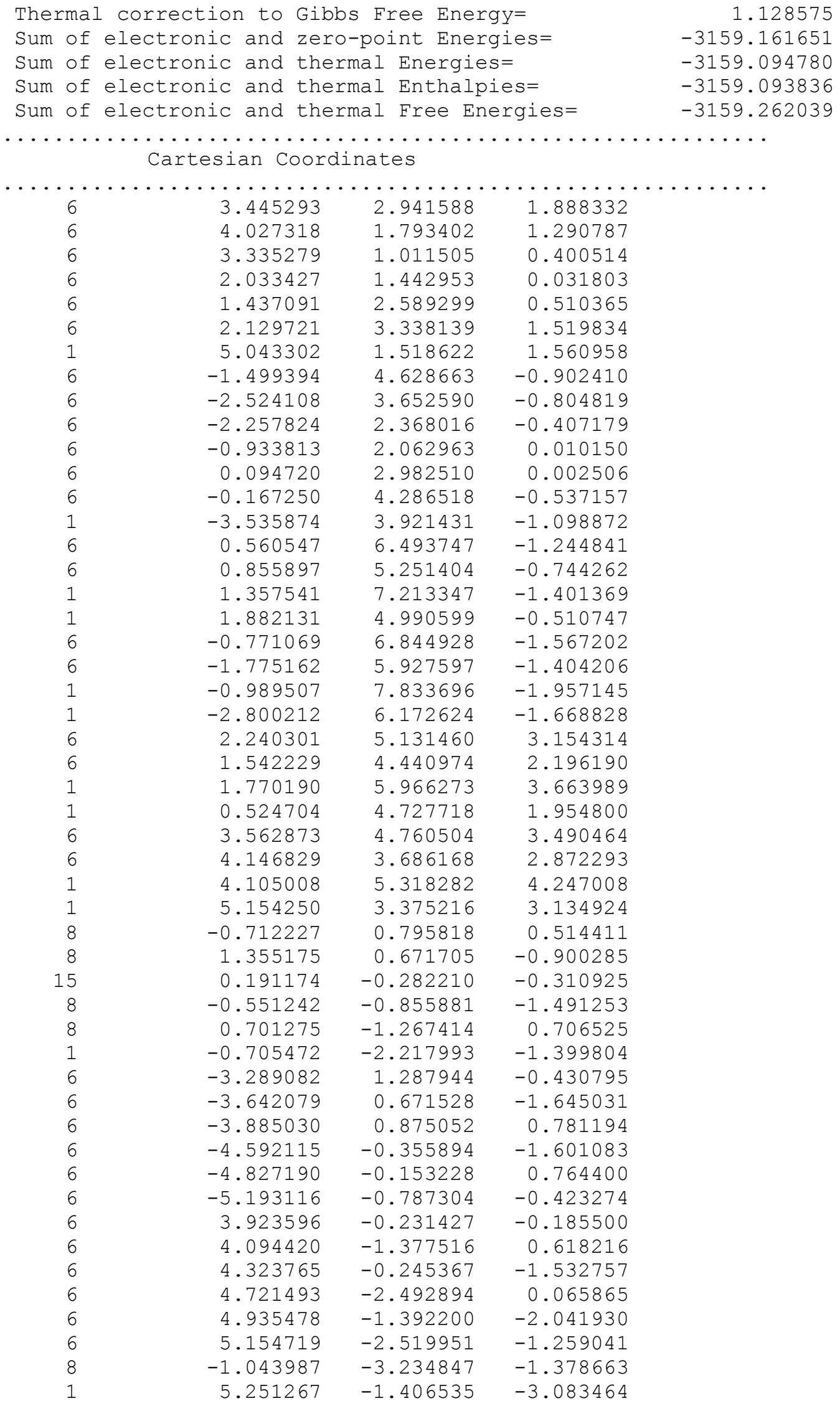




\begin{tabular}{|c|c|c|c|}
\hline 1 & 4.862985 & -3.375832 & 0.685191 \\
\hline 1 & -5.286586 & -0.462192 & 1.697952 \\
\hline 1 & -4.876949 & -0.836457 & -2.538095 \\
\hline 6 & 4.122042 & 0.943055 & -2.460115 \\
\hline 1 & 3.623846 & 1.741214 & -1.902331 \\
\hline 6 & 3.609885 & -1.440477 & 2.059801 \\
\hline 1 & 2.885078 & -0.633052 & 2.202307 \\
\hline 6 & 5.832810 & -3.749659 & -1.831734 \\
\hline 1 & 6.059400 & -3.535580 & -2.884000 \\
\hline 6 & 3.217771 & 0.572876 & -3.641649 \\
\hline 1 & 2.259399 & 0.184603 & -3.288305 \\
\hline 1 & 3.028411 & 1.452035 & -4.265568 \\
\hline 1 & 3.688773 & -0.189069 & -4.271740 \\
\hline 6 & 5.465934 & 1.500154 & -2.944366 \\
\hline 1 & 6.098022 & 1.788950 & -2.099737 \\
\hline 1 & 6.011080 & 0.755637 & -3.533623 \\
\hline 1 & 5.310483 & 2.379819 & -3.576824 \\
\hline 6 & 2.889436 & -2.758536 & 2.370077 \\
\hline 1 & 3.588187 & -3.600824 & 2.412348 \\
\hline 1 & 2.412585 & -2.691183 & 3.354783 \\
\hline 1 & 2.127275 & -2.974212 & 1.617310 \\
\hline 6 & 4.770408 & -1.239570 & 3.042781 \\
\hline 1 & 4.414654 & -1.298845 & 4.076453 \\
\hline 1 & 5.528127 & -2.017695 & 2.899408 \\
\hline 1 & 5.257571 & -0.270835 & 2.906381 \\
\hline 6 & 4.909886 & -4.972212 & -1.785518 \\
\hline 1 & 4.671398 & -5.240144 & -0.751198 \\
\hline 1 & 3.967749 & -4.774577 & -2.302761 \\
\hline 1 & 5.391484 & -5.836520 & -2.253491 \\
\hline 6 & 7.155212 & -4.039002 & -1.113032 \\
\hline 1 & 7.661765 & -4.897465 & -1.564968 \\
\hline 1 & 7.825610 & -3.176592 & -1.158184 \\
\hline 1 & 6.977645 & -4.271480 & -0.057847 \\
\hline 6 & -3.170466 & 1.058896 & -3.050696 \\
\hline 1 & -3.246920 & 0.127255 & -3.626805 \\
\hline 6 & -3.573655 & 1.608752 & 2.080606 \\
\hline 1 & -2.503087 & 1.846387 & 2.082788 \\
\hline 6 & -6.258337 & -1.868895 & -0.490262 \\
\hline 1 & -5.921528 & -2.601494 & -1.237421 \\
\hline 6 & -6.479864 & -2.608472 & 0.828034 \\
\hline 1 & -7.171266 & -3.442756 & 0.679888 \\
\hline 1 & -5.544232 & -3.005216 & 1.233736 \\
\hline 1 & -6.922692 & -1.948546 & 1.581248 \\
\hline 6 & -7.579335 & -1.266728 & -0.989879 \\
\hline 1 & -7.939604 & -0.514976 & -0.279956 \\
\hline 1 & -7.448842 & -0.777285 & -1.958633 \\
\hline 1 & -8.347932 & -2.039061 & -1.092203 \\
\hline 6 & -3.868600 & 0.801952 & 3.347277 \\
\hline 1 & -4.945766 & 0.669816 & 3.4 \\
\hline 1 & -3.402795 & -0.185969 & 3.327892 \\
\hline 1 & -3.486046 & 1.338713 & 4.219979 \\
\hline 6 & -4.360798 & 2.927312 & 2.139314 \\
\hline 1 & -4.124823 & 3.577389 & 1.29499 \\
\hline 1 & -5.435789 & 2.718490 & 2.12084 \\
\hline 1 & -4.133563 & 3.468948 & 3.06283 \\
\hline 6 & -1.739003 & 1.565149 & -3.266607 \\
\hline 1 & -1.518001 & 1.504372 & -4.33733 \\
\hline 1 & -1.613959 & 2.608869 & -2.97154 \\
\hline
\end{tabular}









\begin{tabular}{|c|c|c|c|}
\hline 1 & -4.650015 & 2.552471 & -1.559826 \\
\hline 6 & 2.145017 & 4.090994 & 1.690580 \\
\hline 6 & 3.049653 & 3.086073 & 1.257011 \\
\hline 6 & 2.644477 & 2.041548 & 0.464412 \\
\hline 6 & 1.294219 & 2.055748 & 0.031799 \\
\hline 6 & 0.372451 & 3.023981 & 0.361575 \\
\hline 6 & 0.786068 & 4.056455 & 1.266618 \\
\hline 1 & 4.085609 & 3.138881 & 1.580501 \\
\hline 6 & 0.330510 & 6.000867 & 2.646587 \\
\hline 6 & -0.110751 & 5.027742 & 1.786370 \\
\hline 1 & -0.371030 & 6.730194 & 3.038207 \\
\hline 1 & -1.156372 & 4.986045 & 1.501800 \\
\hline 6 & 1.689202 & 6.058350 & 3.035162 \\
\hline 6 & 2.572198 & 5.119948 & 2.569842 \\
\hline 1 & 2.024815 & 6.837320 & 3.711754 \\
\hline 1 & 3.614405 & 5.138019 & 2.876368 \\
\hline 6 & -1.508655 & 6.212941 & -1.961843 \\
\hline 6 & -0.898879 & 5.205189 & -1.259210 \\
\hline 1 & -0.954776 & 7.116588 & -2.194465 \\
\hline 1 & 0.132876 & 5.313459 & -0.944374 \\
\hline 6 & -2.848645 & 6.083011 & -2.394733 \\
\hline 6 & -3.539341 & 4.929349 & -2.133883 \\
\hline 1 & -3.320153 & 6.891215 & -2.944023 \\
\hline 1 & -4.561748 & 4.804094 & -2.479303 \\
\hline 8 & 0.886742 & 1.016698 & -0.798172 \\
\hline 8 & -1.167376 & 0.689918 & 0.609971 \\
\hline 15 & 0.058022 & -0.127226 & -0.023383 \\
\hline 8 & 0.823914 & -0.836861 & 1.021213 \\
\hline 8 & -0.536111 & -0.963944 & -1.204256 \\
\hline 1 & -0.014950 & -1.793700 & -1.364693 \\
\hline 6 & 3.578213 & 0.947617 & 0.049041 \\
\hline 6 & 4.082630 & 0.040482 & 1.007449 \\
\hline 6 & 3.992883 & 0.856507 & -1.292736 \\
\hline 6 & 5.056862 & -0.877959 & 0.612341 \\
\hline 6 & 4.962205 & -0.087865 & -1.638039 \\
\hline 6 & 5.530093 & -0.942541 & -0.698831 \\
\hline 6 & -3.754605 & 0.272215 & -0.405887 \\
\hline 6 & -3.839710 & -0.636350 & -1.477936 \\
\hline 6 & -4.376632 & -0.018401 & 0.821660 \\
\hline 6 & -4.599521 & -1.795515 & -1.310961 \\
\hline 6 & -5.124814 & -1.190074 & 0.938387 \\
\hline 6 & -5.261094 & -2.085138 & -0.120987 \\
\hline 8 & 1.143246 & -2.997193 & -1.306518 \\
\hline 1 & -5.610024 & -1.409662 & 1.887937 \\
\hline 1 & -4.676278 & -2.501554 & -2.134132 \\
\hline 1 & 5.305214 & -0.140332 & -2.669561 \\
\hline 1 & 5.465562 & -1.558042 & 1.356786 \\
\hline 6 & -4.255192 & 0.899690 & 2.028426 \\
\hline 1 & -3.674356 & 1.780304 & 1.735978 \\
\hline 6 & -3.152649 & -0.381934 & -2.811720 \\
\hline 1 & -2.314983 & 0.299824 & -2.630442 \\
\hline 6 & -6.075202 & -3.357853 & 0.019939 \\
\hline 1 & -6.147176 & -3.806386 & -0.978880 \\
\hline 6 & -3.499117 & 0.207379 & 3.169209 \\
\hline 1 & -2.493227 & -0.080203 & 2.851303 \\
\hline 1 & -3.411451 & 0.876449 & 4.031078 \\
\hline 1 & -4.024475 & -0.696625 & 3.496536 \\
\hline 6 & -5.629240 & 1.394329 & 2.494047 \\
\hline
\end{tabular}




\begin{tabular}{|c|c|c|c|}
\hline 1 & -6.161046 & 1.896270 & 1.680755 \\
\hline 1 & -6.252129 & 0.563224 & 2.840383 \\
\hline 1 & -5.521924 & 2.099433 & 3.323889 \\
\hline 6 & -2.583846 & -1.662179 & -3.436791 \\
\hline 1 & -3.377196 & -2.273297 & -3.880314 \\
\hline & -1.890764 & -1.402586 & -4.242494 \\
\hline & -2.053926 & -2.277405 & -2.705724 \\
\hline 6 & -4.113156 & 0.289293 & -3.803058 \\
\hline 1 & -3.621167 & 0.443823 & -4.768608 \\
\hline 1 & -4.990548 & -0.345886 & -3.966159 \\
\hline 1 & -4.461554 & 1.259181 & -3.439931 \\
\hline 6 & -7.498166 & -3.076647 & 0.51219 \\
\hline 1 & -7.489539 & -2.672513 & 1.52968 \\
\hline 1 & -8.003904 & -2.353168 & -0.132695 \\
\hline 1 & -8.087608 & -3.998263 & 0.528430 \\
\hline 6 & -5.370793 & -4.365275 & 0.93581 \\
\hline 1 & -5.947764 & -5.292586 & 1.008263 \\
\hline 1 & -4.371276 & -4.605425 & 0.562247 \\
\hline 1 & -5.263824 & -3.954376 & 1.946561 \\
\hline 6 & 3.613314 & 0.033033 & 2.456282 \\
\hline 1 & 2.695867 & 0.625549 & 2.515904 \\
\hline 6 & 3.459380 & 1.772187 & -2.384039 \\
\hline 1 & 2.711349 & 2.439485 & -1.948112 \\
\hline 6 & 6.652552 & -1.888235 & -1.084927 \\
\hline 1 & 6.862089 & -1.725510 & -2.149652 \\
\hline 6 & 7.930264 & -1.569590 & -0.29977 \\
\hline 1 & 8.756369 & -2.205868 & -0.631235 \\
\hline 1 & 8.222015 & -0.524622 & -0.432187 \\
\hline 1 & 7.781398 & -1.744157 & 0.770773 \\
\hline 6 & 6.260989 & -3.357339 & -0.902124 \\
\hline 1 & 6.001859 & -3.563252 & 0.142230 \\
\hline 1 & 5.399138 & -3.618064 & -1.523937 \\
\hline 1 & 7.090569 & -4.015586 & -1.177633 \\
\hline 6 & 2.770371 & 0.968782 & -3.492732 \\
\hline 1 & 3.481210 & 0.308368 & -4.000424 \\
\hline 1 & 1.960459 & 0.357953 & -3.085193 \\
\hline 1 & 2.347965 & 1.643170 & -4.243743 \\
\hline 6 & 4.574942 & 2.657983 & -2.952089 \\
\hline 1 & 5.050451 & 3.243698 & -2.160206 \\
\hline 1 & 5.350203 & 2.056212 & -3.437017 \\
\hline 1 & 4.171904 & 3.349285 & -3.698250 \\
\hline 6 & 4.668102 & 0.663938 & 3.374932 \\
\hline 1 & 4.319348 & 0.670411 & 4.412079 \\
\hline 1 & 5.600937 & 0.090776 & 3.335214 \\
\hline 1 & 4.898547 & 1.692874 & 3.08624 \\
\hline 6 & 3.270236 & -1.377861 & 2.949680 \\
\hline 1 & 2.844583 & -1.322352 & 3.956310 \\
\hline 1 & 2.533512 & -1.853349 & 2.299499 \\
\hline 1 & 4.159227 & -2.015292 & 3.007012 \\
\hline 6 & 2.510543 & -2.905559 & -0.84561 \\
\hline 6 & 2.743470 & -4.178142 & -0.016141 \\
\hline 6 & 1.681418 & -5.153181 & -0.534653 \\
\hline 6 & 0.612471 & -4.240928 & -1.097130 \\
\hline 1 & 0.506990 & -2.564684 & 1.392180 \\
\hline 8 & 0.299907 & -3.514772 & 1.45479 \\
\hline 6 & -0.995627 & -3.633005 & 2.04438 \\
\hline 6 & -2.010812 & -2.796195 & 1.267424 \\
\hline 1 & -3.011521 & -2.909251 & 1.69435 \\
\hline
\end{tabular}




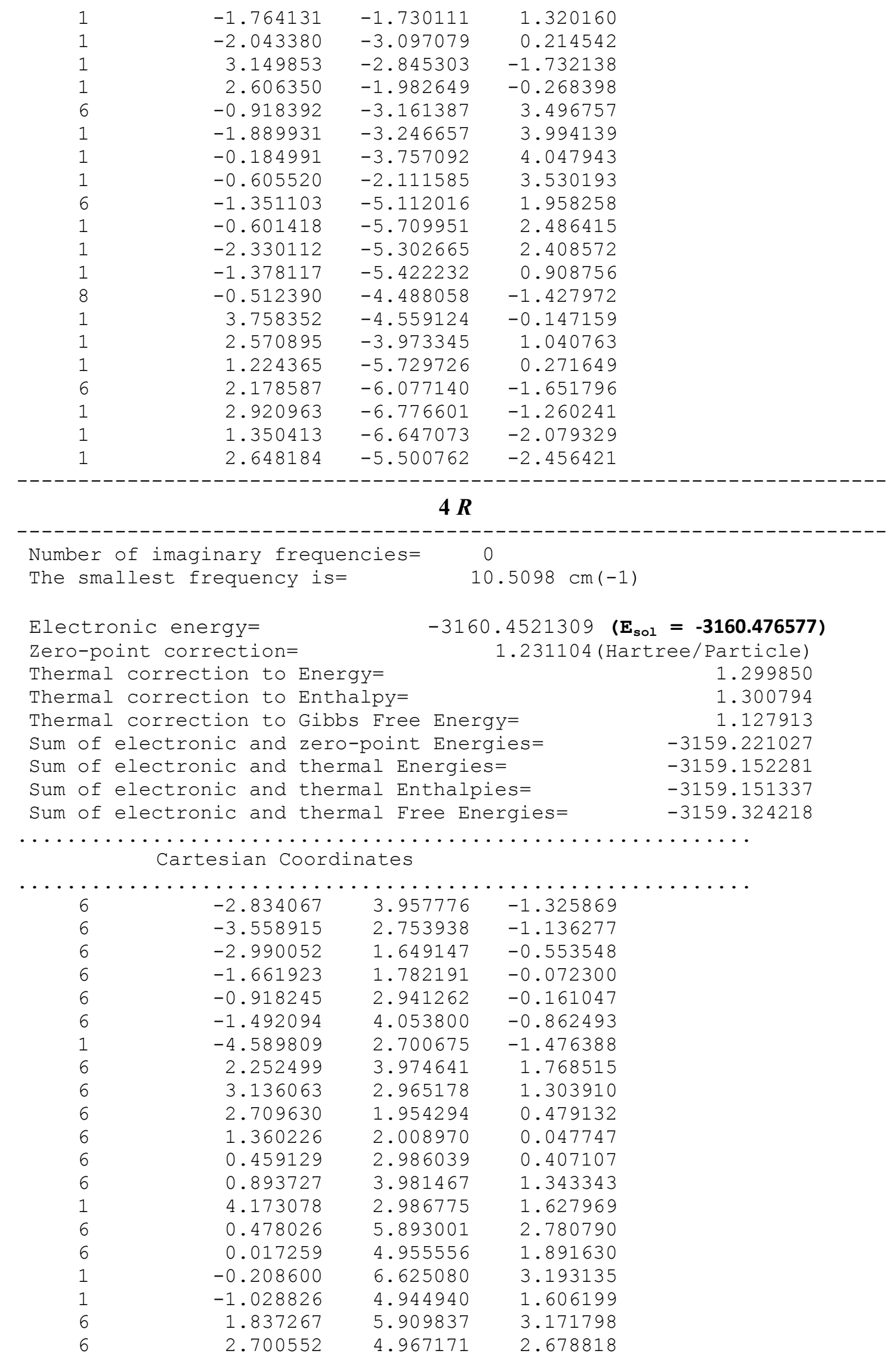




\begin{tabular}{|c|c|c|c|}
\hline 1 & 2.188941 & 6.660549 & 3.871767 \\
\hline 1 & 3.742556 & 4.953837 & 2.986221 \\
\hline 6 & -1.354369 & 6.284945 & -1.812808 \\
\hline 6 & -0.764523 & 5.241388 & -1.146267 \\
\hline 1 & -0.779251 & 7.180094 & -2.026063 \\
\hline 1 & 0.272881 & 5.312769 & -0.839250 \\
\hline 6 & -2.701937 & 6.203091 & -2.234569 \\
\hline 6 & -3.420538 & 5.061141 & -1.999503 \\
\hline 1 & -3.157135 & 7.039078 & -2.755271 \\
\hline 1 & -4.449458 & 4.972793 & -2.336903 \\
\hline 8 & 0.931943 & 1.004817 & -0.814202 \\
\hline 8 & -1.129785 & 0.676482 & 0.582535 \\
\hline 15 & 0.076436 & -0.146364 & -0.080972 \\
\hline 8 & 0.819842 & -0.916519 & 0.936452 \\
\hline 8 & -0.532672 & -0.925011 & -1.294301 \\
\hline 1 & -0.049465 & -1.776159 & -1.460257 \\
\hline 6 & 3.624739 & 0.858205 & 0.030517 \\
\hline 6 & 4.117777 & -0.082115 & 0.962374 \\
\hline 6 & 4.039084 & 0.800937 & -1.313441 \\
\hline 6 & 5.085540 & -0.996730 & 0.542247 \\
\hline 6 & 5.002884 & -0.139188 & -1.683816 \\
\hline 6 & 5.564414 & -1.023424 & -0.768227 \\
\hline 6 & -3.754642 & 0.370661 & -0.402171 \\
\hline 6 & -3.887126 & -0.504416 & -1.496550 \\
\hline 6 & -4.384196 & 0.077108 & 0.821022 \\
\hline 6 & -4.698750 & -1.631539 & -1.355330 \\
\hline 6 & -5.188055 & -1.059337 & 0.910605 \\
\hline 6 & -5.370429 & -1.919341 & -0.170652 \\
\hline 8 & 1.107321 & -2.991211 & -1.427727 \\
\hline 1 & -5.678236 & -1.281396 & 1.856877 \\
\hline 1 & -4.810317 & -2.312087 & -2.195813 \\
\hline 1 & 5.347591 & -0.164083 & -2.715775 \\
\hline 1 & 5.488152 & -1.700685 & 1.267788 \\
\hline 6 & -4.197179 & 0.944222 & 2.055981 \\
\hline 1 & -3.595054 & 1.815513 & 1.778897 \\
\hline 6 & -3.194061 & -0.245627 & -2.826240 \\
\hline 1 & -2.331392 & 0.400184 & -2.631848 \\
\hline 6 & -6.242952 & -3.155592 & -0.056832 \\
\hline 1 & -6.338894 & -3.576974 & -1.065391 \\
\hline 6 & -3.427904 & 0.175179 & 3.137088 \\
\hline 1 & -2.455104 & -0.151733 & 2.759925 \\
\hline 1 & -3.265628 & 0.805226 & 4.017334 \\
\hline 1 & -3.985766 & -0.712810 & 3.454790 \\
\hline 6 & -5.535118 & 1.465986 & 2.590662 \\
\hline 1 & -6.075717 & 2.022319 & 1.819700 \\
\hline 1 & -6.176665 & 0.643998 & 2.924380 \\
\hline 1 & -5.372168 & 2.128967 & 3.445783 \\
\hline 6 & -2.673099 & -1.531330 & -3.480165 \\
\hline 1 & -3.488973 & -2.108093 & -3.928747 \\
\hline 1 & -1.976843 & -1.279856 & -4.285647 \\
\hline 1 & -2.157853 & -2.175889 & -2.764038 \\
\hline 6 & -4.129624 & 0.484527 & -3.799127 \\
\hline 1 & -3.634484 & 0.640267 & -4.762854 \\
\hline 1 & -5.033237 & -0.109719 & -3.973054 \\
\hline 1 & -4.435805 & 1.459850 & -3.412969 \\
\hline 6 & -7.649556 & -2.819507 & 0.447662 \\
\hline 1 & -7.619384 & -2.439606 & 1.474049 \\
\hline 1 & -8.122761 & -2.058585 & -0.178515 \\
\hline
\end{tabular}




\begin{tabular}{|c|c|c|c|}
\hline 1 & -8.281238 & -3.712869 & 0.445172 \\
\hline 6 & -5.583267 & -4.216007 & 0.832600 \\
\hline 1 & -6.207195 & -5.113214 & 0.893411 \\
\hline 1 & -4.601583 & -4.501424 & 0.443930 \\
\hline & -5.444946 & -3.830868 & 1.849735 \\
\hline 6 & 3.643117 & -0.130724 & 2.408679 \\
\hline 1 & 2.742625 & 0.485086 & 2.488533 \\
\hline 6 & 3.508384 & 1.744343 & -2.381951 \\
\hline 1 & 2.769658 & 2.410040 & -1.928374 \\
\hline 6 & 6.689797 & -1.955498 & -1.178116 \\
\hline 1 & 6.898159 & -1.765414 & -2.238501 \\
\hline 6 & 7.966210 & -1.650076 & -0.38560 \\
\hline 1 & 8.794596 & -2.276261 & -0.730339 \\
\hline 1 & 8.254574 & -0.601482 & -0.494746 \\
\hline 1 & 7.816968 & -1.848378 & 0.680754 \\
\hline 6 & 6.305100 & -3.430063 & -1.032288 \\
\hline 1 & 6.057070 & -3.665249 & 0.00854 \\
\hline 1 & 5.438332 & -3.674973 & -1.653595 \\
\hline 1 & 7.134136 & -4.077702 & -1.333181 \\
\hline 6 & 2.804416 & 0.965508 & -3.499191 \\
\hline 1 & 3.508554 & 0.314185 & -4.027781 \\
\hline 1 & 1.998476 & 0.347414 & -3.09444 \\
\hline 1 & 2.374694 & 1.655328 & -4.231750 \\
\hline 6 & 4.627261 & 2.630314 & -2.942683 \\
\hline 1 & 5.114533 & 3.197089 & -2.144254 \\
\hline 1 & 5.393027 & 2.031145 & -3.445476 \\
\hline 1 & 4.224140 & 3.338652 & -3.672634 \\
\hline 6 & 4.710456 & 0.436108 & 3.354121 \\
\hline 1 & 4.357113 & 0.412434 & 4.389418 \\
\hline 1 & 5.628510 & -0.158940 & 3.296608 \\
\hline 1 & 4.968412 & 1.468938 & 3.105382 \\
\hline 6 & 3.258753 & -1.549824 & 2.845441 \\
\hline 1 & 2.844335 & -1.523979 & 3.85797 \\
\hline 1 & 2.500527 & -1.971603 & 2.183162 \\
\hline 1 & 4.127059 & -2.217295 & 2.865300 \\
\hline 6 & 2.485352 & -2.878304 & -0.993813 \\
\hline 6 & 2.743278 & -4.157818 & -0.194624 \\
\hline 6 & 1.782486 & -5.159471 & -0.838923 \\
\hline 6 & 0.641296 & -4.273376 & -1.297121 \\
\hline 1 & 0.450610 & -2.675032 & 1.240204 \\
\hline 8 & 0.181980 & -3.608388 & 1.31294 \\
\hline 6 & -1.130192 & -3.621665 & 1.88079 \\
\hline 6 & -2.092803 & -2.808499 & 1.016785 \\
\hline 1 & -3.115022 & -2.890767 & 1.397213 \\
\hline 1 & -1.841821 & -1.742834 & 1.02841 \\
\hline 1 & -2.072887 & -3.165276 & -0.01837 \\
\hline 1 & 3.110248 & -2.807551 & -1.890685 \\
\hline 1 & 2.577870 & -1.960109 & -0.409705 \\
\hline 6 & -1.058559 & -3.042862 & 3.293849 \\
\hline 1 & -2.046820 & -3.021298 & 3.76450 \\
\hline 1 & -0.384185 & -3.641180 & 3.913453 \\
\hline 1 & -0.673249 & -2.017629 & 3.254781 \\
\hline 6 & -1.563736 & -5.081972 & 1.900038 \\
\hline 1 & -0.875157 & -5.678128 & 2.507264 \\
\hline 1 & -2.569318 & -5.178552 & 2.320958 \\
\hline 1 & -1.567659 & -5.476882 & 0.879168 \\
\hline 8 & -0.484769 & -4.553382 & -1.590671 \\
\hline 1 & 3.788185 & -4.465760 & -0.2468 \\
\hline
\end{tabular}




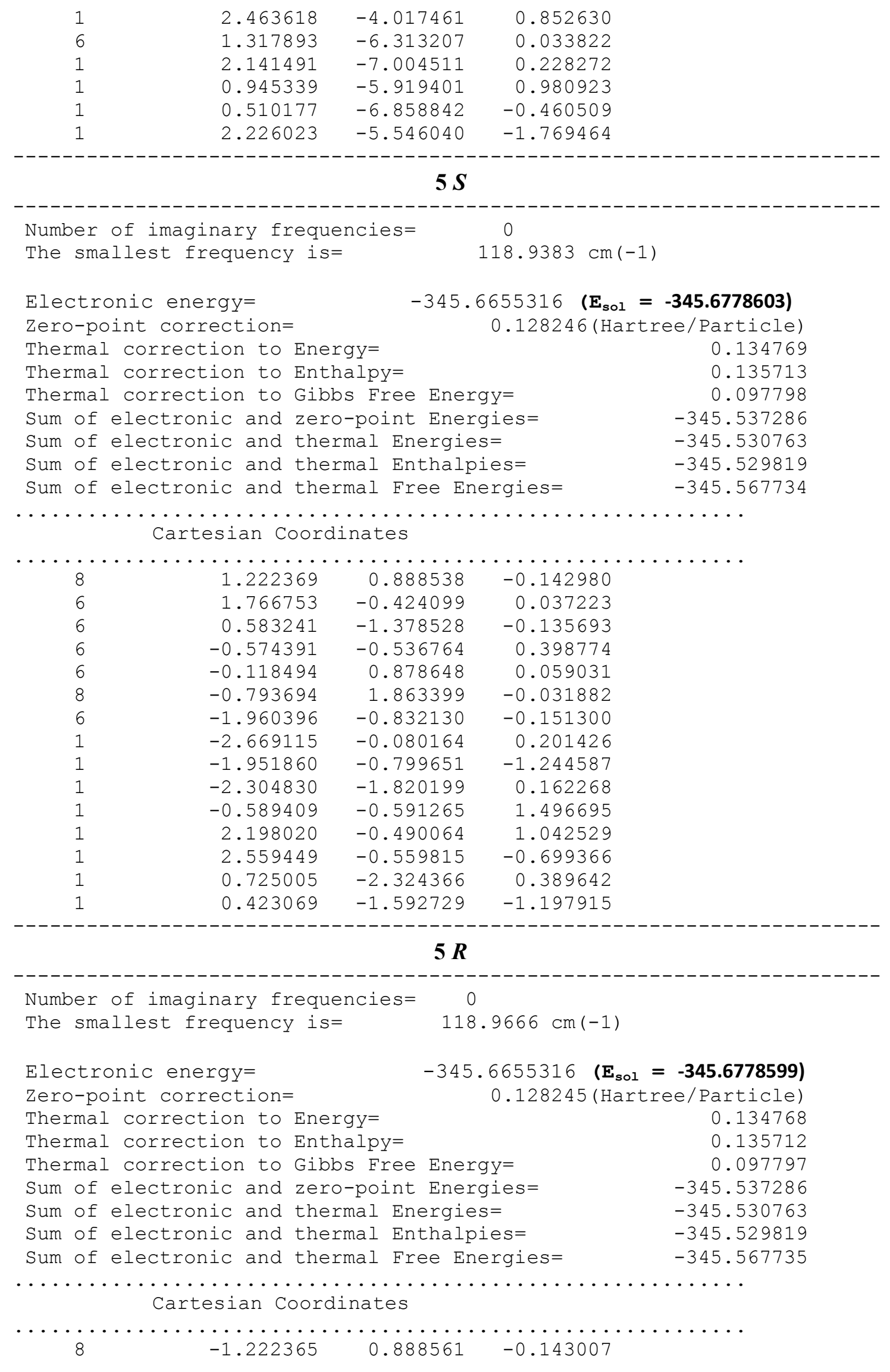




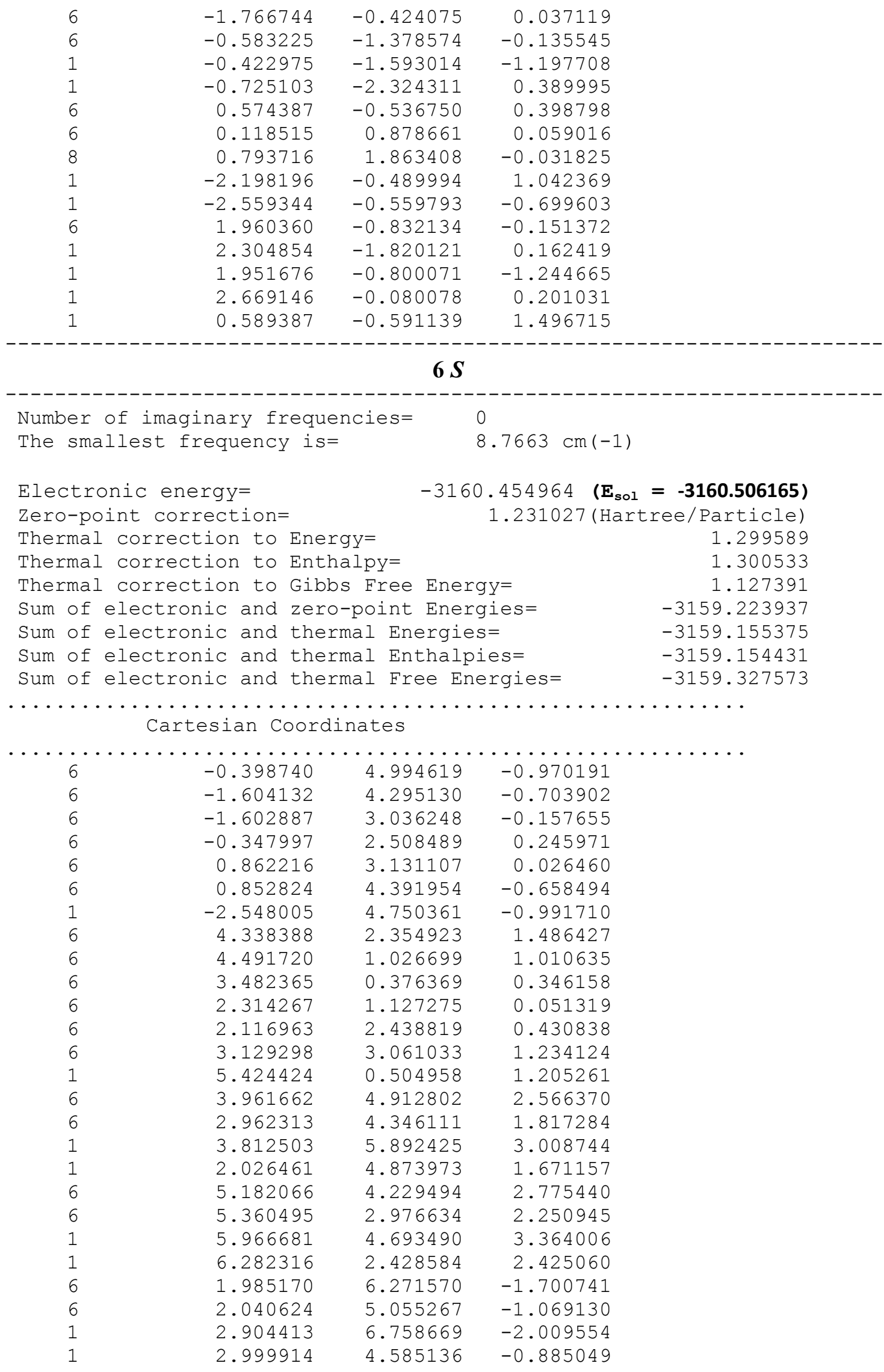




\begin{tabular}{|c|c|c|c|}
\hline 6 & 0.741982 & 6.892910 & -1.962240 \\
\hline 6 & -0.421702 & 6.261380 & -1.611020 \\
\hline 1 & 0.713312 & 7.858310 & -2.456793 \\
\hline 1 & -1.386022 & 6.711444 & -1.830466 \\
\hline 8 & 1.334527 & 0.485970 & -0.701337 \\
\hline 8 & -0.369378 & 1.301078 & 0.927970 \\
\hline 15 & 0.064381 & -0.034618 & 0.141121 \\
\hline 8 & 0.304018 & -1.125042 & 1.109403 \\
\hline 8 & -0.960175 & -0.301281 & -1.006598 \\
\hline 1 & 0.095002 & -2.842361 & 0.651415 \\
\hline 6 & 3.577817 & -1.062536 & -0.053615 \\
\hline 6 & 3.450475 & -2.070847 & 0.926845 \\
\hline 6 & 3.770443 & -1.406884 & -1.401403 \\
\hline 6 & 3.569821 & -3.400970 & 0.529097 \\
\hline 6 & 3.895326 & -2.754900 & -1.747812 \\
\hline 6 & 3.801023 & -3.766485 & -0.797276 \\
\hline 6 & -2.828418 & 2.183680 & -0.088557 \\
\hline 6 & -3.395282 & 1.706411 & -1.292869 \\
\hline 6 & -3.379309 & 1.806430 & 1.147798 \\
\hline 6 & -4.520996 & 0.887844 & -1.223778 \\
\hline 6 & -4.510155 & 0.987216 & 1.160695 \\
\hline 6 & -5.091448 & 0.511328 & -0.009285 \\
\hline 8 & -0.099802 & -3.771161 & 0.421698 \\
\hline 1 & -4.944781 & 0.698430 & 2.116132 \\
\hline 1 & -4.961158 & 0.521478 & -2.148235 \\
\hline 1 & 4.050441 & -3.011326 & -2.792534 \\
\hline 1 & 3.452009 & -4.187719 & 1.270810 \\
\hline 6 & -2.803682 & 2.267977 & 2.476665 \\
\hline 1 & -1.885015 & 2.828870 & 2.280662 \\
\hline 6 & -2.826162 & 2.040194 & -2.666612 \\
\hline 1 & -1.816248 & 2.437607 & -2.532560 \\
\hline 6 & -6.306846 & -0.393040 & 0.034079 \\
\hline 1 & -6.451769 & -0.690923 & 1.081089 \\
\hline 6 & -2.437907 & 1.078285 & 3.372052 \\
\hline 1 & -1.737255 & 0.408774 & 2.866251 \\
\hline 1 & -1.971604 & 1.430462 & 4.297406 \\
\hline 1 & -3.327805 & 0.501548 & 3.648173 \\
\hline 6 & -3.777170 & 3.215343 & 3.188475 \\
\hline 1 & -4.020169 & 4.076168 & 2.559038 \\
\hline 1 & -4.713244 & 2.702401 & 3.432979 \\
\hline 1 & -3.341713 & 3.582442 & 4.122907 \\
\hline 6 & -2.705801 & 0.812020 & -3.576686 \\
\hline 1 & -3.686535 & 0.416565 & -3.860608 \\
\hline 1 & -2.190024 & 1.089238 & -4.501080 \\
\hline 1 & -2.133470 & 0.019814 & -3.089699 \\
\hline 6 & -3.679158 & 3.114373 & -3.354738 \\
\hline 1 & -3.265011 & 3.367074 & -4.335777 \\
\hline 1 & -4.701764 & 2.749509 & -3.500485 \\
\hline 1 & -3.736221 & 4.029840 & -2.760040 \\
\hline 6 & -6.091857 & -1.664450 & -0.793344 \\
\hline 1 & -6.055531 & -1.435669 & -1.864085 \\
\hline 1 & -5.148134 & -2.146582 & -0.523356 \\
\hline 1 & -6.910423 & -2.373895 & -0.634712 \\
\hline 6 & -7.563781 & 0.352735 & -0.427895 \\
\hline 1 & -8.444345 & -0.294937 & -0.372766 \\
\hline 1 & -7.745287 & 1.238231 & 0.187032 \\
\hline 1 & -7.450175 & 0.682522 & -1.466148 \\
\hline r & 3.177840 & -1.753932 & 2.391084 \\
\hline
\end{tabular}




\begin{tabular}{|c|c|c|c|}
\hline 1 & 2.701830 & -0.769131 & 2.436256 \\
\hline 6 & 3.840246 & -0.363846 & -2.505499 \\
\hline 1 & 3.687683 & 0.623437 & -2.059070 \\
\hline 6 & 3.943542 & -5.236623 & -1.147024 \\
\hline & 3.263861 & -5.786434 & -0.482048 \\
\hline 6 & 3.556625 & -5.566235 & -2.588645 \\
\hline 1 & 3.545832 & -6.649443 & -2.738157 \\
\hline 1 & 2.565952 & -5.173413 & -2.837907 \\
\hline 1 & 4.276554 & -5.146694 & -3.299008 \\
\hline 6 & 5.374500 & -5.707799 & -0.853976 \\
\hline 1 & 6.084066 & -5.165297 & -1.487760 \\
\hline 1 & 5.644882 & -5.520371 & 0.188655 \\
\hline 1 & 5.483989 & -6.778263 & -1.054037 \\
\hline 6 & 2.728576 & -0.579966 & -3.539244 \\
\hline 1 & 2.851899 & -1.538736 & -4.053994 \\
\hline 1 & 1.745870 & -0.568761 & -3.060015 \\
\hline 1 & 2.754184 & 0.210215 & -4.296010 \\
\hline 6 & 5.220844 & -0.355912 & -3.171802 \\
\hline 1 & 6.009914 & -0.175188 & -2.436389 \\
\hline 1 & 5.427514 & -1.314961 & -3.658208 \\
\hline 1 & 5.274639 & 0.425568 & -3.935977 \\
\hline 6 & 4.485591 & -1.704980 & 3.193556 \\
\hline 1 & 4.278521 & -1.501257 & 4.248819 \\
\hline 1 & 5.001335 & -2.669229 & 3.127620 \\
\hline 1 & 5.166915 & -0.934539 & 2.826151 \\
\hline 6 & 2.221013 & -2.758299 & 3.045223 \\
\hline 1 & 1.851407 & -2.351966 & 3.991135 \\
\hline 1 & 1.366629 & -2.975747 & 2.403054 \\
\hline 1 & 2.729708 & -3.701440 & 3.273556 \\
\hline 6 & 0.253321 & -3.998617 & -0.925280 \\
\hline 6 & -0.834176 & -3.521517 & -1.891239 \\
\hline 1 & -0.660012 & -3.959869 & -2.881150 \\
\hline 1 & -0.782810 & -2.435186 & -2.020372 \\
\hline 6 & -2.242400 & -3.907790 & -1.424386 \\
\hline 6 & -2.580260 & -3.213401 & -0.120273 \\
\hline 1 & -1.672718 & -0.970601 & -0.700466 \\
\hline 8 & -2.515834 & -1.993036 & 0.026430 \\
\hline 1 & 1.210323 & -3.520108 & -1.174603 \\
\hline 1 & 0.386261 & -5.082678 & -1.033369 \\
\hline 8 & -3.060910 & -4.026634 & 0.792994 \\
\hline 6 & -3.291188 & -3.590666 & 2.17860 \\
\hline 6 & -1.983022 & -3.086832 & 2.776838 \\
\hline 1 & -1.641090 & -2.170279 & 2.292856 \\
\hline 1 & -2.140835 & -2.882775 & 3.840216 \\
\hline 1 & -1.205645 & -3.847502 & 2.669929 \\
\hline 6 & -3.735740 & -4.880917 & 2.855572 \\
\hline 1 & -3.932579 & -4.692838 & 3.914075 \\
\hline 1 & -4.648909 & -5.263537 & 2.391791 \\
\hline 1 & -2.954402 & -5.640000 & 2.771985 \\
\hline 6 & -4.398450 & -2.544329 & 2.231039 \\
\hline 1 & -5.306382 & -2.927024 & 1.753592 \\
\hline 1 & -4.629209 & -2.329648 & 3.279441 \\
\hline 1 & -4.091803 & -1.618855 & 1.740059 \\
\hline 6 & -3.303605 & -3.508969 & -2.458138 \\
\hline 1 & -3.086747 & -3.978276 & -3.420860 \\
\hline 1 & -4.304021 & -3.818061 & -2.142111 \\
\hline 1 & -3.309247 & -2.422251 & -2.595526 \\
\hline 1 & -2.286910 & -4.985205 & -1.236 \\
\hline
\end{tabular}




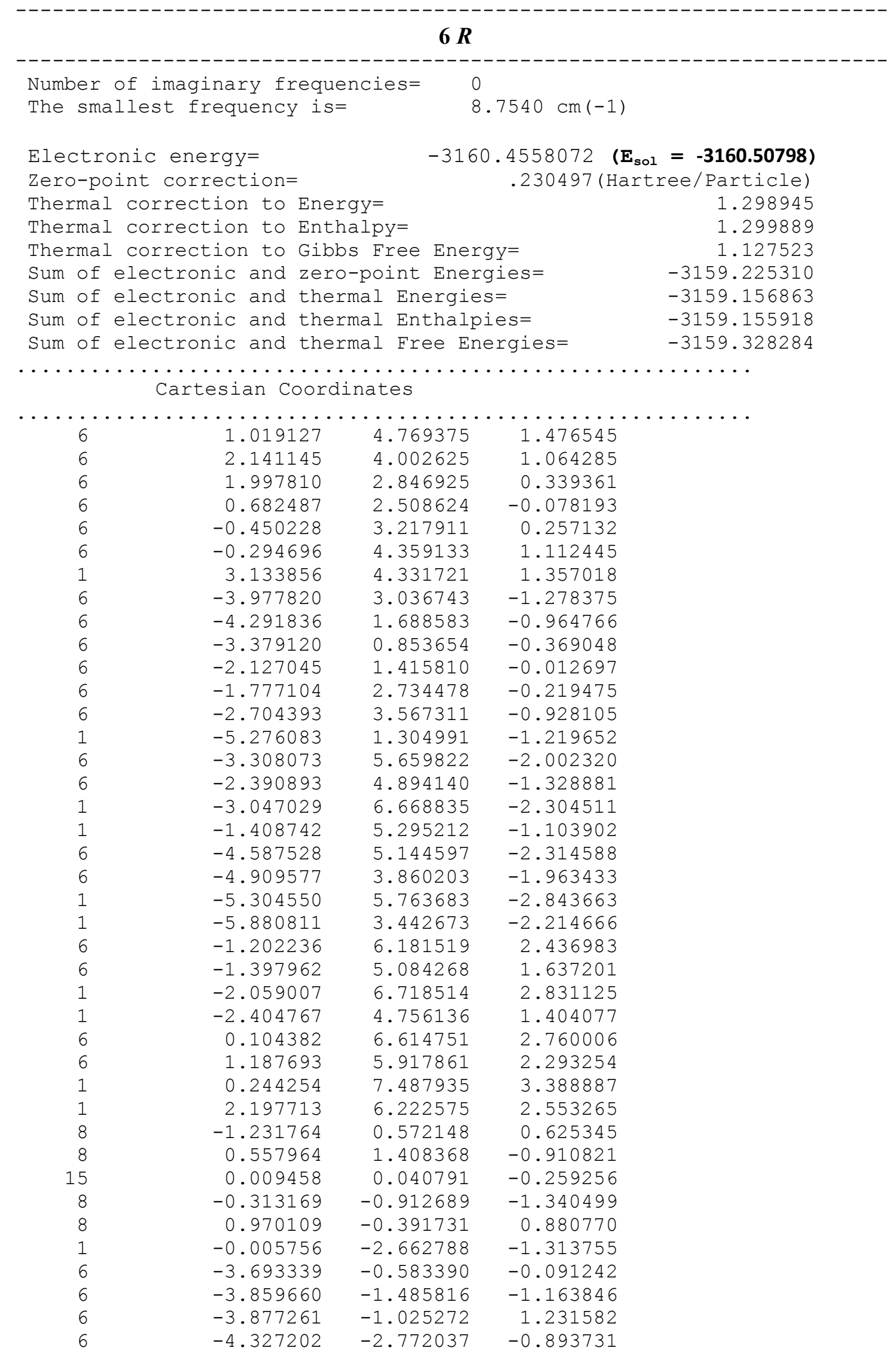




\begin{tabular}{|c|c|c|c|}
\hline 6 & -4.314348 & -2.334404 & 1.450864 \\
\hline 6 & -4.583954 & -3.211003 & 0.404958 \\
\hline 6 & 3.136738 & 1.933639 & 0.008594 \\
\hline 6 & 3.717125 & 1.141174 & 1.021911 \\
\hline 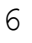 & 3.584071 & 1.818410 & -1.320482 \\
\hline 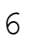 & 4.756015 & 0.274706 & 0.678358 \\
\hline 6 & 4.631722 & 0.944298 & -1.609937 \\
\hline 6 & 5.230311 & 0.161232 & -0.626416 \\
\hline 8 & -0.011612 & -3.581426 & -0.989768 \\
\hline 1 & 4.981352 & 0.870631 & -2.637312 \\
\hline 1 & 5.205628 & -0.346125 & 1.449897 \\
\hline 1 & -4.479826 & -2.671861 & 2.472500 \\
\hline 1 & -4.486038 & -3.455371 & -1.725425 \\
\hline 6 & 2.974579 & 2.622430 & -2.45878 \\
\hline 1 & 2.129391 & 3.195357 & -2.066032 \\
\hline 6 & 3.248396 & 1.197567 & 2.47077 \\
\hline 7 & 2.219499 & 1.571302 & 2.474152 \\
\hline 6 & 6.389915 & -0.762819 & -0.947394 \\
\hline 1 & 6.515729 & -1.435227 & -0.08797 \\
\hline 6 & 2.437401 & 1.708943 & -3.56649 \\
\hline 1 & 1.715215 & 0.992797 & -3.167148 \\
\hline 1 & 1.943591 & 2.304766 & -4.340314 \\
\hline 1 & 3.248808 & 1.151409 & -4.046199 \\
\hline 6 & 3.989809 & 3.627167 & -3.01719 \\
\hline 1 & 4.348245 & 4.301627 & -2.23427 \\
\hline 1 & 4.858145 & 3.110611 & -3.439761 \\
\hline 1 & 3.537296 & 4.228309 & -3.811782 \\
\hline 6 & 3.239991 & -0.174372 & 3.156579 \\
\hline 1 & 4.256215 & -0.522709 & 3.36978 \\
\hline 1 & 2.715146 & -0.102574 & 4.113850 \\
\hline 1 & 2.743034 & -0.929715 & 2.545087 \\
\hline 6 & 4.124267 & 2.156547 & 3.289535 \\
\hline 1 & 3.789667 & 2.188520 & 4.33125 \\
\hline 1 & 5.165612 & 1.816735 & 3.27638 \\
\hline 1 & 4.101293 & 3.173633 & 2.893207 \\
\hline 6 & 7.684774 & 0.042571 & -1.110963 \\
\hline 1 & 7.592606 & 0.740503 & -1.950039 \\
\hline 1 & 7.901478 & 0.625968 & -0.21217 \\
\hline 1 & 8.534157 & -0.618552 & -1.31000 \\
\hline 6 & 6.131783 & -1.626306 & -2.185063 \\
\hline 1 & 6.936532 & -2.356874 & -2.315018 \\
\hline 1 & 5.183619 & -2.164803 & -2.10316 \\
\hline 1 & 6.094620 & -1.015726 & -3.09283 \\
\hline 6 & -3.521010 & -1.113351 & -2.60079 \\
\hline 1 & -2.928467 & -0.193550 & -2.57806 \\
\hline 6 & -3.634340 & -0.135406 & 2.44059 \\
\hline 1 & -3.331340 & 0.855349 & 2.09102 \\
\hline 6 & -5.157647 & -4.590496 & 0.67314 \\
\hline 1 & -5.299696 & -4.676292 & 1.758079 \\
\hline 6 & -6.528771 & -4.750242 & 0.005959 \\
\hline 1 & -6.971515 & -5.718286 & 0.25954 \\
\hline 1 & -7.214858 & -3.960603 & 0.32304 \\
\hline 1 & -6.437820 & -4.698871 & -1.08396 \\
\hline 6 & -4.211068 & -5.711647 & 0.233253 \\
\hline 1 & -4.006145 & -5.650443 & -0.841121 \\
\hline 1 & -3.255074 & -5.655706 & 0.76171 \\
\hline 1 & -4.656156 & -6.691300 & 0.43336 \\
\hline 6 & -2.491998 & -0.690807 & 3.30019 \\
\hline
\end{tabular}

S61 







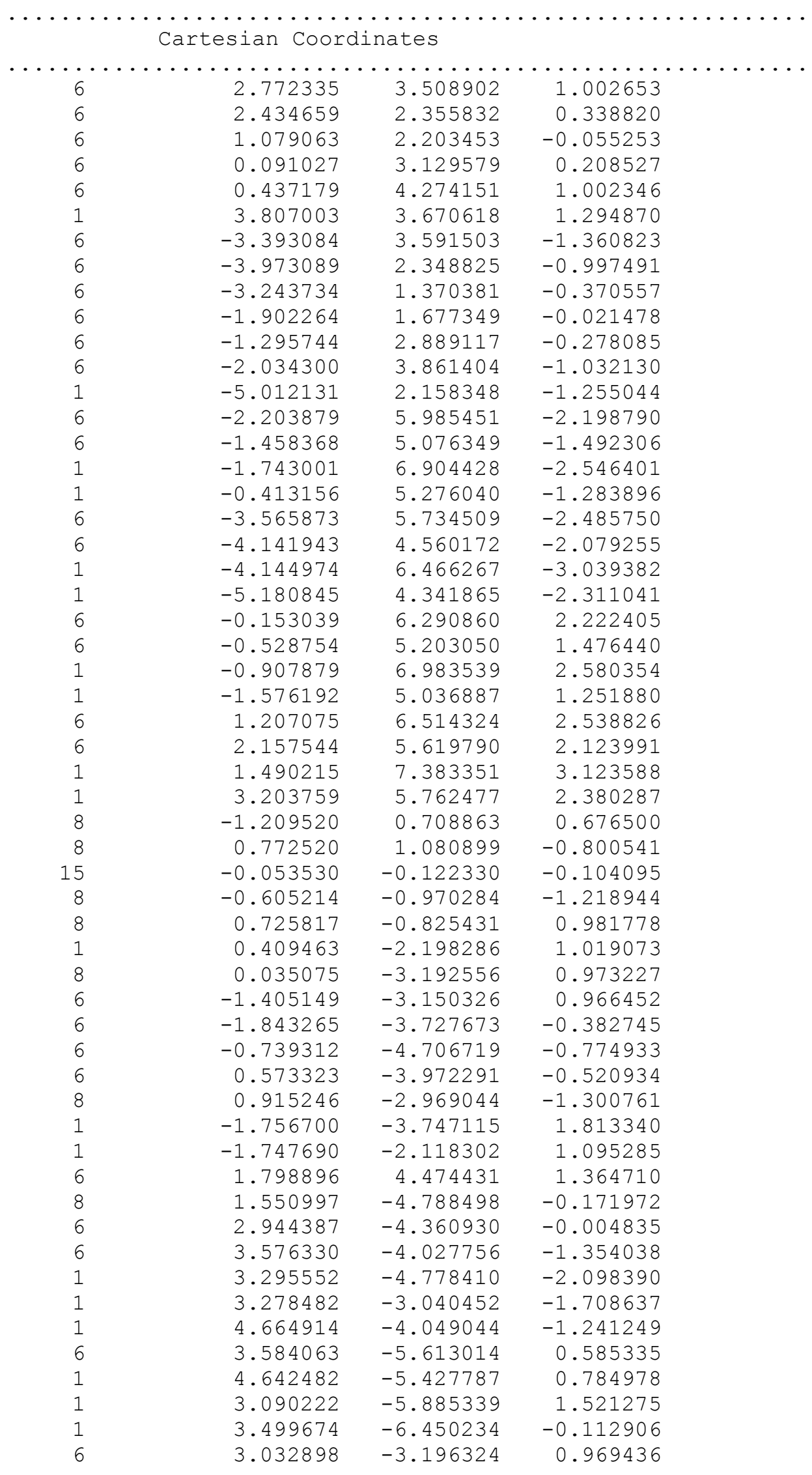




\begin{tabular}{|c|c|c|c|}
\hline L & 2.606803 & -2.278921 & 0.554871 \\
\hline 1 & 2.526052 & -3.439791 & 1.907922 \\
\hline L & 4.087457 & -2.998173 & 1.185430 \\
\hline & 0.215781 & -2.216558 & -1.366722 \\
\hline & 3.435496 & 1.279890 & 0.067935 \\
\hline 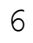 & 3.821548 & 0.977753 & -1.249329 \\
\hline 6 & 4.007817 & 0.575121 & 1.149317 \\
\hline & 4.806390 & 0.009295 & -1.465392 \\
\hline & 4.997605 & -0.367513 & 0.880918 \\
\hline 5 & 5.418966 & -0.666039 & -0.415054 \\
\hline & 5.100383 & -0.206864 & -2.48828 \\
\hline ] & 5.450637 & -0.904893 & 1.713346 \\
\hline 6 & -3.808700 & 0.019889 & -0.070475 \\
\hline 6 & -4.007840 & -0.383824 & 1.265997 \\
\hline 6 & -4.153659 & -0.846191 & -1.127394 \\
\hline 6 & -4.577007 & -1.632063 & 1.51899 \\
\hline 6 & -4.730776 & -2.080689 & -0.819633 \\
\hline 6 & -4.950977 & -2.495767 & 0.490502 \\
\hline 1 & -4.744407 & -1.929747 & 2.552142 \\
\hline & -5.009903 & -2.748908 & -1.63278 \\
\hline 6 & -5.630343 & -3.822339 & 0.777414 \\
\hline 1 & -5.623612 & -4.399320 & -0.157039 \\
\hline 6 & -3.663349 & 0.503377 & 2.453354 \\
\hline 1 & -3.205847 & 1.423458 & 2.080281 \\
\hline 6 & 3.237870 & 1.688418 & -2.460771 \\
\hline 1 & 2.460860 & 2.377981 & -2.120059 \\
\hline 6 & 3.586733 & 0.792150 & 2.596780 \\
\hline 1 & 2.720403 & 1.458601 & 2.605855 \\
\hline 6 & 6.529663 & -1.684046 & -0.618213 \\
\hline 1 & 6.251977 & -2.581680 & -0.044398 \\
\hline 6 & -3.943649 & -0.489562 & -2.593377 \\
\hline 1 & -3.379610 & 0.445210 & -2.641313 \\
\hline 6 & -5.292078 & -0.268229 & -3.291018 \\
\hline 1 & -5.885556 & 0.499152 & -2.785394 \\
\hline 1 & -5.140431 & 0.042870 & -4.329121 \\
\hline 1 & -5.882515 & -1.190934 & -3.298914 \\
\hline 6 & -3.118246 & -1.545101 & -3.337532 \\
\hline 1 & -3.598803 & -2.529264 & -3.309119 \\
\hline 1 & -3.013995 & -1.260440 & -4.38927 \\
\hline 1 & -2.119522 & -1.620996 & -2.902572 \\
\hline 6 & -4.909518 & -4.649204 & 1.846320 \\
\hline 1 & -5.415752 & -5.608272 & 1.990417 \\
\hline 1 & -3.870620 & -4.853455 & 1.569462 \\
\hline 1 & -4.904623 & -4.132362 & 2.81108 \\
\hline 6 & -7.093114 & -3.589847 & 1.177665 \\
\hline 1 & -7.144523 & -3.010814 & 2.105856 \\
\hline 1 & -7.626253 & -3.031357 & 0.403971 \\
\hline 1 & -7.609636 & -4.540888 & 1.340172 \\
\hline 6 & 6.732682 & -2.098193 & -2.074610 \\
\hline 1 & 7.471290 & -2.902214 & -2.138392 \\
\hline 1 & 5.804214 & -2.448024 & -2.534738 \\
\hline 1 & 7.108875 & -1.257800 & -2.66803 \\
\hline 6 & 7.849067 & -1.151742 & -0.040414 \\
\hline 1 & 8.152774 & -0.248604 & -0.579527 \\
\hline 1 & 7.751708 & -0.893162 & 1.017051 \\
\hline 1 & 8.645148 & -1.895957 & -0.141003 \\
\hline 6 & -2.650981 & -0.169627 & 3.38748 \\
\hline 1 & -1.723862 & -0.404910 & 2.85794 \\
\hline
\end{tabular}




$\begin{array}{rrrr}1 & -2.405922 & 0.496028 & 4.220752 \\ 1 & -3.057974 & -1.094299 & 3.811725 \\ 6 & -4.933487 & 0.902569 & 3.215539 \\ 1 & -5.648605 & 1.399876 & 2.554080 \\ 1 & -5.427359 & 0.025046 & 3.646166 \\ 1 & -4.688747 & 1.584612 & 4.035465 \\ 6 & 4.710706 & 1.458687 & 3.399118 \\ 1 & 5.597554 & 0.816346 & 3.429095 \\ 1 & 5.010219 & 2.414043 & 2.958038 \\ 1 & 4.392270 & 1.641258 & 4.430123 \\ 6 & 3.148658 & -0.516783 & 3.263679 \\ 1 & 2.317852 & -0.962727 & 2.712768 \\ 1 & 3.970512 & -1.239705 & 3.312408 \\ 1 & 2.822555 & -0.320167 & 4.290135 \\ 6 & 4.309246 & 2.527209 & -3.168497 \\ 1 & 4.757032 & 3.250948 & -2.481475 \\ 1 & 5.111726 & 1.893423 & -3.560220 \\ 1 & 3.872436 & 3.073626 & -4.010140 \\ 6 & 2.581956 & 0.697111 & -3.428718 \\ 1 & 1.807321 & 0.114578 & -2.924064 \\ 1 & 2.120419 & 1.232996 & -4.263848 \\ 1 & 3.320550 & 0.004458 & -3.846644 \\ 1 & -0.763822 & -4.903691 & -1.852052 \\ 6 & -0.824299 & -6.016803 & 0.005137 \\ 1 & -1.800154 & -6.480847 & -0.160447 \\ 1 & -0.045058 & -6.714362 & -0.304266 \\ 1 & -0.701760 & -5.839287 & 1.077851 \\ 1 & -1.938212 & -2.919140 & -1.109793 \\ 1 & -2.813496 & -4.225310 & -0.313241 \\ ---------------------------------------------\end{array}$

\section{$[6-7]_{S \_ \text {re_syn }}{ }^{\ddagger}$ (ii)}

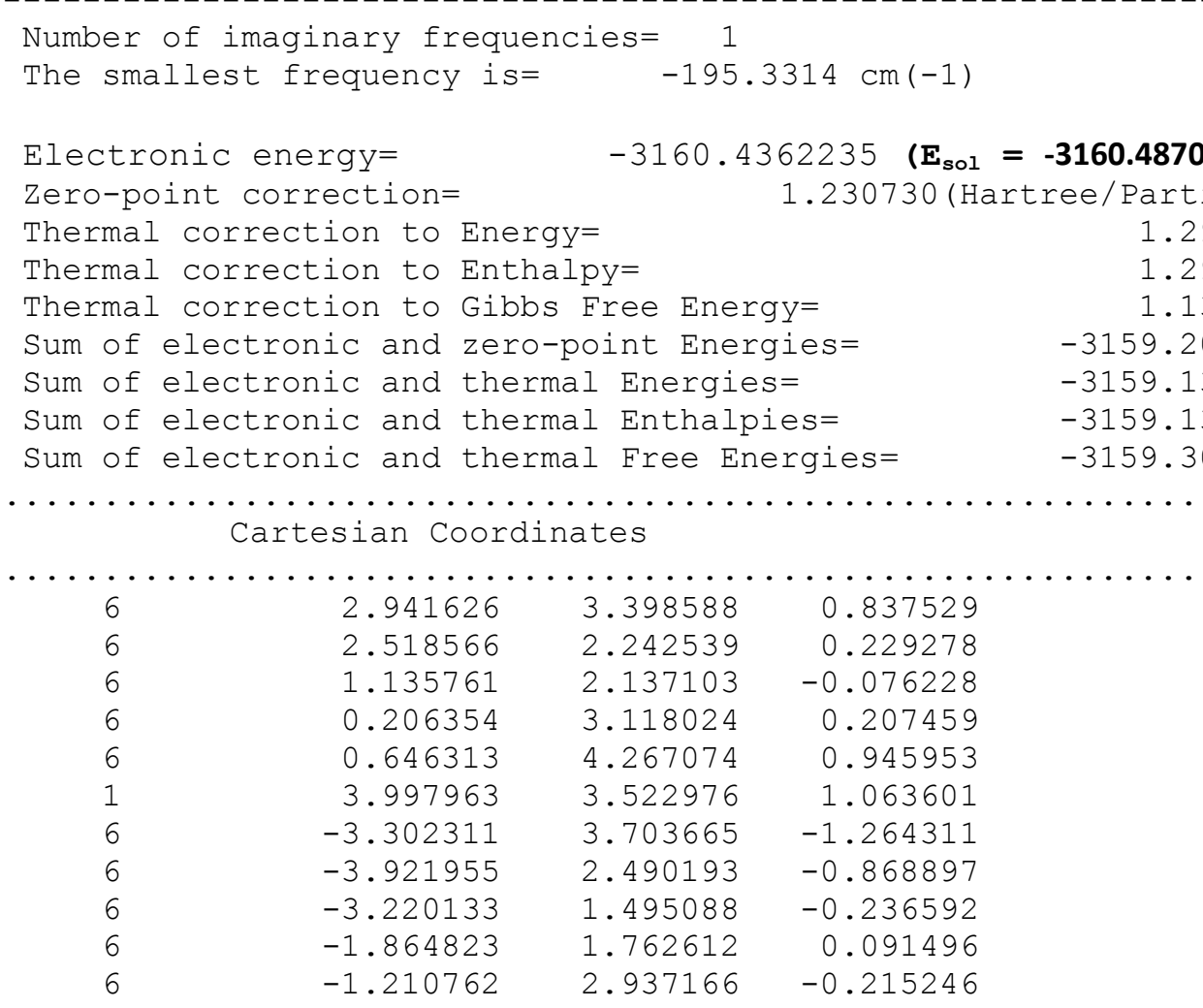




\begin{tabular}{|c|c|c|c|}
\hline 6 & -1.925854 & 3.923566 & -0.974352 \\
\hline 1 & -4.970134 & 2.332873 & -1.110488 \\
\hline 6 & -2.038176 & 6.026852 & -2.185372 \\
\hline 6 & -1.312558 & 5.102607 & -1.477702 \\
\hline 1 & -1.548439 & 6.917712 & -2.565156 \\
\hline 1 & -0.254971 & 5.263748 & -1.301795 \\
\hline 6 & -3.416188 & 5.827542 & -2.432535 \\
\hline 6 & -4.029028 & 4.686915 & -1.985833 \\
\hline 1 & -3.978720 & 6.570999 & -2.987642 \\
\hline 1 & -5.081181 & 4.505976 & -2.187942 \\
\hline 6 & 0.214270 & 6.340958 & 2.135712 \\
\hline 6 & -0.249861 & 5.252869 & 1.441840 \\
\hline 1 & -0.488435 & 7.077189 & 2.512321 \\
\hline 1 & -1.314133 & 5.129633 & 1.275376 \\
\hline 6 & 1.598282 & 6.508450 & 2.374654 \\
\hline 6 & 2.483853 & 5.560871 & 1.934818 \\
\hline 1 & 1.950868 & 7.378066 & 2.919573 \\
\hline 1 & 3.547814 & 5.661458 & 2.130942 \\
\hline 8 & -1.196017 & 0.789285 & 0.807787 \\
\hline 8 & 0.739284 & 1.003966 & -0.759139 \\
\hline 15 & -0.122585 & -0.127292 & 0.011784 \\
\hline 8 & -0.767608 & -0.974649 & -1.052119 \\
\hline 8 & 0.667342 & -0.838936 & 1.085143 \\
\hline 1 & 0.373306 & -2.202056 & 1.116227 \\
\hline 8 & 0.002661 & -3.203423 & 1.083061 \\
\hline 6 & -1.432875 & -3.169180 & 1.180214 \\
\hline 6 & -1.964467 & -3.740863 & -0.136177 \\
\hline 6 & -0.883612 & -4.710600 & -0.614029 \\
\hline 6 & 0.441526 & -3.972165 & -0.437363 \\
\hline 8 & 0.731595 & -2.969430 & -1.240398 \\
\hline 1 & -1.721397 & -3.775549 & 2.043579 \\
\hline 1 & -1.768456 & -2.139136 & 1.338752 \\
\hline 6 & 2.033377 & 4.414444 & 1.228252 \\
\hline 8 & 1.443960 & -4.786510 & -0.155844 \\
\hline 6 & 2.838162 & -4.340424 & -0.043646 \\
\hline 6 & 3.414186 & -4.010236 & -1.418031 \\
\hline 1 & 3.160660 & -4.798428 & -2.132762 \\
\hline 1 & 3.049277 & -3.053235 & -1.790641 \\
\hline 1 & 4.504921 & -3.960080 & -1.334443 \\
\hline 6 & 3.514538 & -5.579228 & 0.533078 \\
\hline 1 & 4.580881 & -5.386307 & 0.677116 \\
\hline 1 & 3.070096 & -5.839963 & 1.496803 \\
\hline 1 & 3.401147 & -6.427608 & -0.147296 \\
\hline 6 & 2.948315 & -3.166970 & 0.917780 \\
\hline 1 & 2.531082 & -2.248242 & 0.496993 \\
\hline 1 & 2.446047 & -3.395667 & 1.862157 \\
\hline 1 & 4.004585 & -2.976630 & 1.125310 \\
\hline 1 & 0.028387 & -2.218247 & -1.259353 \\
\hline 6 & 3.471399 & 1.130035 & -0.070352 \\
\hline 6 & 3.743227 & 0.754357 & -1.402652 \\
\hline 6 & 4.140964 & 0.485954 & 0.989184 \\
\hline 6 & 4.710505 & -0.219464 & -1.641103 \\
\hline 6 & 5.124997 & -0.463271 & 0.693450 \\
\hline 6 & 5.434086 & -0.824116 & -0.613709 \\
\hline 1 & 4.932186 & -0.503350 & -2.669119 \\
\hline 1 & 5.647702 & -0.939009 & 1.519786 \\
\hline 6 & -3.822605 & 0.160816 & 0.063842 \\
\hline 6 & -4.003018 & -0.257860 & 1.399270 \\
\hline
\end{tabular}




\begin{tabular}{|c|c|c|c|}
\hline 6 & -4.210441 & -0.683076 & -0.994767 \\
\hline 6 & -4.595688 & -1.494906 & 1.641446 \\
\hline 6 & -4.828445 & -1.902509 & -0.696703 \\
\hline 6 & -5.033158 & -2.328797 & 0.610713 \\
\hline 1 & -4.735274 & -1.819746 & 2.671748 \\
\hline & -5.137389 & -2.538538 & -1.521404 \\
\hline & -5.724178 & -3.637537 & 0.960651 \\
\hline & -5.085196 & -4.150214 & 1.694927 \\
\hline 6 & -3.620451 & 0.606033 & 2.592470 \\
\hline 1 & -3.143911 & 1.518202 & 2.223723 \\
\hline 6 & 3.064209 & 1.399684 & -2.601038 \\
\hline 1 & 2.333634 & 2.127826 & -2.240311 \\
\hline 6 & 3.829708 & 0.756440 & 2.456047 \\
\hline 1 & 3.001325 & 1.466877 & 2.508712 \\
\hline 6 & 6.565212 & -1.773746 & -0.969206 \\
\hline 1 & 6.241255 & -2.344358 & -1.850478 \\
\hline 6 & -3.991384 & -0.331823 & -2.460910 \\
\hline 1 & -3.372781 & 0.567829 & -2.509482 \\
\hline 6 & -5.326871 & -0.033454 & -3.154103 \\
\hline 1 & -5.873906 & 0.768527 & -2.650136 \\
\hline 1 & -5.161598 & 0.264174 & -4.194115 \\
\hline 1 & -5.968840 & -0.921142 & -3.156259 \\
\hline 6 & -3.232856 & -1.433581 & -3.210452 \\
\hline 1 & -3.790248 & -2.376615 & -3.218956 \\
\hline 1 & -3.078960 & -1.134684 & -4.252138 \\
\hline 1 & -2.255505 & -1.598549 & -2.752640 \\
\hline 6 & -7.075928 & -3.358067 & 1.633276 \\
\hline 1 & -7.559392 & -4.291566 & 1.937152 \\
\hline 1 & -6.961686 & -2.725958 & 2.517436 \\
\hline 1 & -7.739598 & -2.838887 & 0.934209 \\
\hline 6 & -5.923456 & -4.576369 & -0.228167 \\
\hline 1 & -6.629042 & -4.148863 & -0.948434 \\
\hline 1 & -4.987279 & -4.780070 & -0.756714 \\
\hline 1 & -6.337107 & -5.529428 & 0.112339 \\
\hline 6 & 7.806629 & -0.963243 & -1.370547 \\
\hline 1 & 8.622845 & -1.623761 & -1.679554 \\
\hline 1 & 7.581530 & -0.280438 & -2.194081 \\
\hline 1 & 8.151312 & -0.363499 & -0.521633 \\
\hline 6 & 6.923410 & -2.768862 & 0.133309 \\
\hline 1 & 7.350070 & -2.260250 & 1.003936 \\
\hline 1 & 6.050377 & -3.337516 & 0.468321 \\
\hline 1 & 7.672669 & -3.478446 & -0.229152 \\
\hline 6 & -2.617069 & -0.100435 & 3.510742 \\
\hline 1 & -1.697470 & -0.346465 & 2.973240 \\
\hline 1 & -2.353950 & 0.547925 & 4.352116 \\
\hline 1 & -3.040052 & -1.022538 & 3.924680 \\
\hline 6 & -4.873628 & 1.028146 & 3.370634 \\
\hline 1 & -5.582882 & 1.548228 & 2.720488 \\
\hline 1 & -5.383906 & 0.157718 & 3.796758 \\
\hline 1 & -4.605843 & 1.696357 & 4.194752 \\
\hline 6 & 5.031882 & 1.376438 & 3.177138 \\
\hline 1 & 5.886102 & 0.691075 & 3.175021 \\
\hline 1 & 5.352978 & 2.306009 & 2.697533 \\
\hline 1 & 4.782162 & 1.596482 & 4.219518 \\
\hline 6 & 3.364599 & -0.520384 & 3.166196 \\
\hline 1 & 2.473918 & -0.921918 & 2.676879 \\
\hline 1 & 4.146381 & -1.288153 & 3.162251 \\
\hline 1 & 3.120852 & -0.300599 & 4.210638 \\
\hline
\end{tabular}




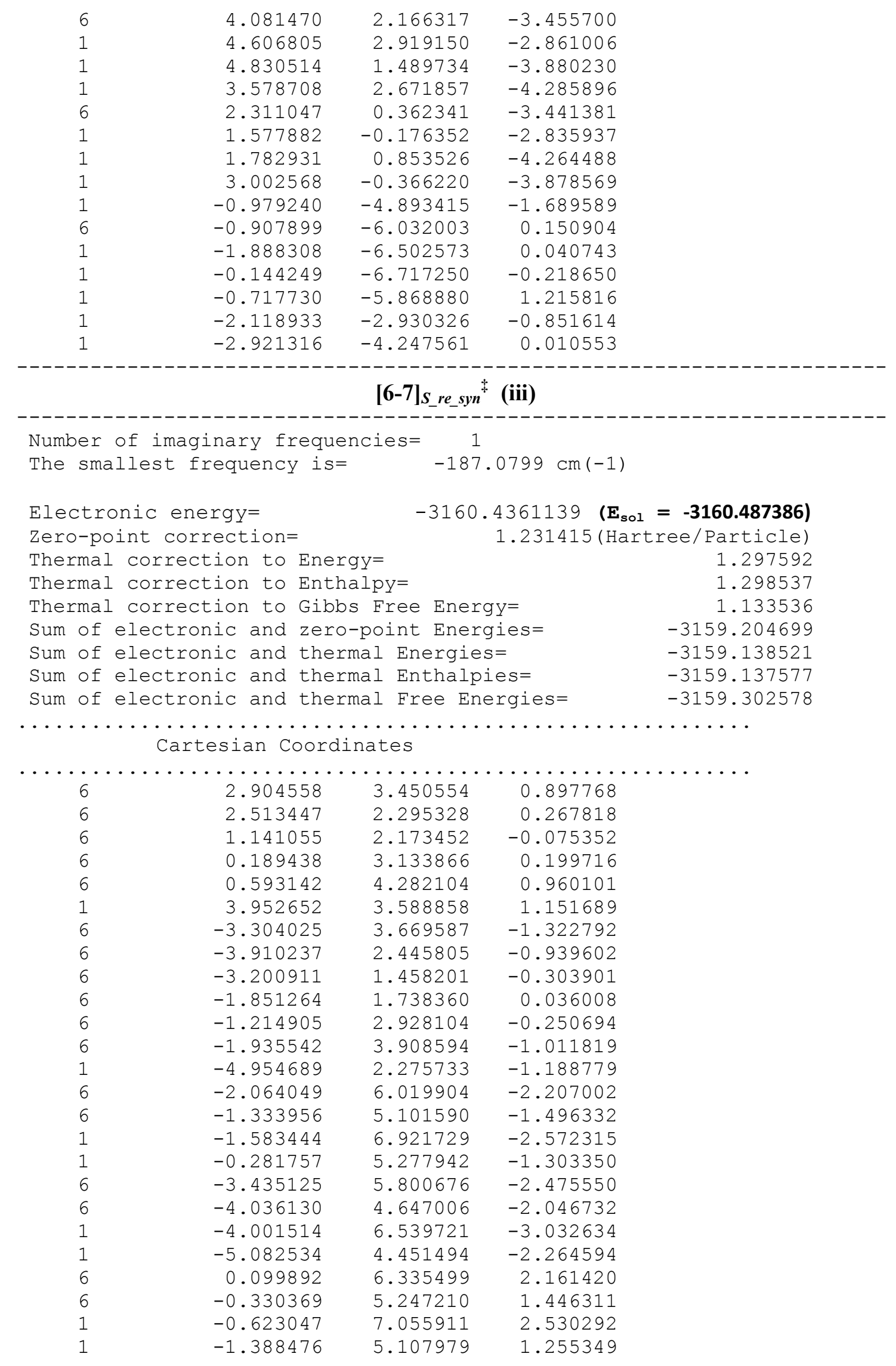




\begin{tabular}{|c|c|c|c|}
\hline 6 & 1.475171 & 6.523492 & 2.432493 \\
\hline 6 & 2.386121 & 5.595269 & 2.003351 \\
\hline 1 & 1.800799 & 7.392924 & 2.994174 \\
\hline 1 & 3.443559 & 5.711414 & 2.224584 \\
\hline 8 & -1.177410 & 0.767114 & 0.750366 \\
\hline 8 & 0.776584 & 1.043479 & -0.782457 \\
\hline 15 & -0.058066 & -0.116569 & -0.025385 \\
\hline 8 & -0.657085 & -0.992240 & -1.093016 \\
\hline 8 & 0.737839 & -0.795853 & 1.064328 \\
\hline 1 & 0.424297 & -2.157865 & 1.156694 \\
\hline 8 & 0.033987 & -3.150180 & 1.164653 \\
\hline 6 & -1.402124 & -3.077012 & 1.234859 \\
\hline 6 & -1.928923 & -3.689072 & -0.065719 \\
\hline 6 & -0.865620 & -4.699863 & -0.491897 \\
\hline 6 & 0.476100 & -3.991747 & -0.321460 \\
\hline 8 & 0.809764 & -3.027799 & -1.153073 \\
\hline 1 & -1.720639 & -3.638066 & 2.118108 \\
\hline 1 & -1.714276 & -2.033373 & 1.343988 \\
\hline 6 & 1.970970 & 4.448826 & 1.275271 \\
\hline 8 & 1.447725 & -4.823983 & 0.009554 \\
\hline 6 & 2.856374 & -4.426742 & 0.103729 \\
\hline 6 & 3.439070 & -4.159511 & -1.282046 \\
\hline 1 & 3.110623 & -4.930985 & -1.984350 \\
\hline 1 & 3.147348 & -3.179763 & -1.661806 \\
\hline 1 & 4.530789 & -4.201710 & -1.213913 \\
\hline 6 & 3.490100 & -5.672213 & 0.714118 \\
\hline 1 & 4.560356 & -5.507507 & 0.862179 \\
\hline 1 & 3.030252 & -5.896516 & 1.679722 \\
\hline 1 & 3.355594 & -6.532380 & 0.052539 \\
\hline 6 & 3.013716 & -3.230432 & 1.029618 \\
\hline 1 & 2.598559 & -2.316164 & 0.596861 \\
\hline 1 & 2.535982 & -3.424698 & 1.994357 \\
\hline 1 & 4.080699 & -3.056052 & 1.199679 \\
\hline 1 & 0.126617 & -2.261102 & -1.221769 \\
\hline 6 & 3.476356 & 1.188495 & -0.015426 \\
\hline 6 & 3.797029 & 0.837777 & -1.338693 \\
\hline 6 & 4.083446 & 0.504596 & 1.060871 \\
\hline 6 & 4.747930 & -0.161277 & -1.567093 \\
\hline 6 & 5.038691 & -0.468965 & 0.778545 \\
\hline 6 & 5.392686 & -0.818680 & -0.524699 \\
\hline 1 & 4.990817 & -0.415414 & -2.594601 \\
\hline 1 & 5.516766 & -0.990316 & 1.607134 \\
\hline 6 & -3.798768 & 0.124305 & 0.004357 \\
\hline 6 & -3.999462 & -0.272925 & 1.343478 \\
\hline 6 & -4.173303 & -0.733558 & -1.047025 \\
\hline 6 & -4.597992 & -1.505106 & 1.595683 \\
\hline 6 & -4.799451 & -1.946517 & -0.739932 \\
\hline 6 & -5.023108 & -2.352485 & 0.570819 \\
\hline 1 & -4.754258 & -1.814539 & 2.628477 \\
\hline 1 & -5.097889 & -2.594446 & -1.559334 \\
\hline 6 & -5.720184 & -3.655203 & 0.930002 \\
\hline 1 & -5.106541 & -4.143643 & 1.701306 \\
\hline 6 & -3.634831 & 0.611405 & 2.527384 \\
\hline 1 & -3.158986 & 1.520197 & 2.149385 \\
\hline 6 & 3.178392 & 1.523875 & -2.547022 \\
\hline 1 & 2.448858 & 2.258658 & -2.196155 \\
\hline 6 & 3.735325 & 0.771930 & 2.519715 \\
\hline 1 & 2.895103 & 1.470074 & 2.550842 \\
\hline
\end{tabular}




\begin{tabular}{|c|c|c|c|}
\hline 6 & 6.468300 & -1.870211 & -0.745306 \\
\hline 1 & 6.190502 & -2.746120 & -0.138776 \\
\hline 6 & -3.929767 & -0.402730 & -2.513976 \\
\hline 1 & -3.316287 & 0.500486 & -2.564122 \\
\hline 6 & -5.253685 & -0.123491 & -3.236381 \\
\hline 1 & -5.814076 & 0.682994 & -2.754773 \\
\hline 1 & -5.070643 & 0.159220 & -4.277512 \\
\hline 1 & -5.890732 & -1.014696 & -3.237015 \\
\hline 6 & -3.149753 & -1.511417 & -3.230696 \\
\hline 1 & -3.698807 & -2.459406 & -3.228054 \\
\hline L & -2.983094 & -1.231799 & -4.27575 \\
\hline 1 & -2.177301 & -1.658693 & -2.756287 \\
\hline 6 & -7.097249 & -3.364893 & 1.543755 \\
\hline 1 & -7.586840 & -4.291995 & 1.857342 \\
\hline 1 & -7.018495 & -2.705785 & 2.412007 \\
\hline 1 & -7.737228 & -2.871004 & 0.805363 \\
\hline 6 & -5.869009 & -4.627419 & -0.239583 \\
\hline 1 & -6.555150 & -4.227859 & -0.993764 \\
\hline 1 & -4.913037 & -4.833003 & -0.731065 \\
\hline 1 & -6.282824 & -5.576354 & 0.111991 \\
\hline 6 & 6.602276 & -2.325358 & -2.197403 \\
\hline 1 & 7.320004 & -3.147385 & -2.269571 \\
\hline 1 & 5.648506 & -2.665912 & -2.610317 \\
\hline 1 & 6.971924 & -1.509154 & -2.827461 \\
\hline 6 & 7.822879 & -1.359464 & -0.233268 \\
\hline 1 & 8.128360 & -0.478240 & -0.806631 \\
\hline 1 & 7.774568 & -1.072087 & 0.820123 \\
\hline 1 & 8.595048 & -2.126797 & -0.345483 \\
\hline 6 & -2.638042 & -0.072407 & 3.469791 \\
\hline 1 & -1.703697 & -0.305661 & 2.952468 \\
\hline 1 & -2.402315 & 0.586547 & 4.311064 \\
\hline 1 & -3.053295 & -0.998730 & 3.882222 \\
\hline 6 & -4.899288 & 1.039256 & 3.283841 \\
\hline 1 & -5.604933 & 1.541909 & 2.616291 \\
\hline 1 & -5.408342 & 0.173690 & 3.721139 \\
\hline 1 & -4.644779 & 1.724810 & 4.097850 \\
\hline 6 & 4.916702 & 1.414806 & 3.256087 \\
\hline 1 & 5.781018 & 0.741952 & 3.264092 \\
\hline 1 & 5.230059 & 2.348076 & 2.778854 \\
\hline 1 & 4.650624 & 1.632752 & 4.294946 \\
\hline 6 & 3.280715 & -0.505016 & 3.236013 \\
\hline 1 & 2.411155 & -0.933133 & 2.732600 \\
\hline 1 & 4.078048 & -1.255916 & 3.264714 \\
\hline 1 & 3.008021 & -0.274690 & 4.270960 \\
\hline 6 & 4.241338 & 2.287703 & -3.346433 \\
\hline 1 & 4.757491 & 3.018295 & -2.717067 \\
\hline 1 & 4.993130 & 1.605023 & -3.756233 \\
\hline 1 & 3.779393 & 2.818492 & -4.184616 \\
\hline 6 & 2.436340 & 0.521417 & -3.438838 \\
\hline 1 & 1.672066 & -0.016513 & -2.87279 \\
\hline 1 & 1.947300 & 1.042179 & -4.26792 \\
\hline 1 & 3.129192 & -0.209770 & -3.869438 \\
\hline 1 & -0.949576 & -4.918751 & -1.561769 \\
\hline 6 & -0.937641 & -5.991908 & 0.318959 \\
\hline 1 & -1.928661 & -6.439755 & 0.20876 \\
\hline 1 & -0.187142 & -6.710265 & -0.01277 \\
\hline 1 & -0.760651 & -5.794902 & 1.38036 \\
\hline 1 & -2.060952 & -2.904397 & -0.81391 \\
\hline
\end{tabular}




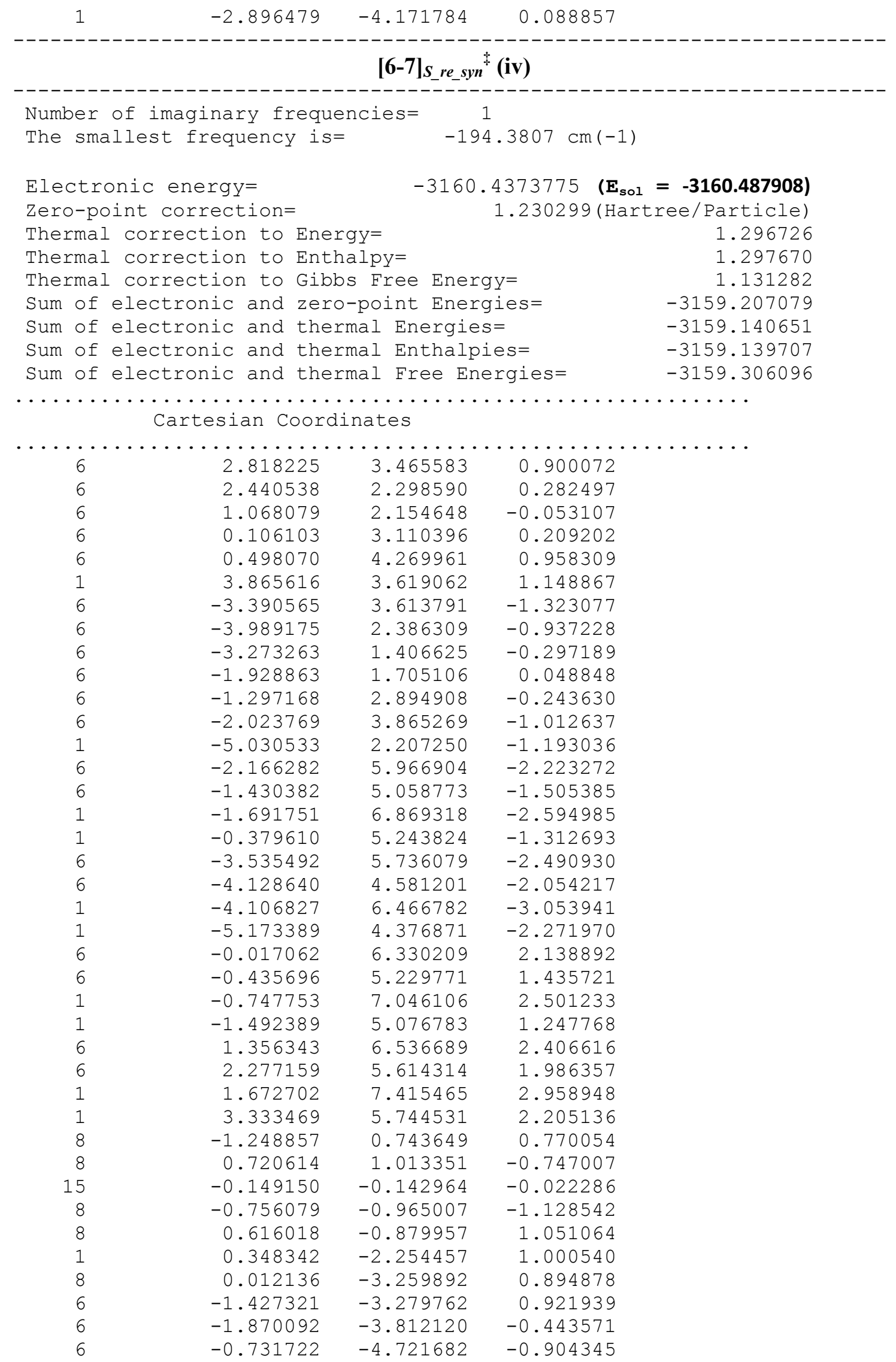




\begin{tabular}{|c|c|c|c|}
\hline 6 & 0.551586 & -3.937718 & -0.641937 \\
\hline 8 & 0.824750 & -2.885072 & -1.384463 \\
\hline 1 & -1.730901 & -3.931170 & 1.746537 \\
\hline 1 & -1.810564 & -2.270560 & 1.105796 \\
\hline 6 & 1.874056 & 4.455792 & 1.270471 \\
\hline 8 & 1.577701 & -4.717797 & -0.353680 \\
\hline 6 & 2.947046 & -4.218594 & -0.16946 \\
\hline 6 & 3.558677 & -3.808661 & -1.506088 \\
\hline 1 & 3.384185 & -4.585157 & -2.256456 \\
\hline 1 & 3.153540 & -2.861811 & -1.863262 \\
\hline 1 & 4.639929 & -3.696264 & -1.37194 \\
\hline 6 & 3.651757 & -5.452621 & 0.38320 \\
\hline 1 & 4.706001 & -5.228814 & 0.56586 \\
\hline 1 & 3.189727 & -5.764312 & 1.323222 \\
\hline 1 & 3.588053 & -6.278898 & -0.330003 \\
\hline 6 & 2.973503 & -3.081502 & 0.840600 \\
\hline 1 & 2.537393 & -2.162487 & 0.440421 \\
\hline 1 & 2.445366 & -3.366546 & 1.755172 \\
\hline 1 & 4.012857 & -2.859858 & 1.096850 \\
\hline 1 & 0.092185 & -2.161319 & -1.382501 \\
\hline 6 & 3.432949 & 1.214690 & 0.00523 \\
\hline 6 & 3.755776 & 0.852905 & -1.319169 \\
\hline 6 & 4.089982 & 0.586552 & 1.08215 \\
\hline 6 & 4.764033 & -0.085311 & -1.531907 \\
\hline 6 & 5.116451 & -0.324584 & 0.81330 \\
\hline 6 & 5.479002 & -0.666384 & -0.48548 \\
\hline 1 & 5.023981 & -0.358898 & -2.553710 \\
\hline 1 & 5.629017 & -0.788158 & 1.652848 \\
\hline 6 & -3.842980 & 0.057024 & 0.001762 \\
\hline 6 & -4.040544 & -0.351975 & 1.33668 \\
\hline 6 & -4.179142 & -0.810048 & -1.05786 \\
\hline 6 & -4.600867 & -1.604882 & 1.586238 \\
\hline 6 & -4.743882 & -2.051224 & -0.753408 \\
\hline 6 & -4.964190 & -2.470427 & 0.55533 \\
\hline 1 & -4.769699 & -1.905274 & 2.618431 \\
\hline 1 & -5.017983 & -2.719263 & -1.568222 \\
\hline 6 & -5.638604 & -3.799994 & 0.83955 \\
\hline 1 & -5.652201 & -4.365360 & -0.101887 \\
\hline 6 & -3.703603 & 0.535068 & 2.525795 \\
\hline 1 & -3.238694 & 1.452622 & 2.15558 \\
\hline 6 & 3.084065 & 1.473054 & -2.534529 \\
\hline 1 & 2.326937 & 2.182961 & -2.192087 \\
\hline 6 & 3.712857 & 0.829320 & 2.537930 \\
\hline 1 & 2.857349 & 1.508501 & 2.56389 \\
\hline 6 & 6.654792 & -1.572721 & -0.80728 \\
\hline 1 & 6.396203 & -2.120134 & -1.724029 \\
\hline 6 & -3.980109 & -0.445040 & -2.523541 \\
\hline 1 & -3.402284 & 0.481350 & -2.570466 \\
\hline 6 & -5.334895 & -0.198550 & -3.20087 \\
\hline 1 & -5.912983 & 0.570031 & -2.67970 \\
\hline 1 & -5.193524 & 0.121480 & -4.237765 \\
\hline 1 & -5.936041 & -1.114369 & -3.209690 \\
\hline 6 & -3.182836 & -1.504191 & -3.29262 \\
\hline 1 & -3.682092 & -2.479255 & -3.27859 \\
\hline 1 & -3.082987 & -1.202687 & -4.340095 \\
\hline 1 & -2.182199 & -1.607012 & -2.868661 \\
\hline 6 & -4.894513 & -4.639448 & 1.882138 \\
\hline 1 & -5.403127 & -5.595998 & 2.03467 \\
\hline
\end{tabular}




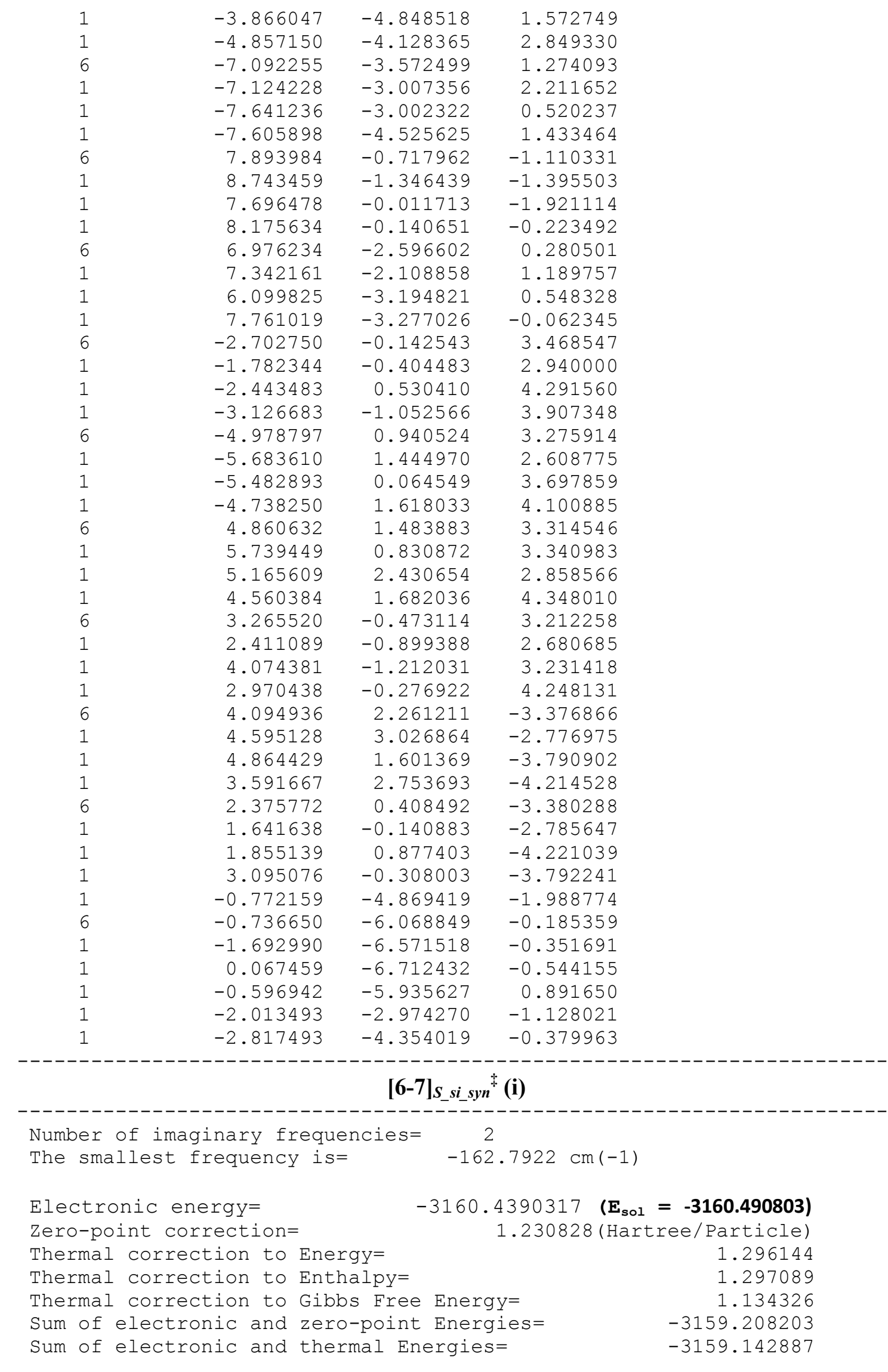


$\begin{array}{ll}\text { Sum of electronic and thermal Enthalpies= } & -3159.141943 \\ \text { Sum } & -3159.304706\end{array}$

Sum of electronic and thermal Free Energies $=$

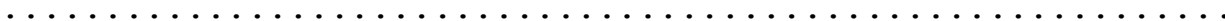

Cartesian Coordinates

\begin{tabular}{|c|c|c|c|}
\hline 6 & 1.280453 & 4.688129 & 1.446429 \\
\hline 6 & 2.352611 & 3.828738 & 1.086883 \\
\hline 6 & 2.136008 & 2.643940 & 0.431547 \\
\hline 6 & 0.801970 & 2.357996 & 0.034604 \\
\hline 6 & -0.279114 & 3.173818 & 0.291544 \\
\hline 6 & -0.053402 & 4.350885 & 1.080724 \\
\hline 1 & 3.365725 & 4.115254 & 1.354355 \\
\hline 6 & -3.724368 & 3.228916 & -1.428735 \\
\hline 6 & -4.162837 & 1.919566 & -1.105475 \\
\hline 6 & -3.356443 & 1.034319 & -0.434629 \\
\hline 6 & -2.092561 & 1.507420 & 0.004983 \\
\hline 6 & -1.621545 & 2.783862 & -0.225581 \\
\hline 6 & -2.430668 & 3.662209 & -1.021369 \\
\hline 1 & -5.148187 & 1.598886 & -1.434138 \\
\hline 6 & -2.788574 & 5.749550 & -2.212546 \\
\hline 6 & -1.980752 & 4.937891 & -1.457471 \\
\hline 1 & -2.421539 & 6.716432 & -2.541188 \\
\hline 1 & -0.979923 & 5.262628 & -1.196419 \\
\hline 6 & -4.090360 & 5.334859 & -2.577677 \\
\hline 6 & -4.541801 & 4.098873 & -2.197545 \\
\hline 1 & -4.719734 & 5.989209 & -3.171854 \\
\hline 1 & -5.529247 & 3.754107 & -2.491746 \\
\hline 6 & -0.848053 & 6.310102 & 2.276858 \\
\hline 6 & -1.109202 & 5.184298 & 1.537453 \\
\hline 1 & -1.670075 & 6.929999 & 2.619944 \\
\hline 1 & -2.133126 & 4.916353 & 1.301027 \\
\hline 6 & 0.481047 & 6.666777 & 2.602121 \\
\hline 6 & 1.518447 & 5.867849 & 2.198313 \\
\hline 1 & 0.674421 & 7.563675 & 3.181397 \\
\hline 1 & 2.543806 & 6.115457 & 2.459238 \\
\hline 8 & -1.334881 & 0.627747 & 0.751038 \\
\hline 8 & 0.612188 & 1.204861 & -0.705710 \\
\hline 15 & -0.029999 & -0.055382 & 0.078177 \\
\hline 8 & -0.380358 & -1.054537 & -0.998450 \\
\hline 8 & 0.789824 & -0.532285 & 1.247311 \\
\hline 1 & 0.020212 & -2.354475 & -0.853230 \\
\hline 6 & -3.763972 & -0.386316 & -0.209121 \\
\hline 6 & -3.948603 & -1.237482 & -1.321130 \\
\hline 6 & -4.004493 & -0.869321 & 1.089771 \\
\hline 6 & -4.451892 & -2.519931 & -1.105450 \\
\hline 6 & -4.472612 & -2.177357 & 1.254043 \\
\hline 6 & -4.731094 & -3.010838 & 0.170138 \\
\hline 6 & 3.227278 & 1.671716 & 0.109042 \\
\hline 6 & 3.784103 & 0.868697 & 1.125382 \\
\hline 6 & 3.661027 & 1.528411 & -1.221321 \\
\hline 6 & 4.799448 & -0.026427 & 0.785752 \\
\hline 6 & 4.681895 & 0.621915 & -1.508991 \\
\hline 6 & 5.266643 & -0.163922 & -0.519728 \\
\hline 8 & 0.129466 & -3.407400 & -0.656105 \\
\hline 1 & 5.019014 & 0.525846 & -2.538360 \\
\hline 1 & 5.232117 & -0.655744 & 1.560142 \\
\hline 1 & -4.674531 & -2.529866 & 2.261880 \\
\hline 1 & -4.636200 & -3.165228 & -1.963602 \\
\hline
\end{tabular}




\begin{tabular}{|c|c|c|c|}
\hline 6 & 3.056551 & 2.326491 & -2.366516 \\
\hline 1 & 2.236130 & 2.933832 & -1.973173 \\
\hline 6 & 3.299762 & 0.932267 & 2.568008 \\
\hline 1 & 2.261787 & 1.279625 & 2.552432 \\
\hline 6 & 6.408668 & -1.115900 & -0.822681 \\
\hline 1 & 6.436954 & -1.853351 & -0.009332 \\
\hline 6 & 2.470380 & 1.402167 & -3.439785 \\
\hline 1 & 1.736599 & 0.718702 & -3.005112 \\
\hline | & 1.977876 & 1.991730 & -4.219277 \\
\hline 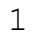 & 3.255572 & 0.808357 & -3.919817 \\
\hline & 4.089149 & 3.287692 & -2.967536 \\
\hline & 4.478446 & 3.971664 & -2.207934 \\
\hline 1 & 4.936357 & 2.737533 & -3.390379 \\
\hline 1 & 3.640006 & 3.881026 & -3.769970 \\
\hline 6 & 3.311246 & -0.441034 & 3.249979 \\
\hline 1 & 4.326537 & -0.736866 & 3.536947 \\
\hline 1 & 2.712257 & -0.407061 & 4.164998 \\
\hline 1 & 2.903633 & -1.214160 & 2.596157 \\
\hline 6 & 4.141871 & 1.911898 & 3.396568 \\
\hline 1 & 3.805722 & 1.918201 & 4.438372 \\
\hline 1 & 5.194691 & 1.609272 & 3.380827 \\
\hline 1 & 4.079487 & 2.932789 & 3.014745 \\
\hline 6 & 7.743129 & -0.359008 & -0.819275 \\
\hline 1 & 7.744617 & 0.404520 & -1.604544 \\
\hline 1 & 7.906768 & 0.143528 & 0.137776 \\
\hline 1 & 8.580279 & -1.040082 & -1.002678 \\
\hline 6 & 6.223670 & -1.874801 & -2.138246 \\
\hline 1 & 6.991334 & -2.647784 & -2.243341 \\
\hline 1 & 5.240486 & -2.351504 & -2.185319 \\
\hline 1 & 6.316260 & -1.205295 & -2.999441 \\
\hline 6 & -3.611502 & -0.825847 & -2.748417 \\
\hline 1 & -3.111570 & 0.145794 & -2.716292 \\
\hline 6 & -3.842781 & -0.005519 & 2.332789 \\
\hline 1 & -3.489509 & 0.982273 & 2.025950 \\
\hline 6 & -5.286647 & -4.418640 & 0.317259 \\
\hline 1 & -6.067637 & -4.528442 & -0.447073 \\
\hline 6 & -4.202943 & -5.463737 & 0.021673 \\
\hline 1 & -4.618590 & -6.475661 & 0.050579 \\
\hline 1 & -3.750609 & -5.305760 & -0.961859 \\
\hline 1 & -3.409263 & -5.403297 & 0.775031 \\
\hline 6 & -5.921991 & -4.691822 & 1.679174 \\
\hline 1 & -5.165608 & -4.694945 & 2.471773 \\
\hline 1 & -6.674982 & -3.939849 & 1.930787 \\
\hline 1 & -6.401955 & -5.674218 & 1.681337 \\
\hline 6 & -2.805072 & -0.586711 & 3.300198 \\
\hline 1 & -3.081810 & -1.599088 & 3.616040 \\
\hline 1 & -1.815090 & -0.620619 & 2.837785 \\
\hline 1 & -2.738482 & 0.034368 & 4.198890 \\
\hline 6 & -5.195055 & 0.193339 & 3.029958 \\
\hline 1 & -5.933086 & 0.613132 & 2.340691 \\
\hline 1 & -5.589092 & -0.755371 & 3.408776 \\
\hline 1 & -5.089076 & 0.874466 & 3.879617 \\
\hline 6 & -4.878475 & -0.684468 & -3.600058 \\
\hline 1 & -4.624350 & -0.359657 & -4.613524 \\
\hline$\perp$ & -5.404088 & -1.642545 & -3.676482 \\
\hline 1 & -5.575003 & 0.042124 & -3.171935 \\
\hline 6 & -2.632213 & -1.812319 & -3.396702 \\
\hline 1 & -2.372216 & -1.472696 & -4.40407 \\
\hline
\end{tabular}




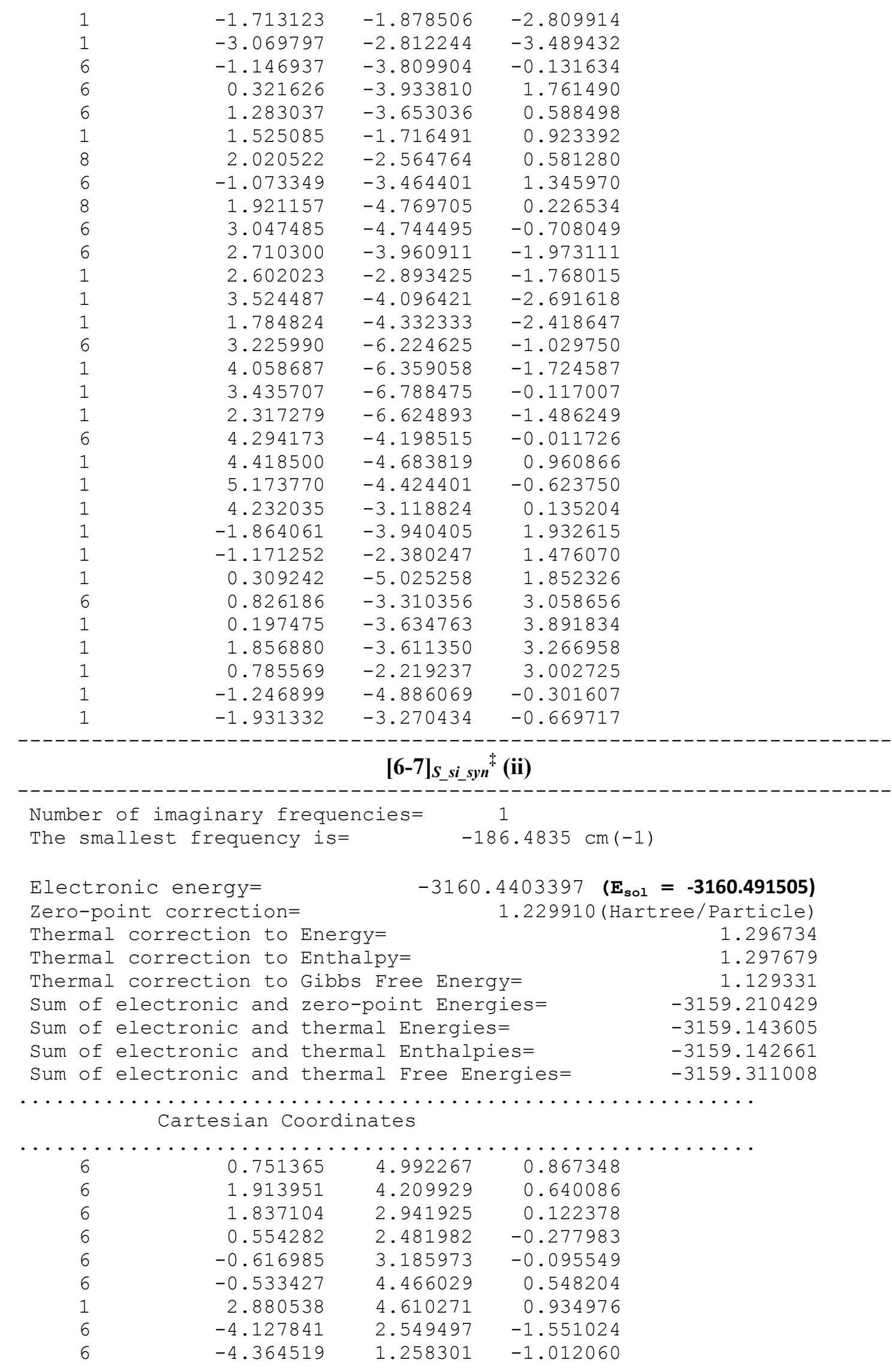




\begin{tabular}{|c|c|c|c|}
\hline 6 & -3.399867 & 0.584697 & -0.306050 \\
\hline 6 & -2.183070 & 1.270000 & -0.049620 \\
\hline 6 & -1.908933 & 2.551515 & -0.483652 \\
\hline 6 & -2.880999 & 3.195842 & -1.318538 \\
\hline 1 & -5.328844 & 0.787594 & -1.182500 \\
\hline 6 & -3.600975 & 5.034910 & -2.732528 \\
\hline 6 & -2.639779 & 4.444595 & -1.952065 \\
\hline 1 & -3.394698 & 5.986059 & -3.212578 \\
\hline 1 & -1.676697 & 4.924828 & -1.818124 \\
\hline 6 & -4.856411 & 4.412285 & -2.924685 \\
\hline 6 & -5.107996 & 3.194727 & -2.349599 \\
\hline 1 & -5.610147 & 4.894050 & -3.538810 \\
\hline 1 & -6.058891 & 2.693095 & -2.506814 \\
\hline 6 & -1.552657 & 6.449282 & 1.514825 \\
\hline 6 & -1.679556 & 5.221579 & 0.916160 \\
\hline 1 & -2.441952 & 7.006803 & 1.790437 \\
\hline 1 & -2.665413 & 4.812517 & 0.726377 \\
\hline 6 & -0.275095 & 6.991831 & 1.784627 \\
\hline 6 & 0.848368 & 6.272368 & 1.473947 \\
\hline 1 & -0.188654 & 7.967229 & 2.252137 \\
\hline 1 & 1.837056 & 6.662562 & 1.699804 \\
\hline 8 & -1.253446 & 0.601091 & 0.726757 \\
\hline 8 & 0.506479 & 1.256984 & -0.913380 \\
\hline 15 & 0.013702 & -0.028901 & -0.064741 \\
\hline 8 & -0.389707 & -1.050795 & -1.097078 \\
\hline 8 & 0.985980 & -0.453716 & 1.007829 \\
\hline 1 & 0.012476 & -2.356369 & -0.770511 \\
\hline 6 & -3.614870 & -0.808112 & 0.197624 \\
\hline 6 & -3.680956 & -1.885629 & -0.712308 \\
\hline 6 & -3.788067 & -1.038790 & 1.574646 \\
\hline 6 & -4.008292 & -3.153403 & -0.228660 \\
\hline 6 & -4.103993 & -2.328746 & 2.009022 \\
\hline 6 & -4.247796 & -3.392768 & 1.124733 \\
\hline 6 & 2.997611 & 2.000888 & 0.069618 \\
\hline 6 & 3.524834 & 1.496059 & 1.278995 \\
\hline 6 & 3.506970 & 1.551992 & -1.161690 \\
\hline 6 & 4.577550 & 0.584231 & 1.221962 \\
\hline 6 & 4.557395 & 0.632380 & -1.164027 \\
\hline 6 & 5.105119 & 0.133862 & 0.013491 \\
\hline 8 & 0.244470 & -3.315171 & -0.399524 \\
\hline 1 & 4.959842 & 0.288874 & -2.115684 \\
\hline 1 & 4.989862 & 0.202497 & 2.152970 \\
\hline 1 & -4.263823 & -2.504702 & 3.071269 \\
\hline 1 & -4.078001 & -3.979218 & -0.932996 \\
\hline 6 & 2.971967 & 2.044719 & -2.497120 \\
\hline 1 & 2.107757 & 2.688061 & -2.306849 \\
\hline 6 & 2.986521 & 1.894552 & 2.647036 \\
\hline 1 & 2.025811 & 2.396845 & 2.504079 \\
\hline 6 & 6.245875 & -0.864305 & -0.016924 \\
\hline 1 & 6.348605 & -1.208377 & -1.055021 \\
\hline 6 & 2.498023 & 0.885002 & -3.380305 \\
\hline 1 & 1.717592 & 0.308452 & -2.877356 \\
\hline 1 & 2.089822 & 1.268424 & -4.320674 \\
\hline 1 & 3.325063 & 0.210284 & -3.628903 \\
\hline 6 & 4.026228 & 2.893801 & -3.218315 \\
\hline 1 & 4.338471 & 3.740109 & -2.599824 \\
\hline 1 & 4.916700 & 2.299708 & -3.449269 \\
\hline 1 & 3.627630 & 3.282953 & -4.160297 \\
\hline
\end{tabular}




\begin{tabular}{|c|c|c|c|}
\hline 6 & 2.723931 & 0.684702 & 3.551970 \\
\hline 1 & 3.652370 & 0.172806 & 3.826674 \\
\hline 1 & 2.250245 & 1.016215 & 4.481437 \\
\hline 1 & 2.059587 & -0.024823 & 3.054075 \\
\hline 6 & 3.944557 & 2.874744 & 3.336189 \\
\hline 1 & 3.549190 & 3.183755 & 4.308838 \\
\hline 1 & 4.919020 & 2.401645 & 3.500363 \\
\hline 1 & 4.111199 & 3.771362 & 2.732359 \\
\hline 6 & 5.948763 & -2.087037 & 0.856373 \\
\hline 1 & 5.947805 & -1.821350 & 1.919243 \\
\hline 1 & 4.966247 & -2.500586 & 0.613764 \\
\hline 1 & 6.709413 & -2.860932 & 0.710540 \\
\hline 6 & 7.564550 & -0.201782 & 0.397338 \\
\hline 1 & 8.392567 & -0.915998 & 0.348305 \\
\hline 1 & 7.799656 & 0.647431 & -0.249761 \\
\hline 1 & 7.496295 & 0.168656 & 1.425740 \\
\hline 6 & -3.406755 & -1.712668 & -2.200193 \\
\hline 1 & -2.873333 & -0.767049 & -2.334706 \\
\hline 6 & -3.669686 & 0.064604 & 2.614218 \\
\hline 1 & -3.420049 & 0.999043 & 2.104898 \\
\hline 6 & -4.638548 & -4.770676 & 1.625800 \\
\hline 1 & -4.905188 & -4.665561 & 2.685159 \\
\hline 6 & -5.863007 & -5.319313 & 0.886330 \\
\hline 1 & -6.178345 & -6.272865 & 1.320518 \\
\hline 1 & -6.701290 & -4.620052 & 0.939653 \\
\hline 1 & -5.637792 & -5.495122 & -0.170434 \\
\hline 6 & -3.465559 & -5.752362 & 1.533302 \\
\hline 1 & -3.131995 & -5.856079 & 0.494621 \\
\hline 1 & -2.616624 & -5.405327 & 2.130392 \\
\hline 1 & -3.756756 & -6.743213 & 1.895585 \\
\hline 6 & -2.541653 & -0.235925 & 3.608108 \\
\hline 1 & -2.759093 & -1.140298 & 4.186802 \\
\hline 1 & -1.591331 & -0.375248 & 3.085962 \\
\hline 1 & -2.427639 & 0.591389 & 4.315308 \\
\hline 6 & -5.002607 & 0.285828 & 3.339173 \\
\hline 1 & -5.800890 & 0.520315 & 2.629264 \\
\hline 1 & -5.301155 & -0.606415 & 3.899003 \\
\hline 1 & -4.918122 & 1.113538 & 4.049868 \\
\hline 6 & -4.714917 & -1.661852 & -3.000792 \\
\hline 1 & -4.506386 & -1.556105 & -4.070031 \\
\hline 1 & -5.283732 & -2.586901 & -2.855091 \\
\hline 1 & -5.349676 & -0.826846 & -2.694998 \\
\hline 6 & -2.508569 & -2.824124 & -2.756420 \\
\hline 1 & -2.179109 & -2.561140 & -3.765994 \\
\hline 1 & -1.623043 & -2.968137 & -2.134569 \\
\hline 1 & -3.043809 & -3.777312 & -2.826312 \\
\hline 6 & -0.542704 & -3.525560 & 0.778558 \\
\hline 6 & 0.419910 & -3.434566 & 1.961203 \\
\hline 1 & 0.060593 & -4.006021 & 2.821323 \\
\hline 1 & 0.547821 & -2.392270 & 2.268861 \\
\hline 6 & 1.745971 & -3.993991 & 1.448928 \\
\hline 6 & 2.011289 & -3.390579 & 0.073982 \\
\hline 1 & 1.887628 & -1.447063 & 0.514424 \\
\hline 8 & 2.425310 & -2.159140 & -0.040562 \\
\hline 1 & -1.343624 & -2.776486 & 0.818870 \\
\hline 1 & -0.994588 & -4.518306 & 0.699461 \\
\hline 8 & 2.525805 & -4.262365 & -0.773610 \\
\hline 6 & 2.928837 & -3.887806 & -2.137578 \\
\hline
\end{tabular}




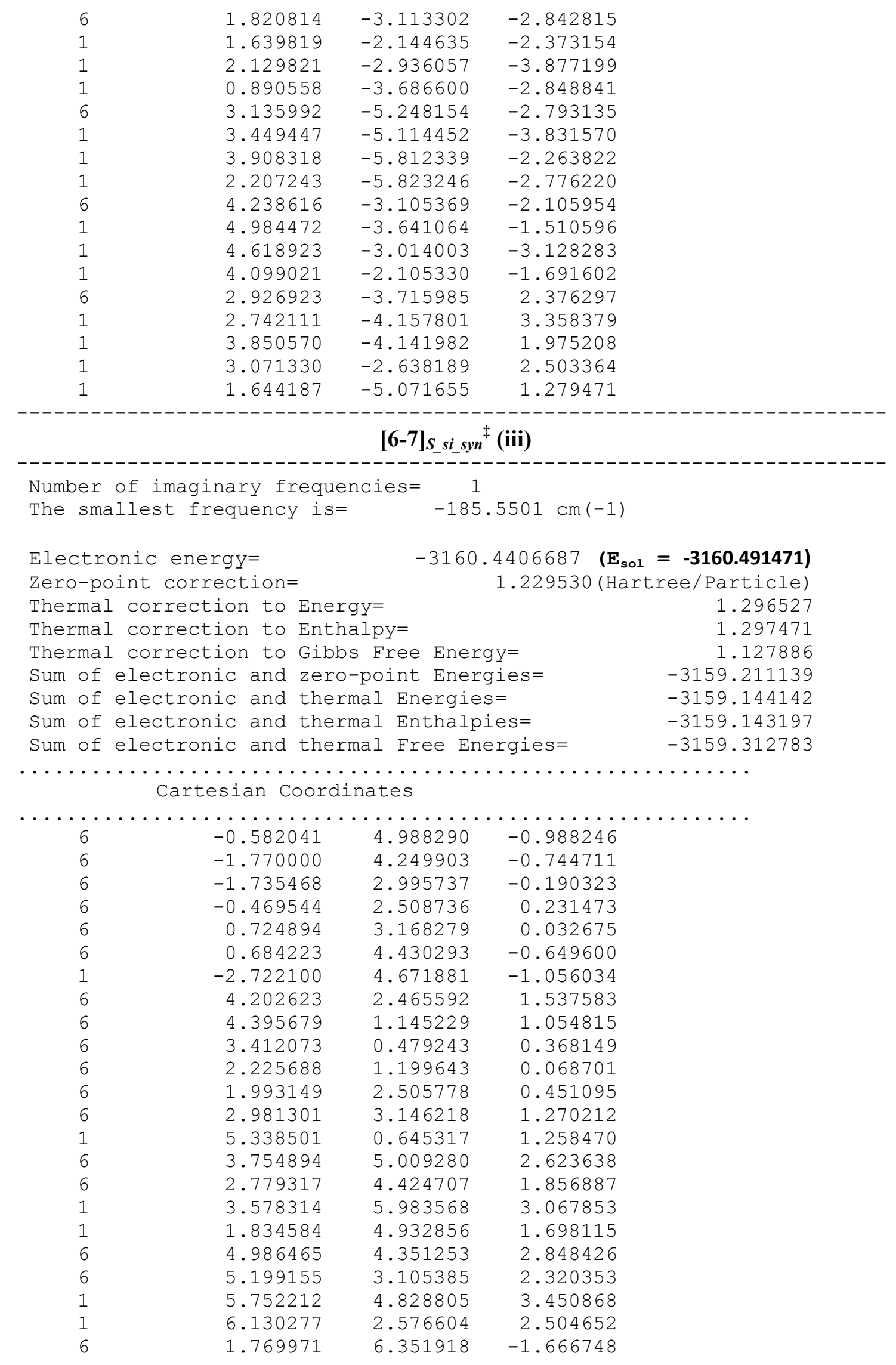




\begin{tabular}{|c|c|c|c|}
\hline 6 & 1.855195 & 5.137505 & -1.034450 \\
\hline 1 & 2.677604 & 6.872806 & -1.954054 \\
\hline 1 & 2.826665 & 4.702141 & -0.829591 \\
\hline 6 & 0.511434 & 6.927615 & -1.956032 \\
\hline 6 & -0.635743 & 6.253423 & -1.630256 \\
\hline 1 & 0.458043 & 7.891963 & -2.450655 \\
\hline 1 & -1.610643 & 6.668732 & -1.870832 \\
\hline 8 & 1.282261 & 0.534560 & -0.693282 \\
\hline 8 & -0.464887 & 1.304529 & 0.907019 \\
\hline 15 & -0.017705 & -0.023343 & 0.100607 \\
\hline 8 & 0.329697 & -1.032054 & 1.166125 \\
\hline 8 & -0.991337 & -0.439287 & -0.974169 \\
\hline 1 & -0.079217 & -2.338703 & 0.837785 \\
\hline 6 & 3.559609 & -0.949443 & -0.053185 \\
\hline 6 & 3.556007 & -1.970791 & 0.919539 \\
\hline 6 & 3.709522 & -1.275991 & -1.414882 \\
\hline 6 & 3.782782 & -3.286673 & 0.509443 \\
\hline 6 & 3.923831 & -2.607897 & -1.773948 \\
\hline 6 & 3.980095 & -3.627274 & -0.826422 \\
\hline 6 & -2.923590 & 2.090793 & -0.120817 \\
\hline 6 & -3.455419 & 1.568432 & -1.320767 \\
\hline 6 & -3.453528 & 1.687557 & 1.117607 \\
\hline 6 & -4.533006 & 0.687168 & -1.248518 \\
\hline 6 & -4.527856 & 0.795974 & 1.135367 \\
\hline 6 & -5.081051 & 0.282484 & -0.033167 \\
\hline 8 & -0.319506 & -3.292044 & 0.459358 \\
\hline 1 & -4.945076 & 0.486060 & 2.092155 \\
\hline 1 & -4.948629 & 0.292118 & -2.172414 \\
\hline 1 & 4.055698 & -2.848353 & -2.826533 \\
\hline 1 & 3.792179 & -4.078840 & 1.255158 \\
\hline 6 & -2.914388 & 2.199467 & 2.444156 \\
\hline 1 & -2.029723 & 2.811356 & 2.244465 \\
\hline 6 & -2.893930 & 1.912888 & -2.694259 \\
\hline 1 & -1.916617 & 2.383350 & -2.555852 \\
\hline 6 & -6.249891 & -0.682005 & 0.014608 \\
\hline 1 & -6.360365 & -1.006740 & 1.058141 \\
\hline 6 & -2.482643 & 1.049715 & 3.361291 \\
\hline 1 & -1.722330 & 0.431735 & 2.877009 \\
\hline 1 & -2.063021 & 1.445370 & 4.291478 \\
\hline 1 & -3.333261 & 0.411942 & 3.626940 \\
\hline 6 & -3.948374 & 3.097832 & 3.134213 \\
\hline 1 & -4.231974 & 3.936323 & 2.491746 \\
\hline 1 & -4.857050 & 2.535600 & 3.373760 \\
\hline 1 & -3.545369 & 3.499796 & 4.068945 \\
\hline 6 & -2.668809 & 0.672032 & -3.566458 \\
\hline 1 & -3.613455 & 0.194301 & -3.847141 \\
\hline 1 & -2.164636 & 0.960844 & -4.494048 \\
\hline 1 & -2.044644 & -0.053232 & -3.040116 \\
\hline 6 & -3.810469 & 2.909407 & -3.415584 \\
\hline 1 & -3.401386 & 3.171780 & -4.396289 \\
\hline 1 & -4.803021 & 2.471150 & -3.567635 \\
\hline 1 & -3.940822 & 3.830216 & -2.840037 \\
\hline 6 & -5.991534 & -1.926281 & -0.840359 \\
\hline 1 & -5.993331 & -1.678476 & -1.907522 \\
\hline 1 & -5.017814 & -2.360673 & -0.598876 \\
\hline 1 & -6.770234 & -2.678102 & -0.675699 \\
\hline 6 & -7.549578 & 0.012590 & -0.407279 \\
\hline 1 & -8.397925 & -0.676532 & -0.347452 \\
\hline
\end{tabular}




\begin{tabular}{|c|c|c|c|}
\hline 1 & -7.759213 & 0.877173 & 0.228164 \\
\hline 1 & -7.471924 & 0.366495 & -1.440850 \\
\hline 6 & 3.294366 & -1.694163 & 2.393900 \\
\hline 1 & 2.816234 & -0.713064 & 2.469697 \\
\hline D & 3.648349 & -0.232917 & -2.519845 \\
\hline & 3.461041 & 0.744223 & -2.066081 \\
\hline 6 & 4.275165 & -5.062674 & -1.218939 \\
\hline 1 & 3.984083 & -5.695273 & -0.370337 \\
\hline 6 & 3.482859 & -5.524765 & -2.445081 \\
\hline$\perp$ & 3.650334 & -6.590399 & -2.626456 \\
\hline & 2.408702 & -5.363283 & -2.314537 \\
\hline & 3.795914 & -4.987162 & -3.345509 \\
\hline 6 & 5.779903 & -5.247234 & -1.453879 \\
\hline 1 & 6.111245 & -4.618959 & -2.287657 \\
\hline 1 & 6.353211 & -4.959803 & -0.568724 \\
\hline 1 & 6.013271 & -6.288495 & -1.697271 \\
\hline 6 & 2.491267 & -0.524946 & -3.483302 \\
\hline 1 & 2.644826 & -1.476475 & -4.004303 \\
\hline 1 & 1.540983 & -0.571591 & -2.944255 \\
\hline 1 & 2.418480 & 0.261282 & -4.241010 \\
\hline 6 & 4.983038 & -0.140956 & -3.268649 \\
\hline 1 & 5.803530 & 0.087320 & -2.582572 \\
\hline 1 & 5.218605 & -1.083542 & -3.773692 \\
\hline 1 & 4.940385 & 0.644275 & -4.029530 \\
\hline 6 & 4.603515 & -1.672319 & 3.193799 \\
\hline 1 & 4.402315 & -1.485887 & 4.253256 \\
\hline 1 & 5.113825 & -2.638173 & 3.109451 \\
\hline 1 & 5.289082 & -0.900116 & 2.836924 \\
\hline 6 & 2.331712 & -2.715342 & 3.012489 \\
\hline 1 & 2.012366 & -2.369503 & 4.000039 \\
\hline 1 & 1.443701 & -2.844816 & 2.391307 \\
\hline 1 & 2.810526 & -3.691054 & 3.147451 \\
\hline 6 & 0.468815 & -3.497991 & -0.71955 \\
\hline 6 & -0.495948 & -3.431912 & -1.903044 \\
\hline 1 & -0.137088 & -4.021018 & -2.751325 \\
\hline 1 & -0.621403 & -2.396154 & -2.233027 \\
\hline 6 & -1.823774 & -3.975811 & -1.379559 \\
\hline 6 & -2.082513 & -3.344903 & -0.016433 \\
\hline 1 & -1.917947 & -1.409297 & -0.479844 \\
\hline 8 & -2.476447 & -2.104504 & 0.07512 \\
\hline 1 & 1.255027 & -2.734815 & -0.768225 \\
\hline 1 & 0.942624 & -4.481072 & -0.632299 \\
\hline 8 & -2.618775 & -4.192752 & 0.841942 \\
\hline 6 & -3.029019 & -3.786360 & 2.194503 \\
\hline 6 & -1.910903 & -3.024533 & 2.89732 \\
\hline 1 & -1.707933 & -2.065648 & 2.416382 \\
\hline 1 & -2.221351 & -2.828481 & 3.927822 \\
\hline 1 & -0.992401 & -3.616528 & 2.915465 \\
\hline 6 & -3.271976 & -5.131033 & 2.869890 \\
\hline 1 & -3.590365 & -4.974146 & 3.903540 \\
\hline 1 & -4.052464 & -5.686284 & 2.343106 \\
\hline 1 & -2.356140 & -5.726745 & 2.869450 \\
\hline 6 & -4.321383 & -2.976456 & 2.136110 \\
\hline 1 & -5.070611 & -3.503678 & 1.53735 \\
\hline 1 & -4.712851 & -2.865448 & 3.152238 \\
\hline 1 & -4.156005 & -1.984413 & 1.711477 \\
\hline 6 & -3.005618 & -3.706208 & -2.308209 \\
\hline 1 & -2.83 & -4.172206 & -3 \\
\hline
\end{tabular}

S81 


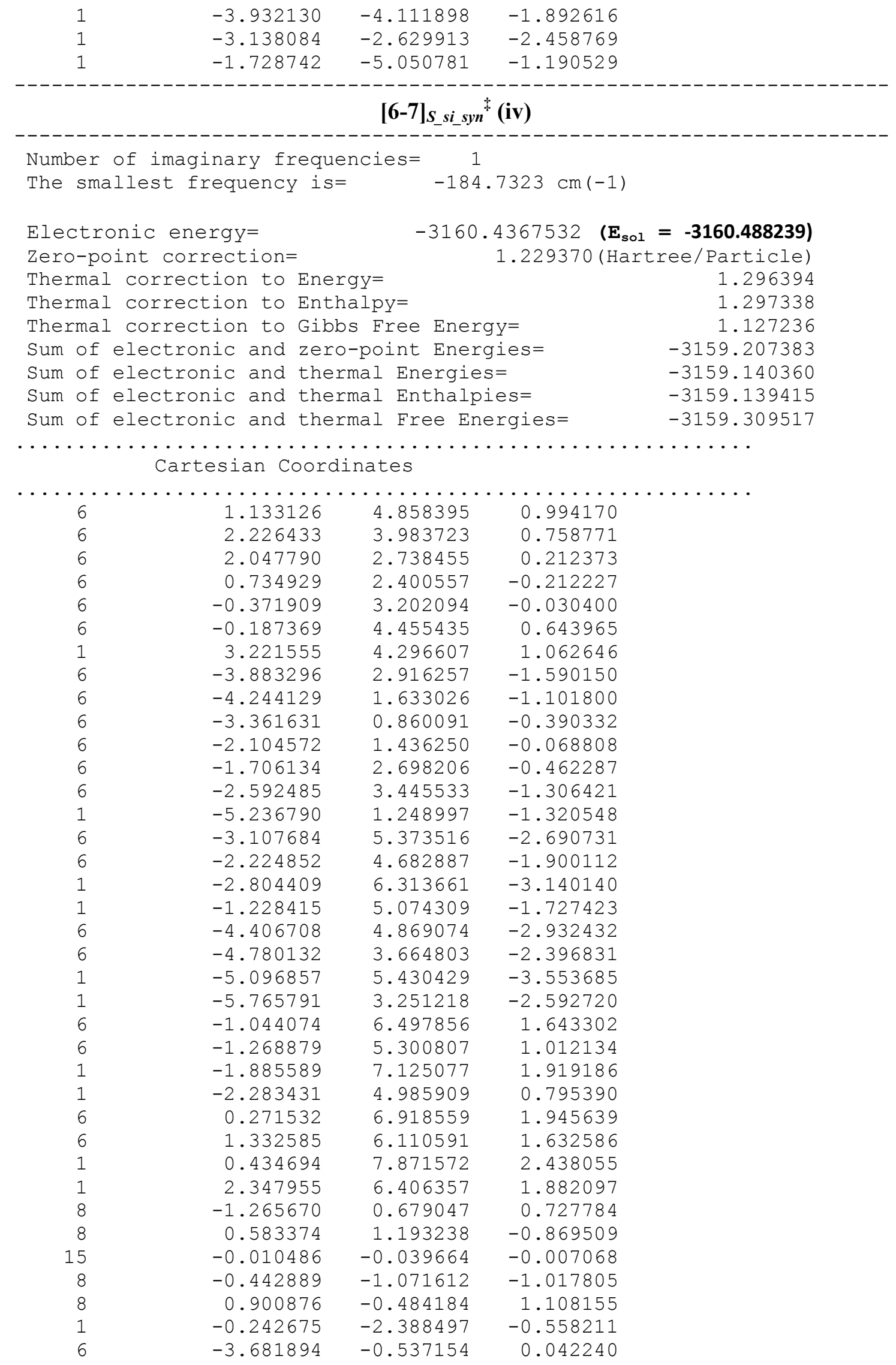




\begin{tabular}{|c|c|c|c|}
\hline 6 & -3.702116 & -1.578407 & -0.906378 \\
\hline 6 & -3.946842 & -0.815083 & 1.397622 \\
\hline 6 & -4.065011 & -2.863403 & -0.490938 \\
\hline 6 & -4.295473 & -2.114888 & 1.763555 \\
\hline 6 & -4.377357 & -3.151418 & 0.834638 \\
\hline 6 & 3.139293 & 1.718932 & 0.124284 \\
\hline 6 & 3.601255 & 1.096468 & 1.306823 \\
\hline 6 & 3.658449 & 1.330689 & -1.119148 \\
\hline 6 & 4.604866 & 0.137798 & 1.205772 \\
\hline 6 & 4.679554 & 0.375297 & -1.166023 \\
\hline 6 & 5.173997 & -0.229726 & -0.016260 \\
\hline 8 & -0.122440 & -3.325712 & -0.087688 \\
\hline 1 & 5.086193 & 0.102264 & -2.135443 \\
\hline 1 & 4.957402 & -0.349341 & 2.112087 \\
\hline 1 & -4.505649 & -2.331892 & 2.81034 \\
\hline 1 & -4.081833 & -3.659927 & -1.228111 \\
\hline 6 & 3.161135 & 1.920565 & -2.429444 \\
\hline 1 & 2.321497 & 2.586904 & -2.211221 \\
\hline 6 & 3.055834 & 1.452886 & 2.683775 \\
\hline 1 & 2.094822 & 1.957338 & 2.545539 \\
\hline 6 & 6.293293 & -1.261079 & -0.035463 \\
\hline 1 & 5.887385 & -2.177453 & 0.418359 \\
\hline 6 & 2.646808 & 0.827200 & -3.372584 \\
\hline 1 & 1.861476 & 0.239815 & -2.890027 \\
\hline 1 & 2.234682 & 1.273884 & -4.282695 \\
\hline 1 & 3.454370 & 0.150344 & -3.671830 \\
\hline 6 & 4.253510 & 2.763570 & -3.098251 \\
\hline 1 & 4.587199 & 3.568566 & -2.437127 \\
\hline 1 & 5.125827 & 2.150111 & -3.347397 \\
\hline 1 & 3.880753 & 3.209944 & -4.025356 \\
\hline 6 & 2.792433 & 0.228868 & 3.567336 \\
\hline 1 & 3.719289 & -0.287425 & 3.838956 \\
\hline 1 & 2.316403 & 0.546837 & 4.500236 \\
\hline 1 & 2.124548 & -0.470021 & 3.060142 \\
\hline 6 & 4.013741 & 2.413254 & 3.402039 \\
\hline 1 & 3.615217 & 2.697999 & 4.380869 \\
\hline 1 & 4.985204 & 1.930635 & 3.556520 \\
\hline 1 & 4.186538 & 3.324790 & 2.824170 \\
\hline 6 & 7.476496 & -0.790616 & 0.822633 \\
\hline 1 & 7.902416 & 0.125749 & 0.401062 \\
\hline 1 & 7.177796 & -0.576362 & 1.851314 \\
\hline 1 & 8.261667 & -1.552462 & 0.848104 \\
\hline 6 & 6.792295 & -1.609312 & -1.436553 \\
\hline 1 & 7.560502 & -2.385481 & -1.377635 \\
\hline 1 & 5.990058 & -1.975644 & -2.081905 \\
\hline 1 & 7.240751 & -0.732067 & -1.915712 \\
\hline 6 & -3.324960 & -1.355373 & -2.364459 \\
\hline 1 & -2.708374 & -0.452566 & -2.410412 \\
\hline 6 & -3.855571 & 0.246316 & 2.48237 \\
\hline 1 & -3.559574 & 1.191960 & 2.01923 \\
\hline 6 & -4.812431 & -4.531209 & 1.297767 \\
\hline 1 & -4.339093 & -4.703268 & 2.274505 \\
\hline 6 & -6.333219 & -4.556949 & 1.504622 \\
\hline 1 & -6.659783 & -5.528778 & 1.88761 \\
\hline 1 & -6.650494 & -3.782741 & 2.20824 \\
\hline 1 & -6.842194 & -4.374348 & 0.55250 \\
\hline 6 & -4.387287 & -5.662554 & 0.362189 \\
\hline 1 & -4.930560 & -5.613307 & -0.58720 \\
\hline
\end{tabular}




\begin{tabular}{|c|c|c|c|}
\hline 1 & -3.316752 & -5.626831 & 0.138519 \\
\hline 1 & -4.611680 & -6.631519 & 0.816555 \\
\hline 6 & -2.782207 & -0.118323 & 3.514978 \\
\hline 1 & -3.044816 & -1.040298 & 4.045346 \\
\hline 1 & -1.811660 & -0.258184 & 3.031093 \\
\hline 1 & -2.683476 & 0.677184 & 4.259966 \\
\hline 6 & -5.215990 & 0.471313 & 3.152401 \\
\hline 1 & -5.973333 & 0.756841 & 2.416763 \\
\hline 1 & -5.561986 & -0.437213 & 3.656261 \\
\hline 1 & -5.146440 & 1.264722 & 3.902714 \\
\hline 6 & -4.570102 & -1.149400 & -3.236661 \\
\hline 1 & -4.283926 & -1.012351 & -4.284282 \\
\hline 1 & -5.225468 & -2.025395 & -3.175918 \\
\hline 1 & -5.148163 & -0.275937 & -2.926973 \\
\hline 6 & -2.487560 & -2.507411 & -2.933406 \\
\hline 1 & -2.033875 & -2.200520 & -3.880307 \\
\hline 1 & -1.688576 & -2.791715 & -2.246499 \\
\hline 1 & -3.103609 & -3.390000 & -3.138125 \\
\hline 6 & -0.890400 & -3.311825 & 1.125076 \\
\hline 6 & 0.114306 & -3.290465 & 2.278739 \\
\hline 1 & -0.292658 & -3.765215 & 3.174945 \\
\hline 1 & 0.383308 & -2.259592 & 2.526962 \\
\hline 6 & 1.338207 & -4.042989 & 1.762549 \\
\hline 6 & 1.614880 & -3.555997 & 0.346645 \\
\hline 1 & 1.709600 & -1.594999 & 0.661381 \\
\hline 8 & 2.159153 & -2.385163 & 0.148261 \\
\hline 1 & -1.554177 & -2.439378 & 1.119077 \\
\hline 1 & -1.506078 & -4.216518 & 1.139591 \\
\hline 8 & 2.026597 & -4.530703 & -0.445685 \\
\hline 6 & 2.484275 & -4.282463 & -1.817936 \\
\hline 6 & 1.432208 & -3.506516 & -2.602006 \\
\hline 1 & 1.285761 & -2.499188 & -2.205850 \\
\hline 1 & 1.766611 & -3.417496 & -3.639782 \\
\hline 1 & 0.476545 & -4.037288 & -2.587792 \\
\hline 6 & 2.629525 & -5.698205 & -2.364073 \\
\hline 1 & 2.954903 & -5.662240 & -3.406667 \\
\hline 1 & 3.369749 & -6.256306 & -1.784550 \\
\hline 1 & 1.673364 & -6.224167 & -2.310146 \\
\hline 6 & 3.836309 & -3.572453 & -1.812626 \\
\hline 1 & 4.514102 & -4.056401 & -1.101879 \\
\hline 1 & 4.277585 & -3.652871 & -2.811400 \\
\hline 1 & 3.740263 & -2.518114 & -1.548849 \\
\hline 6 & 2.591755 & -3.864151 & 2.615655 \\
\hline 1 & 2.423658 & -4.256481 & 3.621218 \\
\hline 1 & 3.439129 & -4.395518 & 2.173310 \\
\hline 1 & 2.854335 & -2.805307 & 2.695867 \\
\hline 1 & 1.097080 & -5.107132 & 1.663463 \\
\hline
\end{tabular}

Number of imaginary frequencies= The smallest frequency is= 1 $-184.4233 \mathrm{~cm}(-1)$

Electronic energy= Zero-point correction= Thermal correction to Energy= Thermal correction to Enthalpy= Thermal correction to Gibbs Free Energy=
$-3160.4374722 \quad\left(\mathbf{E}_{\mathrm{sol}}=\mathbf{- 3 1 6 0 . 4 8 8 2 1 2}\right)$

1.229710 (Hartree/Particle)

1.296391

1.297335

1.130683 


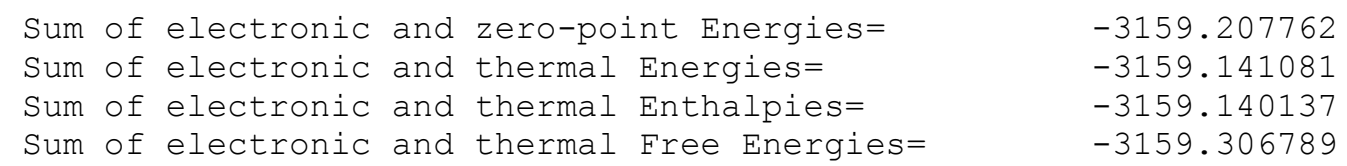

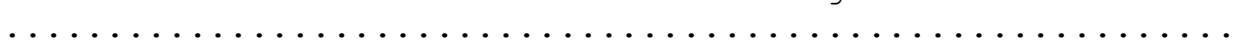

Cartesian Coordinates

\begin{tabular}{|c|c|c|c|}
\hline 6 & 2.879535 & 3.391235 & 0.854997 \\
\hline 6 & 2.483410 & 2.203267 & 0.290057 \\
\hline 6 & 1.129844 & 2.099122 & -0.121554 \\
\hline 6 & 0.194661 & 3.100898 & 0.053324 \\
\hline 6 & 0.598022 & 4.290137 & 0.747027 \\
\hline 1 & 3.919016 & 3.516722 & 1.146837 \\
\hline 6 & -3.269303 & 3.579905 & -1.557596 \\
\hline 6 & -3.902644 & 2.402043 & -1.083185 \\
\hline 6 & -3.218795 & 1.454788 & -0.363341 \\
\hline 6 & -1.868201 & 1.741477 & -0.031464 \\
\hline 6 & -1.205485 & 2.890131 & -0.408100 \\
\hline 6 & -1.899120 & 3.818300 & -1.253432 \\
\hline 1 & -4.946655 & 2.230577 & -1.333450 \\
\hline 6 & -1.972533 & 5.825021 & -2.619957 \\
\hline 6 & -1.268418 & 4.954909 & -1.827101 \\
\hline 1 & -1.470499 & 6.683660 & -3.053942 \\
\hline 1 & -0.214400 & 5.124572 & -1.636328 \\
\hline 6 & -3.345084 & 5.609956 & -2.884873 \\
\hline 6 & -3.973897 & 4.507851 & -2.368499 \\
\hline 1 & -3.891663 & 6.310161 & -3.508026 \\
\hline 1 & -5.021828 & 4.315641 & -2.581876 \\
\hline 6 & 0.119304 & 6.440995 & 1.770841 \\
\hline 6 & -0.314782 & 5.314017 & 1.120844 \\
\hline 1 & -0.595891 & 7.207175 & 2.052512 \\
\hline 1 & -1.368257 & 5.191453 & 0.896734 \\
\hline 6 & 1.487297 & 6.611435 & 2.087514 \\
\hline 6 & 2.385864 & 5.628386 & 1.769282 \\
\hline 1 & 1.816133 & 7.511643 & 2.596384 \\
\hline 1 & 3.436252 & 5.730037 & 2.028286 \\
\hline 8 & -1.209206 & 0.816799 & 0.757341 \\
\hline 8 & 0.763362 & 0.938226 & -0.776418 \\
\hline 15 & -0.140173 & -0.148452 & 0.015559 \\
\hline 8 & -0.802541 & -1.009046 & -1.030069 \\
\hline 8 & 0.628750 & -0.843596 & 1.110894 \\
\hline 1 & 0.374691 & -2.231826 & 1.118321 \\
\hline 8 & 0.036042 & -3.233943 & 1.084982 \\
\hline 6 & -1.388752 & -3.256293 & 1.281967 \\
\hline 6 & -1.991172 & -3.801719 & -0.012225 \\
\hline 6 & -0.931565 & -4.739015 & -0.577442 \\
\hline 6 & 0.395491 & -4.000649 & -0.480295 \\
\hline 8 & 0.640700 & -2.998091 & -1.289613 \\
\hline 1 & -1.591980 & -3.911521 & 2.134739 \\
\hline 1 & -1.744475 & -2.245579 & 1.509585 \\
\hline 6 & 1.965056 & 4.442689 & 1.110762 \\
\hline 8 & 1.405043 & -4.820326 & -0.248127 \\
\hline 6 & 2.804317 & -4.374608 & -0.292704 \\
\hline 6 & 3.229064 & -4.123855 & -1.737912 \\
\hline 1 & 2.875936 & -4.940216 & -2.375137 \\
\hline 1 & 2.837436 & -3.180551 & -2.116583 \\
\hline 1 & 4.320835 & -4.096920 & -1.790075 \\
\hline 6 & 3.534317 & -5.587950 & 0.272667 \\
\hline
\end{tabular}




\begin{tabular}{|c|c|c|c|}
\hline 1 & 4.610833 & -5.399468 & 0.291198 \\
\hline 1 & 3.195377 & -5.794533 & 1.290748 \\
\hline 1 & 3.343593 & -6.468935 & -0.346061 \\
\hline 0 & 3.011974 & -3.158278 & 0.600996 \\
\hline & 2.546848 & -2.254071 & 0.198538 \\
\hline & 2.615470 & -3.351406 & 1.602178 \\
\hline & 4.083511 & -2.959600 & 0.689265 \\
\hline 1 & -0.055794 & -2.224928 & -1.256810 \\
\hline 6 & 3.461367 & 1.077283 & 0.169079 \\
\hline 6 & 3.956860 & 0.671584 & -1.085018 \\
\hline & 3.977469 & 0.502621 & 1.347734 \\
\hline 6 & 5.031607 & -0.217765 & -1.130739 \\
\hline 6 & 5.075362 & -0.355169 & 1.250469 \\
\hline 6 & 5.640951 & -0.700344 & 0.026906 \\
\hline 1 & 5.430770 & -0.504921 & -2.101961 \\
\hline & 5.508654 & -0.763965 & 2.161575 \\
\hline 6 & -3.830755 & 0.150042 & 0.031823 \\
\hline 6 & -4.025826 & -0.162975 & 1.391359 \\
\hline 6 & -4.206808 & -0.774488 & -0.965509 \\
\hline 6 & -4.610682 & -1.384872 & 1.729902 \\
\hline 6 & -4.805019 & -1.972900 & -0.574058 \\
\hline 6 & -5.010577 & -2.303897 & 0.762931 \\
\hline 1 & -4.764591 & -1.614164 & 2.781667 \\
\hline & -5.105840 & -2.687684 & -1.338839 \\
\hline 6 & -5.698326 & -3.606511 & 1.126961 \\
\hline 1 & -5.541394 & -4.298512 & 0.288005 \\
\hline 6 & -3.665769 & 0.795492 & 2.516975 \\
\hline 1 & -3.191220 & 1.679747 & 2.083666 \\
\hline 6 & 3.381352 & 1.185380 & -2.395253 \\
\hline 1 & 2.596468 & 1.911976 & -2.169029 \\
\hline 6 & 3.375781 & 0.764190 & 2.722506 \\
\hline 1 & 2.449933 & 1.331066 & 2.586766 \\
\hline 6 & 6.910419 & -1.530432 & -0.036150 \\
\hline 1 & 7.170751 & -1.804511 & 0.994224 \\
\hline 5 & -3.996256 & -0.527552 & -2.454060 \\
\hline & -3.386034 & 0.371545 & -2.570920 \\
\hline 6 & -5.339899 & -0.289910 & -3.155732 \\
\hline 1 & -5.892933 & 0.536381 & -2.699776 \\
\hline 1 & -5.185606 & -0.058553 & -4.214046 \\
\hline 1 & -5.971643 & -1.182939 & -3.094627 \\
\hline 6 & -3.234231 & -1.672518 & -3.132086 \\
\hline 1 & -3.780379 & -2.620132 & -3.067464 \\
\hline 1 & -3.093818 & -1.447108 & -4.193912 \\
\hline 1 & -2.250520 & -1.794181 & -2.673943 \\
\hline 6 & -5.130600 & -4.260400 & 2.388595 \\
\hline 1 & -5.567103 & -5.253078 & 2.530726 \\
\hline 1 & -4.042769 & -4.367270 & 2.332520 \\
\hline 1 & -5.364397 & -3.671594 & 3.281014 \\
\hline 6 & -7.209155 & -3.381321 & 1.272708 \\
\hline 1 & -7.406535 & -2.675826 & 2.086745 \\
\hline 1 & -7.633290 & -2.963130 & 0.356048 \\
\hline 1 & -7.725301 & -4.319481 & 1.499292 \\
\hline 6 & 6.738461 & -2.824162 & -0.836592 \\
\hline 1 & 7.680051 & -3.380484 & -0.876447 \\
\hline 1 & 5.980839 & -3.471494 & -0.385594 \\
\hline 1 & 6.431411 & -2.611733 & -1.866283 \\
\hline 5 & 8.063726 & -0.696726 & -0.608094 \\
\hline & 7.855921 & -0.416167 & -1.646 \\
\hline
\end{tabular}




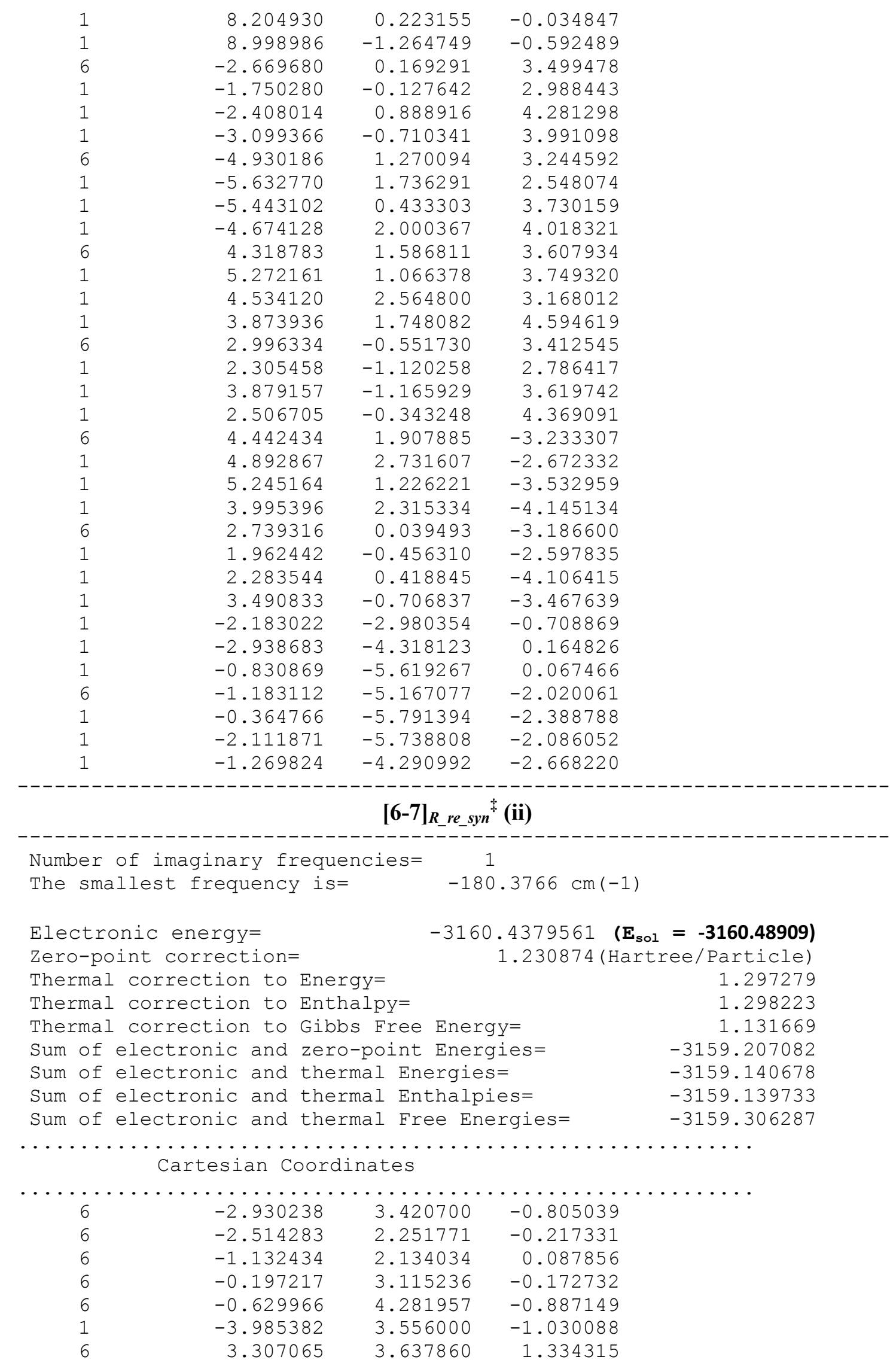




\begin{tabular}{|c|c|c|c|}
\hline 6 & 3.919989 & 2.429572 & 0.913444 \\
\hline 6 & 3.215187 & 1.457131 & 0.250104 \\
\hline 6 & 1.863764 & 1.742186 & -0.079360 \\
\hline 6 & 1.216963 & 2.914480 & 0.251326 \\
\hline 6 & 1.934745 & 3.876307 & 1.038763 \\
\hline 1 & 4.965440 & 2.257774 & 1.157276 \\
\hline 6 & 2.055176 & 5.949725 & 2.299798 \\
\hline 6 & 1.327523 & 5.048452 & 1.565072 \\
\hline 1 & 1.570032 & 6.835684 & 2.696508 \\
\hline 1 & 0.272752 & 5.222729 & 1.384778 \\
\hline 6 & 3.429401 & 5.732651 & 2.553070 \\
\hline 6 & 4.036091 & 4.597458 & 2.084545 \\
\hline 1 & 3.994050 & 6.457699 & 3.129964 \\
\hline 1 & 5.085066 & 4.402886 & 2.290525 \\
\hline 6 & -0.185244 & 6.380201 & -2.028272 \\
\hline 6 & 0.271838 & 5.275314 & -1.356455 \\
\hline 1 & 0.521498 & 7.122405 & -2.385129 \\
\hline 1 & 1.334767 & 5.144816 & -1.186699 \\
\hline 6 & -1.567442 & 6.557231 & -2.270312 \\
\hline 6 & -2.458357 & 5.603221 & -1.855657 \\
\hline 1 & -1.914745 & 7.439740 & -2.797618 \\
\hline 1 & -3.521009 & 5.712106 & -2.054440 \\
\hline 8 & 1.194327 & 0.791412 & -0.824474 \\
\hline 8 & -0.740921 & 0.983989 & 0.744824 \\
\hline 15 & 0.098531 & -0.133983 & -0.069277 \\
\hline 8 & 0.726074 & -1.030552 & 0.967708 \\
\hline 8 & -0.701416 & -0.791702 & -1.165709 \\
\hline 1 & -0.398633 & -2.178728 & -1.267069 \\
\hline 8 & -0.042649 & -3.171684 & -1.275743 \\
\hline 6 & 1.388943 & -3.161980 & -1.404043 \\
\hline 6 & 1.946874 & -3.739083 & -0.103405 \\
\hline 6 & 0.875677 & -4.692520 & 0.413100 \\
\hline 6 & -0.464495 & -3.986871 & 0.262261 \\
\hline 8 & -0.781993 & -3.002851 & 1.067213 \\
\hline 1 & 1.647735 & -3.779927 & -2.268984 \\
\hline 1 & 1.737320 & -2.138360 & -1.576772 \\
\hline 6 & -2.015474 & 4.440059 & -1.172133 \\
\hline 8 & -1.436216 & -4.828286 & -0.040624 \\
\hline 6 & -2.842165 & -4.413328 & -0.141431 \\
\hline 6 & -3.422850 & -4.126024 & 1.241011 \\
\hline 1 & -3.139843 & -4.918218 & 1.940443 \\
\hline 1 & -3.087333 & -3.164570 & 1.629374 \\
\hline 1 & -4.515018 & -4.111073 & 1.163185 \\
\hline 6 & -3.492124 & -5.653727 & -0.743903 \\
\hline 1 & -4.562196 & -5.479603 & -0.883833 \\
\hline 1 & -3.042657 & -5.884711 & -1.712729 \\
\hline 1 & -3.360556 & -6.513521 & -0.081477 \\
\hline 6 & -2.977311 & -3.221593 & -1.078058 \\
\hline 1 & -2.564121 & -2.307029 & -0.644272 \\
\hline 1 & -2.484229 & -3.425551 & -2.032651 \\
\hline 1 & -4.038370 & -3.039265 & -1.268340 \\
\hline 1 & -0.075193 & -2.242898 & 1.118991 \\
\hline 6 & -3.467588 & 1.136822 & 0.071248 \\
\hline 6 & -3.732459 & 0.748771 & 1.401897 \\
\hline 6 & -4.135121 & 0.496322 & -0.990891 \\
\hline 6 & -4.688923 & -0.235739 & 1.635739 \\
\hline 6 & -5.107220 & -0.467475 & -0.699451 \\
\hline 6 & -5.407844 & -0.842161 & 0.605443 \\
\hline
\end{tabular}




\begin{tabular}{|c|c|c|c|}
\hline L & -4.904192 & -0.529464 & 2.662393 \\
\hline[ & -5.628359 & -0.941086 & -1.527793 \\
\hline 6 & 3.813298 & 0.130311 & -0.088849 \\
\hline 6 & 4.004778 & -0.243224 & -1.436029 \\
\hline 6 & 4.190621 & -0.748917 & 0.943780 \\
\hline & 4.594289 & -1.474204 & -1.714769 \\
\hline 6 & 4.810538 & -1.957958 & 0.610470 \\
\hline 6 & 5.021714 & -2.342662 & -0.708561 \\
\hline & 4.740770 & -1.765331 & -2.754268 \\
\hline & 5.112274 & -2.620855 & 1.416395 \\
\hline & 5.706803 & -3.644043 & -1.094957 \\
\hline 1 & 5.075225 & -4.123155 & -1.85771 \\
\hline 6 & 3.640864 & 0.666131 & -2.600914 \\
\hline 1 & 3.172317 & 1.570152 & -2.202846 \\
\hline 6 & -3.056127 & 1.391709 & 2.603192 \\
\hline 1 & -2.327028 & 2.123127 & 2.245998 \\
\hline 6 & -3.840711 & 0.792298 & -2.456291 \\
\hline 1 & -3.007757 & 1.497882 & -2.505598 \\
\hline 6 & -6.521435 & -1.812649 & 0.961564 \\
\hline 1 & -6.156177 & -2.428765 & 1.794984 \\
\hline 6 & 3.952864 & -0.450967 & 2.418790 \\
\hline 1 & 3.339376 & 0.450726 & 2.492364 \\
\hline 6 & 5.278910 & -0.188448 & 3.14353 \\
\hline 1 & 5.837879 & 0.628725 & 2.678549 \\
\hline 1 & 5.099045 & 0.070577 & 4.19135 \\
\hline 7 & 5.916135 & -1.079321 & 3.12213 \\
\hline 6 & 3.175017 & -1.576798 & 3.111728 \\
\hline 1 & 3.725773 & -2.523790 & 3.08967 \\
\hline 1 & 3.005500 & -1.319875 & 4.162133 \\
\hline 1 & 2.204614 & -1.715925 & 2.630344 \\
\hline 6 & 7.071872 & -3.354141 & -1.735265 \\
\hline 1 & 7.548995 & -4.280312 & -2.070122 \\
\hline 1 & 6.978368 & -2.684831 & -2.594129 \\
\hline 1 & 7.730750 & -2.872412 & -1.005562 \\
\hline 6 & 5.876919 & -4.627092 & 0.062672 \\
\hline 1 & 6.578589 & -4.234753 & 0.806319 \\
\hline 1 & 4.930577 & -4.834821 & 0.571837 \\
\hline 1 & 6.281776 & -5.573648 & -0.305258 \\
\hline 6 & -7.743334 & -1.029053 & 1.463431 \\
\hline 1 & -8.543131 & -1.708390 & 1.774550 \\
\hline 1 & -7.481669 & -0.390482 & 2.311194 \\
\hline 1 & -8.128461 & -0.385836 & 0.665282 \\
\hline 6 & -6.931875 & -2.747701 & -0.174265 \\
\hline 1 & -7.395276 & -2.192049 & -0.996103 \\
\hline 1 & -6.077127 & -3.298962 & -0.57830 \\
\hline 1 & -7.666575 & -3.474433 & 0.183894 \\
\hline 6 & 2.636331 & 0.007089 & -3.552579 \\
\hline 1 & 1.704223 & -0.233614 & -3.034723 \\
\hline 1 & 2.398892 & 0.685175 & -4.37803 \\
\hline 1 & 3.046037 & -0.911332 & -3.98759 \\
\hline 6 & 4.904899 & 1.101229 & -3.353927 \\
\hline 1 & 5.616283 & 1.586502 & -2.679673 \\
\hline 1 & 5.406977 & 0.241391 & -3.810104 \\
\hline 1 & 4.651184 & 1.804060 & -4.153359 \\
\hline 6 & -5.049435 & 1.435651 & -3.145975 \\
\hline 7 & -5.908332 & 0.756085 & -3.142760 \\
\hline$\perp$ & -5.356152 & 2.357573 & -2.64296 \\
\hline 1 & -4.814701 & 1.674213 & -4.18777 \\
\hline
\end{tabular}




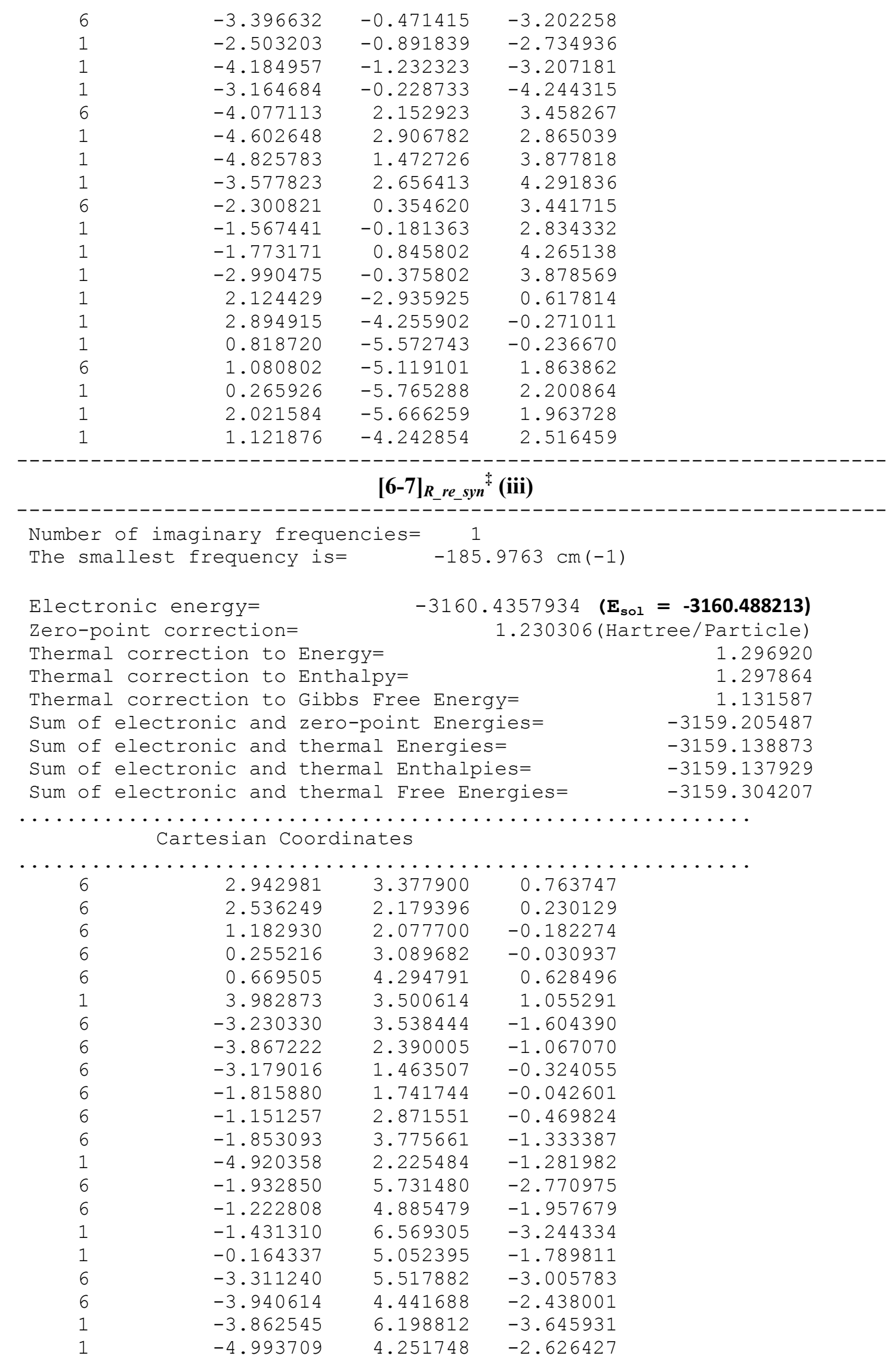




\begin{tabular}{|c|c|c|c|}
\hline 6 & 0.211651 & 6.478424 & 1.591237 \\
\hline 6 & -0.233325 & 5.337458 & 0.973617 \\
\hline 1 & -0.496338 & 7.259070 & 1.850446 \\
\hline 1 & -1.288093 & 5.219129 & 0.753733 \\
\hline 6 & 1.581210 & 6.645156 & 1.902604 \\
\hline 6 & 2.470295 & 5.644974 & 1.612119 \\
\hline 1 & 1.919007 & 7.556537 & 2.385041 \\
\hline 1 & 3.521732 & 5.744031 & 1.867866 \\
\hline 8 & -1.145869 & 0.831111 & 0.752650 \\
\hline 8 & 0.808039 & 0.905551 & -0.811279 \\
\hline 15 & -0.106501 & -0.157638 & -0.000716 \\
\hline 8 & -0.800173 & -1.009293 & -1.033275 \\
\hline 8 & 0.662041 & -0.860964 & 1.089564 \\
\hline 1 & 0.362016 & -2.238911 & 1.121388 \\
\hline 8 & -0.001300 & -3.232679 & 1.091814 \\
\hline 6 & -1.431303 & -3.221483 & 1.257012 \\
\hline 6 & -2.015325 & -3.757874 & -0.048394 \\
\hline 6 & -0.962942 & -4.715517 & -0.592268 \\
\hline 6 & 0.374448 & -4.002508 & -0.466931 \\
\hline 8 & 0.650254 & -3.001992 & -1.270272 \\
\hline 1 & -1.669727 & -3.866285 & 2.108288 \\
\hline 1 & -1.770324 & -2.201490 & 1.470372 \\
\hline 6 & 2.038207 & 4.445126 & 0.987649 \\
\hline 8 & 1.366906 & -4.838168 & -0.219164 \\
\hline 6 & 2.774242 & -4.416138 & -0.244387 \\
\hline 6 & 3.222447 & -4.172457 & -1.683854 \\
\hline 1 & 2.859382 & -4.979924 & -2.326817 \\
\hline 1 & 2.856519 & -3.220218 & -2.065849 \\
\hline 1 & 4.315085 & -4.169481 & -1.722801 \\
\hline 6 & 3.476158 & -5.641724 & 0.329915 \\
\hline 1 & 4.555171 & -5.470244 & 0.364077 \\
\hline 1 & 3.119216 & -5.843703 & 1.342740 \\
\hline 1 & 3.280347 & -6.518961 & -0.292536 \\
\hline 6 & 2.990217 & -3.203423 & 0.652203 \\
\hline 1 & 2.543837 & -2.292331 & 0.244511 \\
\hline 1 & 2.578524 & -3.390579 & 1.648474 \\
\hline 1 & 4.063700 & -3.021732 & 0.753850 \\
\hline 1 & -0.046491 & -2.231133 & -1.258442 \\
\hline 6 & 3.500271 & 1.038170 & 0.145544 \\
\hline 6 & 4.008797 & 0.602280 & -1.092665 \\
\hline 6 & 3.987589 & 0.477224 & 1.342745 \\
\hline 6 & 5.070694 & -0.303537 & -1.105331 \\
\hline 6 & 5.073924 & -0.398066 & 1.278792 \\
\hline 6 & 5.653557 & -0.774158 & 0.070823 \\
\hline 1 & 5.480837 & -0.614391 & -2.064678 \\
\hline 1 & 5.486537 & -0.796238 & 2.204056 \\
\hline 6 & -3.816828 & 0.206369 & 0.174312 \\
\hline 6 & -3.976745 & -0.002785 & 1.558439 \\
\hline 6 & -4.293347 & -0.756393 & -0.742964 \\
\hline 6 & -4.657444 & -1.141231 & 1.995209 \\
\hline 6 & -4.965723 & -1.877866 & -0.252483 \\
\hline 6 & -5.182344 & -2.078688 & 1.110551 \\
\hline 1 & -4.814086 & -1.285176 & 3.062622 \\
\hline 1 & -5.353778 & -2.605021 & -0.963988 \\
\hline 6 & -6.023534 & -3.235693 & 1.619454 \\
\hline 1 & -6.089091 & -3.130491 & 2.709710 \\
\hline 6 & -3.498823 & 0.989615 & 2.608957 \\
\hline 1 & -2.981895 & 1.811641 & 2.108128 \\
\hline
\end{tabular}




\begin{tabular}{|c|c|c|c|}
\hline 6 & 3.457868 & 1.100416 & -2.419304 \\
\hline 1 & 2.677312 & 1.838428 & -2.215644 \\
\hline 6 & 3.365998 & 0.773677 & 2.701249 \\
\hline 1 & 2.441832 & 1.335965 & 2.536465 \\
\hline 6 & 6.911106 & -1.624189 & 0.044580 \\
\hline 1 & 7.144240 & -1.891084 & 1.083327 \\
\hline 6 & -4.155221 & -0.602500 & -2.253581 \\
\hline 1 & -3.546215 & 0.281588 & -2.456065 \\
\hline 6 & -5.532680 & -0.388792 & -2.896194 \\
\hline & -6.059336 & 0.458111 & -2.446469 \\
\hline & -5.430874 & -0.201267 & -3.969382 \\
\hline & -6.164679 & -1.274362 & -2.767742 \\
\hline 6 & -3.437000 & -1.790073 & -2.904820 \\
\hline 1 & -3.933590 & -2.741018 & -2.679732 \\
\hline 1 & -3.431379 & -1.669383 & -3.992761 \\
\hline 1 & -2.401659 & -1.837115 & -2.560670 \\
\hline 6 & -7.447293 & -3.164180 & 1.05499 \\
\hline 1 & -8.073657 & -3.951203 & 1.485845 \\
\hline 1 & -7.907581 & -2.196565 & 1.270358 \\
\hline 1 & -7.440860 & -3.298404 & -0.031684 \\
\hline 6 & -5.396313 & -4.599546 & 1.319508 \\
\hline 1 & -5.279310 & -4.745625 & 0.23957 \\
\hline 1 & -4.411798 & -4.693978 & 1.787147 \\
\hline 1 & -6.030708 & -5.407313 & 1.697155 \\
\hline 6 & 6.738246 & -2.924031 & -0.745696 \\
\hline 1 & 7.670525 & -3.497067 & -0.752757 \\
\hline 1 & 5.956798 & -3.552359 & -0.308769 \\
\hline 1 & 6.464079 & -2.719216 & -1.786130 \\
\hline 6 & 8.089121 & -0.813449 & -0.509840 \\
\hline 1 & 7.906157 & -0.537074 & -1.553578 \\
\hline 1 & 8.233685 & 0.108268 & 0.059646 \\
\hline 1 & 9.014744 & -1.396053 & -0.471668 \\
\hline 6 & -2.504198 & 0.342111 & 3.578871 \\
\hline 1 & -1.641987 & -0.060731 & 3.041096 \\
\hline 1 & -2.141346 & 1.081827 & 4.298994 \\
\hline 1 & -2.976257 & -0.468107 & 4.144881 \\
\hline 6 & -4.688880 & 1.598096 & 3.361618 \\
\hline 1 & -5.385011 & 2.080849 & 2.669750 \\
\hline 1 & -5.241209 & 0.831420 & 3.914568 \\
\hline 1 & -4.342404 & 2.346236 & 4.081114 \\
\hline 6 & 4.296089 & 1.619663 & 3.578301 \\
\hline 1 & 5.249010 & 1.104821 & 3.741770 \\
\hline 1 & 4.513636 & 2.588050 & 3.118788 \\
\hline 1 & 3.839778 & 1.801834 & 4.556123 \\
\hline 6 & 2.976754 & -0.523465 & 3.421055 \\
\hline 1 & 2.303705 & -1.114271 & 2.796211 \\
\hline 1 & 3.857235 & -1.126249 & 3.668186 \\
\hline 1 & 2.463336 & -0.288783 & 4.358887 \\
\hline 6 & 4.538985 & 1.798089 & -3.252636 \\
\hline 1 & 4.987095 & 2.628180 & -2.699205 \\
\hline 1 & 5.340480 & 1.103752 & -3.524874 \\
\hline 1 & 4.111413 & 2.192206 & -4.179518 \\
\hline 6 & 2.814320 & -0.051183 & -3.200846 \\
\hline 1 & 2.023329 & -0.527505 & -2.614629 \\
\hline 1 & 2.376479 & 0.317555 & -4.133587 \\
\hline 1 & 3.560666 & -0.811184 & -3.457837 \\
\hline 1 & -2.177112 & -2.933229 & -0.746098 \\
\hline 1 & -2.973296 & -4.256029 & 0.10732 \\
\hline
\end{tabular}




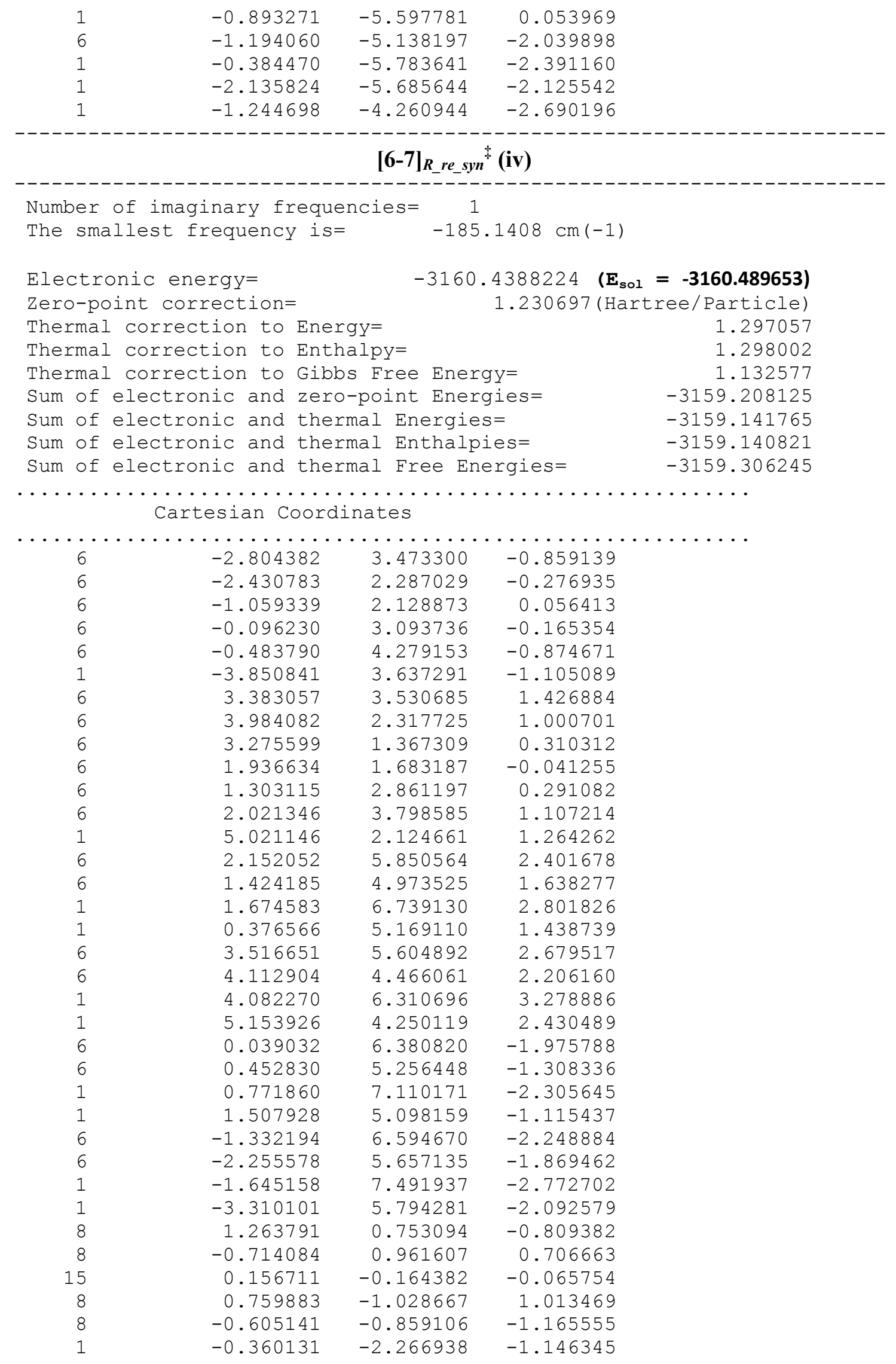




\begin{tabular}{|c|c|c|c|}
\hline 8 & -0.064876 & -3.273772 & -1.062371 \\
\hline 6 & 1.367369 & -3.366049 & -1.137779 \\
\hline 6 & 1.840668 & -3.879074 & 0.222861 \\
\hline 6 & 0.693372 & -4.726783 & 0.760875 \\
\hline 6 & -0.591004 & -3.948487 & 0.515906 \\
\hline 8 & -0.860416 & -2.889662 & 1.238848 \\
\hline 1 & 1.606614 & -4.064777 & -1.945236 \\
\hline 1 & 1.790313 & -2.383271 & -1.373845 \\
\hline 6 & -1.857445 & 4.474605 & -1.191360 \\
\hline 8 & -1.608137 & -4.741133 & 0.236159 \\
\hline 6 & -2.980225 & -4.242078 & 0.062316 \\
\hline 6 & -3.581148 & -3.836060 & 1.405040 \\
\hline 1 & -3.394419 & -4.612323 & 2.153046 \\
\hline 1 & -3.178653 & -2.886955 & 1.759229 \\
\hline 1 & -4.664369 & -3.730327 & 1.282141 \\
\hline 6 & -3.690411 & -5.473203 & -0.489043 \\
\hline 1 & -4.744926 & -5.245714 & -0.665725 \\
\hline 1 & -3.234334 & -5.784155 & -1.432069 \\
\hline 1 & -3.625229 & -6.301087 & 0.222037 \\
\hline 6 & -3.010671 & -3.101858 & -0.945339 \\
\hline 1 & -2.558034 & -2.188414 & -0.550248 \\
\hline 1 & -2.499455 & -3.390581 & -1.868051 \\
\hline 1 & -4.051691 & -2.869084 & -1.184030 \\
\hline 1 & -0.104899 & -2.173613 & 1.243960 \\
\hline 6 & -3.427620 & 1.201491 & -0.022752 \\
\hline 6 & -3.751036 & 0.817596 & 1.295354 \\
\hline 6 & -4.088963 & 0.596790 & -1.109857 \\
\hline 6 & -4.765107 & -0.117507 & 1.492497 \\
\hline 6 & -5.120706 & -0.313195 & -0.856279 \\
\hline 6 & -5.484535 & -0.675499 & 0.436564 \\
\hline 1 & -5.025656 & -0.407976 & 2.509500 \\
\hline 1 & -5.636736 & -0.758986 & -1.703300 \\
\hline 6 & 3.848086 & 0.032085 & -0.041091 \\
\hline 6 & 4.052421 & -0.319954 & -1.390932 \\
\hline 6 & 4.182509 & -0.877491 & 0.982692 \\
\hline 6 & 4.614489 & -1.561052 & -1.690328 \\
\hline 6 & 4.750108 & -2.103973 & 0.629064 \\
\hline 6 & 4.975389 & -2.467942 & -0.695187 \\
\hline 1 & 4.787612 & -1.817167 & -2.733397 \\
\hline 1 & 5.021745 & -2.805699 & 1.416176 \\
\hline 6 & 5.653001 & -3.783473 & -1.029506 \\
\hline 1 & 5.610443 & -4.408751 & -0.127630 \\
\hline 6 & 3.726142 & 0.617409 & -2.544064 \\
\hline 1 & 3.262668 & 1.520744 & -2.139039 \\
\hline 6 & -3.072344 & 1.409509 & 2.520896 \\
\hline 1 & -2.309580 & 2.119234 & 2.191005 \\
\hline 6 & -3.711549 & 0.865004 & -2.561064 \\
\hline 1 & -2.848152 & 1.534794 & -2.57440 \\
\hline 6 & -6.666040 & -1.579263 & 0.744983 \\
\hline 1 & -6.407856 & -2.146567 & 1.649660 \\
\hline 6 & 3.973954 & -0.580135 & 2.462476 \\
\hline 1 & 3.408166 & 0.350733 & 2.55028 \\
\hline 6 & 5.324660 & -0.384970 & 3.164185 \\
\hline 1 & 5.916033 & 0.402126 & 2.687241 \\
\hline 1 & 5.176770 & -0.116051 & 4.214542 \\
\hline 1 & 5.915488 & -1.306919 & 3.132343 \\
\hline 6 & 3.154385 & -1.667076 & 3.16780 \\
\hline 1 & 3.637917 & -2.648179 & 3.10189 \\
\hline
\end{tabular}




\begin{tabular}{|c|c|c|c|}
\hline & & & \\
\hline 1 & 3.049601 & -1.422915 & 4.229676 \\
\hline 1 & 2.156699 & -1.730277 & 2.728422 \\
\hline 6 & 4.960432 & -4.543470 & -2.163775 \\
\hline 1 & 5.438107 & -5.515295 & -2.318984 \\
\hline 1 & 3.902206 & -4.713808 & -1.945782 \\
\hline 1 & 5.023359 & -3.992999 & -3.107551 \\
\hline 6 & 7.130168 & -3.545103 & -1.368756 \\
\hline 1 & 7.215817 & -2.917602 & -2.262382 \\
\hline 1 & 7.642769 & -3.034007 & -0.549755 \\
\hline 1 & 7.643786 & -4.491238 & -1.565734 \\
\hline 6 & -7.897039 & -0.720856 & 1.070697 \\
\hline 1 & -8.749965 & -1.348072 & 1.348245 \\
\hline 1 & -7.689577 & -0.032137 & 1.893974 \\
\hline 1 & -8.178585 & -0.124381 & 0.196626 \\
\hline 6 & -7.000550 & -2.578798 & -0.361148 \\
\hline 1 & -7.361565 & -2.069685 & -1.260618 \\
\hline 1 & -6.131816 & -3.182587 & -0.641023 \\
\hline 1 & -7.793094 & -3.255971 & -0.029900 \\
\hline 6 & 2.729535 & -0.015624 & -3.521669 \\
\hline 1 & 1.803629 & -0.293365 & -3.011331 \\
\hline 1 & 2.481115 & 0.692141 & -4.318438 \\
\hline 1 & 3.152710 & -0.909032 & -3.993841 \\
\hline 6 & 5.007879 & 1.049593 & -3.267636 \\
\hline 1 & 5.710453 & 1.520928 & -2.574488 \\
\hline 1 & 5.511213 & 0.191209 & -3.724973 \\
\hline 1 & 4.775252 & 1.763970 & -4.063260 \\
\hline 6 & -4.853386 & 1.548923 & -3.321136 \\
\hline 1 & -5.740497 & 0.907654 & -3.357133 \\
\hline 1 & -5.144637 & 2.490351 & -2.845658 \\
\hline 1 & -4.553898 & 1.763359 & -4.351543 \\
\hline 6 & -3.281457 & -0.428545 & -3.263294 \\
\hline 1 & -2.429457 & -0.874682 & -2.744474 \\
\hline 1 & -4.098686 & -1.157794 & -3.293595 \\
\hline 1 & -2.988557 & -0.214995 & -4.296329 \\
\hline 6 & -4.075243 & 2.190527 & 3.379245 \\
\hline 1 & -4.570747 & 2.970342 & 2.793889 \\
\hline 1 & -4.849014 & 1.529480 & 3.783331 \\
\hline 1 & -3.566652 & 2.664528 & 4.224337 \\
\hline 6 & -2.371605 & 0.323265 & 3.344894 \\
\hline 1 & -1.644755 & -0.221278 & 2.737033 \\
\hline 1 & -1.844381 & 0.771900 & 4.192592 \\
\hline 1 & -3.096403 & -0.394112 & 3.745485 \\
\hline 1 & 2.038020 & -3.034770 & 0.888502 \\
\hline 1 & 2.762936 & -4.461015 & 0.140564 \\
\hline 1 & 0.600321 & -5.644174 & 0.169036 \\
\hline 6 & 0.817485 & -5.066050 & 2.243738 \\
\hline 1 & -0.053707 & -5.629686 & 2.588291 \\
\hline 1 & 1.711714 & -5.669202 & 2.417989 \\
\hline 1 & 0.896688 & -4.152309 & 2.838925 \\
\hline
\end{tabular}

Number of imaginary frequencies= 1

The smallest frequency is= $-221.5872 \mathrm{~cm}(-1)$

Electronic energy= Zero-point correction= Thermal correction to Energy=
$-3160.4345897\left(\boldsymbol{E}_{\text {sol }}=\mathbf{- 3 1 6 0 . 4 8 5 5 3 2}\right)$

1.228570 (Hartree/Particle)

1.295356 


\begin{tabular}{|c|c|c|c|c|}
\hline \multicolumn{5}{|c|}{ Thermal correction to Enthalpy= } \\
\hline Thermal & 1 correction to Gibl & s Free Ener & & 1.128847 \\
\hline Sum of & electronic and zerc & -point Ener & ies $=$ & -3159.206020 \\
\hline Sum of & electronic and the & mal Energie & & -3159.139234 \\
\hline Sum of & electronic and the & mal Enthalp & $\mathrm{es}=$ & -3159.138289 \\
\hline Sum of & electronic and the & mal Free Er & rgies= & -3159.305743 \\
\hline$\cdots \cdots$ & $\begin{array}{c} \\
\quad \text { Cartesian Coor }\end{array}$ & nates & $\ldots \ldots \ldots$ & \\
\hline$\ldots$ & $\ldots \ldots$ & $\ldots \ldots$ & $\cdots \cdots \cdots$ & \\
\hline 6 & -2.299895 & 4.176009 & -1.555960 & \\
\hline 6 & -3.160696 & 3.102542 & -1.206547 & \\
\hline 6 & -2.703113 & 2.006304 & -0.520480 & \\
\hline 6 & -1.351973 & 2.030212 & -0.086673 & \\
\hline 6 & -0.476486 & 3.068327 & -0.328649 & \\
\hline 6 & -0.936404 & 4.152841 & -1.147059 & \\
\hline 1 & -4.203532 & 3.147349 & -1.508138 & \\
\hline 6 & 2.812051 & 3.915967 & 1.485986 & \\
\hline 6 & 3.547868 & 2.741563 & 1.182350 & \\
\hline 6 & 2.987917 & 1.695740 & 0.491261 & \\
\hline 6 & 1.661720 & 1.869665 & 0.017621 & \\
\hline 6 & 0.904076 & 3.002490 & 0.229183 & \\
\hline 6 & 1.467440 & 4.045388 & 1.036672 & \\
\hline 1 & 4.575621 & 2.662685 & 1.526640 & \\
\hline 6 & 1.310953 & 6.169075 & 2.205550 & \\
\hline 6 & 0.729274 & 5.191096 & 1.439554 & \\
\hline 1 & 0.727688 & 7.033499 & 2.505918 & \\
\hline 1 & -0.309715 & 5.281408 & 1.143354 & \\
\hline 6 & 2.660183 & 6.058652 & 2.614827 & \\
\hline 6 & 3.388784 & 4.952518 & 2.265977 & \\
\hline 1 & 3.108726 & 6.842941 & 3.215725 & \\
\hline 1 & 4.419269 & 4.841324 & 2.592150 & \\
\hline 6 & -0.563633 & 6.219966 & -2.366689 & \\
\hline 6 & -0.079970 & 5.194692 & -1.594298 & \\
\hline 1 & 0.108877 & 7.001904 & -2.704282 & \\
\hline 1 & 0.970419 & 5.165753 & -1.325990 & \\
\hline 6 & -1.928403 & 6.264144 & -2.734859 & \\
\hline 6 & -2.772967 & 5.260081 & -2.340420 & \\
\hline 1 & -2.297907 & 7.084704 & -3.341089 & \\
\hline 1 & -3.819034 & 5.266833 & -2.634496 & \\
\hline 8 & 1.130243 & 0.841685 & -0.737014 & \\
\hline 8 & -0.934108 & 0.958495 & 0.680036 & \\
\hline 15 & 0.049278 & -0.122150 & -0.010424 & \\
\hline 8 & 0.645785 & -0.909970 & 1.125235 & \\
\hline 8 & -0.607936 & -0.871718 & -1.141884 & \\
\hline 1 & 0.746905 & -2.257467 & 0.779706 & \\
\hline 6 & 3.745116 & 0.426320 & 0.255390 & \\
\hline 6 & 4.072480 & -0.400287 & 1.349689 & \\
\hline 6 & 4.182943 & 0.081980 & -1.039441 & \\
\hline 6 & 4.933677 & -1.480749 & 1.141433 & \\
\hline 6 & 5.012825 & -1.028633 & -1.199484 & \\
\hline 6 & 5.433618 & -1.800585 & -0.116863 & \\
\hline 6 & -3.580162 & 0.833311 & -0.210280 & \\
\hline 6 & -3.941148 & -0.065209 & -1.234835 & \\
\hline 6 & -4.057369 & 0.640544 & 1.098726 & \\
\hline 6 & -4.836354 & -1.093944 & -0.934994 & \\
\hline 6 & -4.954455 & -0.400251 & 1.346986 & \\
\hline 6 & -5.371189 & -1.267389 & 0.340428 & \\
\hline 8 & 0.965275 & -3.226783 & 0.426041 & \\
\hline
\end{tabular}




\begin{tabular}{|c|c|c|c|}
\hline 1 & -5.337492 & -0.527908 & 2.356874 \\
\hline 1 & -5.134746 & -1.785345 & -1.720640 \\
\hline 1 & 5.360735 & -1.281336 & -2.199513 \\
\hline 1 & 5.219061 & -2.098756 & 1.990290 \\
\hline 6 & -3.636033 & 1.527581 & 2.259673 \\
\hline 1 & -2.938362 & 2.282103 & 1.884981 \\
\hline 6 & -3.395065 & 0.059255 & -2.650310 \\
\hline$\perp$ & -2.473381 & 0.647812 & -2.600435 \\
\hline 6 & -6.425637 & -2.331441 & 0.583929 \\
\hline 1 & -6.299408 & -3.096150 & -0.194018 \\
\hline 6 & -2.901583 & 0.711571 & 3.330198 \\
\hline 1 & -2.036695 & 0.199090 & 2.900706 \\
\hline 1 & -2.551335 & 1.365969 & 4.134541 \\
\hline 1 & -3.565199 & -0.038019 & 3.775058 \\
\hline 6 & -4.836184 & 2.272452 & 2.855055 \\
\hline 1 & -5.345280 & 2.869417 & 2.092992 \\
\hline 1 & -5.565257 & 1.572816 & 3.276901 \\
\hline 1 & -4.511570 & 2.941187 & 3.658069 \\
\hline 6 & -3.034065 & -1.297994 & -3.261827 \\
\hline 1 & -3.928680 & -1.881414 & -3.505085 \\
\hline 1 & -2.484167 & -1.146764 & -4.195320 \\
\hline 1 & -2.407649 & -1.885317 & -2.588659 \\
\hline 6 & -4.393662 & 0.783732 & -3.562952 \\
\hline 1 & -3.995344 & 0.862885 & -4.579295 \\
\hline 1 & -5.335996 & 0.226758 & -3.609535 \\
\hline 1 & -4.617407 & 1.791895 & -3.206562 \\
\hline 6 & -7.825992 & -1.724923 & 0.419064 \\
\hline 1 & -7.984664 & -0.939792 & 1.165824 \\
\hline 1 & -7.945081 & -1.276121 & -0.570530 \\
\hline 1 & -8.600734 & -2.486551 & 0.552110 \\
\hline 6 & -6.300177 & -3.013930 & 1.947532 \\
\hline 1 & -6.990959 & -3.859314 & 2.015872 \\
\hline 1 & -5.284676 & -3.383800 & 2.121338 \\
\hline 1 & -6.547586 & -2.324178 & 2.760566 \\
\hline 6 & 3.505750 & -0.177542 & 2.745241 \\
\hline 1 & 2.677398 & 0.532011 & 2.661442 \\
\hline 6 & 3.792428 & 0.874071 & -2.278012 \\
\hline 1 & 3.170962 & 1.719270 & -1.970715 \\
\hline 6 & 6.402560 & -2.953361 & -0.305053 \\
\hline 1 & 6.635014 & -3.348418 & 0.69200 \\
\hline 6 & 5.782178 & -4.087618 & -1.127733 \\
\hline 1 & 6.502506 & -4.898090 & -1.275553 \\
\hline 1 & 4.901062 & -4.502089 & -0.628458 \\
\hline 1 & 5.474169 & -3.725970 & -2.115071 \\
\hline 6 & 7.713640 & -2.479557 & -0.94188 \\
\hline 1 & 7.542167 & -2.110203 & -1.958153 \\
\hline 1 & 8.162261 & -1.668862 & -0.362169 \\
\hline 1 & 8.432472 & -3.302175 & -1.002830 \\
\hline 6 & 2.959784 & 0.008136 & -3.231708 \\
\hline 1 & 3.540217 & -0.851290 & -3.58528 \\
\hline 1 & 2.056821 & -0.358877 & -2.735680 \\
\hline 1 & 2.656552 & 0.589466 & -4.107830 \\
\hline 6 & 5.025016 & 1.449997 & -2.984829 \\
\hline 1 & 5.608184 & 2.079452 & -2.306853 \\
\hline 1 & 5.681124 & 0.654400 & -3.352425 \\
\hline 1 & 4.723239 & 2.056058 & -3.84427 \\
\hline 6 & 4.560036 & 0.404800 & 3.694565 \\
\hline 1 & 4.139048 & 0.544400 & 4.694920 \\
\hline
\end{tabular}




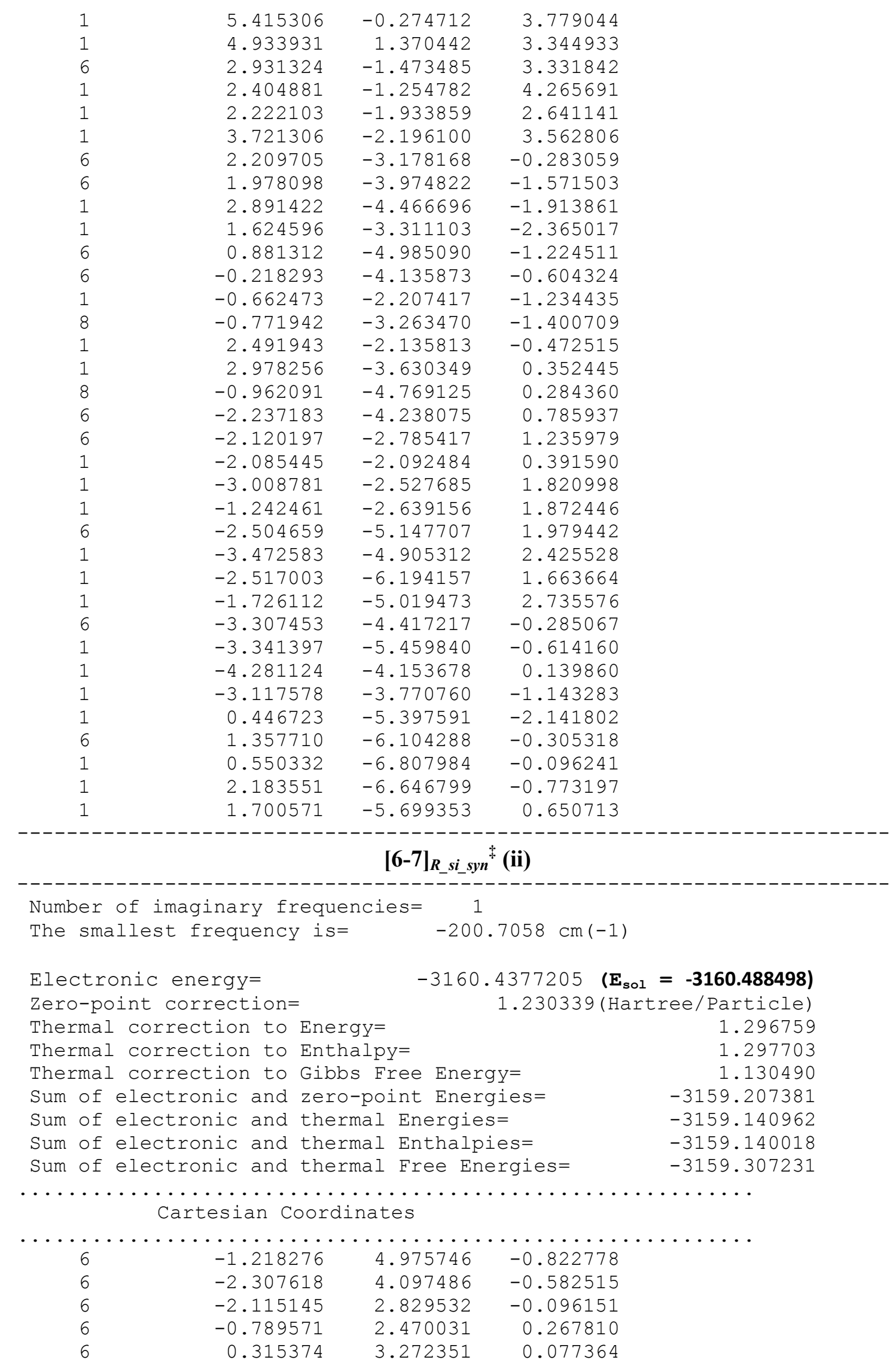




\begin{tabular}{|c|c|c|c|}
\hline 6 & 0.112549 & 4.550975 & -0.543531 \\
\hline 1 & -3.310064 & 4.421235 & -0.850505 \\
\hline 6 & 3.881581 & 2.937644 & 1.496515 \\
\hline 6 & 4.217588 & 1.663099 & 0.970755 \\
\hline 6 & 3.304221 & 0.905739 & 0.282579 \\
\hline 6 & 2.040234 & 1.494701 & 0.013924 \\
\hline 6 & 1.661430 & 2.750038 & 0.447327 \\
\hline 6 & 2.581588 & 3.475127 & 1.276244 \\
\hline 1 & 5.214980 & 1.269525 & 1.145913 \\
\hline 6 & 3.156182 & 5.366172 & 2.688767 \\
\hline 6 & 2.240851 & 4.696620 & 1.916901 \\
\hline 1 & 2.872534 & 6.293839 & 3.175299 \\
\hline 1 & 1.238790 & 5.092853 & 1.796480 \\
\hline 6 & 4.463424 & 4.855637 & 2.864111 \\
\hline 6 & 4.812140 & 3.664791 & 2.283936 \\
\hline 1 & 5.179365 & 5.400337 & 3.470798 \\
\hline 1 & 5.803418 & 3.244418 & 2.430466 \\
\hline 6 & 0.943091 & 6.621869 & -1.506427 \\
\hline 6 & 1.184229 & 5.401321 & -0.928499 \\
\hline 1 & 1.777639 & 7.251839 & -1.796845 \\
\hline 1 & 2.204427 & 5.070677 & -0.769436 \\
\hline 6 & -0.380372 & 7.062973 & -1.736281 \\
\hline 6 & -1.433918 & 6.251589 & -1.407209 \\
\hline 1 & -0.555750 & 8.033551 & -2.188635 \\
\hline 1 & -2.456369 & 6.562511 & -1.603768 \\
\hline 8 & 1.173728 & 0.753917 & -0.769739 \\
\hline 8 & -0.627670 & 1.241438 & 0.877479 \\
\hline 15 & -0.050856 & 0.016282 & -0.004377 \\
\hline 8 & 0.423094 & -0.999889 & 1.007878 \\
\hline 8 & -0.980083 & -0.458137 & -1.089951 \\
\hline 1 & 0.186323 & -2.305768 & 0.623494 \\
\hline 6 & 3.601181 & -0.492239 & -0.162658 \\
\hline 6 & 3.671510 & -1.527878 & 0.790459 \\
\hline 6 & 3.803888 & -0.777427 & -1.527024 \\
\hline 6 & 4.024373 & -2.812755 & 0.366431 \\
\hline 6 & 4.141794 & -2.077911 & -1.901100 \\
\hline 6 & 4.275981 & -3.107524 & -0.970520 \\
\hline 6 & -3.187480 & 1.788780 & -0.057289 \\
\hline 6 & -3.695184 & 1.289041 & -1.277197 \\
\hline 6 & -3.621025 & 1.239179 & 1.162473 \\
\hline 6 & -4.644262 & 0.268871 & -1.240742 \\
\hline 6 & -4.566570 & 0.212283 & 1.143460 \\
\hline 6 & -5.083317 & -0.294664 & -0.044894 \\
\hline 8 & 0.055457 & -3.271124 & 0.186524 \\
\hline 1 & -4.906529 & -0.214143 & 2.085980 \\
\hline 1 & -5.036791 & -0.112941 & -2.180260 \\
\hline 1 & 4.305430 & -2.299899 & -2.955064 \\
\hline 1 & 4.082824 & -3.604122 & 1.107196 \\
\hline 6 & -3.118650 & 1.735679 & 2.509713 \\
\hline 1 & -2.338930 & 2.483061 & 2.334423 \\
\hline 6 & -3.239512 & 1.797979 & -2.639092 \\
\hline 1 & -2.341454 & 2.404885 & -2.497352 \\
\hline 6 & -6.097858 & -1.421251 & -0.038827 \\
\hline 1 & -6.148106 & -1.807439 & 0.988135 \\
\hline 6 & -2.498726 & 0.604325 & 3.338426 \\
\hline 1 & -1.664325 & 0.141232 & 2.805612 \\
\hline 1 & -2.124316 & 0.994750 & 4.290011 \\
\hline 7 & -3.239439 & -0.170102 & 3.567222 \\
\hline
\end{tabular}




\begin{tabular}{|c|c|c|c|}
\hline 6 & -4.250890 & 2.423967 & 3.282718 \\
\hline 1 & -4.674455 & 3.251324 & 2.706300 \\
\hline 1 & -5.059075 & 1.717999 & 3.500774 \\
\hline 1 & -3.882022 & 2.818340 & 4.234544 \\
\hline 6 & -2.860058 & 0.658142 & -3.591618 \\
\hline 1 & -3.731788 & 0.058727 & -3.874644 \\
\hline 1 & -2.438013 & 1.071845 & -4.512843 \\
\hline 1 & -2.118376 & 0.005771 & -3.126252 \\
\hline 6 & -4.319806 & 2.684609 & -3.271609 \\
\hline 1 & -3.982229 & 3.075559 & -4.236569 \\
\hline 1 & -5.235662 & 2.107707 & -3.440959 \\
\hline 1 & -4.578317 & 3.531238 & -2.629050 \\
\hline 6 & -5.672962 & -2.573192 & -0.955977 \\
\hline 1 & -5.710748 & -2.268846 & -2.007317 \\
\hline 1 & -4.649947 & -2.889474 & -0.733296 \\
\hline 1 & -6.345835 & -3.428440 & -0.836166 \\
\hline 6 & -7.489845 & -0.905291 & -0.421133 \\
\hline 1 & -8.227599 & -1.713388 & -0.391246 \\
\hline 1 & -7.815785 & -0.111879 & 0.256755 \\
\hline 1 & -7.476974 & -0.494969 & -1.436553 \\
\hline 6 & 3.353701 & -1.303037 & 2.262819 \\
\hline 1 & 2.739214 & -0.400504 & 2.333870 \\
\hline 6 & 3.663098 & 0.278556 & -2.611705 \\
\hline 1 & 3.377417 & 1.223519 & -2.141053 \\
\hline 6 & 4.702953 & -4.486463 & -1.443701 \\
\hline 1 & 4.185684 & -4.671982 & -2.395439 \\
\hline 6 & 6.212368 & -4.497158 & -1.722938 \\
\hline 1 & 6.532034 & -5.469415 & -2.110595 \\
\hline 1 & 6.485536 & -3.727370 & -2.449610 \\
\hline 1 & 6.764327 & -4.297148 & -0.798680 \\
\hline 6 & 4.335793 & -5.614931 & -0.480563 \\
\hline 1 & 4.921616 & -5.551741 & 0.442343 \\
\hline 1 & 3.276200 & -5.590798 & -0.207664 \\
\hline 1 & 4.550680 & -6.584810 & -0.937509 \\
\hline 6 & 2.555829 & -0.097764 & -3.603266 \\
\hline 1 & 2.807094 & -1.017850 & -4.142273 \\
\hline 1 & 1.604380 & -0.245654 & -3.085009 \\
\hline 1 & 2.422820 & 0.695544 & -4.345214 \\
\hline 6 & 4.996664 & 0.511133 & -3.331506 \\
\hline 1 & 5.778148 & 0.804269 & -2.624718 \\
\hline 1 & 5.330690 & -0.396552 & -3.844962 \\
\hline 1 & 4.894531 & 1.301604 & -4.081114 \\
\hline 6 & 4.630625 & -1.100347 & 3.088454 \\
\hline 1 & 4.383523 & -0.970310 & 4.146836 \\
\hline 1 & 5.284111 & -1.975180 & 2.998091 \\
\hline 1 & 5.196034 & -0.224150 & 2.763495 \\
\hline 6 & 2.537338 & -2.455536 & 2.861543 \\
\hline 1 & 2.109993 & -2.146573 & 3.82002 \\
\hline 1 & 1.720638 & -2.746660 & 2.198688 \\
\hline 1 & 3.161987 & -3.335492 & 3.051151 \\
\hline 6 & 0.790198 & -3.273580 & -1.04755 \\
\hline 6 & -0.253239 & -3.334636 & -2.165045 \\
\hline 7 & 0.143931 & -3.822516 & -3.05861 \\
\hline 1 & -0.568070 & -2.324020 & -2.43479 \\
\hline 6 & -1.420852 & -4.129057 & -1.572113 \\
\hline 6 & -1.669684 & -3.539865 & -0.183989 \\
\hline 1 & -1.778717 & -1.590056 & -0.61870 \\
\hline 8 & -2.221427 & -2.354109 & -0.07228 \\
\hline
\end{tabular}




\begin{tabular}{|c|c|c|c|}
\hline 1 & 1.415935 & -2.374745 & -1.093886 \\
\hline 1 & 1.446492 & -4.148416 & -1.046609 \\
\hline 8 & -2.068420 & -4.441494 & 0.697590 \\
\hline 6 & -2.476339 & -4.097246 & 2.065725 \\
\hline 6 & -1.417601 & -3.239344 & 2.746602 \\
\hline 1 & -1.321796 & -2.258778 & 2.276485 \\
\hline 1 & -1.709667 & -3.086946 & 3.789893 \\
\hline 1 & -0.446657 & -3.741526 & 2.727011 \\
\hline 6 & -2.563789 & -5.469258 & 2.724096 \\
\hline 1 & -2.865283 & -5.360391 & 3.768909 \\
\hline 1 & -3.300367 & -6.092234 & 2.209659 \\
\hline 1 & -1.593551 & -5.970625 & 2.686890 \\
\hline 6 & -3.844173 & -3.420962 & 2.058181 \\
\hline 1 & -4.551713 & -4.007737 & 1.463889 \\
\hline 1 & -4.218575 & -3.370285 & 3.085616 \\
\hline 1 & -3.786278 & -2.410669 & 1.651396 \\
\hline 1 & -2.339140 & -3.941647 & -2.139521 \\
\hline 6 & -1.135389 & -5.628027 & -1.511174 \\
\hline 1 & -1.991178 & -6.176280 & -1.115577 \\
\hline 1 & -0.910253 & -6.001543 & -2.513249 \\
\hline 1 & -0.281179 & -5.837013 & -0.860687 \\
\hline
\end{tabular}

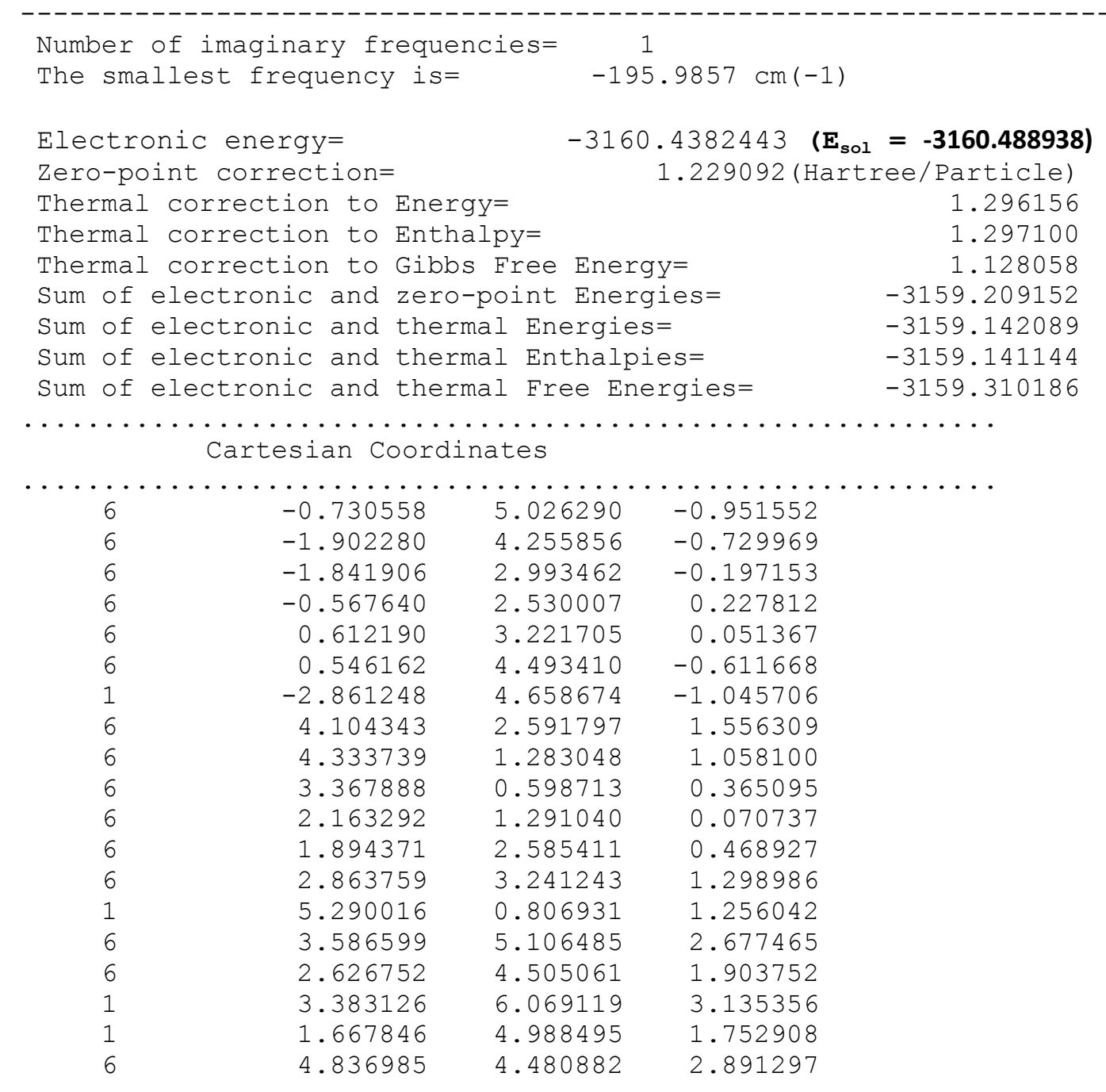




\begin{tabular}{|c|c|c|c|}
\hline 6 & 5.083787 & 3.248883 & 2.345971 \\
\hline 1 & 5.590080 & 4.971769 & 3.498928 \\
\hline 1 & 6.029863 & 2.744137 & 2.521300 \\
\hline 6 & 1.592336 & 6.454971 & -1.594175 \\
\hline 6 & 1.702290 & 5.233950 & -0.978592 \\
\hline 1 & 2.489272 & 7.000833 & -1.868335 \\
\hline 1 & 2.682690 & 4.818880 & -0.774513 \\
\hline 6 & 0.322516 & 7.005161 & -1.883532 \\
\hline 6 & -0.810221 & 6.299145 & -1.575503 \\
\hline 1 & 0.249294 & 7.975164 & -2.364353 \\
\hline 1 & -1.793144 & 6.694365 & -1.817172 \\
\hline 8 & 1.243779 & 0.610947 & -0.706580 \\
\hline 8 & -0.540084 & 1.314048 & 0.882265 \\
\hline 15 & -0.052605 & 0.010116 & 0.060911 \\
\hline 8 & 0.311174 & -1.001183 & 1.122318 \\
\hline 8 & -0.996191 & -0.425758 & -1.029318 \\
\hline 1 & -0.039131 & -2.297895 & 0.784038 \\
\hline 6 & 3.545451 & -0.823509 & -0.065538 \\
\hline 6 & 3.538421 & -1.852442 & 0.900278 \\
\hline 6 & 3.710155 & -1.138660 & -1.427021 \\
\hline 6 & 3.769675 & -3.163550 & 0.481572 \\
\hline 6 & 3.932001 & -2.467891 & -1.795599 \\
\hline 6 & 3.977381 & -3.494081 & -0.856170 \\
\hline 6 & -3.003829 & 2.053243 & -0.159377 \\
\hline 6 & -3.508157 & 1.541447 & -1.375453 \\
\hline 6 & -3.527297 & 1.600678 & 1.064922 \\
\hline 6 & -4.543523 & 0.609194 & -1.332359 \\
\hline 6 & -4.558453 & 0.659931 & 1.053281 \\
\hline 6 & -5.074415 & 0.143715 & -0.131461 \\
\hline 8 & -0.243846 & -3.263512 & 0.382823 \\
\hline 1 & -4.969578 & 0.311403 & 1.999347 \\
\hline 1 & -4.934378 & 0.219164 & -2.269055 \\
\hline 1 & 4.069251 & -2.698749 & -2.849170 \\
\hline 1 & 3.769737 & -3.962671 & 1.220201 \\
\hline 6 & -3.027946 & 2.113821 & 2.406555 \\
\hline 1 & -2.163892 & 2.760933 & 2.228370 \\
\hline 6 & -2.960996 & 1.949965 & -2.736938 \\
\hline 1 & -2.013906 & 2.473929 & -2.582818 \\
\hline 6 & -6.186167 & -0.887267 & -0.115646 \\
\hline 1 & -6.297648 & -1.228000 & 0.922580 \\
\hline 6 & -2.568206 & 0.971367 & 3.318735 \\
\hline 1 & -1.778828 & 0.386962 & 2.839391 \\
\hline 1 & -2.176513 & 1.372410 & 4.258749 \\
\hline 1 & -3.398536 & 0.300282 & 3.566297 \\
\hline 6 & -4.106520 & 2.965149 & 3.088391 \\
\hline 1 & -4.407871 & 3.801395 & 2.451095 \\
\hline 1 & -4.998806 & 2.367249 & 3.302204 \\
\hline 1 & -3.736015 & 3.368736 & 4.035806 \\
\hline 6 & -2.660115 & 0.744671 & -3.635793 \\
\hline 1 & -3.574222 & 0.216489 & -3.926510 \\
\hline 1 & -2.175115 & 1.084117 & -4.556493 \\
\hline 1 & -1.995117 & 0.045978 & -3.124084 \\
\hline 6 & -3.931367 & 2.908440 & -3.439113 \\
\hline 1 & -3.527389 & 3.230886 & -4.403856 \\
\hline 1 & -4.890587 & 2.411573 & -3.621887 \\
\hline 1 & -4.131821 & 3.798195 & -2.835254 \\
\hline 6 & -5.844550 & -2.107307 & -0.977953 \\
\hline 1 & -5.827026 & -1.842044 & -2.040391 \\
\hline
\end{tabular}




\begin{tabular}{|c|c|c|c|}
\hline 1 & -4.861243 & -2.506626 & -0.713262 \\
\hline 1 & -6.596555 & -2.892021 & -0.846983 \\
\hline 6 & -7.515545 & -0.263279 & -0.555719 \\
\hline 1 & -8.324703 & -0.999342 & -0.515116 \\
\hline & -7.783694 & 0.583486 & 0.081680 \\
\hline & -7.440912 & 0.102579 & -1.585357 \\
\hline 6 & 3.266550 & -1.586315 & 2.374602 \\
\hline 1 & 2.765328 & -0.616906 & 2.451808 \\
\hline 6 & 3.651320 & -0.087007 & -2.523659 \\
\hline 1 & 3.443595 & 0.883253 & -2.063855 \\
\hline 6 & 4.266146 & -4.932059 & -1.245458 \\
\hline 1 & 3.835270 & -5.569089 & -0.461469 \\
\hline 6 & 3.637240 & -5.338833 & -2.579870 \\
\hline 1 & 3.761614 & -6.412752 & -2.745591 \\
\hline 1 & 2.567762 & -5.107508 & -2.609035 \\
\hline 1 & 4.115948 & -4.822820 & -3.418088 \\
\hline 6 & 5.781024 & -5.175299 & -1.270422 \\
\hline 1 & 6.251037 & -4.537488 & -2.026560 \\
\hline 1 & 6.232258 & -4.937542 & -0.303479 \\
\hline 1 & 6.006717 & -6.218409 & -1.513062 \\
\hline 6 & 2.514045 & -0.385100 & -3.50854 \\
\hline 1 & 2.693828 & -1.323770 & -4.044278 \\
\hline 1 & 1.556824 & -0.460216 & -2.985182 \\
\hline 1 & 2.436717 & 0.412660 & -4.253577 \\
\hline 6 & 4.996527 & 0.029862 & -3.249777 \\
\hline 1 & 5.802068 & 0.265570 & -2.548599 \\
\hline 1 & 5.254203 & -0.906022 & -3.756489 \\
\hline 1 & 4.954889 & 0.819342 & -4.006316 \\
\hline 6 & 4.573390 & -1.533960 & 3.177089 \\
\hline 1 & 4.365216 & -1.358437 & 4.237105 \\
\hline 1 & 5.108465 & -2.485882 & 3.088500 \\
\hline 1 & 5.238956 & -0.742084 & 2.82596 \\
\hline 6 & 2.329772 & -2.632916 & 2.990445 \\
\hline 1 & 1.977868 & -2.285057 & 3.966075 \\
\hline 1 & 1.460426 & -2.806600 & 2.35387 \\
\hline 1 & 2.842204 & -3.587820 & 3.149653 \\
\hline 6 & 0.524402 & -3.385947 & -0.823406 \\
\hline 6 & -0.486959 & -3.406784 & -1.971908 \\
\hline 1 & -0.108255 & -3.963443 & -2.833135 \\
\hline 1 & -0.706005 & -2.384901 & -2.29076 \\
\hline 6 & -1.736489 & -4.072187 & -1.387723 \\
\hline 6 & -1.982434 & -3.398523 & -0.038513 \\
\hline 1 & -1.897383 & -1.464711 & -0.55369 \\
\hline 8 & -2.428888 & -2.166700 & 0.00158 \\
\hline 1 & 1.226655 & -2.546484 & -0.89121 \\
\hline 1 & 1.102404 & -4.312925 & -0.759660 \\
\hline 8 & -2.483246 & -4.218690 & 0.868259 \\
\hline 6 & -2.896160 & -3.773488 & 2.205932 \\
\hline 6 & -1.789149 & -2.968695 & 2.87533 \\
\hline 1 & -1.607659 & -2.021685 & 2.36365 \\
\hline 1 & -2.092998 & -2.745846 & 3.90243 \\
\hline 1 & -0.859154 & -3.542838 & 2.904457 \\
\hline 6 & -3.111172 & -5.099257 & 2.92700 \\
\hline 1 & -3.422800 & -4.914148 & 3.95799 \\
\hline 1 & -3.887180 & -5.683872 & 2.42574 \\
\hline 1 & -2.185827 & -5.680239 & 2.93714 \\
\hline 6 & -4.204217 & -2.990987 & 2.12527 \\
\hline 1 & -4.939475 & -3.545450 & 1.53354 \\
\hline
\end{tabular}




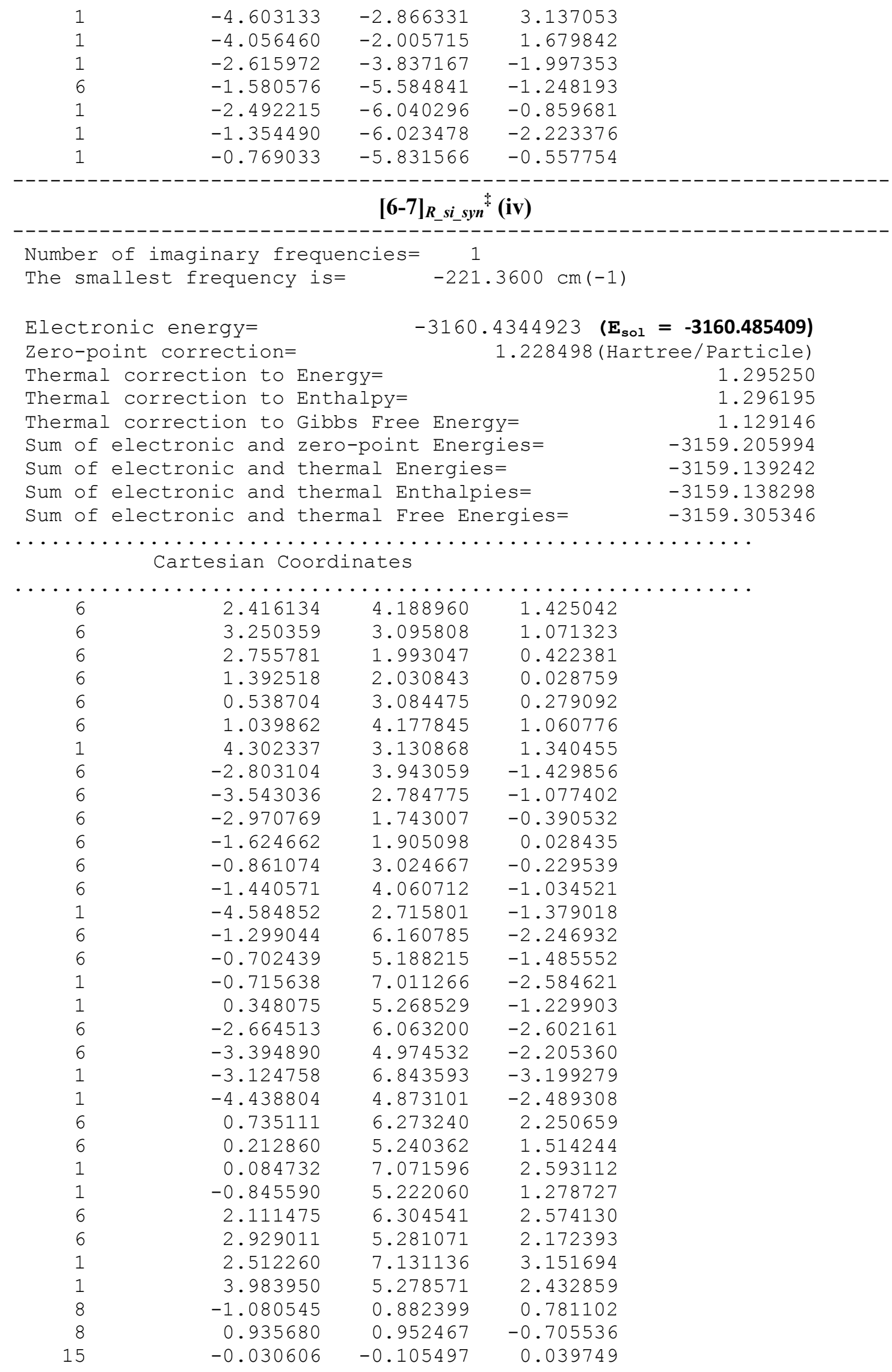




\begin{tabular}{|c|c|c|c|}
\hline 8 & -0.668831 & -0.911571 & -1.059550 \\
\hline 8 & 0.656929 & -0.836275 & 1.165732 \\
\hline 1 & -0.783518 & -2.248517 & -0.671569 \\
\hline 6 & -3.736125 & 0.490208 & -0.097236 \\
\hline 6 & -4.100265 & -0.367651 & -1.158647 \\
\hline 6 & -4.140955 & 0.190476 & 1.215902 \\
\hline 6 & -4.959226 & -1.434531 & -0.895527 \\
\hline 6 & -4.977644 & -0.909395 & 1.431442 \\
\hline 6 & -5.427289 & -1.712931 & 0.388721 \\
\hline 6 & 3.598880 & 0.794310 & 0.116366 \\
\hline 6 & 3.973061 & -0.084941 & 1.153257 \\
\hline 6 & 4.024117 & 0.551582 & -1.202031 \\
\hline 6 & 4.831937 & -1.143675 & 0.852455 \\
\hline 6 & 4.884369 & -0.519665 & -1.451539 \\
\hline 6 & 5.315339 & -1.368360 & -0.435484 \\
\hline 8 & -1.018607 & -3.195693 & -0.274384 \\
\hline 1 & 5.225579 & -0.686730 & -2.470630 \\
\hline 1 & 5.139834 & -1.820634 & 1.646910 \\
\hline 1 & -5.307330 & -1.132114 & 2.444583 \\
\hline 1 & -5.264261 & -2.074186 & -1.721253 \\
\hline 6 & 3.585997 & 1.416470 & -2.373563 \\
\hline 1 & 2.914091 & 2.194379 & -1.999883 \\
\hline 6 & 3.476932 & 0.088652 & 2.581851 \\
\hline 1 & 2.560991 & 0.686998 & 2.545066 \\
\hline 6 & 6.331607 & -2.467879 & -0.683874 \\
\hline 1 & 6.203209 & -3.213052 & 0.112514 \\
\hline 6 & 2.805779 & 0.588203 & -3.401286 \\
\hline 1 & 1.946182 & 0.100741 & -2.933859 \\
\hline 1 & 2.442415 & 1.229663 & -4.210218 \\
\hline 1 & 3.442997 & -0.182861 & -3.8 \\
\hline 6 & 4.783344 & 2.120809 & -3.021988 \\
\hline 1 & 5.324537 & 2.728536 & -2.291138 \\
\hline 1 & 5.486891 & 1.395051 & -3.442895 \\
\hline 1 & 4.450151 & 2.773448 & -3.834685 \\
\hline 6 & 3.120735 & -1.245486 & 3.245536 \\
\hline 1 & 4.017227 & -1.826044 & 3.488858 \\
\hline 1 & 2.592082 & -1.059928 & 4.185052 \\
\hline 1 & 2.477519 & -1.851070 & 2.605220 \\
\hline 6 & 4.513990 & 0.825290 & 3.440565 \\
\hline 1 & 4.151424 & 0.934592 & 4.467484 \\
\hline 1 & 5.451193 & 0.258532 & 3.469602 \\
\hline 1 & 4.736568 & 1.821503 & 3.051421 \\
\hline 6 & 7.752413 & -1.899185 & -0.565363 \\
\hline 1 & 7.914830 & -1.132925 & -1.330699 \\
\hline 1 & 7.908136 & -1.435415 & 0.412232 \\
\hline 1 & 8.502055 & -2.684911 & -0.701895 \\
\hline 6 & 6.152803 & -3.174219 & -2.029191 \\
\hline 1 & 6.817990 & -4.040098 & -2.095494 \\
\hline 1 & 5.123506 & -3.519158 & -2.170623 \\
\hline 1 & 6.399614 & -2.509205 & -2.862748 \\
\hline 6 & -3.567919 & -0.186617 & -2.573668 \\
\hline 1 & -2.721721 & 0.505184 & -2.526177 \\
\hline 6 & -3.706000 & 1.012767 & 2.41914 \\
\hline 1 & -3.081901 & 1.839487 & 2.06941 \\
\hline 6 & -6.402369 & -2.849055 & 0.63846 \\
\hline 1 & -6.629532 & -2.852994 & 1.71202 \\
\hline 6 & -7.714454 & -2.619093 & -0.12007 \\
\hline 1 & -8.440513 & -3.403181 & 0.11519 \\
\hline
\end{tabular}




\begin{tabular}{|c|c|c|c|}
\hline 1 & -8.152293 & -1.651332 & 0.137588 \\
\hline 1 & -7.544209 & -2.633422 & -1.201515 \\
\hline 6 & -5.805767 & -4.214069 & 0.278792 \\
\hline 1 & -5.519713 & -4.249316 & -0.778124 \\
\hline 1 & -4.915026 & -4.430800 & 0.876114 \\
\hline 1 & -6.533999 & -5.011836 & 0.454238 \\
\hline 6 & -2.857194 & 0.163052 & 3.373370 \\
\hline 1 & -3.437324 & -0.679140 & 3.766509 \\
\hline 1 & -1.972652 & -0.228529 & 2.862857 \\
\hline 1 & -2.523067 & 0.764551 & 4.224316 \\
\hline 6 & -4.910224 & 1.625384 & 3.143491 \\
\hline 1 & -5.504953 & 2.242415 & 2.464133 \\
\hline 1 & -5.564528 & 0.849273 & 3.553056 \\
\hline 1 & -4.576163 & 2.252467 & 3.975479 \\
\hline 6 & -4.634356 & 0.401376 & -3.505656 \\
\hline 1 & -4.238528 & 0.509558 & -4.520077 \\
\hline 1 & -5.507910 & -0.258407 & -3.551261 \\
\hline 1 & -4.974614 & 1.383157 & -3.167094 \\
\hline 6 & -3.038546 & -1.505957 & -3.150184 \\
\hline 1 & -2.523264 & -1.315055 & -4.096190 \\
\hline 1 & -2.329601 & -1.975328 & -2.465288 \\
\hline 1 & -3.850629 & -2.211021 & -3.357255 \\
\hline 6 & -2.229996 & -3.067318 & 0.481441 \\
\hline 6 & -1.974333 & -3.811296 & 1.796024 \\
\hline 1 & -2.891424 & -4.247475 & 2.200081 \\
\hline 1 & -1.560050 & -3.127393 & 2.541448 \\
\hline 6 & -0.934641 & -4.881300 & 1.450184 \\
\hline 6 & 0.170746 & -4.105055 & 0.749599 \\
\hline 1 & 0.689082 & -2.166353 & 1.293569 \\
\hline 8 & 0.792996 & -3.221383 & 1.479459 \\
\hline 1 & -2.465957 & -2.007187 & 0.631022 \\
\hline 1 & -3.039101 & -3.521313 & -0.098305 \\
\hline 8 & 0.854117 & -4.805388 & -0.137383 \\
\hline 6 & 2.120487 & -4.332566 & -0.714389 \\
\hline 6 & 2.021374 & -2.895348 & -1.217651 \\
\hline 1 & 2.058988 & -2.167330 & -0.403253 \\
\hline 1 & 2.877460 & -2.690052 & -1.868140 \\
\hline 1 & 1.109366 & -2.748338 & -1.803809 \\
\hline 6 & 2.310692 & -5.294320 & -1.881750 \\
\hline 1 & 3.266774 & -5.098698 & -2.374328 \\
\hline 1 & 2.304908 & -6.328235 & -1.526992 \\
\hline 1 & 1.506643 & -5.170112 & -2.611440 \\
\hline 6 & 3.231038 & -4.503374 & 0.315895 \\
\hline 1 & 3.238493 & -5.529336 & 0.695132 \\
\hline 1 & 4.194261 & -4.304453 & -0.163887 \\
\hline 1 & 3.105418 & -3.809119 & 1.147695 \\
\hline 1 & -0.478343 & -5.272831 & 2.366101 \\
\hline 6 & -1.495102 & -6.016799 & 0.601069 \\
\hline 1 & -0.725093 & -6.759507 & 0.385667 \\
\hline 1 & -2.318105 & -6.508093 & 1.127038 \\
\hline 1 & -1.867301 & -5.637426 & -0.354468 \\
\hline
\end{tabular}

$[6-7]_{S_{-} r e-a n t i}{ }^{*}(\mathrm{i})$

$\begin{array}{ll}\text { Number of imaginary frequencies }= & 1 \\ \text { The smallest frequency is }= & -199.4496 \mathrm{~cm}(-1) \\ \text { Electronic energy }= & -3160.4409325 \quad\left(\mathbf{E}_{\text {sol }}=\mathbf{- 3 1 6 0 . 4 9 2 1 2}\right) \\ & \text { S106 }\end{array}$




$\begin{array}{lr}\text { Zero-point correction= } & \text { 1.230424 (Hartree/Particle) } \\ \text { Thermal correction to Energy= } & 1.297013 \\ \text { Thermal correction to Enthalpy= } & 1.297957 \\ \text { Thermal correction to Gibbs Free Energy= } & 1.131000 \\ \text { Sum of electronic and zero-point Energies= } & -3159.210509 \\ \text { Sum of electronic and thermal Energies= } & -3159.143919 \\ \text { Sum of electronic and thermal Enthalpies= } & -3159.142975 \\ \text { Sum of electronic and thermal Free Energies= } & -3159.309933\end{array}$

Cartesian Coordinates

\begin{tabular}{|c|c|c|c|}
\hline 6 & 3.576056 & 2.845332 & 1.186459 \\
\hline 6 & 3.020862 & 1.803916 & 0.487590 \\
\hline 6 & 1.695476 & 1.978248 & 0.006438 \\
\hline 6 & 0.930562 & 3.103759 & 0.227654 \\
\hline 6 & 1.489581 & 4.143991 & 1.041993 \\
\hline 1 & 4.603647 & 2.771232 & 1.531642 \\
\hline 6 & -2.302801 & 4.212180 & -1.555323 \\
\hline 6 & -3.138224 & 3.118233 & -1.206230 \\
\hline 6 & -2.656766 & 2.041030 & -0.506613 \\
\hline 6 & -1.307380 & 2.100126 & -0.069191 \\
\hline 6 & -0.455104 & 3.155189 & -0.319514 \\
\hline 6 & -0.940959 & 4.226527 & -1.139275 \\
\hline 1 & -4.178354 & 3.132474 & -1.520444 \\
\hline 6 & -0.615209 & 6.298244 & -2.364143 \\
\hline 6 & -0.109369 & 5.288585 & -1.585291 \\
\hline 1 & 0.038233 & 7.096861 & -2.700070 \\
\hline 1 & 0.939773 & 5.287172 & -1.310387 \\
\hline 6 & -1.978481 & 6.304663 & -2.740676 \\
\hline 6 & -2.798992 & 5.280414 & -2.347022 \\
\hline 1 & -2.366135 & 7.112712 & -3.352355 \\
\hline 1 & -3.843139 & 5.259419 & -2.647182 \\
\hline 6 & 1.323203 & 6.262648 & 2.219248 \\
\hline 6 & 0.746745 & 5.285180 & 1.448292 \\
\hline 1 & 0.736602 & 7.124329 & 2.521111 \\
\hline 1 & -0.291804 & 5.373263 & 1.149423 \\
\hline 6 & 2.671614 & 6.154688 & 2.630698 \\
\hline 6 & 3.404541 & 5.052021 & 2.278677 \\
\hline 1 & 3.116775 & 6.937956 & 3.235475 \\
\hline 1 & 4.434772 & 4.943487 & 2.606460 \\
\hline 8 & -0.854796 & 1.037767 & 0.689913 \\
\hline 8 & 1.176011 & 0.956543 & -0.768965 \\
\hline 15 & 0.105172 & -0.031837 & -0.067583 \\
\hline 8 & -0.623820 & -0.753685 & -1.167911 \\
\hline 8 & 0.735271 & -0.856707 & 1.027775 \\
\hline 1 & 0.186757 & -2.086231 & 1.195401 \\
\hline 8 & -0.356055 & -3.006073 & 1.317686 \\
\hline 6 & -1.625223 & -2.737706 & 1.928920 \\
\hline 6 & -2.602899 & -3.652997 & 1.205603 \\
\hline 6 & -2.162675 & -3.542227 & -0.248161 \\
\hline 6 & -0.657904 & -3.770385 & -0.241821 \\
\hline 8 & 0.093879 & -3.135046 & -1.106466 \\
\hline 1 & -1.549653 & -2.927434 & 3.002393 \\
\hline 1 & -1.896846 & -1.686122 & 1.760956 \\
\hline 6 & 2.833885 & 4.016529 & 1.493462 \\
\hline 8 & -0.335908 & -5.036972 & -0.005111 \\
\hline 6 & 1.049240 & -5.511389 & -0.087031 \\
\hline 6 & 1.438973 & -5.677475 & -1.553585 \\
\hline
\end{tabular}




\begin{tabular}{|c|c|c|c|}
\hline L & 2.380529 & -6.231987 & -1.615068 \\
\hline 1 & 0.667760 & -6.247397 & -2.079754 \\
\hline[ & 1.562704 & -4.712006 & -2.043953 \\
\hline 6 & 0.966718 & -6.870390 & 0.598530 \\
\hline & 1.949777 & -7.347951 & 0.598301 \\
\hline & 0.631681 & -6.753110 & 1.632120 \\
\hline 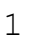 & 0.261425 & -7.519752 & 0.072981 \\
\hline 6 & 2.010526 & -4.593057 & 0.666164 \\
\hline 1 & 2.060667 & -3.593773 & 0.225979 \\
\hline 1 & 1.714247 & -4.502272 & 1.713856 \\
\hline 1 & 3.011524 & -5.035299 & 0.62293 \\
\hline 1 & -0.221798 & -2.156011 & -1.22994 \\
\hline 6 & 3.739511 & 0.521816 & 0.20681 \\
\hline 6 & 4.199532 & 0.258629 & -1.096794 \\
\hline 6 & 3.910992 & -0.434863 & 1.226494 \\
\hline 6 & 4.854575 & -0.944450 & -1.353025 \\
\hline 6 & 4.570611 & -1.626563 & 0.918308 \\
\hline 6 & 5.048023 & -1.902231 & -0.35974 \\
\hline 1 & 5.216182 & -1.139847 & -2.360801 \\
\hline 1 & 4.705767 & -2.375007 & 1.695377 \\
\hline 6 & -3.494797 & 0.844326 & -0.181946 \\
\hline 6 & -3.918566 & 0.628040 & 1.140117 \\
\hline 6 & -3.859507 & -0.065709 & -1.198630 \\
\hline 6 & -4.750521 & -0.461668 & 1.421666 \\
\hline 6 & -4.699385 & -1.128798 & -0.869507 \\
\hline 6 & -5.171817 & -1.339268 & 0.428016 \\
\hline 1 & -5.083801 & -0.606967 & 2.445578 \\
\hline 1 & -5.004122 & -1.823062 & -1.651165 \\
\hline 6 & -6.146194 & -2.478590 & 0.686603 \\
\hline 1 & -5.728989 & -3.377217 & 0.207912 \\
\hline 6 & -3.539231 & 1.557483 & 2.283877 \\
\hline 1 & -2.891859 & 2.346479 & 1.891328 \\
\hline 6 & 4.014703 & 1.244613 & -2.240162 \\
\hline 1 & 3.439744 & 2.099696 & -1.872431 \\
\hline 6 & 3.411125 & -0.205929 & 2.645518 \\
\hline 1 & 2.606342 & 0.534649 & 2.59881 \\
\hline 6 & 5.757276 & -3.208121 & -0.661503 \\
\hline 1 & 5.717349 & -3.817904 & 0.250758 \\
\hline 6 & -3.395246 & 0.092250 & -2.640308 \\
\hline 1 & -2.526360 & 0.756958 & -2.641448 \\
\hline 6 & -4.504036 & 0.723782 & -3.493131 \\
\hline 1 & -4.814580 & 1.696256 & -3.103194 \\
\hline 1 & -4.164966 & 0.861457 & -4.52445 \\
\hline 1 & -5.386598 & 0.074540 & -3.50906 \\
\hline 6 & -2.948141 & -1.229955 & -3.273450 \\
\hline 1 & -3.779394 & -1.935151 & -3.381647 \\
\hline 1 & -2.553660 & -1.039692 & -4.276113 \\
\hline 1 & -2.157001 & -1.696493 & -2.68398 \\
\hline 6 & -6.362432 & -2.791319 & 2.16650 \\
\hline 1 & -6.995062 & -3.676437 & 2.273945 \\
\hline 1 & -5.421407 & -2.980155 & 2.692549 \\
\hline 1 & -6.868374 & -1.961142 & 2.67039 \\
\hline 6 & -7.493524 & -2.177331 & 0.014768 \\
\hline 1 & -7.937174 & -1.278707 & 0.455513 \\
\hline 1 & -7.375497 & -2.000650 & -1.057286 \\
\hline 1 & -8.191802 & -3.008465 & 0.152613 \\
\hline 6 & 5.056944 & -3.985566 & -1.78100 \\
\hline 1 & 5.532724 & -4.959912 & -1.93209 \\
\hline
\end{tabular}




$\begin{array}{rrrr}1 & 4.000644 & -4.145051 & -1.547888 \\ 1 & 5.109948 & -3.439287 & -2.728452 \\ 6 & 7.231453 & -2.969958 & -1.007477 \\ 1 & 7.318809 & -2.365635 & -1.916640 \\ 1 & 7.744132 & -2.438964 & -0.201259 \\ 1 & 7.748046 & -3.918760 & -1.183150 \\ 6 & -2.752056 & 0.818059 & 3.372089 \\ 1 & -1.818944 & 0.416174 & 2.968868 \\ 1 & -2.504393 & 1.500306 & 4.191071 \\ 1 & -3.338220 & -0.006926 & 3.792364 \\ 6 & -4.783281 & 2.237499 & 2.868649 \\ 1 & -5.336762 & 2.775215 & 2.093731 \\ 1 & -5.460109 & 1.503553 & 3.318218 \\ 1 & -4.497459 & 2.950654 & 3.647697 \\ 6 & 4.535755 & 0.348271 & 3.531193 \\ 1 & 5.365345 & -0.365831 & 3.578491 \\ 1 & 4.930203 & 1.291953 & 3.147412 \\ 1 & 4.174789 & 0.518304 & 4.550588 \\ 6 & 2.831139 & -1.470067 & 3.289301 \\ 1 & 2.103897 & -1.946899 & 2.630213 \\ 1 & 3.615255 & -2.194445 & 3.534864 \\ 1 & 2.330714 & -1.207660 & 4.226411 \\ 6 & 5.369242 & 1.779418 & -2.720689 \\ 1 & 5.918026 & 2.250460 & -1.900055 \\ 1 & 5.990501 & 0.970699 & -3.119975 \\ 1 & 5.231329 & 2.520325 & -3.514212 \\ 6 & 3.223653 & 0.621313 & -3.395220 \\ 1 & 2.255373 & 0.254254 & -3.046675 \\ 1 & 3.052513 & 1.363758 & -4.180916 \\ 1 & 3.770241 & -0.215838 & -3.842335 \\ 1 & -2.313687 & -2.503140 & -0.571087 \\ 6 & -2.856975 & -4.487682 & -1.219858 \\ 1 & -2.756836 & -5.525019 & -0.891482 \\ 1 & -3.919832 & -4.236628 & -1.282626 \\ 1 & -2.426738 & -4.396270 & -2.221612 \\ 1 & -3.634351 & -3.323941 & 1.343530 \\ 1 & -2.502190 & -4.687046 & 1.552424\end{array}$

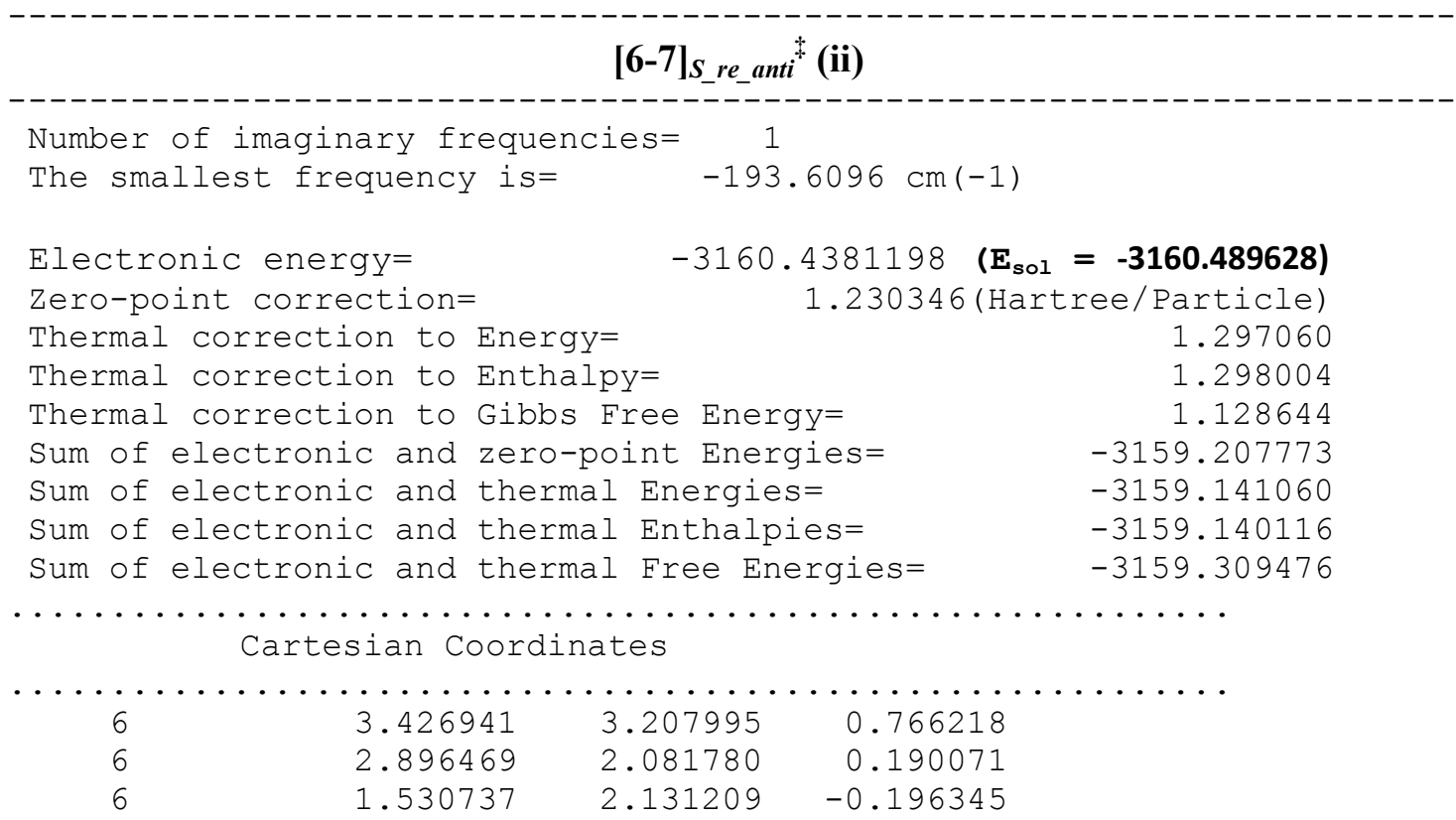




\begin{tabular}{|c|c|c|c|}
\hline 6 & 0.698124 & 3.207101 & 0.032382 \\
\hline 6 & 1.239548 & 4.334637 & 0.735826 \\
\hline 1 & 4.477374 & 3.220778 & 1.044703 \\
\hline 6 & -2.763954 & 3.979086 & -1.474446 \\
\hline 6 & -3.471537 & 2.840580 & -1.007933 \\
\hline 6 & -2.842871 & 1.834754 & -0.317982 \\
\hline 6 & -1.467842 & 2.015891 & -0.015414 \\
\hline 6 & -0.730207 & 3.121735 & -0.383921 \\
\hline 6 & -1.375355 & 4.116131 & -1.191489 \\
\hline 1 & -4.531954 & 2.755511 & -1.230022 \\
\hline 6 & -1.332613 & 6.151374 & -2.516224 \\
\hline 6 & -0.677077 & 5.218079 & -1.754155 \\
\hline 1 & -0.779721 & 6.982352 & -2.942324 \\
\hline 1 & 0.389295 & 5.309527 & -1.579824 \\
\hline 6 & -2.720946 & 6.036991 & -2.760011 \\
\hline 6 & -3.415998 & 4.970672 & -2.253664 \\
\hline 1 & -3.227812 & 6.786664 & -3.358708 \\
\hline 1 & -4.478362 & 4.856343 & -2.451114 \\
\hline 6 & 1.006370 & 6.501710 & 1.810668 \\
\hline 6 & 0.444631 & 5.435661 & 1.154961 \\
\hline 1 & 0.379601 & 7.330162 & 2.124515 \\
\hline 1 & -0.621174 & 5.423455 & 0.956740 \\
\hline 6 & 2.392669 & 6.528762 & 2.087906 \\
\hline 6 & 3.179639 & 5.467804 & 1.724887 \\
\hline 1 & 2.825560 & 7.381672 & 2.600294 \\
\hline 1 & 4.241814 & 5.460955 & 1.953757 \\
\hline 8 & -0.861963 & 1.018919 & 0.726900 \\
\hline 8 & 1.043518 & 1.034984 & -0.881825 \\
\hline 15 & 0.082981 & -0.006139 & -0.102698 \\
\hline 8 & -0.689609 & -0.751231 & -1.158851 \\
\hline 8 & 0.826268 & -0.811451 & 0.934176 \\
\hline 1 & 0.406111 & -2.110505 & 1.041465 \\
\hline 8 & -0.051009 & -3.076166 & 1.125710 \\
\hline 6 & -1.301929 & -2.960983 & 1.818960 \\
\hline 6 & -2.236714 & -3.924412 & 1.098826 \\
\hline 1 & -3.282441 & -3.692781 & 1.312710 \\
\hline 1 & -2.029375 & -4.961019 & 1.385247 \\
\hline 6 & -1.888626 & -3.702098 & -0.367219 \\
\hline 6 & -0.370543 & -3.784144 & -0.449015 \\
\hline 8 & 0.264145 & -3.037369 & -1.319590 \\
\hline 1 & -1.145619 & -3.200319 & 2.873522 \\
\hline 1 & -1.676650 & -1.931753 & 1.728694 \\
\hline 6 & 2.626230 & 4.342544 & 1.059709 \\
\hline 8 & 0.077094 & -5.026086 & -0.303874 \\
\hline 6 & 1.484337 & -5.373788 & -0.516882 \\
\hline 6 & 1.762830 & -5.449970 & -2.015972 \\
\hline 1 & 1.006509 & -6.072172 & -2.503158 \\
\hline 1 & 1.758469 & -4.460652 & -2.472366 \\
\hline 1 & 2.742648 & -5.909659 & -2.176902 \\
\hline 6 & 1.573278 & -6.759754 & 0.111575 \\
\hline 1 & 2.585463 & -7.156363 & -0.000026 \\
\hline 1 & 1.329921 & -6.709372 & 1.175792 \\
\hline 1 & 0.873062 & -7.443549 & -0.375664 \\
\hline 6 & 2.428703 & -4.405403 & 0.191747 \\
\hline 1 & 2.377020 & -3.396814 & -0.223924 \\
\hline 1 & 2.206896 & -4.359201 & 1.260830 \\
\hline 1 & 3.449895 & -4.777579 & 0.062125 \\
\hline 7 & -0.153756 & -2.089810 & -1.357897 \\
\hline
\end{tabular}




\begin{tabular}{|c|c|c|c|}
\hline 6 & 3.668024 & 0.811356 & 0.030832 \\
\hline 6 & 4.000998 & 0.329444 & -1.250486 \\
\hline 6 & 4.021373 & 0.071468 & 1.174458 \\
\hline 6 & 4.717104 & -0.859588 & -1.356012 \\
\hline 6 & 4.761800 & -1.103761 & 1.016612 \\
\hline 6 & 5.123258 & -1.587110 & -0.236031 \\
\hline 1 & 4.971057 & -1.235573 & -2.346073 \\
\hline 1 & 5.042094 & -1.659121 & 1.905746 \\
\hline 6 & -3.549459 & 0.586468 & 0.106245 \\
\hline 6 & -3.750715 & 0.324117 & 1.478175 \\
\hline 6 & -4.026644 & -0.322093 & -0.85940 \\
\hline 6 & -4.477268 & -0.806206 & 1.846643 \\
\hline 6 & -4.766101 & -1.434511 & -0.437067 \\
\hline 6 & -5.023368 & -1.682980 & 0.908189 \\
\hline 1 & -4.654292 & -0.999429 & 2.904175 \\
\hline 1 & -5.157748 & -2.112510 & -1.190660 \\
\hline 6 & -5.928779 & -2.803768 & 1.394319 \\
\hline 1 & -5.462867 & -3.231139 & 2.294005 \\
\hline 6 & -3.254794 & 1.251566 & 2.578519 \\
\hline 1 & -2.699154 & 2.073622 & 2.119998 \\
\hline 6 & 3.630276 & 1.074897 & -2.523301 \\
\hline 1 & 3.020958 & 1.941831 & -2.252244 \\
\hline 6 & 3.615930 & 0.488932 & 2.582011 \\
\hline 1 & 2.830824 & 1.246003 & 2.497097 \\
\hline 6 & 5.939440 & -2.855488 & -0.429904 \\
\hline 1 & 5.393098 & -3.477883 & -1.153751 \\
\hline 6 & -3.804408 & -0.125093 & -2.353669 \\
\hline 1 & -3.055145 & 0.660290 & -2.483615 \\
\hline 6 & -5.105335 & 0.321652 & -3.034528 \\
\hline 1 & -5.506783 & 1.235005 & -2.587079 \\
\hline 1 & -4.938005 & 0.506760 & -4.099965 \\
\hline 1 & -5.872206 & -0.455449 & -2.942597 \\
\hline 6 & -3.258189 & -1.380255 & -3.043391 \\
\hline 1 & -3.958232 & -2.220687 & -2.979507 \\
\hline 1 & -3.095084 & -1.173924 & -4.105679 \\
\hline 1 & -2.302738 & -1.675071 & -2.605932 \\
\hline 6 & -7.286594 & -2.218179 & 1.809519 \\
\hline 1 & -7.941822 & -2.998162 & 2.209404 \\
\hline 1 & -7.168465 & -1.441292 & 2.569382 \\
\hline 1 & -7.778609 & -1.766594 & 0.941873 \\
\hline 6 & -6.133530 & -3.933565 & 0.387571 \\
\hline 1 & -6.670988 & -3.580108 & -0.498515 \\
\hline 1 & -5.183572 & -4.365530 & 0.060127 \\
\hline 1 & -6.731298 & -4.731868 & 0.835803 \\
\hline 6 & 7.308715 & -2.521457 & -1.038320 \\
\hline 1 & 7.883800 & -3.433950 & -1.224352 \\
\hline 1 & 7.209364 & -1.980257 & -1.982521 \\
\hline 1 & 7.879771 & -1.890477 & -0.34943 \\
\hline 6 & 6.127896 & -3.671282 & 0.847897 \\
\hline 1 & 6.749518 & -3.127651 & 1.567686 \\
\hline 1 & 5.174367 & -3.900434 & 1.332212 \\
\hline 1 & 6.634030 & -4.614014 & 0.621583 \\
\hline 6 & -2.300412 & 0.531607 & 3.53741 \\
\hline 1 & -1.431727 & 0.141273 & 3.000515 \\
\hline 1 & -1.942441 & 1.224434 & 4.305173 \\
\hline 1 & -2.802264 & -0.297959 & 4.048184 \\
\hline 6 & -4.436881 & 1.870945 & 3.334981 \\
\hline 1 & -5.105933 & 2.400057 & 2.65034 \\
\hline
\end{tabular}




\begin{tabular}{|c|c|c|c|}
\hline 1 & -5.021985 & 1.102445 & 3.850892 \\
\hline 1 & -4.079773 & 2.581625 & 4.086405 \\
\hline 6 & 4.802517 & 1.105002 & 3.333758 \\
\hline 1 & 5.609930 & 0.371430 & 3.435565 \\
\hline 1 & 5.208859 & 1.974773 & 2.810275 \\
\hline 1 & 4.502017 & 1.418927 & 4.338326 \\
\hline 6 & 3.029175 & -0.675507 & 3.388740 \\
\hline 1 & 3.789347 & -1.427224 & 3.626177 \\
\hline 1 & 2.634399 & -0.302778 & 4.339160 \\
\hline 1 & 2.218899 & -1.154318 & 2.836284 \\
\hline 6 & 4.888670 & 1.603099 & -3.223227 \\
\hline 1 & 5.465557 & 2.250912 & -2.556957 \\
\hline 1 & 5.538584 & 0.778275 & -3.534598 \\
\hline 1 & 4.620955 & 2.176177 & -4.116310 \\
\hline 6 & 2.798082 & 0.203315 & -3.469754 \\
\hline 1 & 1.893723 & -0.157927 & -2.974089 \\
\hline 1 & 2.501469 & 0.780110 & -4.351249 \\
\hline 1 & 3.372782 & -0.661276 & -3.817925 \\
\hline 1 & -2.152189 & -2.667336 & -0.622299 \\
\hline 6 & -2.526122 & -4.657901 & -1.368237 \\
\hline 1 & -3.600439 & -4.469235 & -1.435822 \\
\hline 1 & -2.097263 & -4.508108 & -2.363875 \\
\hline 1 & -2.363118 & -5.697670 & -1.074220 \\
\hline
\end{tabular}

Number of imaginary frequencies=

The smallest frequency is= 1 $-198.9802 \mathrm{~cm}(-1)$

Electronic energy= Zero-point correction= Thermal correction to Energy= Thermal correction to Enthalpy= Thermal correction to Gibbs Free Energy= Sum of electronic and zero-point Energies= Sum of electronic and thermal Energies= Sum of electronic and thermal Enthalpies= Sum of electronic and thermal Free Energies=
$-3160.4389735\left(\mathbf{E}_{\mathrm{sol}}=\mathbf{- 3 1 6 0 . 4 9 0 6 6 5 )}\right.$

1.230454 (Hartree/Particle)

1.297287

1.298231

1.128797

$-3159.208520$

$-3159.141686$

$-3159.140742$

$-3159.310176$

Cartesian Coordinates

$\begin{array}{rrrr}\ldots \ldots \ldots \ldots \ldots & \ldots .019414 & 2.322214 & 1.122607 \\ 6 & 3.332091 & 1.379399 & 0.401549 \\ 6 & 2.014793 & 1.715794 & -0.009537 \\ 6 & 1.377742 & 2.896041 & 0.305308 \\ 6 & 2.069067 & 3.825253 & 1.151325 \\ 1 & 5.046733 & 2.126160 & 1.416829 \\ 6 & -1.705139 & 4.471356 & -1.369756 \\ 6 & -2.661952 & 3.467463 & -1.074283 \\ 6 & -2.317338 & 2.301174 & -0.434612 \\ 6 & -0.970481 & 2.165470 & -0.008635 \\ 6 & -0.001335 & 3.129796 & -0.207824 \\ 6 & -0.353306 & 4.297007 & -0.961762 \\ 1 & -3.694526 & 3.621089 & -1.378098 \\ 6 & 0.219646 & 6.381919 & -2.067908 \\ 6 & 0.600704 & 5.275455 & -1.353102 \\ 1 & 0.964846 & 7.113666 & -2.362979 \\ 1 & 1.642505 & 5.132022 & -1.089402\end{array}$




\begin{tabular}{|c|c|c|c|}
\hline 6 & -1.132838 & 6.574576 & -2.434800 \\
\hline 6 & -2.070257 & 5.635061 & -2.097098 \\
\hline 1 & -1.419461 & 7.457580 & -2.996436 \\
\hline 1 & -3.109179 & 5.756185 & -2.391548 \\
\hline 6 & 2.167320 & 5.876827 & 2.447774 \\
\hline 6 & 1.462458 & 5.007117 & 1.654779 \\
\hline 1 & 1.684357 & 6.771992 & 2.826183 \\
\hline 1 & 0.426312 & 5.213434 & 1.409376 \\
\hline 6 & 3.516120 & 5.615278 & 2.782712 \\
\hline 6 & 4.119511 & 4.469719 & 2.334606 \\
\hline 1 & 4.064250 & 6.315322 & 3.404699 \\
\hline 1 & 5.148139 & 4.243130 & 2.601678 \\
\hline 8 & -0.641954 & 1.015552 & 0.683894 \\
\hline 8 & 1.359345 & 0.802530 & -0.817493 \\
\hline 15 & 0.232450 & -0.108785 & -0.107427 \\
\hline 8 & -0.573997 & -0.757741 & -1.199058 \\
\hline 8 & 0.825592 & -0.986339 & 0.966967 \\
\hline 1 & 0.069647 & -2.064124 & 1.304316 \\
\hline 8 & -0.648842 & -2.821058 & 1.553190 \\
\hline 6 & -1.753476 & -2.200696 & 2.224758 \\
\hline 6 & -3.000578 & -2.822738 & 1.609724 \\
\hline 6 & -2.648647 & -2.941216 & 0.131579 \\
\hline 6 & -1.262588 & -3.569264 & 0.077197 \\
\hline 8 & -0.433062 & -3.221202 & -0.874457 \\
\hline 1 & -1.659832 & -2.377827 & 3.299031 \\
\hline 1 & -1.727191 & -1.119440 & 2.030329 \\
\hline 6 & 3.413247 & 3.541500 & 1.525983 \\
\hline 8 & -1.274238 & -4.857579 & 0.401657 \\
\hline 6 & -0.076738 & -5.695004 & 0.288549 \\
\hline 6 & 0.158081 & -6.052877 & -1.177021 \\
\hline 1 & 0.907581 & -6.847782 & -1.237041 \\
\hline 1 & -0.770580 & -6.420695 & -1.622761 \\
\hline 1 & 0.508399 & -5.192481 & -1.745966 \\
\hline 6 & -0.473019 & -6.935602 & 1.081079 \\
\hline 1 & 0.343293 & -7.662135 & 1.068061 \\
\hline 1 & -0.691704 & -6.669272 & 2.118118 \\
\hline 1 & -1.361584 & -7.398416 & 0.643419 \\
\hline 6 & 1.143836 & -5.027289 & 0.918985 \\
\hline 1 & 1.423093 & -4.108568 & 0.398232 \\
\hline 1 & 0.955957 & -4.794139 & 1.969154 \\
\hline 1 & 1.984749 & -5.725295 & 0.858981 \\
\hline 1 & -0.507935 & -2.211316 & -1.097226 \\
\hline 6 & 3.893052 & 0.036210 & 0.057745 \\
\hline 6 & 4.219884 & -0.266145 & -1.277950 \\
\hline 6 & 4.041160 & -0.940864 & 1.061680 \\
\hline 6 & 4.724064 & -1.529188 & -1.581467 \\
\hline 6 & 4.554176 & -2.190114 & 0.706885 \\
\hline 6 & 4.900495 & -2.505940 & -0.603008 \\
\hline 1 & 4.977959 & -1.757833 & -2.614933 \\
\hline 1 & 4.675021 & -2.950934 & 1.474159 \\
\hline 6 & -3.346295 & 1.238470 & -0.191427 \\
\hline 6 & -3.936862 & 1.114342 & 1.084813 \\
\hline 6 & -3.803920 & 0.446264 & -1.257649 \\
\hline 6 & -5.036739 & 0.274264 & 1.236905 \\
\hline 6 & -4.914477 & -0.384116 & -1.054128 \\
\hline 6 & -5.566574 & -0.461775 & 0.172248 \\
\hline 1 & -5.526936 & 0.214240 & 2.206484 \\
\hline 1 & -5.286109 & -0.961594 & -1.895108 \\
\hline
\end{tabular}




\begin{tabular}{|c|c|c|c|}
\hline 6 & -6.842631 & -1.265463 & 0.391488 \\
\hline 1 & -7.568826 & -0.565147 & 0.827099 \\
\hline 6 & -3.450395 & 1.953105 & 2.257399 \\
\hline 1 & -2.388804 & 2.166151 & 2.097218 \\
\hline 6 & 4.053667 & 0.742631 & -2.404056 \\
\hline & 3.576220 & 1.639635 & -1.998583 \\
\hline 6 & 3.659932 & -0.683589 & 2.512688 \\
\hline 1 & 2.959221 & 0.156897 & 2.528590 \\
\hline 6 & 5.430928 & -3.881613 & -0.958213 \\
\hline & 5.491553 & -4.456862 & -0.025274 \\
\hline & -3.157230 & 0.489848 & -2.635559 \\
\hline & -2.162239 & 0.931166 & -2.52302 \\
\hline 6 & -3.981124 & 1.354871 & -3.598443 \\
\hline 1 & -4.083400 & 2.380478 & -3.235250 \\
\hline 1 & -3.507425 & 1.388610 & -4.584384 \\
\hline 1 & -4.987368 & 0.938333 & -3.719266 \\
\hline 6 & -2.958724 & -0.907509 & -3.23334 \\
\hline 1 & -3.913054 & -1.389791 & -3.472180 \\
\hline 1 & -2.390785 & -0.830172 & -4.165140 \\
\hline 1 & -2.396233 & -1.548610 & -2.551981 \\
\hline 6 & -7.454367 & -1.816186 & -0.895568 \\
\hline 1 & -8.413518 & -2.294461 & -0.679465 \\
\hline 1 & -7.624816 & -1.026781 & -1.632550 \\
\hline 1 & -6.806778 & -2.573304 & -1.352019 \\
\hline 6 & -6.643874 & -2.399081 & 1.406822 \\
\hline 1 & -5.957842 & -3.156330 & 1.012946 \\
\hline 1 & -6.233132 & -2.030670 & 2.351324 \\
\hline 1 & -7.597967 & -2.889450 & 1.622294 \\
\hline 6 & 4.475855 & -4.618532 & -1.903980 \\
\hline 1 & 4.845159 & -5.626400 & -2.118793 \\
\hline 1 & 3.475631 & -4.699450 & -1.469176 \\
\hline 1 & 4.384081 & -4.084839 & -2.855599 \\
\hline 6 & 6.838894 & -3.805248 & -1.557733 \\
\hline 1 & 6.828705 & -3.256897 & -2.505375 \\
\hline 1 & 7.527490 & -3.292333 & -0.881080 \\
\hline 1 & 7.230266 & -4.807820 & -1.756400 \\
\hline 6 & -3.566121 & 1.233181 & 3.603428 \\
\hline 1 & -3.119684 & 0.234806 & 3.564400 \\
\hline 1 & -3.050124 & 1.806959 & 4.378010 \\
\hline 1 & -4.609043 & 1.127724 & 3.919241 \\
\hline 6 & -4.203260 & 3.289491 & 2.301821 \\
\hline 1 & -4.074712 & 3.846462 & 1.369903 \\
\hline 1 & -5.274330 & 3.113293 & 2.449039 \\
\hline 1 & -3.841830 & 3.910790 & 3.12716 \\
\hline 6 & 4.897748 & -0.309674 & 3.33994 \\
\hline 1 & 5.623904 & -1.129854 & 3.321479 \\
\hline 1 & 5.394406 & 0.582746 & 2.951288 \\
\hline 1 & 4.623441 & -0.121315 & 4.382843 \\
\hline 6 & 2.951919 & -1.877935 & 3.162808 \\
\hline 1 & 2.126919 & -2.227698 & 2.54042 \\
\hline 1 & 3.641297 & -2.710996 & 3.336541 \\
\hline 1 & 2.552479 & -1.581496 & 4.137660 \\
\hline 6 & 5.420058 & 1.163938 & -2.957952 \\
\hline 1 & 6.051094 & 1.582013 & -2.168276 \\
\hline 1 & 5.947173 & 0.306939 & -3.39056 \\
\hline 1 & 5.302388 & 1.918166 & -3.742189 \\
\hline 6 & 3.147211 & 0.202329 & -3.515003 \\
\hline 1 & 2.178271 & -0.101608 & -3.11134 \\
\hline
\end{tabular}




$\begin{array}{rrrr}1 & 2.981838 & 0.971370 & -4.275902 \\ 1 & 3.602504 & -0.661196 & -4.011262 \\ 1 & -2.544749 & -1.927779 & -0.279860 \\ 6 & -3.627678 & -3.742846 & -0.718539 \\ 1 & -3.814820 & -4.723473 & -0.273196 \\ 1 & -4.571429 & -3.198427 & -0.802419 \\ 1 & -3.231256 & -3.891022 & -1.727788 \\ 1 & -3.875383 & -2.188288 & 1.767089 \\ 1 & -3.192448 & -3.815731 & 2.031076 \\ - & {[6-7]_{S \_ \text {reanti }}(\mathbf{i v})}\end{array}$

Number of imaginary frequencies $=1$

The smallest frequency is $=\quad-198.7241 \mathrm{~cm}(-1)$

Electronic energy=

$-3160.4387113\left(\mathbf{E}_{\mathrm{sol}}=\mathbf{- 3 1 6 0 . 4 9 0 3 6 6 )}\right.$

Zero-point correction=

1.229982 (Hartree/Particle)

Thermal correction to Energy=

1.296738

Thermal correction to Enthalpy=

1.297682

Thermal correction to Gibbs Free Energy=

1.129531

Sum of electronic and zero-point Energies=

$-3159.208729$

Sum of electronic and thermal Energies=

$-3159.141974$

Sum of electronic and thermal Enthalpies=

$-3159.141029$

Sum of electronic and thermal Free Energies=

$-3159.309180$

Cartesian Coordinates

\begin{tabular}{|c|c|c|c|}
\hline 6 & 3.538748 & 3.054467 & 0.850583 \\
\hline 6 & 2.971764 & 1.961034 & 0.246478 \\
\hline 6 & 1.615471 & 2.072919 & -0.159157 \\
\hline 6 & 0.824519 & 3.178176 & 0.077041 \\
\hline 6 & 1.400509 & 4.270738 & 0.807514 \\
\hline 1 & 4.585436 & 3.020933 & 1.140752 \\
\hline 6 & -2.579729 & 4.111166 & -1.470133 \\
\hline 6 & -3.338935 & 2.993897 & -1.033846 \\
\hline 6 & -2.761911 & 1.953871 & -0.349352 \\
\hline 6 & -1.386366 & 2.077863 & -0.019412 \\
\hline 6 & -0.599768 & 3.158245 & -0.360697 \\
\hline 6 & -1.191652 & 4.188733 & -1.163274 \\
\hline 1 & -4.397397 & 2.953110 & -1.276957 \\
\hline 6 & -1.044895 & 6.237796 & -2.458433 \\
\hline 6 & -0.440260 & 5.268821 & -1.698818 \\
\hline 1 & -0.451838 & 7.051429 & -2.863406 \\
\hline 1 & 0.625853 & 5.314580 & -1.506136 \\
\hline 6 & -2.432559 & 6.182827 & -2.725745 \\
\hline 6 & -3.178397 & 5.138899 & -2.245364 \\
\hline 1 & -2.899081 & 6.960019 & -3.322210 \\
\hline 1 & -4.241026 & 5.070548 & -2.461717 \\
\hline 6 & 1.239653 & 6.428377 & 1.913690 \\
\hline 6 & 0.644980 & 5.397267 & 1.231869 \\
\hline 1 & 0.642663 & 7.277434 & 2.230583 \\
\hline 1 & -0.417069 & 5.432538 & 1.016941 \\
\hline 6 & 2.621251 & 6.393052 & 2.213348 \\
\hline 6 & 3.369471 & 5.306225 & 1.845376 \\
\hline 1 & 3.080657 & 7.218937 & 2.74648 \\
\hline 1 & 4.426610 & 5.251707 & 2.090632 \\
\hline 8 & -0.829210 & 1.047693 & 0.71690 \\
\hline 8 & 1.093381 & 1.010002 & -0.87237 \\
\hline
\end{tabular}




\begin{tabular}{|c|c|c|c|}
\hline 15 & 0.078819 & -0.001780 & -0.123283 \\
\hline 8 & -0.718460 & -0.695721 & -1.193943 \\
\hline 8 & 0.782337 & -0.855565 & 0.904248 \\
\hline 1 & 0.342661 & -2.133595 & 0.969978 \\
\hline 8 & -0.143802 & -3.093341 & 1.057849 \\
\hline 6 & -1.344432 & -2.942509 & 1.831003 \\
\hline 6 & -2.366975 & -3.834423 & 1.141257 \\
\hline 6 & -2.083449 & -3.596320 & -0.335587 \\
\hline 6 & -0.576858 & -3.760854 & -0.488698 \\
\hline 8 & 0.060384 & -3.046934 & -1.387531 \\
\hline 1 & -1.135084 & -3.222523 & 2.866042 \\
\hline 1 & -1.670883 & -1.894078 & 1.793380 \\
\hline 6 & 2.780903 & 4.215698 & 1.152748 \\
\hline 8 & -0.198722 & -5.032105 & -0.380009 \\
\hline 6 & 1.172254 & -5.460061 & -0.664372 \\
\hline 6 & 1.377286 & -5.529918 & -2.175627 \\
\hline 1 & 2.313514 & -6.054860 & -2.387908 \\
\hline 1 & 0.557396 & -6.088652 & -2.636038 \\
\hline 1 & 1.422654 & -4.535324 & -2.617684 \\
\hline 6 & 1.206041 & -6.858772 & -0.058719 \\
\hline 1 & 2.188459 & -7.310956 & -0.216044 \\
\hline 1 & 1.008506 & -6.810697 & 1.015051 \\
\hline 1 & 0.448787 & -7.494029 & -0.525927 \\
\hline 6 & 2.206501 & -4.563120 & 0.012500 \\
\hline 1 & 2.195283 & -3.545279 & -0.382685 \\
\hline 1 & 2.039302 & -4.525210 & 1.091720 \\
\hline 1 & 3.196342 & -4.991982 & -0.173454 \\
\hline 1 & -0.288389 & -2.073100 & -1.400760 \\
\hline 6 & 3.699607 & 0.667523 & 0.067303 \\
\hline 6 & 4.056314 & 0.218960 & -1.219102 \\
\hline 6 & 3.992803 & -0.121132 & 1.195365 \\
\hline 6 & 4.740786 & -0.986990 & -1.343829 \\
\hline 6 & 4.700912 & -1.313337 & 1.019871 \\
\hline 6 & 5.087676 & -1.764042 & -0.237772 \\
\hline 1 & 5.015621 & -1.338045 & -2.337579 \\
\hline 1 & 4.935148 & -1.908915 & 1.896307 \\
\hline 6 & -3.518825 & 0.722165 & 0.034682 \\
\hline 6 & -3.759791 & 0.436619 & 1.390760 \\
\hline 6 & -3.994062 & -0.156904 & -0.964438 \\
\hline 6 & -4.519109 & -0.689735 & 1.727529 \\
\hline 6 & -4.756881 & -1.257931 & -0.576805 \\
\hline 6 & -5.048241 & -1.537367 & 0.759578 \\
\hline 1 & -4.712914 & -0.885988 & 2.778437 \\
\hline 1 & -5.146993 & -1.925266 & -1.343968 \\
\hline 6 & -5.957966 & -2.710935 & 1.090957 \\
\hline 1 & -5.585506 & -3.580587 & 0.528917 \\
\hline 6 & -3.274570 & 1.334402 & 2.520196 \\
\hline 1 & -2.687635 & 2.150551 & 2.090683 \\
\hline 6 & 3.737768 & 1.013888 & -2.475672 \\
\hline 1 & 3.147528 & 1.889661 & -2.190924 \\
\hline 6 & 3.551820 & 0.265298 & 2.601075 \\
\hline 1 & 2.770396 & 1.026186 & 2.510786 \\
\hline 6 & 5.874973 & -3.046839 & -0.450934 \\
\hline 1 & 5.364749 & -3.604057 & -1.249589 \\
\hline 6 & -3.733906 & 0.060579 & -2.449196 \\
\hline 1 & -2.952002 & 0.818036 & -2.549838 \\
\hline 6 & -5.003953 & 0.570373 & -3.143808 \\
\hline 7 & -5.373920 & 1.494360 & -2.691017 \\
\hline
\end{tabular}




\begin{tabular}{|c|c|c|c|}
\hline & & & \\
\hline 1 & -4.813294 & 0.760700 & -4.204361 \\
\hline 1 & -5.803038 & -0.175928 & -3.071288 \\
\hline 6 & -3.227520 & -1.204281 & -3.151810 \\
\hline 1 & -3.970072 & -2.009537 & -3.129591 \\
\hline 1 & -3.020236 & -0.982783 & -4.203236 \\
\hline 1 & -2.303399 & -1.558588 & -2.692005 \\
\hline 6 & -5.983605 & -3.081580 & 2.573340 \\
\hline 1 & -6.576434 & -3.987757 & 2.723295 \\
\hline 1 & -4.981819 & -3.260547 & 2.975875 \\
\hline 1 & -6.445047 & -2.283881 & 3.165054 \\
\hline 6 & -7.385310 & -2.420464 & 0.605014 \\
\hline 1 & -7.788214 & -1.549241 & 1.131536 \\
\hline 1 & -7.408516 & -2.204473 & -0.466000 \\
\hline 1 & -8.042303 & -3.273699 & 0.799463 \\
\hline 6 & 7.294245 & -2.723071 & -0.938581 \\
\hline 1 & 7.854768 & -3.640867 & -1.142320 \\
\hline 1 & 7.278461 & -2.120323 & -1.850256 \\
\hline 1 & 7.831729 & -2.155855 & -0.171621 \\
\hline 6 & 5.941998 & -3.944628 & 0.783398 \\
\hline 1 & 6.536206 & -3.475561 & 1.575118 \\
\hline 1 & 4.948202 & -4.155635 & 1.188764 \\
\hline 1 & 6.421160 & -4.895271 & 0.532888 \\
\hline 6 & -2.364078 & 0.579420 & 3.495001 \\
\hline 1 & -1.480987 & 0.190796 & 2.980725 \\
\hline 1 & -2.025895 & 1.249328 & 4.291611 \\
\hline 1 & -2.893320 & -0.255647 & 3.968119 \\
\hline 6 & -4.463579 & 1.968516 & 3.253415 \\
\hline 1 & -5.103526 & 2.519632 & 2.558472 \\
\hline 1 & -5.077383 & 1.205943 & 3.744034 \\
\hline 1 & -4.112203 & 2.662459 & 4.022974 \\
\hline 6 & 4.719004 & 0.860588 & 3.398803 \\
\hline 1 & 5.522736 & 0.123126 & 3.501875 \\
\hline 1 & 5.139026 & 1.743274 & 2.909694 \\
\hline 1 & 4.393025 & 1.148646 & 4.403225 \\
\hline 6 & 2.943573 & -0.914357 & 3.368547 \\
\hline 1 & 2.158443 & -1.392122 & 2.780227 \\
\hline 1 & 3.700356 & -1.663014 & 3.625615 \\
\hline 1 & 2.510177 & -0.557227 & 4.307982 \\
\hline 6 & 5.025342 & 1.524563 & -3.133748 \\
\hline 1 & 5.604809 & 2.138693 & -2.438443 \\
\hline 1 & 5.659065 & 0.690171 & -3.452817 \\
\hline 1 & 4.792967 & 2.127701 & -4.016934 \\
\hline 6 & 2.900619 & 0.195048 & -3.464224 \\
\hline 1 & 1.977332 & -0.156199 & -2.996806 \\
\hline 1 & 2.636589 & 0.805509 & -4.333304 \\
\hline 1 & 3.458461 & -0.674439 & -3.827275 \\
\hline 1 & -2.301686 & -2.543843 & -0.561616 \\
\hline 6 & -2.835970 & -4.497087 & -1.305518 \\
\hline 1 & -2.655528 & -5.550155 & -1.076520 \\
\hline 1 & -3.909630 & -4.297776 & -1.239261 \\
\hline 1 & -2.517888 & -4.304681 & -2.334600 \\
\hline 1 & -3.386506 & -3.553531 & 1.411668 \\
\hline 1 & -2.203383 & -4.887947 & 1.391872 \\
\hline
\end{tabular}

\section{[6-7] S_si_anti $^{\ddagger}$ (i)}

Number of imaginary frequencies= 1

The smallest frequency is $\quad-179.4056 \mathrm{~cm}(-1)$ 


\begin{tabular}{|c|c|c|c|}
\hline Electro & onic energy= & -3160.4377827 & $=-3160.489505)$ \\
\hline Zero-po & int correction & 1.22932 & cee/Particle) \\
\hline Thermal & correction to & Energy= & 1.295929 \\
\hline Thermal & correction to & Enthalpy= & 1.296873 \\
\hline Thermal & correction to & Gibbs Free Energy= & 1.128993 \\
\hline Sum of & electronic and & zero-point Energies= & -3159.208453 \\
\hline Sum of & electronic and & thermal Energies= & -3159.141854 \\
\hline Sum of & electronic and & thermal Enthalpies= & -3159.140910 \\
\hline Sum of & electronic and & thermal Free Energies= & -3159.308789 \\
\hline
\end{tabular}

Cartesian Coordinates

\begin{tabular}{|c|c|c|c|}
\hline 6 & 2.454822 & 3.842327 & 0.910338 \\
\hline 6 & 2.200874 & 2.633961 & 0.312605 \\
\hline 6 & 0.864548 & 2.384237 & -0.099404 \\
\hline 6 & -0.191422 & 3.241964 & 0.128576 \\
\hline 6 & 0.070174 & 4.446584 & 0.862310 \\
\hline 1 & 3.473476 & 4.095124 & 1.190912 \\
\hline 6 & -3.688487 & 3.261419 & -1.490318 \\
\hline 6 & -4.134672 & 1.977991 & -1.080881 \\
\hline 6 & -3.319613 & 1.120199 & -0.385694 \\
\hline 6 & -2.035076 & 1.598402 & -0.013513 \\
\hline 6 & -1.553311 & 2.850957 & -0.333382 \\
\hline 6 & -2.377142 & 3.696878 & -1.147656 \\
\hline 1 & -5.134030 & 1.655462 & -1.362057 \\
\hline 6 & -2.745684 & 5.726727 & -2.430628 \\
\hline 6 & -1.924826 & 4.942714 & -1.660801 \\
\hline 1 & -2.378039 & 6.672027 & -2.816451 \\
\hline 1 & -0.913091 & 5.266542 & -1.443569 \\
\hline 6 & -4.062716 & 5.311207 & -2.735006 \\
\hline 6 & -4.517790 & 4.102481 & -2.278585 \\
\hline 1 & -4.702271 & 5.943710 & -3.341863 \\
\hline 1 & -5.518893 & 3.758352 & -2.523435 \\
\hline 6 & -0.661586 & 6.481353 & 1.969736 \\
\hline 6 & -0.959043 & 5.330288 & 1.285096 \\
\hline 1 & -1.463691 & 7.139615 & 2.287606 \\
\hline 1 & -1.991745 & 5.081761 & 1.066902 \\
\hline 6 & 0.678968 & 6.814261 & 2.273475 \\
\hline 6 & 1.690395 & 5.966043 & 1.906558 \\
\hline 1 & 0.900295 & 7.730987 & 2.810029 \\
\hline 1 & 2.723451 & 6.193451 & 2.155262 \\
\hline 8 & -1.259764 & 0.750253 & 0.752425 \\
\hline 8 & 0.635972 & 1.213032 & -0.801096 \\
\hline 15 & -0.019308 & -0.006470 & 0.037108 \\
\hline 8 & -0.487934 & -1.006837 & -0.991773 \\
\hline 8 & 0.856923 & -0.490385 & 1.158013 \\
\hline 6 & -0.043767 & -4.163804 & 1.319019 \\
\hline 6 & -0.963545 & -4.942165 & 0.376063 \\
\hline 6 & -1.065472 & -4.057001 & -0.872140 \\
\hline 6 & 1.129647 & -3.738605 & 0.433742 \\
\hline 8 & 1.854868 & -2.682769 & 0.681337 \\
\hline 1 & 0.382173 & -4.824066 & 2.080771 \\
\hline 6 & 1.414326 & 4.759651 & 1.211679 \\
\hline 8 & 1.811517 & -4.816794 & 0.034082 \\
\hline 6 & 3.058655 & -4.711677 & -0.724608 \\
\hline 6 & 3.275614 & -6.152427 & -1.175383 \\
\hline 1 & 4.200179 & -6.228739 & -1.752921 \\
\hline
\end{tabular}




\begin{tabular}{|c|c|c|c|}
\hline 1 & 3.347714 & -6.813842 & -0.307956 \\
\hline 1 & 2.441420 & -6.483850 & -1.799190 \\
\hline 6 & 4.199051 & -4.262474 & 0.188053 \\
\hline$\perp$ & 5.152634 & -4.435678 & -0.322095 \\
\hline & 4.118320 & -3.203028 & 0.436098 \\
\hline & 4.191861 & -4.848659 & 1.111393 \\
\hline 6 & 2.908680 & -3.791471 & -1.932718 \\
\hline 1 & 2.052412 & -4.087035 & -2.542075 \\
\hline 1 & 2.787672 & -2.747989 & -1.631420 \\
\hline$\perp$ & 3.814503 & -3.874337 & -2.541213 \\
\hline & -0.038756 & -2.293889 & -0.991484 \\
\hline 6 & 3.257499 & 1.590886 & 0.12527 \\
\hline 6 & 3.737936 & 1.290523 & -1.16006 \\
\hline 6 & 3.751145 & 0.890358 & 1.247263 \\
\hline 6 & 4.750114 & 0.337648 & -1.303712 \\
\hline 6 & 4.763837 & -0.047329 & 1.052492 \\
\hline 6 & 5.286805 & -0.332245 & -0.208601 \\
\hline 1 & 5.122384 & 0.120889 & -2.301721 \\
\hline 1 & 5.158519 & -0.588656 & 1.910301 \\
\hline 6 & -3.713979 & -0.285072 & -0.061865 \\
\hline 6 & -3.882163 & -0.689706 & 1.275118 \\
\hline 6 & -3.897050 & -1.216643 & -1.107609 \\
\hline 6 & -4.255897 & -2.008983 & 1.547678 \\
\hline 6 & -4.291203 & -2.514154 & -0.783129 \\
\hline 6 & -4.471648 & -2.936977 & 0.534090 \\
\hline 1 & -4.380846 & -2.307822 & 2.585040 \\
\hline 1 & -4.448165 & -3.234927 & -1.584941 \\
\hline 6 & -4.932400 & -4.360668 & 0.797986 \\
\hline 1 & -4.382831 & -5.009266 & 0.100057 \\
\hline 6 & -3.712005 & 0.263051 & 2.449605 \\
\hline 1 & -3.382414 & 1.232504 & 2.066837 \\
\hline 6 & 3.206248 & 1.978913 & -2.407174 \\
\hline 1 & 2.380851 & 2.634753 & -2.115783 \\
\hline 6 & 3.211132 & 1.114501 & 2.653222 \\
\hline 1 & 2.221318 & 1.571749 & 2.559299 \\
\hline 6 & 6.444482 & -1.306789 & -0.331546 \\
\hline 1 & 6.310700 & -2.064967 & 0.451761 \\
\hline 6 & -3.687236 & -0.869218 & -2.576059 \\
\hline 1 & -3.216104 & 0.115435 & -2.631451 \\
\hline 6 & -5.033168 & -0.801597 & -3.309159 \\
\hline 1 & -5.708159 & -0.073274 & -2.85055 \\
\hline 1 & -4.887552 & -0.523396 & -4.357413 \\
\hline 1 & -5.532938 & -1.776285 & -3.283816 \\
\hline 6 & -2.745235 & -1.852018 & -3.281134 \\
\hline 1 & -3.157495 & -2.867054 & -3.30256 \\
\hline 1 & -2.596165 & -1.539795 & -4.31943 \\
\hline 1 & -1.771500 & -1.871302 & -2.787075 \\
\hline 6 & -4.651558 & -4.850007 & 2.218091 \\
\hline 1 & -4.883659 & -5.915050 & 2.30391 \\
\hline 1 & -3.604352 & -4.702484 & 2.50103 \\
\hline 1 & -5.273481 & -4.319704 & 2.94667 \\
\hline 6 & -6.427477 & -4.495854 & 0.477711 \\
\hline 1 & -7.011455 & -3.848289 & 1.139794 \\
\hline 1 & -6.639747 & -4.200236 & -0.55305 \\
\hline 1 & -6.765470 & -5.527037 & 0.619669 \\
\hline 6 & 6.498683 & -2.028578 & -1.677261 \\
\hline 1 & 7.252802 & -2.821094 & -1.652348 \\
\hline 1 & 5.531582 & -2.475096 & -1.925 \\
\hline
\end{tabular}




\begin{tabular}{|c|c|c|c|}
\hline & & & \\
\hline 1 & 6.773683 & -1.342647 & -2.485260 \\
\hline 6 & 7.768376 & -0.579417 & -0.059448 \\
\hline 1 & 7.927387 & 0.201314 & -0.810690 \\
\hline 1 & 7.760745 & -0.102036 & 0.924185 \\
\hline 1 & 8.613037 & -1.274402 & -0.102288 \\
\hline 6 & -2.643980 & -0.232745 & 3.430689 \\
\hline 1 & -1.679388 & -0.348295 & 2.930003 \\
\hline 1 & -2.522386 & 0.483869 & 4.248829 \\
\hline 1 & -2.927885 & -1.194485 & 3.872582 \\
\hline 6 & -5.053704 & 0.486554 & 3.158951 \\
\hline 1 & -5.807983 & 0.862760 & 2.462006 \\
\hline 1 & -5.430538 & -0.445923 & 3.591937 \\
\hline 1 & -4.940574 & 1.212099 & 3.970082 \\
\hline 6 & 4.119980 & 2.061645 & 3.448497 \\
\hline 1 & 5.123771 & 1.631912 & 3.539061 \\
\hline 1 & 4.217534 & 3.038619 & 2.969778 \\
\hline 1 & 3.724318 & 2.215090 & 4.457509 \\
\hline 6 & 3.036039 & -0.192891 & 3.434624 \\
\hline 1 & 2.499500 & -0.936707 & 2.844228 \\
\hline 1 & 4.000957 & -0.613118 & 3.737566 \\
\hline 1 & 2.467229 & 0.000720 & 4.349078 \\
\hline 6 & 4.285806 & 2.860541 & -3.046156 \\
\hline 1 & 4.656666 & 3.603329 & -2.333989 \\
\hline 1 & 5.138456 & 2.258939 & -3.378285 \\
\hline 1 & 3.885792 & 3.386980 & -3.918300 \\
\hline 6 & 2.650756 & 0.963089 & -3.411382 \\
\hline 1 & 1.872731 & 0.350013 & -2.949335 \\
\hline 1 & 2.217767 & 1.479654 & -4.273573 \\
\hline 1 & 3.440266 & 0.301029 & -3.782597 \\
\hline 1 & -1.872448 & -3.323099 & -0.780288 \\
\hline 1 & -1.949928 & -5.107710 & 0.818955 \\
\hline 1 & -0.521130 & -5.910100 & 0.131232 \\
\hline 8 & 0.176511 & -3.343643 & -0.960657 \\
\hline 1 & 1.373801 & -1.785478 & 0.939096 \\
\hline 1 & -1.206663 & -4.633532 & -1.789740 \\
\hline 6 & -0.762604 & -2.991441 & 1.980206 \\
\hline 1 & -1.544695 & -3.374463 & 2.642185 \\
\hline 1 & -0.084778 & -2.372742 & 2.572037 \\
\hline 1 & -1.256595 & -2.345286 & 1.244307 \\
\hline
\end{tabular}

\section{$[6-7]_{S \_ \text {si_anti }}^{\ddagger}$ (ii)}

Number of imaginary frequencies $=1$

The smallest frequency is $\quad-187.4021 \mathrm{~cm}(-1)$

Electronic energy $=\quad-3160.4376342 \quad\left(\mathbf{E}_{\text {sol }}=\mathbf{- 3 1 6 0 . 4 9 0 0 3 3 )}\right.$

Zero-point correction= 1.230877 (Hartree/Particle)

$\begin{array}{ll}\text { Thermal correction to Energy }= & 1.296969\end{array}$

Thermal correction to Enthalpy= 1.297913

Thermal correction to Gibbs Free Energy= 1.133342

Sum of electronic and zero-point Energies= $\quad$-3159.206757

Sum of electronic and thermal Energies= $\quad-3159.140665$

Sum of electronic and thermal Enthalpies= $\quad-3159.139721$

Sum of electronic and thermal Free Energies= $\quad-3159.304292$

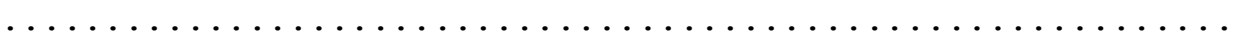

Cartesian Coordinates
6
3.200940
3.270236
0.981495 


\begin{tabular}{|c|c|c|c|}
\hline 6 & 2.741485 & 2.159365 & 0.320702 \\
\hline 6 & 1.367824 & 2.138980 & -0.040589 \\
\hline 6 & 0.480704 & 3.154279 & 0.247928 \\
\hline 6 & 0.949106 & 4.254220 & 1.040634 \\
\hline 1 & 4.257153 & 3.346461 & 1.224123 \\
\hline 6 & -2.917963 & 3.928378 & -1.379806 \\
\hline 6 & -3.611649 & 2.740842 & -1.032631 \\
\hline 6 & -2.991563 & 1.709753 & -0.372923 \\
\hline 6 & -1.639796 & 1.899835 & 0.019186 \\
\hline 6 & -0.922989 & 3.050192 & -0.235400 \\
\hline 6 & -1.552393 & 4.081395 & -1.009251 \\
\hline 1 & -4.654315 & 2.637303 & -1.322870 \\
\hline 6 & -1.503749 & 6.209211 & -2.180545 \\
\hline 6 & -0.860541 & 5.241321 & -1.451927 \\
\hline 1 & -0.954807 & 7.084780 & -2.511895 \\
\hline 1 & 0.191543 & 5.351664 & -1.214936 \\
\hline 6 & -2.872471 & 6.075061 & -2.510050 \\
\hline 6 & -3.558820 & 4.955046 & -2.122125 \\
\hline 1 & -3.370020 & 6.852863 & -3.079971 \\
\hline 1 & -4.604930 & 4.825541 & -2.385726 \\
\hline 6 & 0.578243 & 6.310333 & 2.279097 \\
\hline 6 & 0.086200 & 5.270898 & 1.531269 \\
\hline 1 & -0.099097 & 7.072346 & 2.650850 \\
\hline 1 & -0.975004 & 5.211329 & 1.315828 \\
\hline 6 & 1.957993 & 6.394388 & 2.577642 \\
\hline 6 & 2.810405 & 5.415225 & 2.139707 \\
\hline 1 & 2.333688 & 7.225692 & 3.165234 \\
\hline 1 & 3.869378 & 5.452469 & 2.380259 \\
\hline 8 & -1.051973 & 0.886237 & 0.751570 \\
\hline 8 & 0.926475 & 1.050425 & -0.775147 \\
\hline 15 & 0.059632 & -0.054415 & 0.031093 \\
\hline 8 & -0.566140 & -0.946462 & -1.013667 \\
\hline 8 & 0.832174 & -0.702043 & 1.147069 \\
\hline 6 & -1.041944 & -3.978502 & 1.479609 \\
\hline 6 & -2.134385 & -4.509185 & 0.550343 \\
\hline 6 & -1.969817 & -3.692387 & -0.736579 \\
\hline 6 & 0.211497 & -3.941391 & 0.605042 \\
\hline 8 & 1.195766 & -3.110649 & 0.818711 \\
\hline 1 & -0.824815 & -4.698193 & 2.274881 \\
\hline 6 & 2.329660 & 4.317684 & 1.379131 \\
\hline 8 & 0.566791 & -5.188587 & 0.282775 \\
\hline 6 & 1.812451 & -5.492342 & -0.420842 \\
\hline 6 & 1.599756 & -6.943858 & -0.837359 \\
\hline 1 & 2.481948 & -7.313971 & -1.365724 \\
\hline 1 & 1.428924 & -7.569940 & 0.042414 \\
\hline 1 & 0.732965 & -7.025402 & -1.498352 \\
\hline 6 & 2.989276 & -5.384326 & 0.545864 \\
\hline 1 & 3.879884 & -5.814877 & 0.076938 \\
\hline 1 & 3.196759 & -4.346153 & 0.806170 \\
\hline 1 & 2.773500 & -5.947144 & 1.458441 \\
\hline 6 & 1.999492 & -4.608582 & -1.650381 \\
\hline 1 & 1.128317 & -4.667918 & -2.306124 \\
\hline 1 & 2.163156 & -3.565083 & -1.374449 \\
\hline 1 & 2.876242 & -4.964958 & -2.200167 \\
\hline 1 & -0.481429 & -2.313088 & -0.931457 \\
\hline 6 & 3.639955 & 1.020490 & -0.043809 \\
\hline 6 & 4.045947 & 0.857355 & -1.378430 \\
\hline 6 & 4.083645 & 0.122321 & 0.950091 \\
\hline
\end{tabular}




\begin{tabular}{|c|c|c|c|}
\hline 6 & 4.934462 & -0.173342 & -1.692056 \\
\hline 6 & 4.973317 & -0.887768 & 0.586952 \\
\hline 6 & 5.420457 & -1.045881 & -0.725053 \\
\hline L & 5.261070 & -0.296463 & -2.723032 \\
\hline & 5.325707 & -1.575990 & 1.351707 \\
\hline & -3.689473 & 0.423382 & -0.074668 \\
\hline & -3.977613 & 0.073294 & 1.261575 \\
\hline & -4.072319 & -0.430377 & -1.125507 \\
\hline 6 & -4.650747 & -1.119594 & 1.513058 \\
\hline & -4.778934 & -1.600062 & -0.821370 \\
\hline & -5.071496 & -1.968740 & 0.487993 \\
\hline 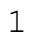 & -4.872731 & -1.394249 & 2.54394 \\
\hline ] & -5.086461 & -2.245322 & -1.640212 \\
\hline 6 & -5.871379 & -3.213239 & 0.835627 \\
\hline 1 & -5.389982 & -3.670897 & 1.711996 \\
\hline 6 & -3.636942 & 0.980565 & 2.434881 \\
\hline 1 & -3.099673 & 1.853162 & 2.053787 \\
\hline 6 & 3.563652 & 1.769384 & -2.495481 \\
\hline 1 & 2.866695 & 2.499014 & -2.072888 \\
\hline 6 & 3.629887 & 0.232797 & 2.399408 \\
\hline 1 & 2.683925 & 0.783117 & 2.409567 \\
\hline 6 & 6.407773 & -2.138336 & -1.088978 \\
\hline 1 & 6.590746 & -2.063070 & -2.168464 \\
\hline 6 & -3.761930 & -0.135733 & -2.587606 \\
\hline 1 & -3.094721 & 0.729094 & -2.627814 \\
\hline 6 & -5.045575 & 0.211530 & -3.352060 \\
\hline 1 & -5.570702 & 1.055117 & -2.894954 \\
\hline 1 & -4.817461 & 0.469549 & -4.390600 \\
\hline 1 & -5.732888 & -0.641384 & -3.359790 \\
\hline 6 & -3.028451 & -1.297217 & -3.269005 \\
\hline 1 & -3.631801 & -2.211924 & -3.270971 \\
\hline 1 & -2.817818 & -1.041895 & -4.312126 \\
\hline 1 & -2.078746 & -1.496907 & -2.767994 \\
\hline 6 & -7.298152 & -2.819815 & 1.244662 \\
\hline 1 & -7.876534 & -3.700670 & 1.539701 \\
\hline 1 & -7.290877 & -2.114317 & 2.079571 \\
\hline 1 & -7.807974 & -2.338815 & 0.403646 \\
\hline 6 & -5.914348 & -4.255277 & -0.281371 \\
\hline 1 & -6.497721 & -3.892155 & -1.133933 \\
\hline 1 & -4.912877 & -4.509765 & -0.643659 \\
\hline 1 & -6.391661 & -5.171943 & 0.075403 \\
\hline 6 & 7.745809 & -1.943107 & -0.368031 \\
\hline 1 & 8.468268 & -2.704330 & -0.678768 \\
\hline 1 & 8.167547 & -0.957219 & -0.580328 \\
\hline 1 & 7.615860 & -2.026456 & 0.71605 \\
\hline 6 & 5.836439 & -3.530730 & -0.803574 \\
\hline 1 & 5.674862 & -3.672455 & 0.270425 \\
\hline 1 & 4.874993 & -3.670281 & -1.305795 \\
\hline 1 & 6.525524 & -4.310664 & -1.143538 \\
\hline 6 & -2.725152 & 0.291350 & 3.45444 \\
\hline 1 & -1.781343 & -0.007301 & 2.991442 \\
\hline 1 & -2.501500 & 0.973849 & 4.280144 \\
\hline 1 & -3.205593 & -0.596623 & 3.88088 \\
\hline 6 & -4.920662 & 1.493195 & 3.10039 \\
\hline 1 & -5.561591 & 2.004105 & 2.376199 \\
\hline 1 & -5.495102 & 0.668345 & 3.53529 \\
\hline 1 & -4.679959 & 2.194651 & 3.904953 \\
\hline 6 & 4.660731 & 0.998486 & 3.24020 \\
\hline
\end{tabular}




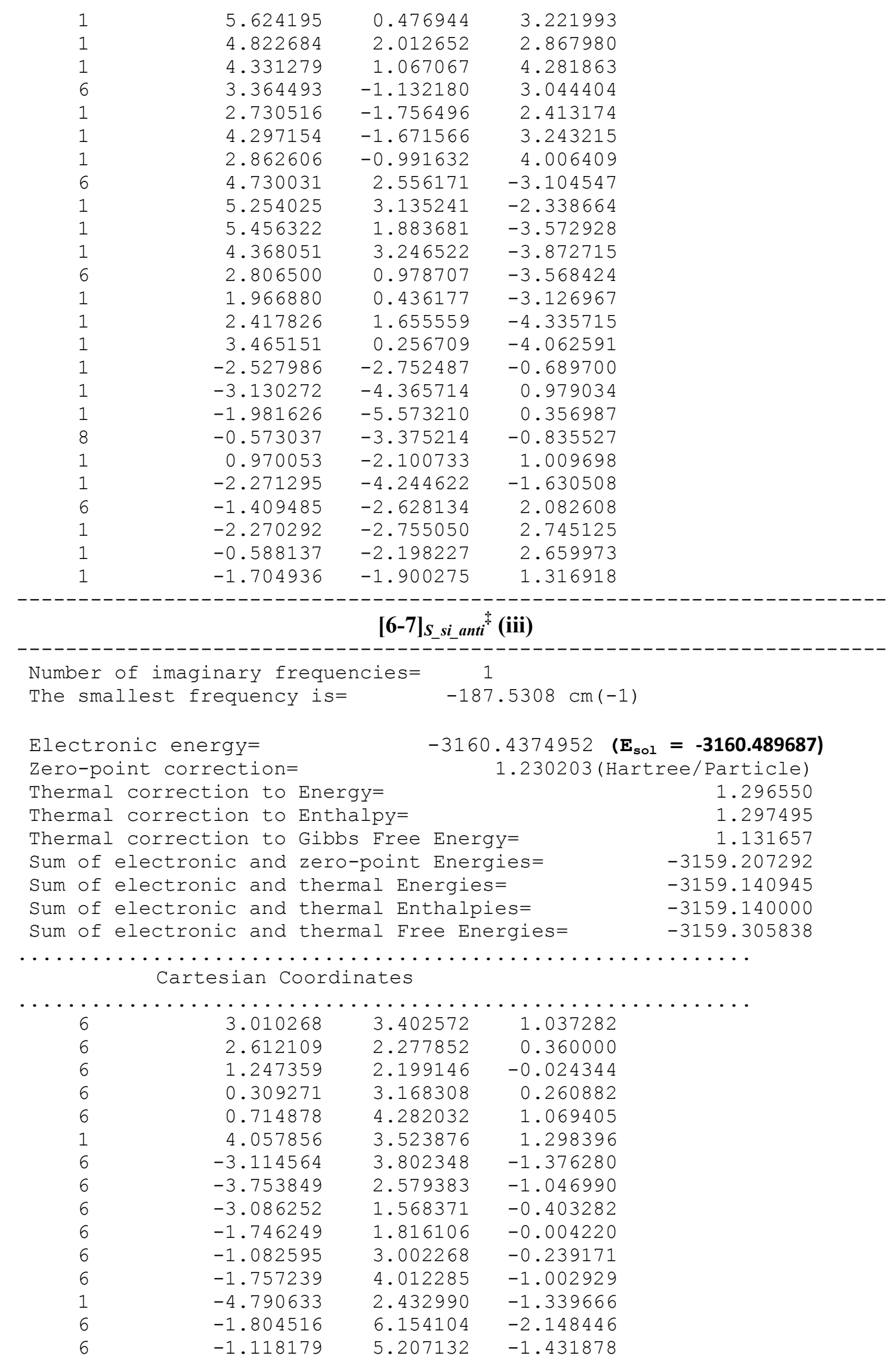




\begin{tabular}{|c|c|c|c|}
\hline 1 & -1.295343 & 7.056843 & -2.470254 \\
\hline 1 & -0.071926 & 5.361419 & -1.193481 \\
\hline 6 & -3.166397 & 5.963608 & -2.478231 \\
\hline 6 & -3.801619 & 4.809024 & -2.104430 \\
\hline 1 & -3.698534 & 6.725728 & -3.037971 \\
\hline 1 & -4.841197 & 4.635980 & -2.369061 \\
\hline 6 & 0.232885 & 6.309998 & 2.316238 \\
\hline 6 & -0.200875 & 5.253458 & 1.556652 \\
\hline 1 & -0.483695 & 7.036850 & 2.684900 \\
\hline 1 & -1.255597 & 5.144687 & 1.329546 \\
\hline 6 & 1.603414 & 6.457096 & 2.631816 \\
\hline 6 & 2.505722 & 5.520913 & 2.200691 \\
\hline 1 & 1.932249 & 7.302161 & 3.227741 \\
\hline 1 & 3.558724 & 5.606182 & 2.454867 \\
\hline 8 & -1.117306 & 0.820775 & 0.718303 \\
\hline 8 & 0.870016 & 1.101066 & -0.779048 \\
\hline 15 & 0.043098 & -0.055668 & -0.004438 \\
\hline 8 & -0.525185 & -0.954637 & -1.075649 \\
\hline 8 & 0.829166 & -0.687371 & 1.111525 \\
\hline 6 & -0.917140 & -4.064304 & 1.259244 \\
\hline 6 & -1.899142 & -4.660326 & 0.248912 \\
\hline 6 & -1.681116 & -3.842594 & -1.031489 \\
\hline 6 & 0.393460 & -3.943054 & 0.481651 \\
\hline 8 & 1.314743 & -3.069442 & 0.779188 \\
\hline 1 & -0.714417 & -4.773767 & 2.067680 \\
\hline 6 & 2.086303 & 4.406457 & 1.427758 \\
\hline 8 & 0.835050 & -5.162149 & 0.158835 \\
\hline 6 & 2.137015 & -5.385180 & -0.469666 \\
\hline 6 & 2.029602 & -6.839096 & -0.917273 \\
\hline 1 & 2.961499 & -7.153927 & -1.393520 \\
\hline 1 & 1.838201 & -7.487048 & -0.057842 \\
\hline 1 & 1.211506 & -6.956353 & -1.632681 \\
\hline 6 & 3.247136 & -5.229544 & 0.567414 \\
\hline 1 & 4.182708 & -5.617361 & 0.152032 \\
\hline 1 & 3.392434 & -4.184806 & 0.842748 \\
\hline 1 & 3.002417 & -5.806192 & 1.463831 \\
\hline 6 & 2.348504 & -4.471307 & -1.672605 \\
\hline 1 & 1.523733 & -4.566922 & -2.382021 \\
\hline 1 & 2.434952 & -3.425485 & -1.371256 \\
\hline 1 & 3.275981 & -4.769139 & -2.171415 \\
\hline 1 & -0.326698 & -2.316531 & -1.045170 \\
\hline 6 & 3.569201 & 1.185521 & 0.002639 \\
\hline 6 & 4.000246 & 1.047049 & -1.327188 \\
\hline 6 & 4.049633 & 0.311739 & 1.001029 \\
\hline 6 & 4.955122 & 0.073574 & -1.628503 \\
\hline 6 & 5.005037 & -0.640615 & 0.650329 \\
\hline 6 & 5.481838 & -0.767189 & -0.654519 \\
\hline 1 & 5.303915 & -0.028070 & -2.654517 \\
\hline 1 & 5.386751 & -1.307482 & 1.419797 \\
\hline 6 & -3.717128 & 0.242385 & -0.126200 \\
\hline 6 & -4.025820 & -0.125377 & 1.196242 \\
\hline 6 & -4.005454 & -0.636664 & -1.191075 \\
\hline 6 & -4.639975 & -1.357172 & 1.434860 \\
\hline 6 & -4.625336 & -1.852664 & -0.900267 \\
\hline 6 & -4.950204 & -2.236454 & 0.401367 \\
\hline 1 & -4.878308 & -1.627930 & 2.460381 \\
\hline 1 & -4.865128 & -2.532511 & -1.717783 \\
\hline 6 & -5.681017 & -3.548476 & 0.628694 \\
\hline
\end{tabular}




\begin{tabular}{|c|c|c|c|}
\hline 1 & -5.269426 & -4.274038 & -0.087388 \\
\hline 6 & -3.749900 & 0.787662 & 2.381355 \\
\hline 1 & -3.251822 & 1.689782 & 2.015018 \\
\hline 6 & 3.474377 & 1.926398 & -2.450943 \\
\hline 1 & 2.729347 & 2.611640 & -2.036210 \\
\hline 6 & 3.564191 & 0.384656 & 2.442686 \\
\hline 1 & 2.594801 & 0.892659 & 2.441837 \\
\hline 6 & 6.546801 & -1.789980 & -1.000985 \\
\hline 1 & 6.734491 & -1.710611 & -2.079390 \\
\hline 6 & -3.701646 & -0.294726 & -2.644463 \\
\hline 1 & -3.082938 & 0.606337 & -2.659223 \\
\hline 6 & -5.003738 & 0.004335 & -3.399051 \\
\hline 1 & -5.572406 & 0.805532 & -2.918576 \\
\hline 1 & -4.792200 & 0.302463 & -4.430392 \\
\hline 1 & -5.643837 & -0.884296 & -3.428172 \\
\hline 6 & -2.908223 & -1.391563 & -3.362971 \\
\hline 1 & -3.456228 & -2.340294 & -3.387020 \\
\hline 1 & -2.726004 & -1.094580 & -4.400434 \\
\hline 1 & -1.941802 & -1.547116 & -2.878788 \\
\hline 6 & -5.504888 & -4.120275 & 2.035548 \\
\hline 1 & -5.941769 & -5.120883 & 2.094748 \\
\hline 1 & -4.449798 & -4.189294 & 2.320017 \\
\hline 1 & -6.012281 & -3.498329 & 2.780040 \\
\hline 6 & -7.172735 & -3.376043 & 0.309813 \\
\hline 1 & -7.617777 & -2.645062 & 0.992745 \\
\hline 1 & -7.319436 & -3.013661 & -0.711122 \\
\hline 1 & -7.708413 & -4.323726 & 0.422778 \\
\hline 6 & 7.860231 & -1.492634 & -0.269322 \\
\hline 1 & 8.638828 & -2.202452 & -0.565839 \\
\hline 1 & 8.211688 & -0.480720 & -0.487038 \\
\hline 1 & 7.724896 & -1.575460 & 0.814154 \\
\hline 6 & 6.075153 & -3.217628 & -0.707944 \\
\hline 1 & 5.903096 & -3.358735 & 0.364538 \\
\hline 1 & 5.137340 & -3.434281 & -1.227008 \\
\hline 1 & 6.827092 & -3.947500 & -1.025052 \\
\hline 6 & -2.813513 & 0.128449 & 3.399263 \\
\hline 1 & -1.860705 & -0.134661 & 2.933094 \\
\hline 1 & -2.613336 & 0.815147 & 4.227515 \\
\hline 1 & -3.261351 & -0.778419 & 3.821628 \\
\hline 6 & -5.062273 & 1.228562 & 3.041276 \\
\hline 1 & -5.721040 & 1.717470 & 2.317900 \\
\hline 1 & -5.600095 & 0.371910 & 3.461183 \\
\hline 1 & -4.862500 & 1.930337 & 3.856665 \\
\hline 6 & 4.543309 & 1.184967 & 3.31230 \\
\hline 1 & 5.530425 & 0.709409 & 3.305612 \\
\hline 1 & 4.664127 & 2.210456 & 2.955561 \\
\hline 1 & 4.192574 & 1.224622 & 4.348450 \\
\hline 6 & 3.346457 & -1.000007 & 3.063617 \\
\hline 1 & 2.738772 & -1.635398 & 2.417618 \\
\hline 1 & 4.297111 & -1.508240 & 3.25909 \\
\hline 1 & 2.833829 & -0.894413 & 4.024473 \\
\hline 6 & 4.595222 & 2.784467 & -3.049251 \\
\hline 1 & 5.069045 & 3.400973 & -2.27982 \\
\hline 1 & 5.370799 & 2.159408 & -3.503778 \\
\hline 1 & 4.199648 & 3.445794 & -3.826283 \\
\hline 6 & 2.777559 & 1.089388 & -3.529239 \\
\hline 1 & 1.964268 & 0.501831 & -3.09585 \\
\hline 1 & 2.361186 & 1.740289 & -4.30446 \\
\hline
\end{tabular}




\begin{tabular}{rrrr}
1 & 3.482259 & 0.404137 & -4.011926 \\
1 & -2.332074 & -2.964487 & -1.058339 \\
1 & -2.933735 & -4.571255 & 0.592527 \\
1 & -1.672365 & -5.714962 & 0.080276 \\
8 & -0.320993 & -3.384688 & -1.001407 \\
1 & 1.029984 & -2.072280 & 0.966246 \\
1 & -1.832923 & -4.431894 & -1.939105 \\
6 & -1.409594 & -2.741968 & 1.836395 \\
1 & -2.295072 & -2.923887 & 2.451906 \\
1 & -0.650856 & -2.260340 & 2.456874 \\
1 & -1.712029 & -2.036175 & 1.052766 \\
\hline
\end{tabular}

\begin{tabular}{|c|c|c|c|}
\hline \multicolumn{4}{|c|}{$\begin{array}{l}\text { Number of imaginary frequencies }= \\
\text { The smallest frequency is }=\end{array}$} \\
\hline Electronic energy= & & \multicolumn{2}{|c|}{$-3160.4379345 \quad\left(E_{\text {sol }}=-\mathbf{3 1 6 0 . 4 8 9 9 0 4}\right)$} \\
\hline Zero-point correction & & 1.229420 & (Hartree/Particle) \\
\hline Thermal correction to & Energy= & & 1.295971 \\
\hline Thermal correction to & Enthalpy $=$ & & 915 \\
\hline Thermal correction to & Gibbs Fre & ee Energy= & 1.129559 \\
\hline Sum of electronic and & zero-poir & nt Energies= & -3159.208515 \\
\hline Sum of electronic and & thermal $\mathrm{E}$ & Energies= & -3159.141964 \\
\hline Sum of electronic and & thermal $\mathrm{E}$ & Enthalpies= & -3159.141019 \\
\hline Sum of electronic and & thermal $\mathrm{E}$ & Free Energies= & -3159.308375 \\
\hline
\end{tabular}

Cartesian Coordinates

$\begin{array}{rrrr}\ldots \ldots \ldots \\ 6 & 2.579139 & 3.786319 & 0.854538 \\ 6 & 2.286278 & 2.577684 & 0.274885 \\ 6 & 0.939416 & 2.358515 & -0.120600 \\ 6 & -0.089259 & 3.250381 & 0.101531 \\ 6 & 0.212178 & 4.456363 & 0.817448 \\ 1 & 3.606913 & 4.014485 & 1.122942 \\ 6 & -3.601275 & 3.356499 & -1.481468 \\ 6 & -4.082697 & 2.093803 & -1.048765 \\ 6 & -3.286510 & 1.220437 & -0.351164 \\ 6 & -1.983091 & 1.662991 & -0.001195 \\ 6 & -1.466109 & 2.894900 & -0.344021 \\ 6 & -2.272584 & 3.755278 & -1.160657 \\ 1 & -5.095409 & 1.799353 & -1.312991 \\ 6 & -2.595029 & 5.779789 & -2.464339 \\ 6 & -1.788841 & 4.980228 & -1.694931 \\ 1 & -2.203938 & 6.708959 & -2.865977 \\ 1 & -0.765094 & 5.275454 & -1.493514 \\ 6 & -3.928024 & 5.401211 & -2.747334 \\ 6 & -4.414198 & 4.212936 & -2.270067 \\ 1 & -4.555701 & 6.045619 & -3.354061 \\ 1 & -5.428594 & 3.897210 & -2.497901 \\ 6 & -0.455261 & 6.522551 & 1.906907 \\ 6 & -0.789300 & 5.372440 & 1.237892 \\ 1 & -1.236421 & 7.205730 & 2.224426 \\ 1 & -1.830001 & 5.148924 & 1.031133 \\ 6 & 0.896326 & 6.822187 & 2.195916 \\ 6 & 1.881370 & 5.942573 & 1.831260 \\ 1 & 1.146633 & 7.738243 & 2.720747 \\ 1 & 2.922046 & 6.144841 & 2.069627 \\ & & & \end{array}$




\begin{tabular}{|c|c|c|c|}
\hline 8 & -1.228311 & 0.803436 & 0.771822 \\
\hline 8 & 0.671383 & 1.181454 & -0.798851 \\
\hline 15 & -0.013747 & -0.002714 & 0.066238 \\
\hline 8 & -0.514880 & -1.010505 & -0.940126 \\
\hline 8 & 0.851910 & -0.490364 & 1.193623 \\
\hline 6 & -0.143068 & -4.139165 & 1.454731 \\
\hline 6 & -1.108105 & -4.906706 & 0.549690 \\
\hline 6 & -1.219547 & -4.037184 & -0.709217 \\
\hline 6 & 1.018190 & -3.763514 & 0.532417 \\
\hline 8 & 1.776323 & -2.721965 & 0.741881 \\
\hline 1 & 0.282585 & -4.797310 & 2.218347 \\
\hline 6 & 1.567106 & 4.735904 & 1.152969 \\
\hline 8 & 1.664026 & -4.867446 & 0.142347 \\
\hline 6 & 2.894734 & -4.810191 & -0.647517 \\
\hline 6 & 3.073930 & -6.267714 & -1.059186 \\
\hline 1 & 3.979578 & -6.377581 & -1.660900 \\
\hline 1 & 3.160953 & -6.903633 & -0.174198 \\
\hline 1 & 2.216302 & -6.603336 & -1.647951 \\
\hline 6 & 4.066530 & -4.356110 & 0.222451 \\
\hline 1 & 5.003370 & -4.575601 & -0.300775 \\
\hline 1 & 4.020147 & -3.286009 & 0.430810 \\
\hline 1 & 4.064247 & -4.905524 & 1.168164 \\
\hline 6 & 2.731096 & -3.923709 & -1.878542 \\
\hline 1 & 1.861187 & -4.227761 & -2.464094 \\
\hline 1 & 2.624258 & -2.871500 & -1.603938 \\
\hline 1 & 3.624880 & -4.031445 & -2.500869 \\
\hline 1 & -0.129535 & -2.313188 & -0.904638 \\
\hline 6 & 3.312034 & 1.504884 & 0.084916 \\
\hline 6 & 3.762709 & 1.177588 & -1.204998 \\
\hline 6 & 3.805189 & 0.803493 & 1.206238 \\
\hline 6 & 4.743848 & 0.194120 & -1.354784 \\
\hline 6 & 4.785161 & -0.167424 & 1.004673 \\
\hline 6 & 5.277027 & -0.481828 & -0.261608 \\
\hline 1 & 5.093538 & -0.043173 & -2.356195 \\
\hline 1 & 5.178897 & -0.710777 & 1.861637 \\
\hline 6 & -3.721101 & -0.165638 & -0.000840 \\
\hline 6 & -3.860421 & -0.550841 & 1.349635 \\
\hline 6 & -3.972693 & -1.097803 & -1.025783 \\
\hline 6 & -4.263862 & -1.851639 & 1.640024 \\
\hline 6 & -4.409387 & -2.381771 & -0.680625 \\
\hline 6 & -4.552322 & -2.784222 & 0.643076 \\
\hline 1 & -4.365981 & -2.152453 & 2.682278 \\
\hline 1 & -4.616972 & -3.087953 & -1.479845 \\
\hline 6 & -5.037233 & -4.168346 & 1.039179 \\
\hline 1 & -4.407043 & -4.498605 & 1.877974 \\
\hline 6 & -3.634304 & 0.410545 & 2.507715 \\
\hline 1 & -3.278331 & 1.363130 & 2.106609 \\
\hline 6 & 3.233021 & 1.870233 & -2.450587 \\
\hline 1 & 2.428878 & 2.549661 & -2.153719 \\
\hline 6 & 3.303268 & 1.063580 & 2.619871 \\
\hline 1 & 2.330847 & 1.559194 & 2.541761 \\
\hline 6 & 6.400316 & -1.494244 & -0.394785 \\
\hline 1 & 6.262734 & -2.233902 & 0.405515 \\
\hline 6 & -3.781220 & -0.783324 & -2.504134 \\
\hline 1 & -3.297510 & 0.192949 & -2.588086 \\
\hline 6 & -5.133215 & -0.711244 & -3.224820 \\
\hline 1 & -5.791364 & 0.037824 & -2.774895 \\
\hline 1 & -4.993493 & -0.457474 & -4.280012 \\
\hline
\end{tabular}




\begin{tabular}{|c|c|c|c|}
\hline 1 & -5.649143 & -1.676359 & -3.175608 \\
\hline 6 & -2.858923 & -1.797638 & -3.191459 \\
\hline 1 & -3.286799 & -2.806494 & -3.181330 \\
\hline 1 & -2.711648 & -1.515903 & -4.238709 \\
\hline 1 & -1.882981 & -1.819496 & -2.701203 \\
\hline 6 & -6.483969 & -4.093963 & 1.547667 \\
\hline 1 & -6.831165 & -5.075778 & 1.883847 \\
\hline 1 & -6.577033 & -3.391004 & 2.379571 \\
\hline 1 & -7.144514 & -3.751848 & 0.744289 \\
\hline 6 & -4.926342 & -5.207049 & -0.076257 \\
\hline 1 & -5.632709 & -4.990857 & -0.884610 \\
\hline 1 & -3.921075 & -5.239009 & -0.509344 \\
\hline 1 & -5.164423 & -6.202230 & 0.309163 \\
\hline 6 & 6.396996 & -2.241609 & -1.727897 \\
\hline 1 & 7.127089 & -3.056459 & -1.707749 \\
\hline 1 & 5.410545 & -2.662770 & -1.942440 \\
\hline 1 & 6.671065 & -1.579542 & -2.555789 \\
\hline 6 & 7.753030 & -0.804973 & -0.169254 \\
\hline 1 & 7.916534 & -0.040915 & -0.936513 \\
\hline 1 & 7.786883 & -0.312855 & 0.806541 \\
\hline 1 & 8.574045 & -1.527056 & -0.223218 \\
\hline 6 & -2.568293 & -0.105950 & 3.479967 \\
\hline 1 & -1.616053 & -0.262840 & 2.967073 \\
\hline 1 & -2.409682 & 0.620106 & 4.283259 \\
\hline 1 & -2.878013 & -1.049113 & 3.943872 \\
\hline 6 & -4.956763 & 0.687624 & 3.234549 \\
\hline 1 & -5.708607 & 1.081293 & 2.544629 \\
\hline 1 & -5.357729 & -0.227709 & 3.682620 \\
\hline 1 & -4.807370 & 1.417368 & 4.036031 \\
\hline 6 & 4.265332 & 1.983900 & 3.383190 \\
\hline 1 & 5.254177 & 1.517652 & 3.455665 \\
\hline 1 & 4.388249 & 2.950980 & 2.890258 \\
\hline 1 & 3.899617 & 2.163520 & 4.399213 \\
\hline 6 & 3.094628 & -0.227566 & 3.419800 \\
\hline 1 & 2.516798 & -0.955329 & 2.848759 \\
\hline 1 & 4.048909 & -0.682228 & 3.706199 \\
\hline 1 & 2.553878 & -0.002685 & 4.344044 \\
\hline 6 & 4.327514 & 2.718700 & -3.108777 \\
\hline 1 & 4.725830 & 3.456336 & -2.406142 \\
\hline 1 & 5.160189 & 2.092507 & -3.446254 \\
\hline 1 & 3.931158 & 3.249462 & -3.979975 \\
\hline 6 & 2.637717 & 0.862651 & -3.440071 \\
\hline 1 & 1.850233 & 0.274052 & -2.962630 \\
\hline 1 & 2.206652 & 1.384812 & -4.299861 \\
\hline 1 & 3.403853 & 0.177046 & -3.817549 \\
\hline 1 & -1.998744 & -3.274327 & -0.607459 \\
\hline 1 & -2.084495 & -5.041265 & 1.023618 \\
\hline 1 & -0.697131 & -5.888913 & 0.306413 \\
\hline 8 & 0.044409 & -3.371548 & -0.844930 \\
\hline 1 & 1.324983 & -1.808897 & 0.993173 \\
\hline 1 & -1.408555 & -4.622983 & -1.612658 \\
\hline 6 & -0.812766 & -2.934515 & 2.108824 \\
\hline 1 & -1.603026 & -3.282227 & 2.780836 \\
\hline 1 & -0.107396 & -2.333160 & 2.686449 \\
\hline 1 & -1.287916 & -2.279581 & 1.368729 \\
\hline
\end{tabular}




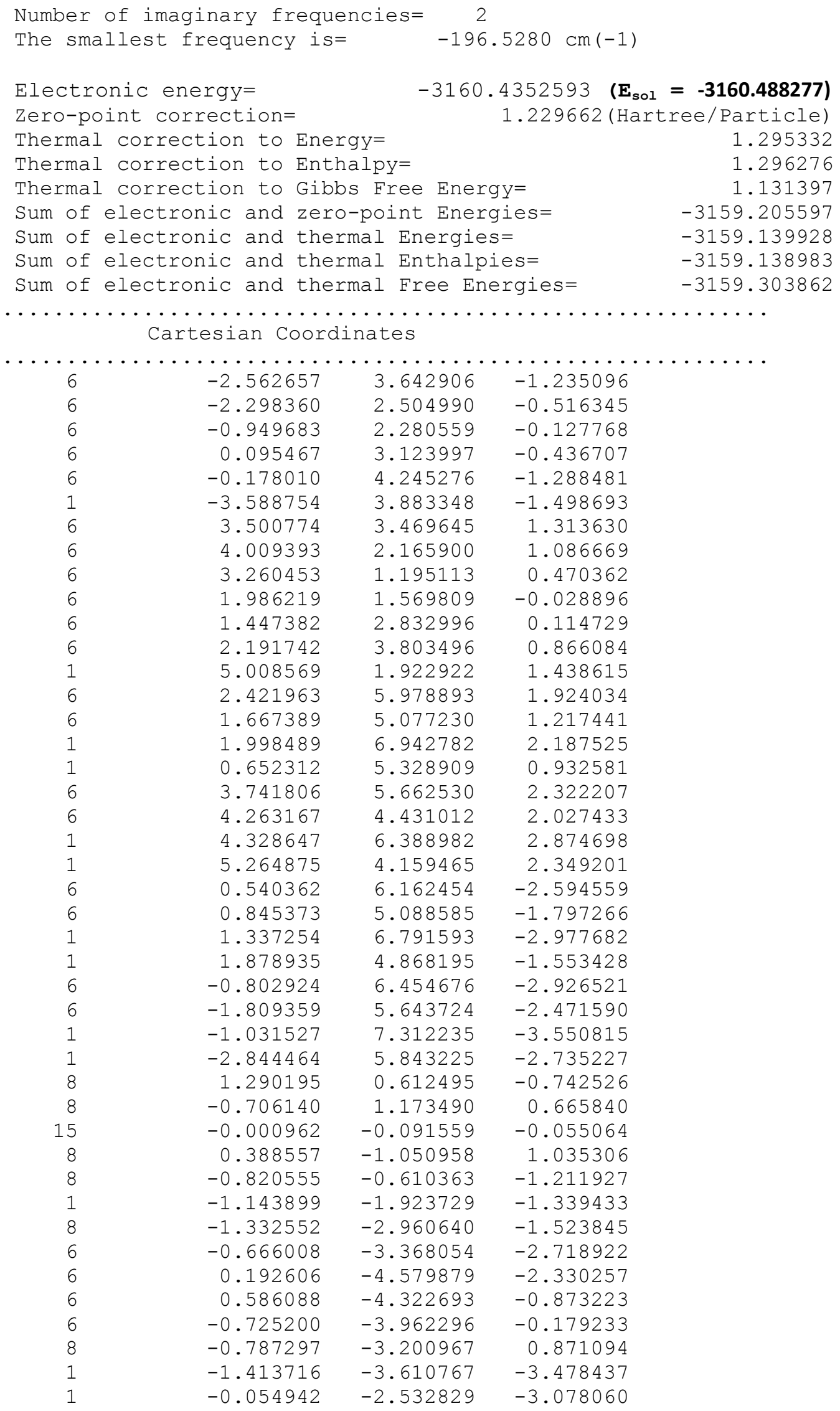




\begin{tabular}{|c|c|c|c|}
\hline 6 & -1.525092 & 4.514829 & -1.659389 \\
\hline 8 & -1.567916 & -4.992225 & -0.204482 \\
\hline 6 & -2.894647 & -4.936915 & 0.416148 \\
\hline 6 & -2.763637 & -5.072775 & 1.930878 \\
\hline 1 & -2.132567 & -5.931276 & 2.176880 \\
\hline 1 & -2.333502 & -4.175976 & 2.375421 \\
\hline 1 & -3.756136 & -5.241036 & 2.360258 \\
\hline 6 & -3.571120 & -6.170992 & -0.171104 \\
\hline 1 & -4.583557 & -6.267298 & 0.228805 \\
\hline 1 & -3.628620 & -6.089519 & -1.259656 \\
\hline & -3.006293 & -7.071558 & 0.083635 \\
\hline 6 & -3.659525 & -3.674907 & 0.02411 \\
\hline 1 & -3.187668 & -2.768649 & 0.411310 \\
\hline 1 & -3.743564 & -3.587877 & -1.061529 \\
\hline 1 & -4.666231 & -3.747243 & 0.448433 \\
\hline 1 & -0.198617 & -2.325079 & 0.909110 \\
\hline 1 & 0.934363 & -5.246263 & -0.39842 \\
\hline 6 & 1.647366 & -3.233676 & -0.756215 \\
\hline 1 & 1.928203 & -3.018352 & 0.278022 \\
\hline 1 & 2.547100 & -3.545786 & -1.291247 \\
\hline 1 & 1.311060 & -2.294816 & -1.207232 \\
\hline 6 & -3.355287 & 1.532236 & -0.097681 \\
\hline 6 & -3.748947 & 1.479811 & 1.252253 \\
\hline 6 & -3.918336 & 0.644181 & -1.035820 \\
\hline 6 & -4.725969 & 0.562784 & 1.638038 \\
\hline 6 & -4.890164 & -0.258274 & -0.59921 \\
\hline 6 & -5.306867 & -0.317657 & 0.72893 \\
\hline 1 & -5.030769 & 0.531361 & 2.68174 \\
\hline 1 & -5.327011 & -0.955418 & -1.311053 \\
\hline 6 & 3.772867 & -0.205625 & 0.354243 \\
\hline 6 & 4.265372 & -0.687133 & -0.87248 \\
\hline 6 & 3.827598 & -1.013436 & 1.50649 \\
\hline 6 & 4.883236 & -1.937568 & -0.906733 \\
\hline 6 & 4.468496 & -2.252189 & 1.424474 \\
\hline 6 & 5.020185 & -2.723747 & 0.236722 \\
\hline 1 & 5.276417 & -2.302193 & -1.854482 \\
\hline 1 & 4.539724 & -2.871913 & 2.315798 \\
\hline 6 & 5.728169 & -4.065031 & 0.186611 \\
\hline 1 & 5.799733 & -4.432214 & 1.21814 \\
\hline 6 & 4.152161 & 0.112696 & -2.16077 \\
\hline 1 & 3.705935 & 1.083759 & -1.92561 \\
\hline 6 & -3.150037 & 2.392241 & 2.31184 \\
\hline 1 & -2.369398 & 3.000403 & 1.845970 \\
\hline 6 & -3.494793 & 0.640237 & -2.49804 \\
\hline 1 & -2.477390 & 1.040697 & -2.54675 \\
\hline 6 & -6.387288 & -1.293846 & 1.15347 \\
\hline 1 & -6.465905 & -2.051444 & 0.362079 \\
\hline 6 & 3.217912 & -0.578689 & 2.832259 \\
\hline 1 & 2.529718 & 0.247848 & 2.62841 \\
\hline 6 & 4.298249 & -0.084824 & 3.80257 \\
\hline 1 & 4.840525 & 0.775086 & 3.40067 \\
\hline 1 & 3.850880 & 0.210449 & 4.756728 \\
\hline 1 & 5.025692 & -0.879919 & 4.000089 \\
\hline 6 & 2.396711 & -1.696769 & 3.48436 \\
\hline 1 & 3.033591 & -2.516820 & 3.83241 \\
\hline 1 & 1.868082 & -1.302406 & 4.35738 \\
\hline 1 & 1.655026 & -2.090240 & 2.787033 \\
\hline 6 & 4.924047 & -5.090007 & -0.62084 \\
\hline
\end{tabular}




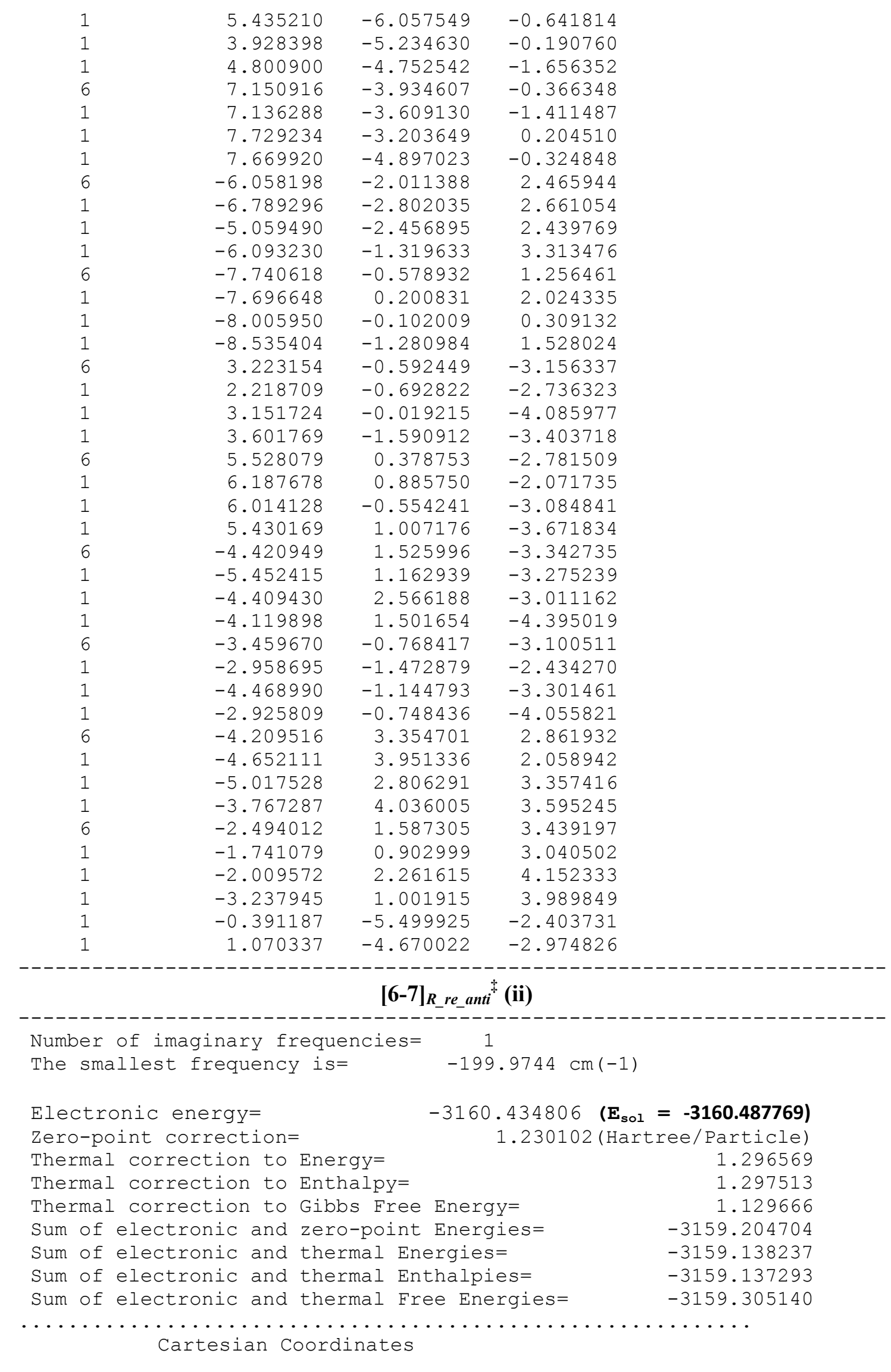




\begin{tabular}{|c|c|c|c|}
\hline 6 & 2.551529 & 3.617442 & 0.971698 \\
\hline 6 & 2.247027 & 2.437476 & 0.339132 \\
\hline 6 & 0.896032 & 2.235320 & -0.047371 \\
\hline 6 & -0.118602 & 3.137286 & 0.203500 \\
\hline 6 & 0.195189 & 4.311442 & 0.964639 \\
\hline 1 & 3.585178 & 3.827229 & 1.233616 \\
\hline 6 & -3.607658 & 3.385121 & -1.407765 \\
\hline 6 & -4.126113 & 2.124364 & -1.015488 \\
\hline 6 & -3.361460 & 1.208454 & -0.336957 \\
\hline 6 & -2.051635 & 1.607534 & 0.036393 \\
\hline 6 & -1.498590 & 2.833013 & -0.266837 \\
\hline 6 & -2.271956 & 3.739726 & -1.064617 \\
\hline 1 & -5.145348 & 1.867062 & -1.291755 \\
\hline 6 & -2.530937 & 5.810663 & -2.307448 \\
\hline 6 & -1.752154 & 4.967607 & -1.556368 \\
\hline 1 & -2.113453 & 6.741341 & -2.677913 \\
\hline 1 & -0.723232 & 5.230146 & -1.336263 \\
\hline 6 & -3.870845 & 5.475035 & -2.612553 \\
\hline 6 & -4.391441 & 4.285831 & -2.175858 \\
\hline 1 & -4.476573 & 6.153374 & -3.204428 \\
\hline 1 & -5.411503 & 4.003303 & -2.421306 \\
\hline 6 & -0.452395 & 6.343896 & 2.126652 \\
\hline 6 & -0.797321 & 5.224668 & 1.412875 \\
\hline 1 & -1.226293 & 7.025384 & 2.464860 \\
\hline 1 & -1.839410 & 5.022484 & 1.191957 \\
\hline 6 & 0.901277 & 6.614600 & 2.435016 \\
\hline 6 & 1.877297 & 5.737140 & 2.042849 \\
\hline 1 & 1.160020 & 7.507081 & 2.995207 \\
\hline 1 & 2.919358 & 5.917720 & 2.292456 \\
\hline 8 & -1.318363 & 0.710574 & 0.790609 \\
\hline 8 & 0.612682 & 1.081890 & -0.758318 \\
\hline 15 & -0.143509 & -0.109737 & 0.040101 \\
\hline 8 & -0.682039 & -1.060801 & -0.991380 \\
\hline 8 & 0.716135 & -0.667486 & 1.149940 \\
\hline 1 & 1.077222 & -1.957264 & 1.104218 \\
\hline 8 & 1.300233 & -3.010697 & 1.174462 \\
\hline 6 & 0.661751 & -3.555125 & 2.329984 \\
\hline 6 & 0.035870 & -4.871845 & 1.865709 \\
\hline 1 & -0.803377 & -5.165249 & 2.500663 \\
\hline 1 & 0.783420 & -5.668589 & 1.881948 \\
\hline 6 & -0.403348 & -4.605304 & 0.424631 \\
\hline 6 & 0.805858 & -3.951637 & -0.240307 \\
\hline 8 & 0.680858 & -3.094503 & -1.213967 \\
\hline 1 & 1.407243 & -3.698656 & 3.116353 \\
\hline 1 & -0.093400 & -2.844377 & 2.683379 \\
\hline 6 & 1.550921 & 4.561560 & 1.316709 \\
\hline 8 & 1.806572 & -4.820280 & -0.359047 \\
\hline 6 & 3.053997 & -4.472272 & -1.048015 \\
\hline 6 & 2.837237 & -4.584811 & -2.554481 \\
\hline 1 & 2.390792 & -5.553698 & -2.795391 \\
\hline 1 & 2.186382 & -3.791045 & -2.919914 \\
\hline 1 & 3.803000 & -4.514317 & -3.063612 \\
\hline 6 & 4.006754 & -5.558920 & -0.561905 \\
\hline 1 & 4.986781 & -5.431011 & -1.028891 \\
\hline 1 & 4.124895 & -5.499849 & 0.523343 \\
\hline 1 & 3.621016 & -6.548945 & -0.819269 \\
\hline 6 & 3.579732 & -3.096524 & -0.646895 \\
\hline
\end{tabular}




\begin{tabular}{|c|c|c|c|}
\hline & 2.918111 & -2.284967 & -0.955588 \\
\hline 1 & 3.728359 & -3.032030 & 0.433995 \\
\hline & 4.545375 & -2.947653 & -1.140432 \\
\hline & 0.009650 & -2.297953 & -1.089348 \\
\hline & -0.555338 & -5.549965 & -0.107435 \\
\hline & -1.665481 & -3.753241 & 0.331746 \\
\hline & -1.949954 & -3.558165 & -0.705698 \\
\hline & -2.494200 & -4.276599 & 0.816974 \\
\hline & -1.561320 & -2.778074 & 0.820214 \\
\hline 6 & 3.307492 & 1.419904 & 0.05665 \\
\hline 6 & 3.795158 & 1.253880 & -1.253507 \\
\hline 6 & 3.854633 & 0.667753 & 1.112422 \\
\hline 6 & 4.878902 & 0.403855 & -1.46393 \\
\hline 6 & 4.952581 & -0.158694 & 0.854270 \\
\hline 6 & 5.494646 & -0.288250 & -0.421019 \\
\hline 1 & 5.265318 & 0.278811 & -2.474871 \\
\hline 1 & 5.380118 & -0.723107 & 1.677460 \\
\hline 6 & -3.855535 & -0.167698 & -0.02866 \\
\hline 6 & -4.074095 & -0.565673 & 1.305999 \\
\hline 6 & -4.113723 & -1.066444 & -1.081516 \\
\hline 6 & -4.589621 & -1.835818 & 1.552462 \\
\hline 6 & -4.652059 & -2.321451 & -0.782000 \\
\hline 6 & -4.901431 & -2.725601 & 0.52488 \\
\hline 1 & -4.765114 & -2.145653 & 2.582169 \\
\hline 1 & -4.862359 & -3.002498 & -1.601430 \\
\hline 6 & -5.516718 & -4.070644 & 0.869371 \\
\hline 1 & -4.996260 & -4.439254 & 1.764941 \\
\hline 6 & -3.797456 & 0.345828 & 2.492028 \\
\hline 1 & -3.378755 & 1.285177 & 2.120932 \\
\hline 6 & 3.178723 & 1.965439 & -2.447211 \\
\hline 1 & 2.348916 & 2.584000 & -2.093103 \\
\hline 6 & 3.275558 & 0.705741 & 2.520750 \\
\hline 1 & 2.249887 & 1.080465 & 2.446626 \\
\hline 6 & 6.732497 & -1.120543 & -0.714428 \\
\hline 1 & 6.536253 & -1.671469 & -1.645559 \\
\hline 6 & -3.825320 & -0.737035 & -2.540494 \\
\hline 1 & -3.239597 & 0.185702 & -2.572595 \\
\hline 6 & -5.127225 & -0.509443 & -3.318774 \\
\hline 1 & -5.727775 & 0.292115 & -2.879590 \\
\hline 1 & -4.912196 & -0.246439 & -4.359045 \\
\hline 1 & -5.739139 & -1.418199 & -3.320392 \\
\hline 6 & -2.983423 & -1.824346 & -3.218426 \\
\hline 1 & -3.520977 & -2.776962 & -3.27474 \\
\hline 1 & -2.745207 & -1.523558 & -4.243489 \\
\hline 1 & -2.047197 & -1.973806 & -2.677463 \\
\hline 6 & -6.997527 & -3.891573 & 1.232096 \\
\hline 1 & -7.443394 & -4.842925 & 1.538934 \\
\hline 1 & -7.122106 & -3.171862 & 2.045559 \\
\hline 1 & -7.550829 & -3.516242 & 0.364873 \\
\hline 6 & -5.364616 & -5.121935 & -0.229547 \\
\hline 1 & -5.967535 & -4.863902 & -1.106354 \\
\hline 1 & -4.324208 & -5.222407 & -0.553678 \\
\hline 1 & -5.710655 & -6.095205 & 0.129052 \\
\hline 6 & 7.932019 & -0.196006 & -0.967328 \\
\hline 1 & 8.821529 & -0.774681 & -1.235170 \\
\hline 1 & 7.724794 & 0.512973 & -1.773211 \\
\hline 1 & 8.156326 & 0.379983 & -0.063566 \\
\hline 6 & 7.075934 & -2.136910 & 0.37353 \\
\hline
\end{tabular}




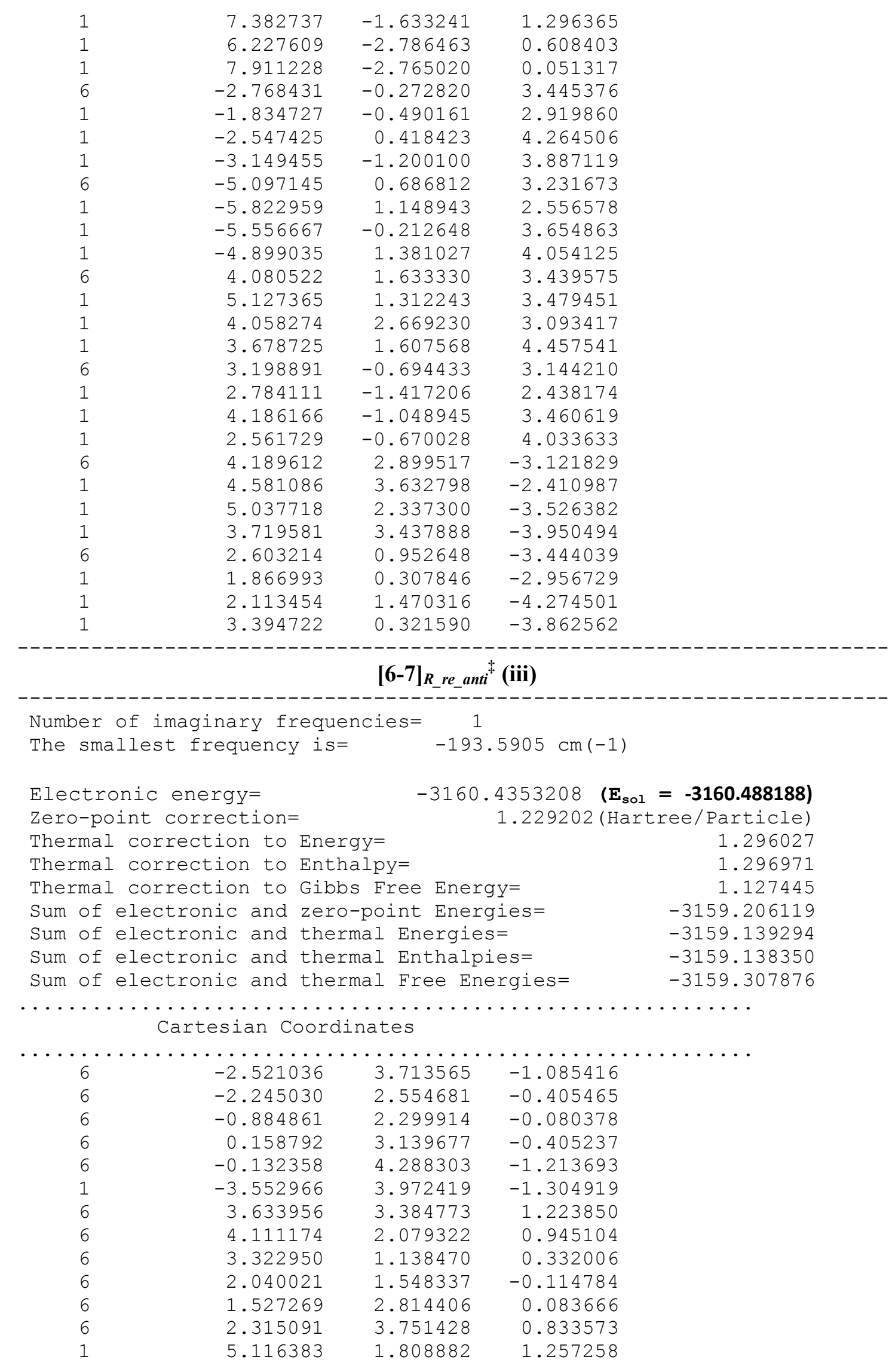




\begin{tabular}{|c|c|c|c|}
\hline 6 & 2.619043 & 5.892752 & 1.941500 \\
\hline 6 & 1.825135 & 5.023008 & 1.237925 \\
\hline 1 & 2.221626 & 6.855396 & 2.246658 \\
\hline 1 & 0.805367 & 5.298284 & 0.994207 \\
\hline 6 & 3.946409 & 5.544148 & 2.284064 \\
\hline 6 & 4.436482 & 4.313425 & 1.936769 \\
\hline 1 & 4.564276 & 6.244697 & 2.836180 \\
\hline 1 & 5.444032 & 4.017582 & 2.215995 \\
\hline 6 & 0.560810 & 6.231540 & -2.494839 \\
\hline 6 & 0.881738 & 5.134145 & -1.736854 \\
\hline 1 & 1.350661 & 6.862192 & -2.889859 \\
\hline 1 & 1.920746 & 4.897741 & -1.534829 \\
\hline 6 & -0.789910 & 6.545715 & -2.772021 \\
\hline 6 & -1.788782 & 5.734587 & -2.301164 \\
\hline 1 & -1.030599 & 7.420606 & -3.366989 \\
\hline 1 & -2.830219 & 5.951315 & -2.522923 \\
\hline 8 & 1.307198 & 0.621983 & -0.831993 \\
\hline 8 & -0.625455 & 1.163759 & 0.666128 \\
\hline 15 & 0.027400 & -0.086121 & -0.129132 \\
\hline 8 & 0.433889 & -1.097267 & 0.907256 \\
\hline 8 & -0.841023 & -0.543258 & -1.275919 \\
\hline 1 & -1.191020 & -1.844066 & -1.445055 \\
\hline 8 & -1.408106 & -2.869724 & -1.659760 \\
\hline 6 & -0.762990 & -3.261202 & -2.871220 \\
\hline 6 & 0.032517 & -4.528080 & -2.530026 \\
\hline 1 & 0.896112 & -4.646740 & -3.188829 \\
\hline 1 & -0.602323 & -5.412018 & -2.622516 \\
\hline 6 & 0.455100 & -4.337036 & -1.071156 \\
\hline 6 & -0.826817 & -3.931157 & -0.348120 \\
\hline 8 & -0.836956 & -3.191933 & 0.719505 \\
\hline 1 & -1.521168 & -3.436485 & -3.638695 \\
\hline 1 & -0.109921 & -2.444990 & -3.198973 \\
\hline 6 & -1.488861 & 4.582782 & -1.527619 \\
\hline 8 & -1.715082 & -4.921533 & -0.382003 \\
\hline 6 & -3.027784 & -4.822811 & 0.262671 \\
\hline 6 & -2.879371 & -4.978449 & 1.774038 \\
\hline 1 & -2.270133 & -5.857352 & 2.002664 \\
\hline 1 & -2.417238 & -4.099618 & 2.222500 \\
\hline 1 & -3.870570 & -5.123138 & 2.215011 \\
\hline 6 & -3.759877 & -6.024590 & -0.324732 \\
\hline 1 & -4.769396 & -6.085754 & 0.089307 \\
\hline 1 & -3.830309 & -5.931136 & -1.411526 \\
\hline 1 & -3.226436 & -6.948466 & -0.086181 \\
\hline 6 & -3.748402 & -3.528780 & -0.106629 \\
\hline 1 & -3.237151 & -2.643414 & 0.279646 \\
\hline 1 & -3.845562 & -3.429257 & -1.190206 \\
\hline 1 & -4.750685 & -3.565244 & 0.332462 \\
\hline 1 & -0.209196 & -2.344074 & 0.765715 \\
\hline 1 & 0.759007 & -5.291909 & -0.628779 \\
\hline 6 & 1.575208 & -3.311594 & -0.932290 \\
\hline 1 & 1.874181 & -3.142963 & 0.105635 \\
\hline 1 & 2.453565 & -3.657737 & -1.482828 \\
\hline 1 & 1.291658 & -2.341241 & -1.352923 \\
\hline 6 & -3.300025 & 1.588020 & 0.030499 \\
\hline 6 & -3.619192 & 1.483399 & 1.397498 \\
\hline 6 & -3.942207 & 0.760811 & -0.911726 \\
\hline 6 & -4.600443 & 0.576879 & 1.796413 \\
\hline 6 & -4.914738 & -0.134741 & -0.461297 \\
\hline
\end{tabular}




\begin{tabular}{|c|c|c|c|}
\hline 6 & -5.258237 & -0.243844 & 0.883919 \\
\hline 1 & -4.849746 & 0.507473 & 2.852929 \\
\hline 1 & -5.411900 & -0.785687 & -1.177660 \\
\hline 6 & 3.791489 & -0.273313 & 0.176994 \\
\hline 6 & 4.227791 & -0.751054 & -1.068673 \\
\hline 6 & 3.844685 & -1.104243 & 1.316312 \\
\hline 6 & 4.784813 & -2.031026 & -1.142023 \\
\hline 6 & 4.422482 & -2.367277 & 1.195524 \\
\hline 6 & 4.908559 & -2.845937 & -0.021663 \\
\hline & 5.135502 & -2.403616 & -2.103245 \\
\hline & 4.485698 & -3.000970 & 2.077399 \\
\hline 6 & 5.547320 & -4.218595 & -0.118324 \\
\hline 1 & 5.768868 & -4.402274 & -1.177623 \\
\hline 6 & 4.113682 & 0.076203 & -2.338988 \\
\hline 1 & 3.707718 & 1.057620 & -2.074684 \\
\hline 6 & -2.944085 & 2.335641 & 2.461454 \\
\hline 1 & -2.157774 & 2.928368 & 1.98593 \\
\hline 6 & -3.610544 & 0.815795 & -2.395873 \\
\hline 1 & -2.606198 & 1.238374 & -2.494238 \\
\hline 6 & -6.342166 & -1.207035 & 1.328624 \\
\hline 1 & -6.501226 & -1.915844 & 0.504809 \\
\hline 6 & 3.288707 & -0.665558 & 2.66463 \\
\hline 1 & 2.615191 & 0.179497 & 2.488064 \\
\hline 6 & 4.411084 & -0.207131 & 3.60411 \\
\hline 1 & 4.965176 & 0.639804 & 3.191252 \\
\hline 1 & 4.000981 & 0.094406 & 4.572934 \\
\hline 1 & 5.121690 & -1.023354 & 3.775294 \\
\hline 6 & 2.459021 & -1.765995 & 3.335631 \\
\hline 1 & 3.083645 & -2.604926 & 3.660278 \\
\hline 1 & 1.968542 & -1.363141 & 4.226860 \\
\hline 1 & 1.686591 & -2.135058 & 2.658617 \\
\hline 6 & 6.869336 & -4.260482 & 0.656551 \\
\hline 1 & 7.350356 & -5.238051 & 0.553269 \\
\hline 1 & 7.559945 & -3.493410 & 0.296663 \\
\hline 1 & 6.693858 & -4.080724 & 1.722355 \\
\hline 6 & 4.603769 & -5.325083 & 0.365702 \\
\hline 1 & 4.365663 & -5.198855 & 1.426670 \\
\hline 1 & 3.662125 & -5.316677 & -0.190683 \\
\hline 1 & 5.067794 & -6.308408 & 0.242781 \\
\hline 6 & -5.948949 & -2.006611 & 2.574723 \\
\hline 1 & -6.690985 & -2.785014 & 2.777981 \\
\hline 1 & -4.969759 & -2.479199 & 2.454786 \\
\hline 1 & -5.902452 & -1.362243 & 3.458337 \\
\hline 6 & -7.659965 & -0.458057 & 1.563909 \\
\hline 1 & -7.538108 & 0.278405 & 2.36531 \\
\hline 1 & -7.974088 & 0.076095 & 0.663211 \\
\hline 1 & -8.456978 & -1.149712 & 1.854853 \\
\hline 6 & 3.137769 & -0.576329 & -3.325660 \\
\hline 1 & 2.140812 & -0.653673 & -2.88322 \\
\hline 1 & 3.062735 & 0.019406 & -4.240769 \\
\hline 1 & 3.477364 & -1.580463 & -3.603930 \\
\hline 6 & 5.484190 & 0.304074 & -2.986391 \\
\hline 1 & 6.178500 & 0.769913 & -2.281588 \\
\hline 1 & 5.926562 & -0.640097 & -3.32046 \\
\hline 1 & 5.390440 & 0.955180 & -3.86070 \\
\hline 6 & -4.602048 & 1.715637 & -3.14595 \\
\hline 1 & -5.620335 & 1.326923 & -3.035438 \\
\hline 1 & -4.593331 & 2.740382 & -2.76815 \\
\hline
\end{tabular}




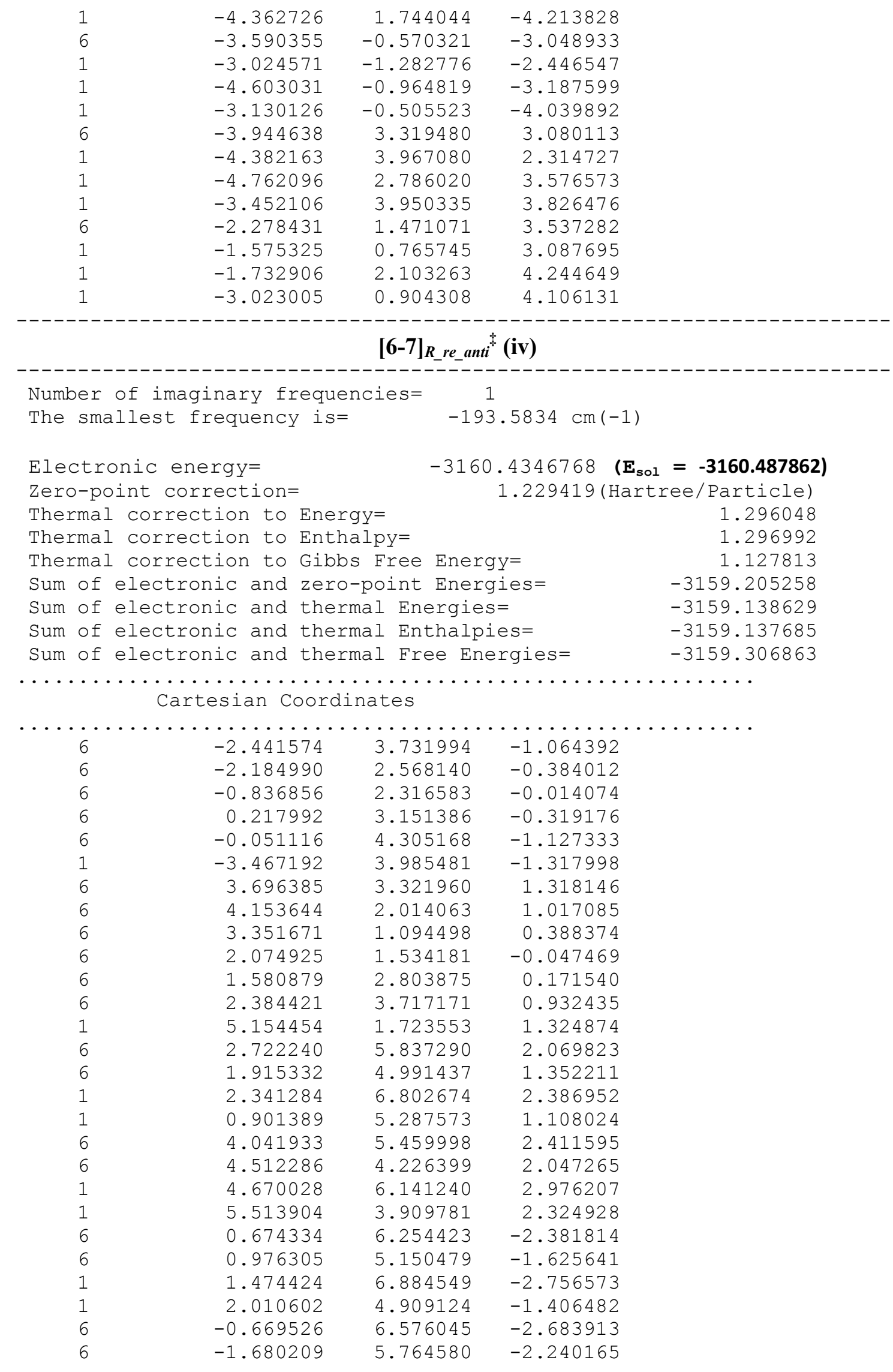




\begin{tabular}{|c|c|c|c|}
\hline 1 & -0.895030 & 7.456182 & -3.277094 \\
\hline 1 & -2.716292 & 5.985452 & -2.482141 \\
\hline 8 & 1.315804 & 0.629235 & -0.766691 \\
\hline 8 & -0.600156 & 1.185362 & 0.746242 \\
\hline 15 & 0.070316 & -0.078896 & -0.009557 \\
\hline 8 & 0.519930 & -1.033762 & 1.059710 \\
\hline 8 & -0.810362 & -0.601742 & -1.120545 \\
\hline 1 & -1.263075 & -1.866282 & -1.113238 \\
\hline 8 & -1.553023 & -2.899077 & -1.229879 \\
\hline 6 & -1.083247 & -3.372697 & -2.496629 \\
\hline 6 & -0.237053 & -4.618677 & -2.199255 \\
\hline 1 & 0.545830 & -4.756554 & -2.948613 \\
\hline 1 & -0.866110 & -5.511011 & -2.181887 \\
\hline 6 & 0.345347 & -4.361262 & -0.808116 \\
\hline 6 & -0.854704 & -3.909298 & 0.022032 \\
\hline 8 & -0.730778 & -3.148087 & 1.072578 \\
\hline 1 & -1.943178 & -3.591379 & -3.134305 \\
\hline 1 & -0.491224 & -2.575643 & -2.959504 \\
\hline 6 & -1.399573 & 4.606408 & -1.468722 \\
\hline 8 & -1.753221 & -4.888978 & 0.123238 \\
\hline 6 & -2.964225 & -4.750385 & 0.937653 \\
\hline 6 & -2.607933 & -4.978458 & 2.404117 \\
\hline 1 & -2.048120 & -5.911917 & 2.509719 \\
\hline 1 & -2.009170 & -4.157118 & 2.796912 \\
\hline 1 & -3.527816 & -5.060601 & 2.990650 \\
\hline 6 & -3.835119 & -5.888205 & 0.416220 \\
\hline 1 & -4.775144 & -5.923787 & 0.972581 \\
\hline 1 & -4.059208 & -5.737899 & -0.643171 \\
\hline 1 & -3.321681 & -6.846120 & 0.533327 \\
\hline 6 & -3.664640 & -3.409722 & 0.721867 \\
\hline 1 & -3.073385 & -2.568405 & 1.086668 \\
\hline 1 & -3.882345 & -3.246570 & -0.336192 \\
\hline 1 & -4.609771 & -3.430074 & 1.274508 \\
\hline 1 & -0.114230 & -2.299619 & 1.017753 \\
\hline 1 & 0.695562 & -5.294182 & -0.354081 \\
\hline 6 & 1.467999 & -3.328660 & -0.837357 \\
\hline 1 & 1.872408 & -3.112269 & 0.155445 \\
\hline 1 & 2.287219 & -3.694294 & -1.461122 \\
\hline 1 & 1.128458 & -2.382376 & -1.269597 \\
\hline 6 & -3.265510 & 1.594989 & -0.033932 \\
\hline 6 & -3.664453 & 1.434421 & 1.307841 \\
\hline 6 & -3.885511 & 0.839512 & -1.046190 \\
\hline 6 & -4.714921 & 0.567713 & 1.597567 \\
\hline 6 & -4.948161 & -0.004194 & -0.706345 \\
\hline 6 & -5.388807 & -0.145656 & 0.605595 \\
\hline 1 & -5.028070 & 0.446490 & 2.633639 \\
\hline 1 & -5.430002 & -0.572072 & -1.496286 \\
\hline 6 & 3.799259 & -0.321656 & 0.208406 \\
\hline 6 & 4.163871 & -0.804608 & -1.062014 \\
\hline 6 & 3.913583 & -1.152945 & 1.340189 \\
\hline 6 & 4.718029 & -2.081174 & -1.165787 \\
\hline 6 & 4.490856 & -2.416258 & 1.186729 \\
\hline 6 & 4.918172 & -2.891579 & -0.049400 \\
\hline 1 & 5.015499 & -2.444211 & -2.148396 \\
\hline 1 & 4.612223 & -3.052647 & 2.060809 \\
\hline 6 & 5.574327 & -4.254210 & -0.175648 \\
\hline 1 & 5.687960 & -4.654711 & 0.839558 \\
\hline 6 & 3.996590 & 0.024375 & -2.325993 \\
\hline
\end{tabular}




\begin{tabular}{|c|c|c|c|}
\hline 1 & 3.581126 & 0.997816 & -2.049462 \\
\hline 6 & -3.005368 & 2.187771 & 2.453217 \\
\hline 1 & -2.180584 & 2.782366 & 2.050673 \\
\hline 6 & -3.427164 & 0.889544 & -2.497560 \\
\hline 1 & -2.410591 & 1.293881 & -2.511062 \\
\hline 6 & -6.580362 & -1.006259 & 0.994354 \\
\hline 1 & -6.272130 & -1.609767 & 1.859982 \\
\hline 6 & 3.431661 & -0.726653 & 2.720473 \\
\hline 1 & 2.780013 & 0.143523 & 2.593914 \\
\hline 6 & 4.608284 & -0.331701 & 3.621929 \\
\hline 1 & 5.178449 & 0.502339 & 3.204639 \\
\hline 1 & 4.249893 & -0.037071 & 4.613058 \\
\hline 1 & 5.293349 & -1.177592 & 3.746562 \\
\hline 6 & 2.596049 & -1.817694 & 3.400441 \\
\hline 1 & 3.204088 & -2.689515 & 3.663363 \\
\hline 1 & 2.169413 & -1.427235 & 4.329370 \\
\hline 1 & 1.775306 & -2.136247 & 2.755584 \\
\hline 6 & 4.698842 & -5.230109 & -0.968923 \\
\hline 1 & 5.180176 & -6.209952 & -1.046291 \\
\hline 1 & 3.723750 & -5.361580 & -0.490658 \\
\hline 1 & 4.530541 & -4.857798 & -1.985919 \\
\hline 6 & 6.970158 & -4.148264 & -0.798885 \\
\hline 1 & 6.911665 & -3.776496 & -1.826978 \\
\hline 1 & 7.603189 & -3.462433 & -0.230053 \\
\hline 1 & 7.456574 & -5.128163 & -0.825860 \\
\hline 6 & -7.746714 & -0.112414 & 1.441115 \\
\hline 1 & -8.597174 & -0.717336 & 1.770790 \\
\hline 1 & -7.454163 & 0.547117 & 2.262160 \\
\hline 1 & -8.075351 & 0.516497 & 0.607211 \\
\hline 6 & -7.047784 & -1.958284 & -0.104859 \\
\hline 1 & -7.452805 & -1.401155 & -0.956526 \\
\hline 1 & -6.234153 & -2.591974 & -0.468722 \\
\hline 1 & -7.844286 & -2.606091 & 0.272045 \\
\hline 6 & 3.007307 & -0.640955 & -3.289783 \\
\hline 1 & 2.022827 & -0.738656 & -2.824227 \\
\hline 1 & 2.899141 & -0.042332 & -4.199670 \\
\hline 1 & 3.356553 & -1.637434 & -3.582754 \\
\hline 6 & 5.346373 & 0.278415 & -3.006975 \\
\hline 1 & 6.047063 & 0.761588 & -2.320258 \\
\hline 1 & 5.800429 & -0.658118 & -3.347074 \\
\hline 1 & 5.217998 & 0.924048 & -3.881059 \\
\hline 6 & -4.330383 & 1.796479 & -3.343335 \\
\hline 1 & -5.365991 & 1.441141 & -3.304151 \\
\hline 1 & -4.317638 & 2.830519 & -2.991636 \\
\hline 1 & -4.008833 & 1.789898 & -4.389738 \\
\hline 6 & -3.372855 & -0.507854 & -3.128600 \\
\hline 1 & -2.898962 & -1.221311 & -2.452172 \\
\hline 1 & -4.375092 & -0.878765 & -3.370260 \\
\hline 1 & -2.803525 & -0.474426 & -4.062610 \\
\hline 6 & -3.995198 & 3.161721 & 3.103896 \\
\hline 1 & -4.388291 & 3.871105 & 2.370004 \\
\hline 1 & -4.844027 & 2.624461 & 3.540127 \\
\hline 1 & -3.507630 & 3.726352 & 3.904585 \\
\hline 6 & -2.415302 & 1.224027 & 3.488519 \\
\hline 1 & -1.708178 & 0.535065 & 3.020058 \\
\hline 1 & -1.888902 & 1.782256 & 4.268755 \\
\hline 1 & -3.202510 & 0.637695 & 3.974502 \\
\hline
\end{tabular}




\section{$[6-7]_{R \_ \text {si anti }}{ }^{*}(\mathbf{i})$}

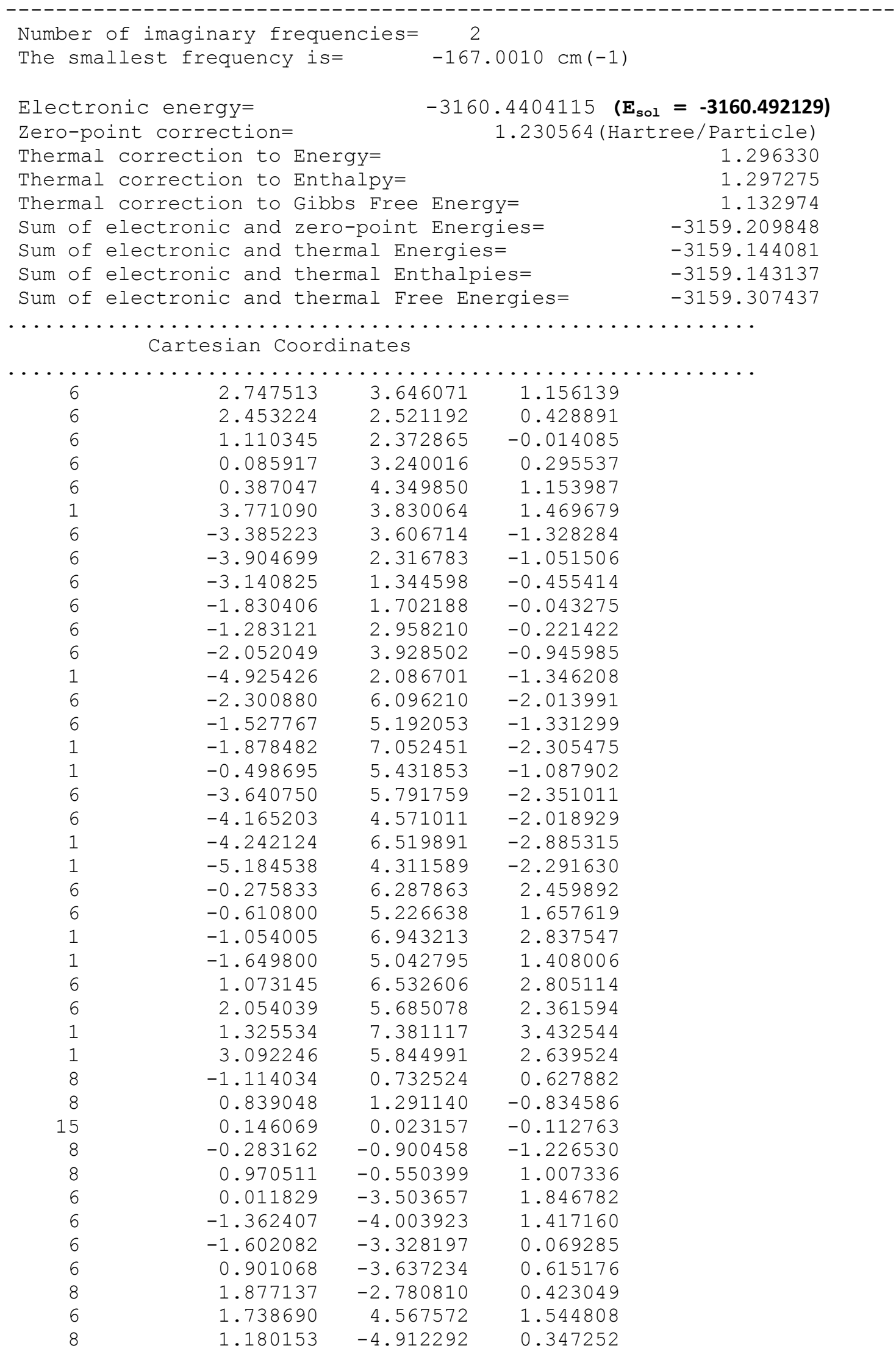




\begin{tabular}{|c|c|c|c|}
\hline 6 & 2.188311 & -5.293679 & -0.641804 \\
\hline 6 & 1.919985 & -6.783474 & -0.822539 \\
\hline 1 & 2.633241 & -7.207572 & -1.533680 \\
\hline 1 & 2.023448 & -7.305606 & 0.132382 \\
\hline$\perp$ & 0.907361 & -6.943780 & -1.201069 \\
\hline 6 & 3.583157 & -5.074027 & -0.058317 \\
\hline 1 & 4.326610 & -5.524005 & -0.723840 \\
\hline 1 & 3.806491 & -4.012968 & 0.055431 \\
\hline 1 & 3.656114 & -5.559423 & 0.919397 \\
\hline 6 & 1.987221 & -4.552634 & -1.960416 \\
\hline 1 & 0.976456 & -4.716246 & -2.340769 \\
\hline 1 & 2.150528 & -3.479424 & -1.84852 \\
\hline 1 & 2.705524 & -4.939681 & -2.689539 \\
\hline 1 & -0.208136 & -2.236929 & -0.918840 \\
\hline 6 & 3.444552 & 1.447964 & 0.103195 \\
\hline 6 & 3.881258 & 1.281929 & -1.224089 \\
\hline 6 & 3.866885 & 0.549870 & 1.105668 \\
\hline 6 & 4.752318 & 0.235622 & -1.526217 \\
\hline 6 & 4.730354 & -0.487638 & 0.749622 \\
\hline 6 & 5.180018 & -0.666698 & -0.556027 \\
\hline 1 & 5.093007 & 0.117101 & -2.552733 \\
\hline 1 & 5.051513 & -1.195877 & 1.509541 \\
\hline 6 & -3.668176 & -0.042506 & -0.262608 \\
\hline 6 & -3.976573 & -0.520888 & 1.027157 \\
\hline 6 & -3.913002 & -0.853124 & -1.388497 \\
\hline 6 & -4.592850 & -1.766322 & 1.158235 \\
\hline 6 & -4.557073 & -2.080688 & -1.206609 \\
\hline 6 & -4.921476 & -2.549630 & 0.051474 \\
\hline 1 & -4.848131 & -2.120235 & 2.155727 \\
\hline 1 & -4.778935 & -2.693935 & -2.077728 \\
\hline 6 & -5.672027 & -3.858491 & 0.211737 \\
\hline 1 & -5.768673 & -4.302700 & -0.787039 \\
\hline 6 & -3.672217 & 0.271512 & 2.288656 \\
\hline 1 & -3.197610 & 1.213899 & 2.002552 \\
\hline 6 & 3.442210 & 2.211068 & -2.345191 \\
\hline 1 & 2.699011 & 2.907954 & -1.946116 \\
\hline 6 & 3.417481 & 0.678130 & 2.554781 \\
\hline 1 & 2.463160 & 1.213678 & 2.561990 \\
\hline 6 & 6.124473 & -1.802886 & -0.899313 \\
\hline 1 & 6.146831 & -2.477134 & -0.033148 \\
\hline 6 & -3.489504 & -0.455499 & -2.795493 \\
\hline 1 & -2.850661 & 0.429124 & -2.720787 \\
\hline 6 & -4.704440 & -0.103156 & -3.661991 \\
\hline 1 & -5.277163 & 0.725476 & -3.236270 \\
\hline 1 & -4.387755 & 0.181705 & -4.669799 \\
\hline 1 & -5.376588 & -0.963836 & -3.749100 \\
\hline 6 & -2.658363 & -1.558482 & -3.462694 \\
\hline 1 & -3.257415 & -2.455486 & -3.652270 \\
\hline 1 & -2.281523 & -1.205852 & -4.427519 \\
\hline 1 & -1.801997 & -1.827076 & -2.840633 \\
\hline 6 & -4.920037 & -4.854617 & 1.099626 \\
\hline 1 & -5.476812 & -5.792867 & 1.183043 \\
\hline 1 & -3.929665 & -5.081795 & 0.694518 \\
\hline 1 & -4.788034 & -4.455414 & 2.11112 \\
\hline 6 & -7.083404 & -3.609881 & 0.757106 \\
\hline 1 & -7.036583 & -3.174152 & 1.760757 \\
\hline 1 & -7.635946 & -2.917621 & 0.116648 \\
\hline 1 & -7.644895 & -4.546865 & 0.82 \\
\hline
\end{tabular}




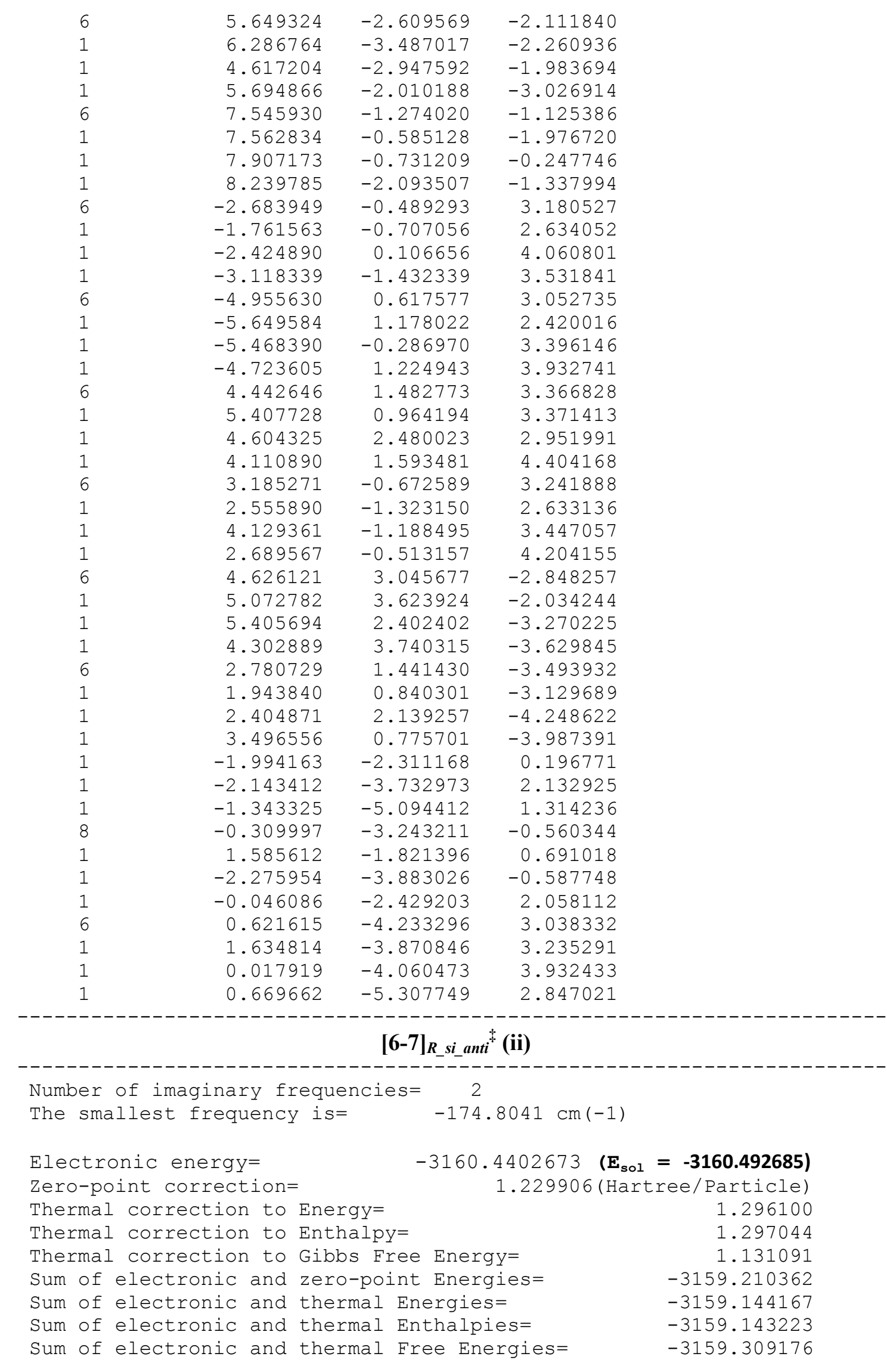




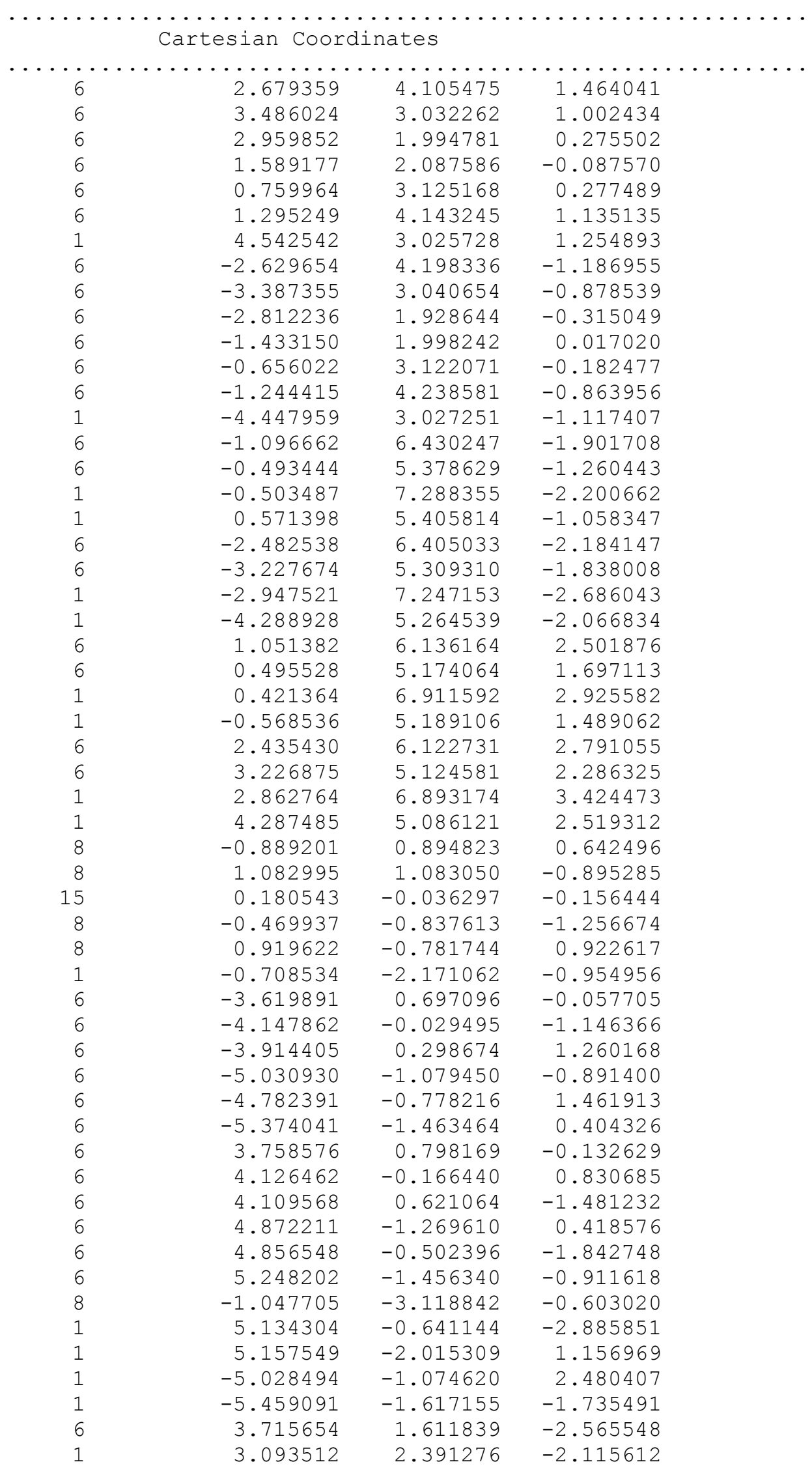




\begin{tabular}{|c|c|c|c|}
\hline 6 & 3.742033 & -0.036198 & 2.298675 \\
\hline 1 & 2.874728 & 0.628369 & 2.358496 \\
\hline 6 & 6.041636 & -2.677583 & -1.334692 \\
\hline 1 & 6.202950 & -2.601001 & -2.417513 \\
\hline 6 & 2.885790 & 0.938369 & -3.664176 \\
\hline 1 & 2.005860 & 0.449264 & -3.239709 \\
\hline 1 & 2.551564 & 1.681122 & -4.395227 \\
\hline 1 & 3.475580 & 0.187128 & -4.200115 \\
\hline 6 & 4.954585 & 2.299831 & -3.150875 \\
\hline 1 & 5.525655 & 2.810141 & -2.369963 \\
\hline 1 & 5.616794 & 1.571713 & -3.630894 \\
\hline 1 & 4.662963 & 3.037574 & -3.904916 \\
\hline 6 & 3.333760 & -1.370884 & 2.932473 \\
\hline 1 & 4.195662 & -2.032276 & 3.070933 \\
\hline 1 & 2.903961 & -1.189759 & 3.922333 \\
\hline 1 & 2.590924 & -1.888716 & 2.324126 \\
\hline 6 & 4.893096 & 0.576072 & 3.108806 \\
\hline 1 & 4.612665 & 0.675505 & 4.162308 \\
\hline 1 & 5.777202 & -0.068449 & 3.051522 \\
\hline 1 & 5.177558 & 1.563302 & 2.737602 \\
\hline 6 & 5.258717 & -3.966811 & -1.063658 \\
\hline 1 & 5.099059 & -4.101973 & 0.011528 \\
\hline 1 & 4.277418 & -3.939646 & -1.546675 \\
\hline 1 & 5.805664 & -4.840342 & -1.432942 \\
\hline 6 & 7.413863 & -2.719828 & -0.654265 \\
\hline 1 & 7.993040 & -3.581279 & -1.001153 \\
\hline 1 & 7.984145 & -1.811121 & -0.863945 \\
\hline 1 & 7.305263 & -2.805204 & 0.432053 \\
\hline 6 & -3.768377 & 0.262361 & -2.591722 \\
\hline 1 & -2.980142 & 1.020178 & -2.591441 \\
\hline 6 & -3.350030 & 1.006949 & 2.481868 \\
\hline 1 & -2.711944 & 1.828333 & 2.145727 \\
\hline 6 & -6.376011 & -2.575161 & 0.655271 \\
\hline 1 & -6.473802 & -2.686021 & 1.742812 \\
\hline 6 & -7.753176 & -2.203801 & 0.093201 \\
\hline 1 & -8.489302 & -2.978093 & 0.329642 \\
\hline 1 & -8.103282 & -1.254028 & 0.505501 \\
\hline 1 & -7.711613 & -2.101617 & -0.996208 \\
\hline 6 & -5.904300 & -3.917004 & 0.086189 \\
\hline 1 & -5.747374 & -3.849162 & -0.995933 \\
\hline 1 & -4.964097 & -4.235287 & 0.546092 \\
\hline 1 & -6.651225 & -4.696096 & 0.266148 \\
\hline 6 & -2.477084 & 0.056426 & 3.309362 \\
\hline 1 & -3.062660 & -0.790525 & 3.684936 \\
\hline 1 & -1.649176 & -0.323914 & 2.704776 \\
\hline 1 & -2.055051 & 0.578780 & 4.173246 \\
\hline 6 & -4.468552 & 1.618014 & 3.334267 \\
\hline 1 & -5.075585 & 2.311627 & 2.745729 \\
\hline 1 & -5.132554 & 0.844155 & 3.732607 \\
\hline 1 & -4.044637 & 2.164039 & 4.182385 \\
\hline 6 & -4.961326 & 0.812873 & -3.38140 \\
\hline 1 & -4.669387 & 1.035134 & -4.412184 \\
\hline 1 & -5.777810 & 0.083153 & -3.413358 \\
\hline 1 & -5.353329 & 1.729907 & -2.932057 \\
\hline 6 & -3.193102 & -0.985092 & -3.27376 \\
\hline 1 & -2.879198 & -0.741580 & -4.293345 \\
\hline 1 & -2.320722 & -1.352620 & -2.72808 \\
\hline 1 & -3.936967 & -1.786608 & -3.33978 \\
\hline
\end{tabular}




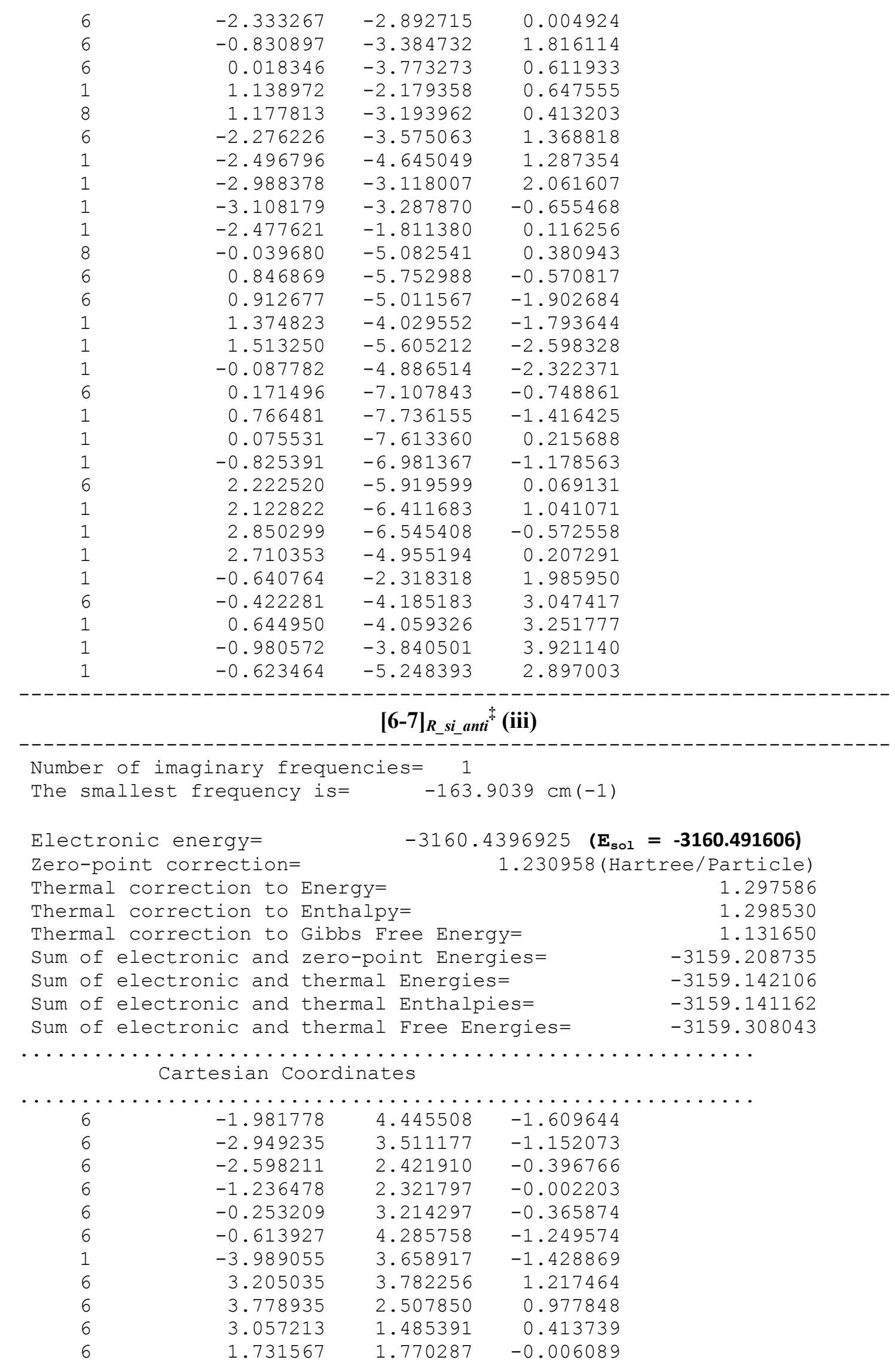




\begin{tabular}{|c|c|c|c|}
\hline 6 & 1.132290 & 3.006684 & 0.138508 \\
\hline 6 & 1.858848 & 4.032099 & 0.830089 \\
\hline 1 & 4.809998 & 2.331307 & 1.273299 \\
\hline 6 & 2.012410 & 6.239940 & 1.831489 \\
\hline 6 & 1.278954 & 5.282131 & 1.179077 \\
\hline 1 & 1.547773 & 7.184666 & 2.094845 \\
\hline 1 & 0.239604 & 5.469208 & 0.934020 \\
\hline 6 & 3.365147 & 6.006657 & 2.172495 \\
\hline 6 & 3.943215 & 4.800948 & 1.875671 \\
\hline 1 & 3.934074 & 6.777259 & 2.682221 \\
\hline 1 & 4.973845 & 4.595325 & 2.152032 \\
\hline 6 & -0.051481 & 6.199963 & -2.634754 \\
\hline 6 & 0.340192 & 5.179765 & -1.805367 \\
\hline 1 & 0.693379 & 6.869463 & -3.052654 \\
\hline 1 & 1.390232 & 5.041570 & -1.572390 \\
\hline 6 & -1.416111 & 6.385014 & -2.954582 \\
\hline 6 & -2.356107 & 5.522037 & -2.455557 \\
\hline 1 & -1.712707 & 7.201673 & -3.604516 \\
\hline 1 & -3.406005 & 5.637565 & -2.710516 \\
\hline 8 & 1.053572 & 0.752788 & -0.647422 \\
\hline 8 & -0.900638 & 1.270095 & 0.835017 \\
\hline 15 & -0.180018 & 0.015490 & 0.115810 \\
\hline 8 & 0.296977 & -0.887468 & 1.226464 \\
\hline 8 & -1.011507 & -0.588554 & -0.984147 \\
\hline 1 & 0.303133 & -2.235127 & 0.922612 \\
\hline 6 & 3.653343 & 0.122047 & 0.257939 \\
\hline 6 & 3.905893 & -0.657347 & 1.404020 \\
\hline 6 & 4.017841 & -0.358556 & -1.014997 \\
\hline 6 & 4.618232 & -1.851265 & 1.260244 \\
\hline 6 & 4.698949 & -1.573271 & -1.109648 \\
\hline 6 & 5.038163 & -2.320298 & 0.018779 \\
\hline 6 & -3.559726 & 1.358288 & 0.029600 \\
\hline 6 & -4.035940 & 0.413495 & -0.900484 \\
\hline 6 & -3.936877 & 1.269726 & 1.383399 \\
\hline 6 & -4.892304 & -0.597268 & -0.453989 \\
\hline 6 & -4.801636 & 0.251944 & 1.775249 \\
\hline 6 & -5.283823 & -0.699303 & 0.877760 \\
\hline 8 & 0.471799 & -3.230707 & 0.567970 \\
\hline 1 & -5.103594 & 0.186890 & 2.819718 \\
\hline 1 & -5.251496 & -1.326742 & -1.172655 \\
\hline 1 & 4.996753 & -1.930874 & -2.094004 \\
\hline 1 & 4.846622 & -2.441076 & 2.145496 \\
\hline 6 & -3.441853 & 2.250831 & 2.434850 \\
\hline 1 & -2.716657 & 2.924104 & 1.968535 \\
\hline 6 & -3.659110 & 0.467978 & -2.375116 \\
\hline 1 & -2.708274 & 1.003651 & -2.455367 \\
\hline 6 & -6.152787 & -1.830011 & 1.401619 \\
\hline 1 & -6.886492 & -1.382024 & 2.085530 \\
\hline 6 & -2.727271 & 1.529698 & 3.583439 \\
\hline 1 & -1.908778 & 0.912534 & 3.204580 \\
\hline 1 & -2.316691 & 2.257246 & 4.290415 \\
\hline 1 & -3.419358 & 0.885668 & 4.136343 \\
\hline 6 & -4.596114 & 3.115217 & 2.955823 \\
\hline 1 & -5.081142 & 3.656127 & 2.138134 \\
\hline 1 & -5.355593 & 2.497840 & 3.446943 \\
\hline 1 & -4.231571 & 3.844723 & 3.685734 \\
\hline 6 & -3.454946 & -0.915767 & -3.003011 \\
\hline 1 & -4.406259 & -1.441525 & -3.138762 \\
\hline
\end{tabular}




$\begin{array}{lrrr}1 & -3.004179 & -0.804117 & -3.993795 \\ 1 & -2.798499 & -1.538593 & -2.394066 \\ 6 & -4.727749 & 1.226095 & -3.175604 \\ 1 & -4.458630 & 1.266750 & -4.235952 \\ 1 & -5.693289 & 0.715694 & -3.088306 \\ 1 & -4.860785 & 2.249353 & -2.818005 \\ 6 & -5.292112 & -2.809265 & 2.213312 \\ 1 & -4.526929 & -3.252778 & 1.567855 \\ 1 & -4.779439 & -2.303432 & 3.035978 \\ 1 & -5.904500 & -3.616238 & 2.629002 \\ 6 & -6.919695 & -2.580793 & 0.314501 \\ 1 & -7.609607 & -3.298132 & 0.767506 \\ 1 & -7.498032 & -1.900153 & -0.316645 \\ 1 & -6.236659 & -3.146016 & -0.329193 \\ 6 & 3.410103 & -0.256197 & 2.786232 \\ 1 & 2.685646 & 0.554153 & 2.659804 \\ 6 & 3.700786 & 0.399743 & -2.293981 \\ 1 & 3.177077 & 1.322566 & -2.029870 \\ 6 & 5.851543 & -3.595708 & -0.101368 \\ 1 & 5.945393 & -4.018059 & 0.907135 \\ 6 & 5.165041 & -4.640280 & -0.987022 \\ 1 & 5.764755 & -5.553979 & -1.039511 \\ 1 & 4.176230 & -4.904055 & -0.600648 \\ 1 & 5.039129 & -4.266088 & -2.008774 \\ 6 & 7.262287 & -3.292535 & -0.619799 \\ 1 & 7.219578 & -2.880110 & -1.633413 \\ 1 & 7.766156 & -2.561942 & 0.018200 \\ 1 & 7.868298 & -4.203066 & -0.651633 \\ 6 & 2.763934 & -0.418416 & -3.190523 \\ 1 & -0.873816 & -4.976926 & -0.421067 \\ 1 & -1.874736 & -5.472108 & 0.523965 \\ 1 & -1.785354 & -4.749483 & 1.865330 \\ 6 & -2.037176 & -3.692036 & 1.773729 \\ 1 & & & \end{array}$




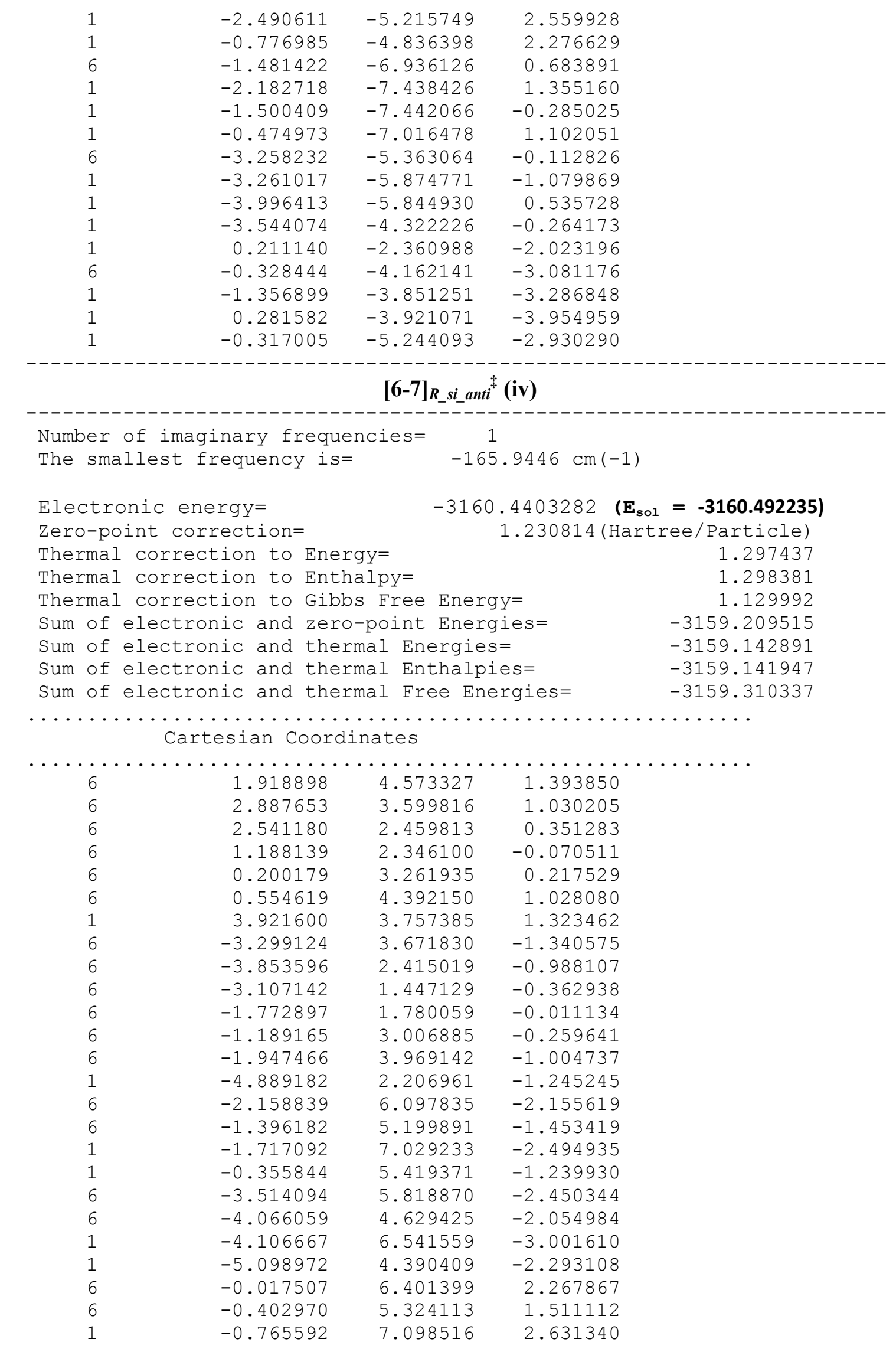




\begin{tabular}{|c|c|c|c|}
\hline 1 & -1.451455 & 5.169716 & 1.282094 \\
\hline 6 & 1.344197 & 6.608095 & 2.586588 \\
\hline 6 & 2.286601 & 5.708079 & 2.163779 \\
\hline 1 & 1.635704 & 7.468935 & 3.179306 \\
\hline 1 & 3.333675 & 5.838759 & 2.423073 \\
\hline 8 & -1.065008 & 0.817596 & 0.677138 \\
\hline 8 & 0.868774 & 1.246612 & -0.848096 \\
\hline 15 & 0.131680 & 0.029185 & -0.084133 \\
\hline 8 & -0.371770 & -0.887058 & -1.172607 \\
\hline 8 & 0.949180 & -0.569077 & 1.028157 \\
\hline 1 & -0.305947 & -2.222877 & -0.878016 \\
\hline 6 & -3.673591 & 0.093041 & -0.069733 \\
\hline 6 & -4.037012 & -0.756832 & -1.137456 \\
\hline 6 & -3.899271 & -0.318442 & 1.258304 \\
\hline 6 & -4.709553 & -1.946734 & -0.854564 \\
\hline 6 & -4.552235 & -1.532827 & 1.488535 \\
\hline 6 & -4.994961 & -2.346998 & 0.450104 \\
\hline 6 & 3.485954 & 1.338170 & 0.049610 \\
\hline 6 & 3.868292 & 0.442658 & 1.070473 \\
\hline 6 & 3.911472 & 1.124622 & -1.273821 \\
\hline 6 & 4.682256 & -0.640554 & 0.734965 \\
\hline 6 & 4.733494 & 0.033213 & -1.555633 \\
\hline 6 & 5.120773 & -0.867494 & -0.567570 \\
\hline 8 & -0.421883 & -3.231905 & -0.530165 \\
\hline 1 & 5.065211 & -0.121509 & -2.580071 \\
\hline 1 & 4.972067 & -1.347817 & 1.508403 \\
\hline 1 & -4.747871 & -1.840246 & 2.514364 \\
\hline 1 & -5.014618 & -2.583435 & -1.683304 \\
\hline 6 & 3.509773 & 2.047510 & -2.413884 \\
\hline 1 & 2.801665 & 2.786862 & -2.027675 \\
\hline 6 & 3.427681 & 0.623997 & 2.516496 \\
\hline 1 & 2.487347 & 1.184010 & 2.510383 \\
\hline 6 & 6.021462 & -2.047167 & -0.881453 \\
\hline 1 & 5.959776 & -2.736679 & -0.028898 \\
\hline 6 & 2.806908 & 1.282046 & -3.540766 \\
\hline 1 & 1.948257 & 0.726071 & -3.156087 \\
\hline 1 & 2.454755 & 1.978196 & -4.308330 \\
\hline 1 & 3.489652 & 0.575202 & -4.023770 \\
\hline 6 & 4.727862 & 2.814776 & -2.941632 \\
\hline 1 & 5.204446 & 3.391397 & -2.143621 \\
\hline 1 & 5.475082 & 2.126355 & -3.350520 \\
\hline 1 & 4.432864 & 3.504229 & -3.738785 \\
\hline 6 & 3.164110 & -0.699284 & 3.244068 \\
\hline 1 & 4.096341 & -1.228567 & 3.468598 \\
\hline 1 & 2.669206 & -0.499001 & 4.199022 \\
\hline 1 & 2.522276 & -1.355150 & 2.654149 \\
\hline 6 & 4.475432 & 1.426404 & 3.301573 \\
\hline 1 & 4.153663 & 1.568831 & 4.338220 \\
\hline 1 & 5.429613 & 0.888235 & 3.311873 \\
\hline 1 & 4.653388 & 2.410075 & 2.861912 \\
\hline 6 & 7.478835 & -1.587624 & -1.012956 \\
\hline 1 & 7.579021 & -0.882470 & -1.845048 \\
\hline 1 & 7.814751 & -1.082503 & -0.103370 \\
\hline 1 & 8.141890 & -2.437258 & -1.204753 \\
\hline 6 & 5.582425 & -2.810304 & -2.134529 \\
\hline 1 & 6.175558 & -3.722852 & -2.251790 \\
\hline 1 & 4.525699 & -3.087377 & -2.082166 \\
\hline 7 & 5.727879 & -2.207200 & -3.036643 \\
\hline
\end{tabular}




\begin{tabular}{|c|c|c|c|}
\hline 6 & -3.700809 & -0.444141 & -2.589428 \\
\hline 1 & -3.052647 & 0.436290 & -2.606957 \\
\hline 6 & -3.483898 & 0.509543 & 2.465088 \\
\hline 1 & -3.020252 & 1.434321 & 2.112185 \\
\hline 6 & -5.778281 & -3.616352 & 0.729877 \\
\hline D & -5.888312 & -3.697581 & 1.818854 \\
\hline 6 & -7.181714 & -3.537607 & 0.117987 \\
\hline 1 & -7.767453 & -4.424444 & 0.377968 \\
\hline 1 & -7.715864 & -2.652083 & 0.471763 \\
\hline 1 & -7.124460 & -3.481207 & -0.974021 \\
\hline 6 & -5.045638 & -4.868295 & 0.236971 \\
\hline 1 & -4.878116 & -4.822229 & -0.844852 \\
\hline 1 & -4.073634 & -4.979092 & 0.726515 \\
\hline 1 & -5.634703 & -5.766668 & 0.445117 \\
\hline 6 & -2.442973 & -0.240142 & 3.305928 \\
\hline 1 & -2.858478 & -1.173845 & 3.702165 \\
\hline 1 & -1.556814 & -0.471631 & 2.708149 \\
\hline 1 & -2.128828 & 0.371377 & 4.157345 \\
\hline 6 & -4.698667 & 0.904923 & 3.313726 \\
\hline 1 & -5.438582 & 1.442509 & 2.714231 \\
\hline 1 & -5.188145 & 0.024902 & 3.743414 \\
\hline 1 & -4.390120 & 1.550860 & 4.141088 \\
\hline 6 & -4.964273 & -0.131327 & -3.399150 \\
\hline 1 & -4.706857 & 0.110823 & -4.434597 \\
\hline 1 & -5.638244 & -0.994717 & -3.412612 \\
\hline 1 & -5.515599 & 0.713459 & -2.976697 \\
\hline 6 & -2.917858 & -1.593994 & -3.235703 \\
\hline 1 & -2.636215 & -1.325406 & -4.258437 \\
\hline 1 & -2.004388 & -1.801284 & -2.673823 \\
\hline 1 & -3.518189 & -2.508720 & -3.288393 \\
\hline 6 & -1.711919 & -3.298177 & 0.105870 \\
\hline 6 & -0.094791 & -3.530115 & 1.869289 \\
\hline 6 & 0.787382 & -3.662721 & 0.632091 \\
\hline 1 & 1.511206 & -1.857491 & 0.719425 \\
\hline 8 & 1.779024 & -2.823755 & 0.446820 \\
\hline 6 & -1.477880 & -4.005810 & 1.437387 \\
\hline 1 & -1.470771 & -5.094037 & 1.311187 \\
\hline 1 & -2.252563 & -3.739220 & 2.162016 \\
\hline 1 & -2.402579 & -3.822753 & -0.558322 \\
\hline 1 & -2.079081 & -2.275286 & 0.260696 \\
\hline 8 & 1.043979 & -4.939317 & 0.347174 \\
\hline 6 & 2.043351 & -5.323426 & -0.649835 \\
\hline 6 & 1.839851 & -4.572901 & -1.962523 \\
\hline 1 & 2.013756 & -3.501922 & -1.844368 \\
\hline 1 & 2.550520 & -4.961276 & -2.698494 \\
\hline 1 & 0.825604 & -4.725469 & -2.338097 \\
\hline 6 & 1.760934 & -6.809938 & -0.836345 \\
\hline 1 & 2.463105 & -7.235700 & -1.557424 \\
\hline 1 & 1.870408 & -7.338380 & 0.114461 \\
\hline 1 & 0.743011 & -6.959880 & -1.204721 \\
\hline 6 & 3.445498 & -5.119010 & -0.077606 \\
\hline 1 & 3.516984 & -5.592489 & 0.906072 \\
\hline 1 & 4.176619 & -5.590697 & -0.74193 \\
\hline 1 & 3.687665 & -4.060298 & 0.019566 \\
\hline 1 & -0.137738 & -2.456892 & 2.091818 \\
\hline 6 & 0.509420 & -4.280869 & 3.050250 \\
\hline 1 & 1.528969 & -3.935766 & 3.24545 \\
\hline 1 & -0.086980 & -4.108556 & 3.94933 \\
\hline
\end{tabular}




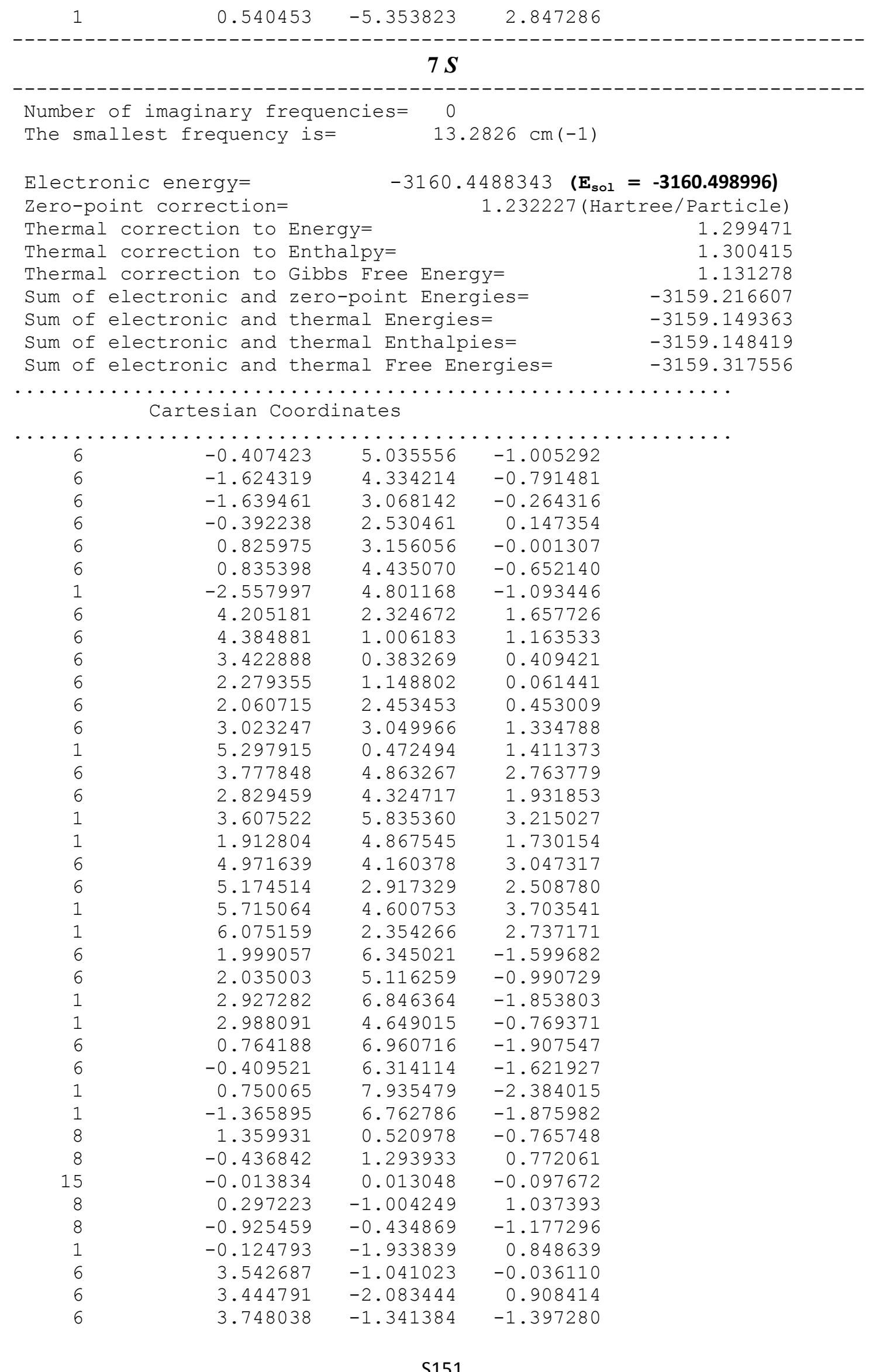

S151 


\begin{tabular}{|c|c|c|c|}
\hline 6 & 3.625596 & -3.399596 & 0.475492 \\
\hline 6 & 3.911780 & -2.672995 & -1.779720 \\
\hline 6 & 3.866621 & -3.717167 & -0.858160 \\
\hline 6 & -2.857694 & 2.204647 & -0.178747 \\
\hline 6 & -3.423092 & 1.686417 & -1.364113 \\
\hline 6 & -3.377184 & 1.828749 & 1.073155 \\
\hline 6 & -4.522507 & 0.834409 & -1.264732 \\
\hline 6 & -4.473580 & 0.966473 & 1.117770 \\
\hline 6 & -5.060124 & 0.456286 & -0.035835 \\
\hline 8 & -0.594960 & -3.217703 & 0.374056 \\
\hline 1 & -4.881165 & 0.674326 & 2.083905 \\
\hline 1 & -4.963455 & 0.438894 & -2.176339 \\
\hline 1 & 4.085999 & -2.896625 & -2.830089 \\
\hline 1 & 3.558399 & -4.208342 & 1.199882 \\
\hline 6 & -2.802136 & 2.337893 & 2.385892 \\
\hline 1 & -1.902527 & 2.921805 & 2.168039 \\
\hline 6 & -2.875364 & 2.014632 & -2.746542 \\
\hline 1 & -1.865200 & 2.417342 & -2.625798 \\
\hline 6 & -6.252794 & -0.476120 & 0.044326 \\
\hline 1 & -6.363544 & -0.769474 & 1.096898 \\
\hline 6 & -2.391854 & 1.185920 & 3.31013 \\
\hline 1 & -1.660693 & 0.537628 & 2.820862 \\
\hline 1 & -1.945344 & 1.578722 & 4.228982 \\
\hline 1 & -3.256400 & 0.576769 & 3.595547 \\
\hline 6 & -3.798374 & 3.274042 & 3.081284 \\
\hline 1 & -4.067395 & 4.113410 & 2.433714 \\
\hline 1 & -4.718848 & 2.740325 & 3.339749 \\
\hline 1 & -3.370242 & 3.673805 & 4.005754 \\
\hline 6 & -2.759601 & 0.779059 & -3.646214 \\
\hline 1 & -3.742779 & 0.393521 & -3.935163 \\
\hline 1 & -2.233556 & 1.043916 & -4.568555 \\
\hline 1 & -2.201886 & -0.011514 & -3.140614 \\
\hline 6 & -3.740218 & 3.083866 & -3.426825 \\
\hline 1 & -3.340281 & 3.334652 & -4.414345 \\
\hline 1 & -4.763475 & 2.715132 & -3.557322 \\
\hline 1 & -3.792258 & 4.000985 & -2.833598 \\
\hline 6 & -6.031842 & -1.749749 & -0.776916 \\
\hline 1 & -6.028231 & -1.528709 & -1.850241 \\
\hline 1 & -5.072650 & -2.207559 & -0.522328 \\
\hline 1 & -6.834996 & -2.470695 & -0.59276 \\
\hline 6 & -7.536211 & 0.242381 & -0.38745 \\
\hline 1 & -8.401578 & -0.422368 & -0.301965 \\
\hline 1 & -7.718726 & 1.130291 & 0.224073 \\
\hline 1 & -7.458432 & 0.563859 & -1.43169 \\
\hline 6 & 3.124313 & -1.831890 & 2.37545 \\
\hline 1 & 2.673327 & -0.838045 & 2.453057 \\
\hline 6 & 3.809052 & -0.268308 & -2.473209 \\
\hline 1 & 3.615575 & 0.702914 & -2.009362 \\
\hline 6 & 4.090407 & -5.155078 & -1.28571 \\
\hline 1 & 3.821106 & -5.791859 & -0.43325 \\
\hline 6 & 3.209988 & -5.557819 & -2.472722 \\
\hline 1 & 3.340299 & -6.619327 & -2.702762 \\
\hline 1 & 2.151160 & -5.379093 & -2.263808 \\
\hline 1 & 3.474619 & -4.990837 & -3.370762 \\
\hline 6 & 5.570925 & -5.393511 & -1.60726 \\
\hline 1 & 5.880535 & -4.774717 & -2.45625 \\
\hline 1 & 6.203826 & -5.134259 & -0.754578 \\
\hline 1 & 5.750216 & -6.441189 & -1.86775 \\
\hline
\end{tabular}




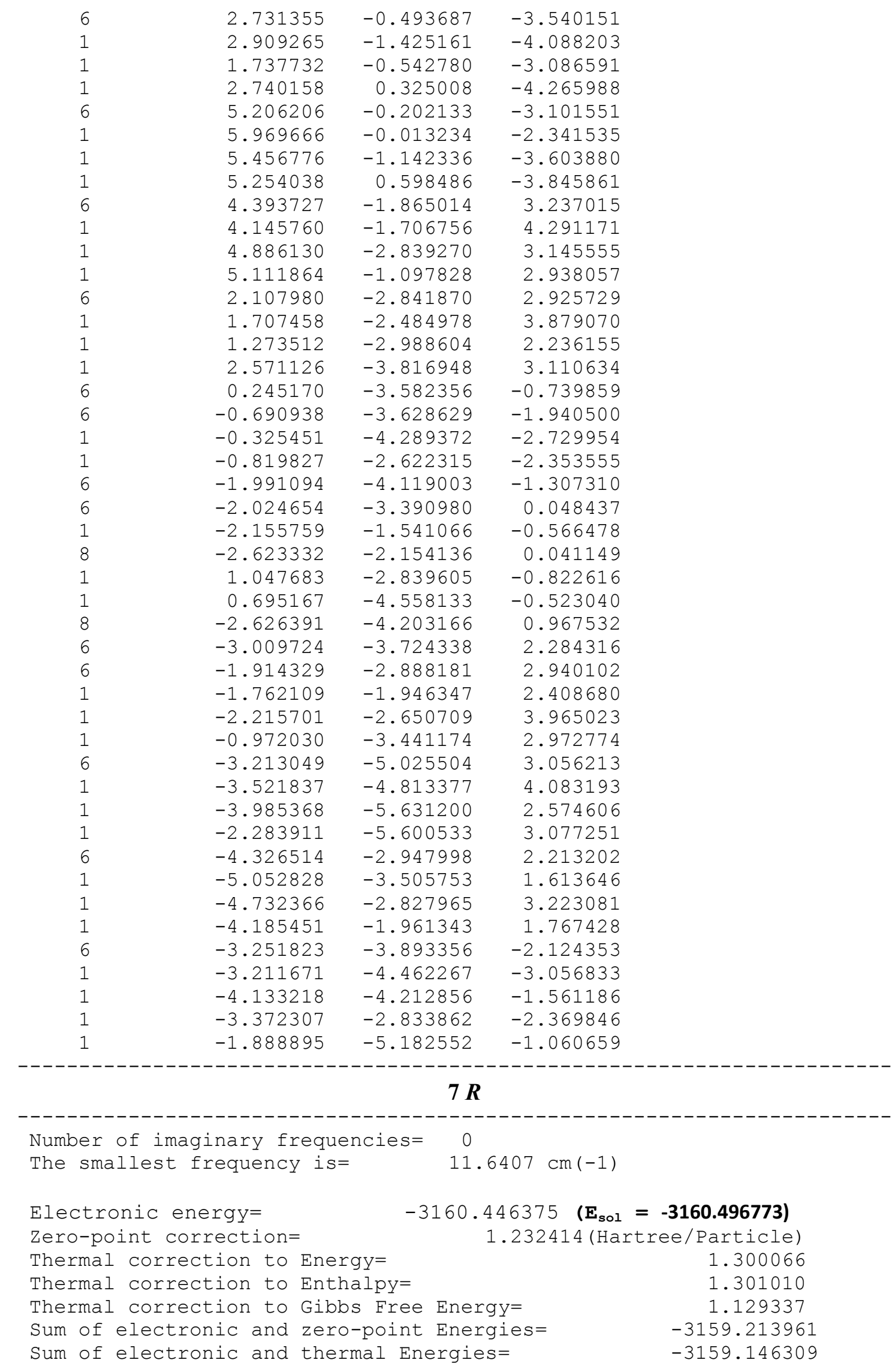


$\begin{array}{lll}\text { Sum of electronic and thermal Enthalpies= } & -3159.145365 \\ \text { Sum of electronic and thermal Free Energies= } & -3159.317038\end{array}$

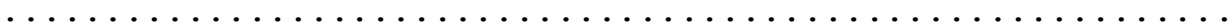

Cartesian Coordinates

\begin{tabular}{|c|c|c|c|}
\hline 6 & 3.853619 & 3.172412 & 1.445791 \\
\hline 6 & 4.288782 & 1.865845 & 1.097504 \\
\hline 6 & 3.481248 & 0.997748 & 0.408370 \\
\hline 6 & 2.225985 & 1.497497 & -0.029891 \\
\hline 6 & 1.755494 & 2.764711 & 0.226046 \\
\hline 6 & 2.565909 & 3.624505 & 1.040919 \\
\hline 1 & 5.278130 & 1.542953 & 1.408122 \\
\hline 6 & -1.104734 & 4.698736 & -1.470596 \\
\hline 6 & -2.190232 & 3.859021 & -1.113792 \\
\hline 6 & -2.000850 & 2.676991 & -0.441822 \\
\hline 6 & -0.674519 & 2.354693 & -0.053632 \\
\hline 6 & 0.418098 & 3.162646 & -0.295404 \\
\hline 6 & 0.217958 & 4.344141 & -1.083514 \\
\hline 1 & -3.197844 & 4.152912 & -1.395780 \\
\hline 6 & 1.056571 & 6.289424 & -2.271178 \\
\hline 6 & 1.292270 & 5.163567 & -1.524161 \\
\hline 1 & 1.891710 & 6.897890 & -2.602622 \\
\hline 1 & 2.309007 & 4.883737 & -1.271893 \\
\hline 6 & -0.262693 & 6.661606 & -2.620447 \\
\hline 6 & -1.316743 & 5.878292 & -2.231219 \\
\hline 1 & -0.434202 & 7.558899 & -3.205905 \\
\hline 1 & -2.334613 & 6.138345 & -2.508370 \\
\hline 6 & 2.932901 & 5.689541 & 2.264740 \\
\hline 6 & 2.122822 & 4.896401 & 1.492189 \\
\hline 1 & 2.573049 & 6.654998 & 2.605118 \\
\hline 1 & 1.127045 & 5.234047 & 1.227284 \\
\hline 6 & 4.228243 & 5.257281 & 2.630415 \\
\hline 6 & 4.672822 & 4.023794 & 2.232579 \\
\hline 1 & 4.859828 & 5.896777 & 3.238209 \\
\hline 1 & 5.656147 & 3.667523 & 2.526545 \\
\hline 8 & -0.514578 & 1.185645 & 0.673479 \\
\hline 8 & 1.440732 & 0.637926 & -0.792203 \\
\hline 15 & 0.241574 & -0.073182 & 0.003300 \\
\hline 8 & -0.613790 & -0.636958 & -1.167251 \\
\hline 8 & 0.609032 & -1.021400 & 1.080151 \\
\hline 1 & -1.066728 & -1.527066 & -0.888591 \\
\hline 6 & -3.154874 & 1.781477 & -0.119921 \\
\hline 6 & -3.860895 & 1.147231 & -1.165119 \\
\hline 6 & -3.582593 & 1.625151 & 1.211811 \\
\hline 6 & -5.036474 & 0.459550 & -0.862657 \\
\hline 6 & -4.748952 & 0.897691 & 1.464771 \\
\hline 6 & -5.508329 & 0.334868 & 0.443681 \\
\hline 6 & 3.845455 & -0.420452 & 0.104145 \\
\hline 6 & 3.781676 & -1.400898 & 1.114716 \\
\hline 6 & 4.175076 & -0.779885 & -1.216029 \\
\hline 6 & 4.072635 & -2.723493 & 0.775385 \\
\hline 6 & 4.461974 & -2.113387 & -1.501517 \\
\hline 6 & 4.408260 & -3.101414 & -0.521381 \\
\hline 8 & -1.849585 & -2.606237 & -0.282742 \\
\hline 1 & 4.713607 & -2.390364 & -2.523465 \\
\hline 1 & 4.011688 & -3.492881 & 1.540591 \\
\hline 1 & -5.091140 & 0.787961 & 2.492200 \\
\hline 1 & -5.593809 & -0.006158 & -1.673115 \\
\hline
\end{tabular}




\begin{tabular}{|c|c|c|c|}
\hline 6 & 4.228194 & 0.236564 & -2.346127 \\
\hline 1 & 3.896035 & 1.204764 & -1.959234 \\
\hline 6 & 3.410227 & -1.065308 & 2.553029 \\
\hline 1 & 2.799478 & -0.156965 & 2.536310 \\
\hline 6 & 4.641981 & -4.557933 & -0.870355 \\
\hline 1 & 4.604121 & -5.127660 & 0.066592 \\
\hline 6 & 3.285318 & -0.146570 & -3.492289 \\
\hline 1 & 2.263406 & -0.273458 & -3.126683 \\
\hline 1 & 3.286776 & 0.632720 & -4.260706 \\
\hline 1 & 3.600139 & -1.081335 & -3.967457 \\
\hline 6 & 5.666103 & 0.413503 & -2.848180 \\
\hline 1 & 6.329382 & 0.727394 & -2.037030 \\
\hline- & 6.054571 & -0.525962 & -3.255092 \\
\hline 1 & 5.708116 & 1.166953 & -3.640858 \\
\hline 6 & 2.577859 & -2.157900 & 3.234262 \\
\hline 1 & 3.183395 & -3.040128 & 3.467044 \\
\hline 1 & 2.184597 & -1.778235 & 4.181993 \\
\hline 1 & 1.735273 & -2.463630 & 2.612163 \\
\hline 6 & 4.670708 & -0.798701 & 3.387750 \\
\hline 1 & 4.403634 & -0.559016 & 4.421858 \\
\hline 1 & 5.308474 & -1.689283 & 3.398638 \\
\hline 1 & 5.261471 & 0.028507 & 2.987944 \\
\hline 6 & 6.015766 & -4.784588 & -1.508592 \\
\hline 1 & 6.094740 & -4.254304 & -2.463284 \\
\hline 1 & 6.817969 & -4.426635 & -0.857889 \\
\hline 1 & 6.178263 & -5.848573 & -1.705800 \\
\hline 6 & 3.521708 & -5.071465 & -1.783414 \\
\hline 1 & 3.630100 & -6.144778 & -1.968993 \\
\hline 1 & 2.540333 & -4.889567 & -1.336705 \\
\hline 1 & 3.549790 & -4.556737 & -2.750028 \\
\hline 6 & -3.371653 & 1.154965 & -2.607134 \\
\hline 1 & -2.353955 & 1.556302 & -2.617490 \\
\hline 6 & -2.839372 & 2.224896 & 2.394909 \\
\hline 1 & -1.963838 & 2.763684 & 2.023476 \\
\hline 6 & -6.804633 & -0.393555 & 0.744887 \\
\hline 1 & -6.975714 & -0.316939 & 1.826114 \\
\hline 6 & -7.989958 & 0.266980 & 0.032255 \\
\hline 1 & -8.927983 & -0.222647 & 0.310493 \\
\hline 1 & -8.062558 & 1.327129 & 0.288308 \\
\hline 1 & -7.883007 & 0.189790 & -1.054712 \\
\hline 6 & -6.716939 & -1.881004 & 0.387345 \\
\hline 1 & -6.520955 & -2.013906 & -0.682533 \\
\hline 1 & -5.914618 & -2.375233 & 0.942973 \\
\hline 1 & -7.657050 & -2.390565 & 0.619549 \\
\hline 6 & -2.340343 & 1.123242 & 3.337577 \\
\hline 1 & -3.180833 & 0.578403 & 3.781574 \\
\hline 1 & -1.704442 & 0.411591 & 2.802827 \\
\hline 1 & -1.756274 & 1.556860 & 4.154997 \\
\hline 6 & -3.712372 & 3.239686 & 3.142804 \\
\hline 1 & -4.056930 & 4.031301 & 2.47141 \\
\hline 1 & -4.594800 & 2.759202 & 3.577683 \\
\hline 1 & -3.147296 & 3.700809 & 3.958317 \\
\hline 6 & -4.251061 & 2.044486 & -3.494065 \\
\hline 1 & -3.893368 & 2.029404 & -4.528002 \\
\hline 1 & -5.286795 & 1.687860 & -3.48915 \\
\hline 1 & -4.254268 & 3.082040 & -3.14967 \\
\hline 6 & -3.307521 & -0.265630 & -3.182862 \\
\hline 1 & -2.832885 & -0.247632 & -4.16831 \\
\hline
\end{tabular}




\begin{tabular}{|c|c|c|c|}
\hline 1 & -2.725451 & -0.928149 & -2.537886 \\
\hline 1 & -4.307096 & -0.694598 & -3.306941 \\
\hline 6 & -3.001224 & -2.000405 & 0.340738 \\
\hline 6 & -1.727790 & -3.140610 & 2.001678 \\
\hline 6 & -1.252339 & -3.604838 & 0.618159 \\
\hline 1 & 0.461784 & -2.743839 & 0.688012 \\
\hline 8 & 0.101658 & -3.616020 & 0.419506 \\
\hline 6 & -3.152763 & -2.679357 & 1.703508 \\
\hline 1 & -3.811106 & -3.551690 & 1.635668 \\
\hline 1 & -3.552841 & -1.988471 & 2.450356 \\
\hline 1 & -3.862699 & -2.130865 & -0.318478 \\
\hline 1 & -2.800437 & -0.925993 & 0.444054 \\
\hline 8 & -1.815285 & -4.828540 & 0.361220 \\
\hline 6 & -1.406582 & -5.646888 & -0.762589 \\
\hline 6 & -1.129924 & -4.835240 & -2.027688 \\
\hline 1 & -0.278478 & -4.165714 & -1.895075 \\
\hline 1 & -0.904173 & -5.529217 & -2.843031 \\
\hline 1 & -2.004663 & -4.242495 & -2.305196 \\
\hline 6 & -2.613622 & -6.554775 & -0.980355 \\
\hline 1 & -2.409383 & -7.275325 & -1.777022 \\
\hline 1 & -2.842245 & -7.102353 & -0.062082 \\
\hline 1 & -3.488364 & -5.960285 & -1.257216 \\
\hline 6 & -0.192134 & -6.476890 & -0.348687 \\
\hline 1 & -0.424346 & -7.036842 & 0.561942 \\
\hline 1 & 0.063196 & -7.188894 & -1.139848 \\
\hline 1 & 0.666681 & -5.835102 & -0.153418 \\
\hline 1 & -1.112167 & -2.266272 & 2.248729 \\
\hline 6 & -1.576901 & -4.207072 & 3.072811 \\
\hline 1 & -0.537612 & -4.545049 & 3.123996 \\
\hline 1 & -1.858403 & -3.812395 & 4.052461 \\
\hline 1 & -2.205497 & -5.070423 & 2.844298 \\
\hline
\end{tabular}

\section{7'S}

Number of imaginary frequencies $=1$

The smallest frequency is $=-7.6942 \mathrm{~cm}(-1)$

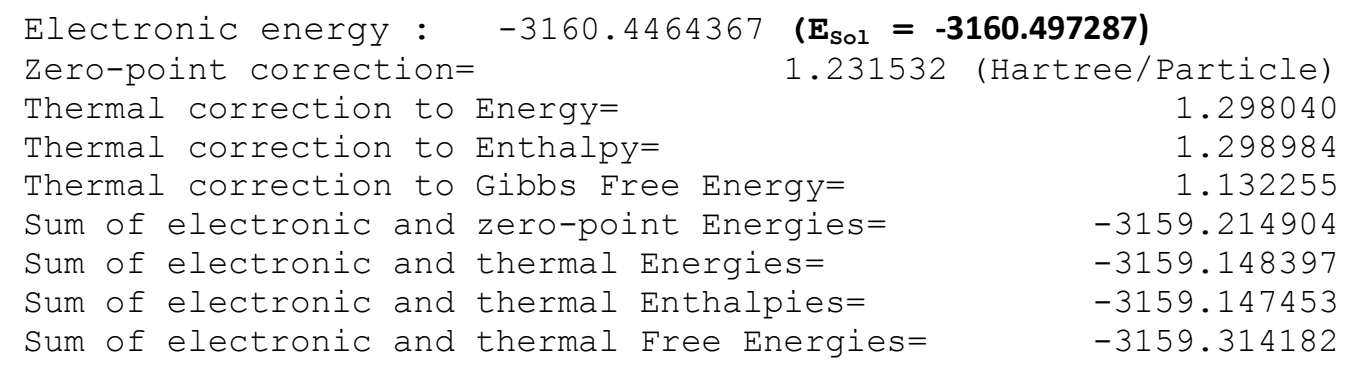

\begin{tabular}{|c|c|c|c|}
\hline 6 & -3.130099 & 3.716133 & -1.467866 \\
\hline 6 & -3.808796 & 2.539797 & -1.054388 \\
\hline 6 & -3.165529 & 1.530970 & -0.381760 \\
\hline 6 & -1.808080 & 1.754899 & -0.033992 \\
\hline 6 & -1.102144 & 2.895030 & -0.348162 \\
\hline 6 & -1.755421 & 3.892100 & -1.144325 \\
\hline 1 & -4.860713 & 2.428207 & -1.301652 \\
\hline 6 & 2.079545 & 4.240471 & 1.326866 \\
\hline 6 & 2.966727 & 3.180956 & 1.017184 \\
\hline
\end{tabular}




\begin{tabular}{|c|c|c|c|}
\hline 6 & 2.550453 & 2.046763 & 0.362407 \\
\hline 6 & 1.203844 & 2.010101 & -0.082472 \\
\hline 6 & 0.297127 & 3.028322 & 0.141759 \\
\hline 6 & 0.718701 & 4.155588 & 0.922434 \\
\hline 1 & 4.004775 & 3.256516 & 1.330592 \\
\hline 6 & 0.279463 & 6.245985 & 2.079492 \\
\hline 6 & -0.172517 & 5.180255 & 1.344401 \\
\hline 1 & -0.419012 & 7.013466 & 2.397004 \\
\hline 1 & -1.223386 & 5.107169 & 1.089085 \\
\hline 6 & 1.643766 & 6.351626 & 2.438081 \\
\hline 6 & 2.520340 & 5.364747 & 2.074076 \\
\hline 1 & 1.985859 & 7.204885 & 3.014521 \\
\hline 1 & 3.566751 & 5.415218 & 2.362626 \\
\hline 6 & -1.750540 & 5.973697 & -2.393256 \\
\hline 6 & -1.082161 & 5.038068 & -1.645411 \\
\hline 1 & -1.217808 & 6.839960 & -2.772107 \\
\hline 1 & -0.025700 & 5.162630 & -1.433956 \\
\hline 6 & -3.126475 & 5.817686 & -2.681084 \\
\hline 6 & -3.796398 & 4.710686 & -2.231049 \\
\hline 1 & -3.643701 & 6.569639 & -3.267888 \\
\hline 1 & -4.848280 & 4.566248 & -2.461707 \\
\hline 8 & 0.814814 & 0.903624 & -0.824257 \\
\hline 8 & -1.170433 & 0.767719 & 0.702869 \\
\hline 15 & -0.170529 & -0.178146 & -0.143794 \\
\hline 8 & 0.608168 & -0.868880 & 1.017165 \\
\hline 8 & -0.839490 & -1.003990 & -1.169882 \\
\hline 1 & -0.630650 & -2.714823 & -1.522822 \\
\hline 6 & 3.527899 & 0.930149 & 0.163211 \\
\hline 6 & 3.984416 & 0.217637 & 1.291919 \\
\hline 6 & 4.104081 & 0.694876 & -1.096426 \\
\hline 6 & 5.098001 & -0.610151 & 1.154258 \\
\hline 6 & 5.201367 & -0.166362 & -1.189254 \\
\hline 6 & 5.739914 & -0.795309 & -0.070284 \\
\hline 6 & -3.834243 & 0.239632 & -0.033958 \\
\hline 6 & -4.184042 & -0.664952 & -1.058690 \\
\hline 6 & -4.105502 & -0.076680 & 1.309788 \\
\hline 6 & -4.848100 & -1.842575 & -0.715183 \\
\hline 6 & -4.760695 & -1.274424 & 1.601193 \\
\hline 6 & -5.145190 & -2.168308 & 0.606732 \\
\hline 8 & 1.617308 & -2.540211 & -1.121720 \\
\hline 1 & -4.977458 & -1.521496 & 2.639237 \\
\hline 1 & -5.128896 & -2.533159 & -1.506411 \\
\hline 1 & 5.651748 & -0.329859 & -2.165278 \\
\hline 1 & 5.477369 & -1.139329 & 2.027276 \\
\hline 6 & -3.732866 & 0.843990 & 2.462000 \\
\hline 1 & -3.211986 & 1.716190 & 2.055974 \\
\hline 6 & -3.862485 & -0.409334 & -2.524905 \\
\hline 1 & -3.093320 & 0.367160 & -2.571473 \\
\hline 6 & -5.891167 & -3.441572 & 0.958362 \\
\hline 1 & -5.792241 & -3.584437 & 2.042599 \\
\hline 6 & -2.780046 & 0.155599 & 3.446310 \\
\hline 1 & -1.857659 & -0.149617 & 2.945070 \\
\hline 1 & -2.518058 & 0.838602 & 4.260466 \\
\hline 1 & -3.244113 & -0.730958 & 3.892102 \\
\hline 6 & -4.988455 & 1.356635 & 3.177797 \\
\hline 1 & -5.659395 & 1.863353 & 2.478366 \\
\hline 1 & -5.542857 & 0.532537 & 3.638573 \\
\hline 7 & -4.717106 & 2.061769 & 3.969487 \\
\hline
\end{tabular}




\begin{tabular}{|c|c|c|c|}
\hline 6 & -3.293207 & -1.649449 & -3.222345 \\
\hline 1 & -4.055868 & -2.422251 & -3.363327 \\
\hline 1 & -2.919795 & -1.376868 & -4.213725 \\
\hline 1 & -2.467495 & -2.072149 & -2.648925 \\
\hline 6 & -5.105240 & 0.081040 & -3.279891 \\
\hline 1 & -4.870256 & 0.259494 & -4.333847 \\
\hline 1 & -5.897971 & -0.673756 & -3.231677 \\
\hline 1 & -5.504270 & 1.007092 & -2.858600 \\
\hline 6 & -5.313363 & -4.679306 & 0.265603 \\
\hline 1 & -5.460153 & -4.630845 & -0.817907 \\
\hline 1 & -4.240581 & -4.779830 & 0.452368 \\
\hline 1 & -5.814328 & -5.583882 & 0.623328 \\
\hline 6 & -7.383061 & -3.291356 & 0.635233 \\
\hline 1 & -7.938136 & -4.189851 & 0.922466 \\
\hline 1 & -7.814447 & -2.433633 & 1.158163 \\
\hline 1 & -7.523100 & -3.134270 & -0.439606 \\
\hline 6 & 3.274857 & 0.288280 & 2.637252 \\
\hline 1 & 2.307883 & 0.776090 & 2.480375 \\
\hline 6 & 3.567273 & 1.337495 & -2.364060 \\
\hline 1 & 2.745504 & 2.005122 & -2.090482 \\
\hline 6 & 6.926884 & -1.737473 & -0.156051 \\
\hline 1 & 7.441978 & -1.689550 & 0.812282 \\
\hline 6 & 6.436628 & -3.177649 & -0.353493 \\
\hline 1 & 7.274178 & -3.882372 & -0.360966 \\
\hline 1 & 5.744492 & -3.469992 & 0.442570 \\
\hline 1 & 5.908319 & -3.265627 & -1.310193 \\
\hline 6 & 7.933035 & -1.359140 & -1.243257 \\
\hline 1 & 7.512208 & -1.508315 & -2.242852 \\
\hline 1 & 8.240340 & -0.313489 & -1.157221 \\
\hline 1 & 8.824168 & -1.988873 & -1.169770 \\
\hline 6 & 2.995474 & 0.262721 & -3.297135 \\
\hline 1 & 3.787094 & -0.412272 & -3.642531 \\
\hline 1 & 2.232526 & -0.331815 & -2.786231 \\
\hline 1 & 2.542153 & 0.724160 & -4.179778 \\
\hline 6 & 4.631395 & 2.184964 & -3.068886 \\
\hline 1 & 5.022828 & 2.958209 & -2.401666 \\
\hline 1 & 5.473802 & 1.570167 & -3.402490 \\
\hline 1 & 4.206143 & 2.673024 & -3.950965 \\
\hline 6 & 4.070684 & 1.099444 & 3.665787 \\
\hline 1 & 3.554263 & 1.108041 & 4.630642 \\
\hline 1 & 5.062907 & 0.660264 & 3.816244 \\
\hline 1 & 4.205013 & 2.135316 & 3.343913 \\
\hline 6 & 2.987217 & -1.117285 & 3.183181 \\
\hline 1 & 2.319817 & -1.053197 & 4.048175 \\
\hline 1 & 2.508562 & -1.746069 & 2.427151 \\
\hline 1 & 3.905281 & -1.615081 & 3.512936 \\
\hline 6 & 2.989309 & -2.813171 & -0.815108 \\
\hline 6 & 3.042700 & -4.290938 & -0.38456 \\
\hline 1 & 3.086084 & -4.365550 & 0.705405 \\
\hline 1 & 3.904315 & -4.816808 & -0.802502 \\
\hline 6 & 1.712434 & -4.850769 & -0.904818 \\
\hline 6 & 0.816842 & -3.619262 & -0.737804 \\
\hline 8 & -0.324935 & -3.648669 & -1.469182 \\
\hline 1 & 3.571037 & -2.608195 & -1.719775 \\
\hline 1 & 3.323839 & -2.127895 & -0.030532 \\
\hline 8 & 0.560189 & -3.365187 & 0.67612 \\
\hline 1 & 0.666595 & -1.887409 & 0.88631 \\
\hline 6 & 1.777251 & -5.256758 & -2.37457 \\
\hline
\end{tabular}




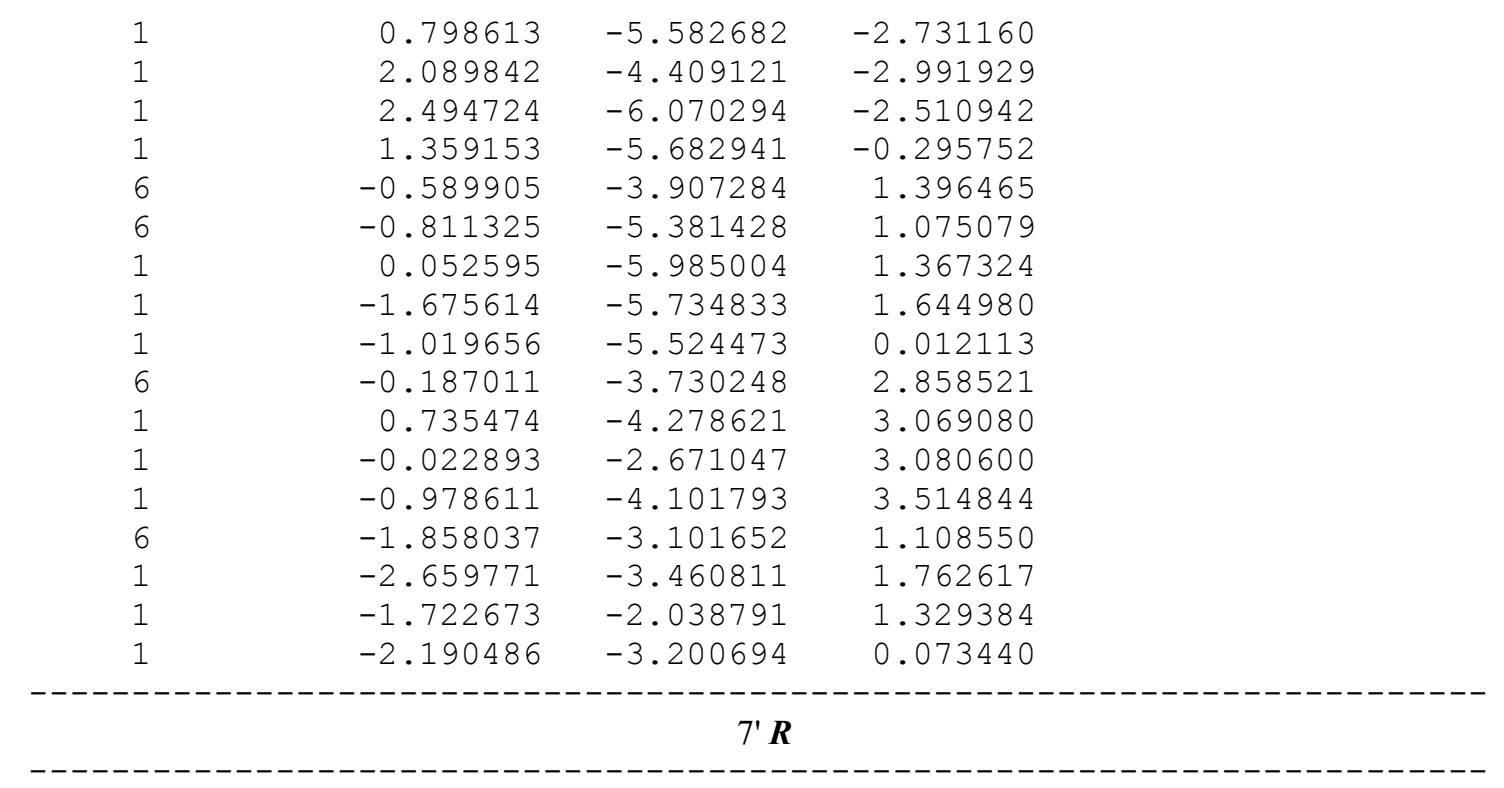

Number of imaginary frequencies $=0$

The smallest frequency is $=10.7930 \mathrm{~cm}(-1)$

\begin{tabular}{|c|c|c|}
\hline Electronic energy & \multicolumn{2}{|c|}{$-3160.4388146 \quad\left(E_{\mathrm{Sol}}=\mathbf{- 3 1 6 0 . 4 8 9 6 0 4}\right)$} \\
\hline Zero-point correction & 1.233154 & (Hartree/Particle) \\
\hline Thermal correction to & Energy= & 237 \\
\hline rmal correction to & Enthalpy= & 181 \\
\hline hermal correction to & Gibbs Free Ene & 1.132945 \\
\hline um of electronic and & zero-point Energies= & -3159.205661 \\
\hline jum of electronic & thermal Energies= & -3159.138578 \\
\hline Sum of electronic a & thermal Enthalpies= & -3159.137633 \\
\hline um of & Chermal Fre & -3159.305870 \\
\hline
\end{tabular}

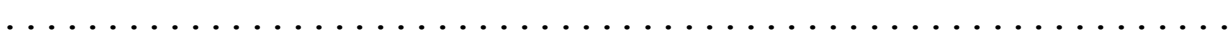

Cartesian Coordinates

$\begin{array}{rrrr}\ldots & \ldots \ldots \ldots \ldots \\ 6 & -3.123693 & 3.772922 & -1.348773 \\ 6 & -3.798736 & 2.561215 & -1.047414 \\ 6 & -3.159417 & 1.506020 & -0.445462 \\ 6 & -1.811492 & 1.708779 & -0.052725 \\ 6 & -1.112573 & 2.878285 & -0.255283 \\ 6 & -1.759063 & 3.933084 & -0.979297 \\ 1 & -4.846653 & 2.463737 & -1.317176 \\ 6 & 2.033067 & 4.110695 & 1.570028 \\ 6 & 2.940950 & 3.102419 & 1.163861 \\ 6 & 2.547791 & 2.028946 & 0.401566 \\ 6 & 1.199914 & 2.004286 & -0.039607 \\ 6 & 0.276846 & 2.984448 & 0.267479 \\ 6 & 0.676454 & 4.043702 & 1.148078 \\ 1 & 3.978397 & 3.170525 & 1.480995 \\ 6 & 0.197889 & 6.014952 & 2.484613 \\ 6 & -0.232745 & 5.014470 & 1.651304 \\ 1 & -0.513878 & 6.741491 & 2.862943 \\ 1 & -1.280063 & 4.950886 & 1.379171 \\ 6 & 1.557551 & 6.105282 & 2.864692 \\ 6 & 2.451331 & 5.167931 & 2.420354 \\ 1 & 1.883445 & 6.907215 & 3.518874 \\ 1 & 3.494783 & 5.207112 & 2.721029 \\ 6 & -1.749500 & 6.112984 & -2.047811\end{array}$




\begin{tabular}{|c|c|c|c|}
\hline 6 & -1.087443 & 5.123662 & -1.366591 \\
\hline 1 & -1.217428 & 7.012611 & -2.339655 \\
\hline 1 & -0.036970 & 5.240034 & -1.123593 \\
\hline 6 & -3.116913 & 5.969764 & -2.379359 \\
\hline 6 & -3.784171 & 4.822257 & -2.040505 \\
\hline 1 & -3.629129 & 6.763891 & -2.912532 \\
\hline 1 & -4.829030 & 4.688068 & -2.306625 \\
\hline 8 & 0.825833 & 0.956742 & -0.869986 \\
\hline 8 & -1.179903 & 0.671024 & 0.615808 \\
\hline 15 & -0.138630 & -0.191683 & -0.274302 \\
\hline 8 & 0.654970 & -0.910016 & 0.867095 \\
\hline 8 & -0.766754 & -0.972370 & -1.357606 \\
\hline 1 & -0.561181 & -2.766272 & -1.765711 \\
\hline 6 & 3.553338 & 0.972031 & 0.068367 \\
\hline 6 & 4.079801 & 0.175536 & 1.106430 \\
\hline 6 & 4.084895 & 0.875708 & -1.230121 \\
\hline 6 & 5.213023 & -0.598844 & 0.852678 \\
\hline 6 & 5.203462 & 0.066347 & -1.439788 \\
\hline 6 & 5.808382 & -0.644753 & -0.407301 \\
\hline 6 & -3.848011 & 0.202866 & -0.190871 \\
\hline 6 & -4.144881 & -0.655285 & -1.267672 \\
\hline 6 & -4.225775 & -0.147923 & 1.118895 \\
\hline 6 & -4.888677 & -1.809604 & -1.015402 \\
\hline 6 & -4.952937 & -1.321181 & 1.320941 \\
\hline 6 & -5.316175 & -2.153903 & 0.263835 \\
\hline 8 & 1.600548 & -2.471326 & -1.237651 \\
\hline 1 & -5.249390 & -1.584912 & 2.334546 \\
\hline 1 & -5.140023 & -2.468024 & -1.843884 \\
\hline 1 & 5.628746 & 0.000463 & -2.439852 \\
\hline 1 & 5.631542 & -1.192431 & 1.662510 \\
\hline 6 & -3.891880 & 0.717975 & 2.324566 \\
\hline 1 & -3.335412 & 1.594851 & 1.980505 \\
\hline 6 & -3.680894 & -0.368665 & -2.688566 \\
\hline 1 & -2.849473 & 0.340277 & -2.629531 \\
\hline 6 & -6.115684 & -3.422991 & 0.493393 \\
\hline 1 & -6.447148 & -3.776806 & -0.491005 \\
\hline 6 & -2.997440 & -0.031753 & 3.318951 \\
\hline 1 & -2.050658 & -0.316217 & 2.851925 \\
\hline 1 & -2.775669 & 0.601698 & 4.183560 \\
\hline 1 & -3.489562 & -0.939230 & 3.685875 \\
\hline 6 & -5.167794 & 1.228156 & 3.004857 \\
\hline 1 & -5.795702 & 1.778034 & 2.298056 \\
\hline 1 & -5.760149 & 0.399175 & 3.405931 \\
\hline 1 & -4.918439 & 1.893976 & 3.836685 \\
\hline 6 & -3.155355 & -1.625975 & -3.390392 \\
\hline 1 & -3.970132 & -2.300877 & -3.673518 \\
\hline 1 & -2.629406 & -1.346524 & -4.307560 \\
\hline 1 & -2.460516 & -2.171349 & -2.750142 \\
\hline 6 & -4.804212 & 0.253160 & -3.528300 \\
\hline 1 & -4.462946 & 0.429163 & -4.553322 \\
\hline 1 & -5.665067 & -0.423298 & -3.568827 \\
\hline 1 & -5.145917 & 1.205692 & -3.116684 \\
\hline 6 & -7.362161 & -3.179495 & 1.348547 \\
\hline 1 & -7.090754 & -2.888785 & 2.368407 \\
\hline 1 & -7.982807 & -2.385264 & 0.925742 \\
\hline 1 & -7.963807 & -4.090906 & 1.415488 \\
\hline 6 & -5.237062 & -4.516909 & 1.111364 \\
\hline 1 & -5.804147 & -5.443019 & 1.248673 \\
\hline
\end{tabular}




\begin{tabular}{|c|c|c|c|}
\hline 1 & -4.372685 & -4.729484 & 0.474941 \\
\hline 1 & -4.866113 & -4.198680 & 2.092997 \\
\hline 6 & 3.421249 & 0.088275 & 2.476514 \\
\hline 1 & 2.446929 & 0.582457 & 2.412561 \\
\hline 6 & 3.482728 & 1.611915 & -2.415227 \\
\hline 1 & 2.663483 & 2.240258 & -2.055501 \\
\hline 6 & 7.026437 & -1.507105 & -0.678732 \\
\hline 1 & 7.452034 & -1.172953 & -1.633387 \\
\hline 6 & 8.107226 & -1.360403 & 0.395337 \\
\hline 1 & 9.004705 & -1.917068 & 0.110583 \\
\hline 1 & 8.381357 & -0.312406 & 0.541291 \\
\hline 1 & 7.767106 & -1.758005 & 1.356812 \\
\hline 6 & 6.611115 & -2.974556 & -0.836086 \\
\hline 1 & 6.136833 & -3.335347 & 0.084129 \\
\hline 1 & 5.892488 & -3.089737 & -1.653369 \\
\hline 1 & 7.477707 & -3.609470 & -1.045923 \\
\hline 6 & 2.890447 & 0.605788 & -3.410340 \\
\hline 1 & 3.678378 & -0.023249 & -3.840447 \\
\hline 1 & 2.158646 & -0.042658 & -2.920068 \\
\hline 1 & 2.393371 & 1.128529 & -4.233130 \\
\hline 6 & 4.500583 & 2.535702 & -3.092579 \\
\hline 1 & 4.912092 & 3.257060 & -2.380993 \\
\hline 1 & 5.334227 & 1.967919 & -3.517817 \\
\hline 1 & 4.025592 & 3.088290 & -3.908738 \\
\hline 6 & 4.252648 & 0.784645 & 3.559450 \\
\hline 1 & 3.778127 & 0.672887 & 4.539164 \\
\hline 1 & 5.255021 & 0.346367 & 3.617504 \\
\hline 1 & 4.363719 & 1.852960 & 3.356350 \\
\hline 6 & 3.161510 & -1.373308 & 2.868170 \\
\hline 1 & 2.546707 & -1.414467 & 3.772645 \\
\hline 1 & 2.636144 & -1.910120 & 2.073366 \\
\hline 1 & 4.095546 & -1.904548 & 3.080590 \\
\hline 6 & 3.009604 & -2.674658 & -1.086787 \\
\hline 6 & 3.165388 & -4.129996 & -0.648100 \\
\hline 1 & 3.198276 & -4.203989 & 0.445028 \\
\hline 1 & 4.060137 & -4.606296 & -1.054237 \\
\hline 6 & 1.873254 & -4.768251 & -1.159058 \\
\hline 6 & 0.873784 & -3.626350 & -0.915747 \\
\hline 8 & -0.248722 & -3.692659 & -1.684787 \\
\hline 1 & 3.479637 & -2.478610 & -2.057757 \\
\hline 1 & 3.394876 & -1.953506 & -0.362073 \\
\hline 8 & 0.563137 & -3.466900 & 0.497290 \\
\hline 1 & 0.717796 & -1.912491 & 0.724212 \\
\hline 6 & 1.604918 & -6.139369 & -0.570083 \\
\hline 1 & 2.412212 & -6.818302 & -0.859539 \\
\hline 1 & 1.584959 & -6.090790 & 0.521821 \\
\hline 1 & 0.664059 & -6.563451 & -0.925951 \\
\hline 1 & 1.918356 & -4.842242 & -2.25280 \\
\hline 6 & -0.684553 & -3.886523 & 1.152288 \\
\hline 6 & -1.129711 & -5.293610 & 0.76625 \\
\hline 1 & -0.426883 & -6.052847 & 1.10990 \\
\hline 1 & -2.090010 & -5.486462 & 1.254093 \\
\hline 1 & -1.270406 & -5.377326 & -0.31283 \\
\hline 6 & -0.302563 & -3.834100 & 2.629625 \\
\hline 1 & 0.535064 & -4.508076 & 2.829861 \\
\hline 1 & -0.009333 & -2.817166 & 2.909341 \\
\hline 1 & -1.154003 & -4.131080 & 3.248277 \\
\hline 6 & -1.829976 & -2.907719 & 0.88641 \\
\hline
\end{tabular}




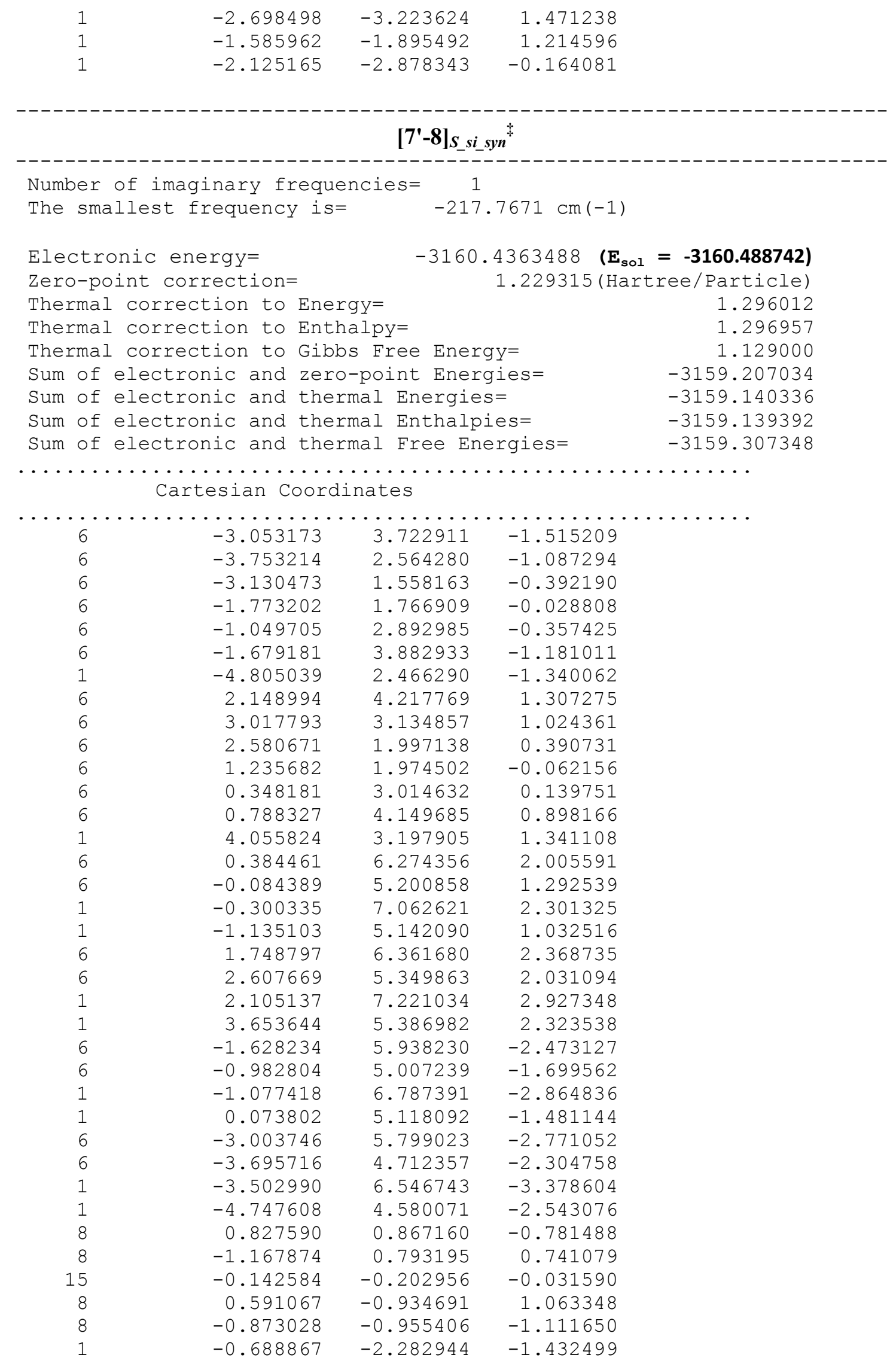




\begin{tabular}{|c|c|c|c|}
\hline 6 & 3.532209 & 0.855166 & 0.209148 \\
\hline 6 & 3.938761 & 0.116413 & 1.339060 \\
\hline 6 & 4.127676 & 0.612847 & -1.040545 \\
\hline 6 & 5.024398 & -0.751975 & 1.214680 \\
\hline 6 & 5.196172 & -0.284554 & -1.120691 \\
\hline 6 & 5.685898 & -0.946557 & 0.002625 \\
\hline 6 & -3.818462 & 0.280351 & -0.031237 \\
\hline 6 & -4.167038 & -0.637906 & -1.044753 \\
\hline 6 & -4.102794 & -0.014186 & 1.314663 \\
\hline 6 & -4.837881 & -1.807773 & -0.688516 \\
\hline 6 & -4.764024 & -1.205652 & 1.619629 \\
\hline 6 & -5.143177 & -2.113592 & 0.636431 \\
\hline 8 & 1.572090 & -2.439896 & -1.199186 \\
\hline 1 & -4.988351 & -1.436425 & 2.659896 \\
\hline 1 & -5.115168 & -2.509701 & -1.470923 \\
\hline 1 & 5.661428 & -0.453718 & -2.089391 \\
\hline 1 & 5.367087 & -1.301929 & 2.089694 \\
\hline 6 & -3.741836 & 0.926054 & 2.454977 \\
\hline 1 & -3.217120 & 1.790998 & 2.039040 \\
\hline 6 & -3.831363 & -0.406167 & -2.511854 \\
\hline 1 & -3.032963 & 0.340035 & -2.559338 \\
\hline 6 & -5.888828 & -3.382986 & 1.002389 \\
\hline 1 & -5.794545 & -3.510655 & 2.088972 \\
\hline 6 & -2.800583 & 0.258044 & 3.464111 \\
\hline 1 & -1.866482 & -0.044373 & 2.983533 \\
\hline 1 & -2.559515 & 0.954610 & 4.273334 \\
\hline 1 & -3.265712 & -0.625916 & 3.914361 \\
\hline 6 & -5.006589 & 1.450650 & 3.145924 \\
\hline 1 & -5.669613 & 1.943423 & 2.429167 \\
\hline 1 & -5.565822 & 0.634874 & 3.615732 \\
\hline 1 & -4.745039 & 2.170845 & 3.927334 \\
\hline 6 & -3.309720 & -1.671533 & -3.202022 \\
\hline 1 & -4.109363 & -2.401123 & -3.368499 \\
\hline 1 & -2.897839 & -1.414105 & -4.182208 \\
\hline 1 & -2.525310 & -2.150322 & -2.614764 \\
\hline 6 & -5.051625 & 0.120899 & -3.279041 \\
\hline 1 & -4.804624 & 0.278442 & -4.333730 \\
\hline 1 & -5.870397 & -0.605337 & -3.226995 \\
\hline 1 & -5.420299 & 1.065164 & -2.871922 \\
\hline 6 & -5.303703 & -4.628518 & 0.329666 \\
\hline 1 & -5.451442 & -4.598490 & -0.754419 \\
\hline 1 & -4.229844 & -4.717816 & 0.517228 \\
\hline 1 & -5.797940 & -5.530782 & 0.702464 \\
\hline 6 & -7.379537 & -3.242339 & 0.670183 \\
\hline 1 & -7.933365 & -4.139071 & 0.96554 \\
\hline 1 & -7.816120 & -2.379700 & 1.18048 \\
\hline 1 & -7.514640 & -3.098476 & -0.407147 \\
\hline 6 & 3.214060 & 0.216534 & 2.673650 \\
\hline 1 & 2.256402 & 0.714169 & 2.493851 \\
\hline 6 & 3.639191 & 1.290029 & -2.30982 \\
\hline 1 & 2.832538 & 1.978409 & -2.042749 \\
\hline 6 & 6.841916 & -1.927138 & -0.075331 \\
\hline 1 & 7.278592 & -1.986747 & 0.929927 \\
\hline 6 & 6.324829 & -3.324413 & -0.440462 \\
\hline 1 & 7.138634 & -4.056406 & -0.452358 \\
\hline 1 & 5.568080 & -3.661680 & 0.275493 \\
\hline 1 & 5.868690 & -3.308392 & -1.437781 \\
\hline 6 & 7.944854 & -1.491342 & -1.0412 \\
\hline
\end{tabular}




\begin{tabular}{|c|c|c|c|}
\hline 1 & 7.599367 & -1.520371 & -2.079505 \\
\hline 1 & 8.282476 & -0.474322 & -0.825757 \\
\hline 1 & 8.803465 & -2.164764 & -0.965825 \\
\hline 6 & 3.052104 & 0.249200 & -3.271080 \\
\hline 1 & 3.826703 & -0.449487 & -3.609295 \\
\hline 1 & 2.255403 & -0.319398 & -2.782717 \\
\hline 1 & 2.635502 & 0.737861 & -4.157106 \\
\hline 6 & 4.745493 & 2.112455 & -2.978326 \\
\hline 1 & 5.145268 & 2.863255 & -2.290851 \\
\hline 1 & 5.575824 & 1.475324 & -3.300688 \\
\hline 1 & 4.357142 & 2.626225 & -3.862814 \\
\hline 6 & 4.012996 & 1.032845 & 3.695916 \\
\hline 1 & 3.486934 & 1.066357 & 4.654994 \\
\hline 1 & 4.996468 & 0.580051 & 3.864090 \\
\hline 1 & 4.167356 & 2.060722 & 3.357927 \\
\hline 6 & 2.896092 & -1.173603 & 3.238812 \\
\hline 1 & 2.221571 & -1.080152 & 4.095620 \\
\hline 1 & 2.406054 & -1.796290 & 2.486069 \\
\hline 1 & 3.800313 & -1.684306 & 3.587152 \\
\hline 6 & 2.918294 & -2.831767 & -0.864898 \\
\hline 6 & 2.861921 & -4.349489 & -0.602548 \\
\hline 1 & 2.877575 & -4.548002 & 0.470673 \\
\hline 1 & 3.695689 & -4.875961 & -1.072304 \\
\hline 6 & 1.509606 & -4.762225 & -1.200020 \\
\hline 6 & 0.729789 & -3.473696 & -1.048405 \\
\hline 8 & -0.448796 & -3.321437 & -1.547890 \\
\hline 1 & 3.541689 & -2.558620 & -1.719795 \\
\hline 1 & 3.231591 & -2.243489 & 0.001102 \\
\hline 8 & 0.536124 & -3.378558 & 0.742269 \\
\hline 1 & 0.610163 & -2.340220 & 0.911601 \\
\hline 6 & 1.585743 & -5.096005 & -2.692313 \\
\hline 1 & 0.588461 & -5.261984 & -3.104334 \\
\hline 1 & 2.050493 & -4.276053 & -3.248807 \\
\hline 1 & 2.185619 & -5.996787 & -2.841469 \\
\hline 1 & 1.037737 & -5.575521 & -0.651949 \\
\hline 6 & -0.615250 & -3.869743 & 1.492918 \\
\hline 6 & -0.867873 & -5.313100 & 1.082270 \\
\hline 1 & 0.014017 & -5.933816 & 1.269951 \\
\hline 1 & -1.696948 & -5.714917 & 1.671420 \\
\hline 1 & -1.141842 & -5.369119 & 0.024518 \\
\hline 6 & -0.195999 & -3.772272 & 2.958049 \\
\hline 1 & 0.707277 & -4.361856 & 3.138849 \\
\hline 1 & 0.007407 & -2.728164 & 3.216035 \\
\hline 1 & -0.996450 & -4.140576 & 3.606270 \\
\hline 6 & -1.861658 & -3.018971 & 1.247260 \\
\hline 1 & -2.678823 & -3.406338 & 1.864960 \\
\hline 1 & -1.703167 & -1.978152 & 1.541196 \\
\hline 1 & -2.184746 & -3.041940 & 0.203769 \\
\hline \multicolumn{4}{|c|}{$\left[7^{\prime}-8\right]_{R_{-} \text {si_anti }}{ }^{*}$} \\
\hline \multirow{2}{*}{\multicolumn{4}{|c|}{$\begin{array}{l}\text { Number of imaginary frequencies }= \\
\text { The smallest frequency is }=\end{array}$}} \\
\hline & & & \\
\hline \multicolumn{3}{|c|}{ Electronic energy= } & $4281387 \quad\left(E_{\mathrm{sol}}=-3160.480806\right)$ \\
\hline \multicolumn{3}{|c|}{ Zero-point correction= } & 1.230527 (Hartree/Particle) \\
\hline \multicolumn{3}{|c|}{ Thermal correction to Energy= } & 1.296822 \\
\hline \multicolumn{4}{|c|}{ Thermal correction to Enthalpy= } \\
\hline
\end{tabular}




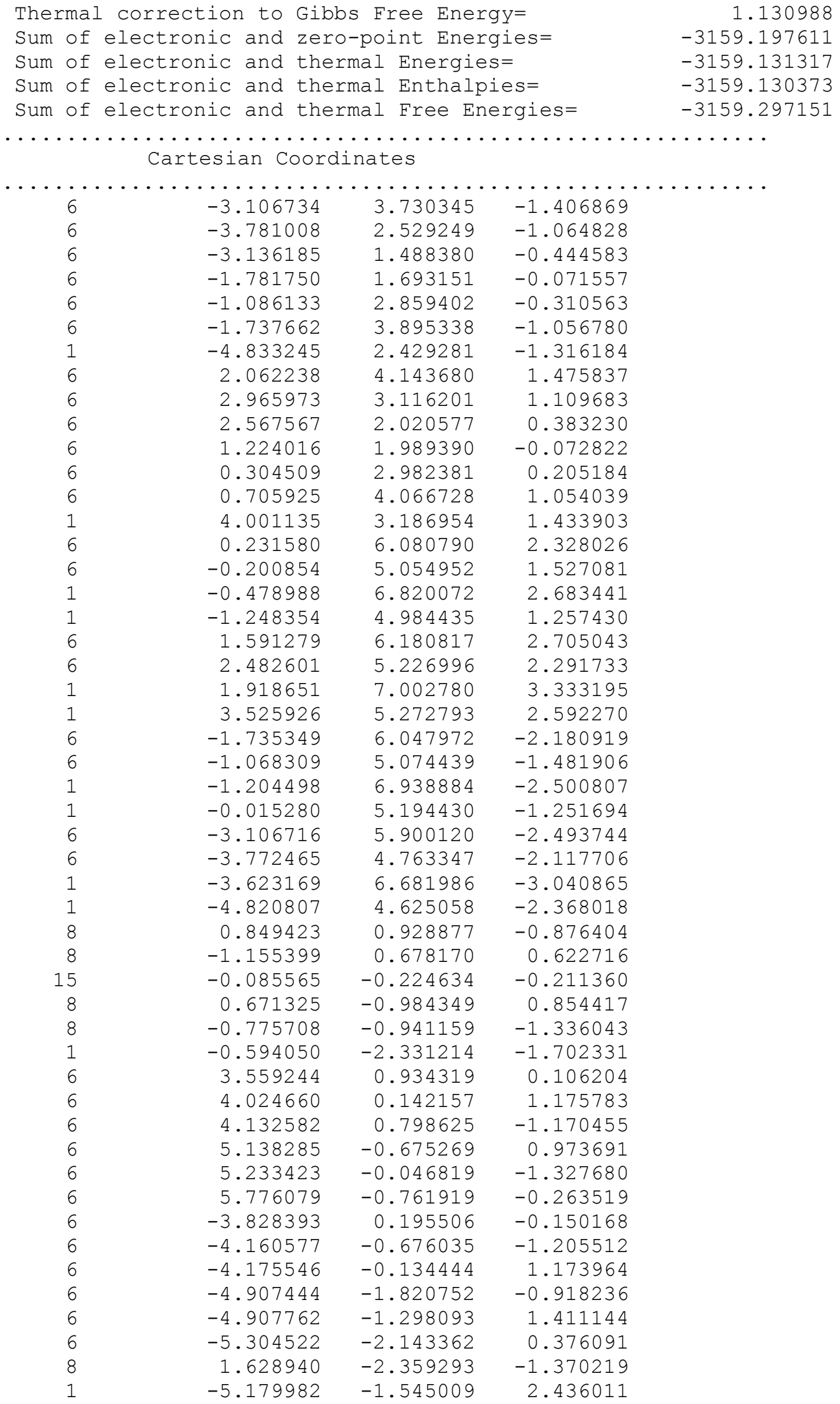




\begin{tabular}{|c|c|c|c|}
\hline 1 & -5.185312 & -2.488525 & -1.730877 \\
\hline 1 & 5.693416 & -0.139657 & -2.310625 \\
\hline 1 & 5.510025 & -1.265134 & 1.808305 \\
\hline 6 & -3.798543 & 0.740567 & 2.360322 \\
\hline & -3.247029 & 1.609570 & 1.989889 \\
\hline 6 & -3.726193 & -0.417368 & -2.641047 \\
\hline 1 & -2.887975 & 0.284991 & -2.611086 \\
\hline 6 & -6.115144 & -3.397361 & 0.647556 \\
\hline 1 & -6.444429 & -3.785627 & -0.324523 \\
\hline 6 & -2.877461 & -0.007417 & 3.331822 \\
\hline 1 & -1.947879 & -0.300755 & 2.836313 \\
\hline 1 & -2.624028 & 0.631807 & 4.183459 \\
\hline 1 & -3.364464 & -0.907880 & 3.723053 \\
\hline 6 & -5.048503 & 1.265471 & 3.076448 \\
\hline 1 & -5.697156 & 1.810126 & 2.384495 \\
\hline 1 & -5.631076 & 0.444814 & 3.507870 \\
\hline 1 & -4.768133 & 1.940190 & 3.891070 \\
\hline 6 & -3.227541 & -1.691822 & -3.332014 \\
\hline 1 & -4.056362 & -2.357652 & -3.596063 \\
\hline 1 & -2.709839 & -1.432716 & -4.259925 \\
\hline 1 & -2.534661 & -2.244615 & -2.695798 \\
\hline 6 & -4.862064 & 0.197262 & -3.469157 \\
\hline 1 & -4.541394 & 0.349274 & -4.504709 \\
\hline 1 & -5.729382 & -0.472158 & -3.477511 \\
\hline 1 & -5.186732 & 1.161072 & -3.070671 \\
\hline 6 & -7.363877 & -3.106611 & 1.484957 \\
\hline 1 & -7.093119 & -2.769314 & 2.490505 \\
\hline 1 & -7.976322 & -2.328213 & 1.022771 \\
\hline 1 & -7.972680 & -4.009506 & 1.592163 \\
\hline 6 & -5.253781 & -4.475777 & 1.315193 \\
\hline 1 & -5.835496 & -5.385502 & 1.494854 \\
\hline 1 & -4.392837 & -4.732162 & 0.690340 \\
\hline 1 & -4.877707 & -4.119196 & 2.281458 \\
\hline 6 & 3.327780 & 0.119329 & 2.529193 \\
\hline 1 & 2.360826 & 0.618009 & 2.414415 \\
\hline 6 & 3.589424 & 1.533603 & -2.384005 \\
\hline 1 & 2.773273 & 2.183447 & -2.056688 \\
\hline 6 & 6.974758 & -1.667374 & -0.478313 \\
\hline 1 & 7.471241 & -1.333266 & -1.398281 \\
\hline 6 & 7.993496 & -1.586977 & 0.660908 \\
\hline 1 & 8.890246 & -2.160660 & 0.409710 \\
\hline 1 & 8.287324 & -0.552612 & 0.856829 \\
\hline 1 & 7.587674 & -2.004334 & 1.587789 \\
\hline 6 & 6.514898 & -3.114601 & -0.693147 \\
\hline 1 & 5.950836 & -3.467201 & 0.178633 \\
\hline 1 & 5.865924 & -3.189928 & -1.571313 \\
\hline 1 & 7.369109 & -3.783092 & -0.840383 \\
\hline 6 & 3.003616 & 0.531100 & -3.385991 \\
\hline 1 & 3.786146 & -0.129982 & -3.777192 \\
\hline 1 & 2.231448 & -0.081278 & -2.911381 \\
\hline 1 & 2.554955 & 1.055784 & -4.234998 \\
\hline 6 & 4.651406 & 2.424521 & -3.036616 \\
\hline 1 & 5.056230 & 3.143602 & -2.318960 \\
\hline 1 & 5.485044 & 1.831841 & -3.427076 \\
\hline 1 & 4.218091 & 2.979537 & -3.874079 \\
\hline 6 & 4.139299 & 0.854608 & 3.601646 \\
\hline 1 & 3.632529 & 0.799645 & 4.570062 \\
\hline 1 & 5.130628 & 0.401375 & 3.71259 \\
\hline
\end{tabular}




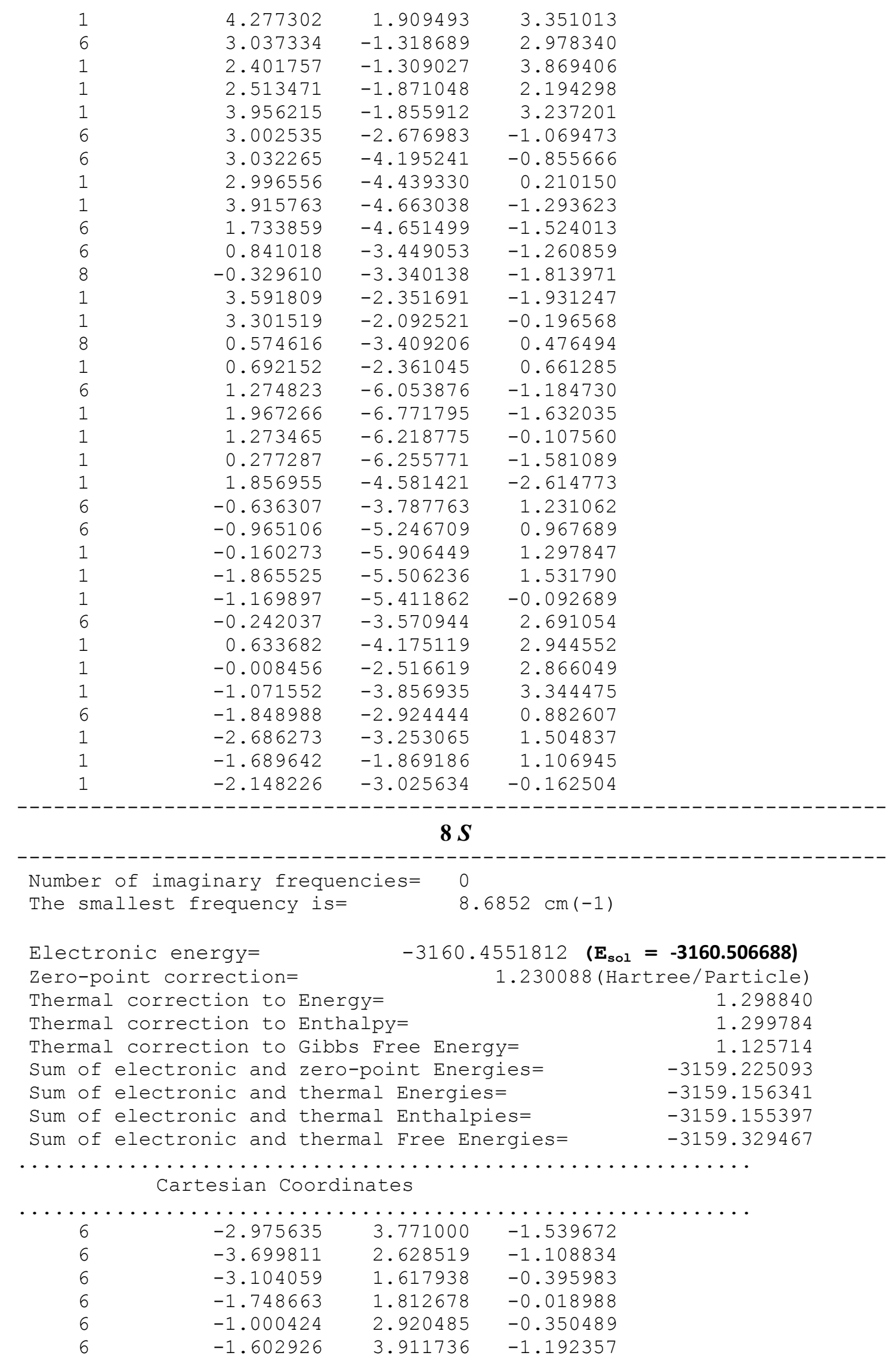




\begin{tabular}{|c|c|c|c|}
\hline 1 & -4.748634 & 2.543725 & -1.377629 \\
\hline 6 & 2.205493 & 4.206510 & 1.330057 \\
\hline 6 & 3.058575 & 3.106516 & 1.065034 \\
\hline 6 & 2.608368 & 1.969794 & 0.438727 \\
\hline 6 & 1.267952 & 1.968803 & -0.024946 \\
\hline 6 & 0.394727 & 3.023315 & 0.158405 \\
\hline 6 & 0.847536 & 4.158308 & 0.909473 \\
\hline 1 & 4.094418 & 3.154329 & 1.391113 \\
\hline 6 & 0.469363 & 6.298810 & 1.993697 \\
\hline 6 & -0.011275 & 5.227255 & 1.285731 \\
\hline 1 & -0.204309 & 7.101247 & 2.276268 \\
\hline 1 & -1.060437 & 5.183962 & 1.016578 \\
\hline 6 & 1.831763 & 6.366051 & 2.368488 \\
\hline 6 & 2.676814 & 5.337268 & 2.047791 \\
\hline 1 & 2.197498 & 7.224160 & 2.922862 \\
\hline 1 & 3.720403 & 5.359565 & 2.349722 \\
\hline 6 & -1.500812 & 5.949378 & -2.507860 \\
\hline 6 & -0.880405 & 5.016794 & -1.716038 \\
\hline 1 & -0.930906 & 6.783842 & -2.903680 \\
\hline 1 & 0.175636 & 5.111467 & -1.487534 \\
\hline 6 & -2.875244 & 5.830412 & -2.819489 \\
\hline 6 & -3.591791 & 4.761967 & -2.348402 \\
\hline 1 & -3.354113 & 6.579102 & -3.442037 \\
\hline 1 & -4.642964 & 4.645369 & -2.597499 \\
\hline 8 & 0.838561 & 0.853854 & -0.733015 \\
\hline 8 & -1.168364 & 0.835171 & 0.773206 \\
\hline 15 & -0.129218 & -0.171456 & 0.064873 \\
\hline 8 & 0.553927 & -0.974845 & 1.100983 \\
\hline 8 & -0.959100 & -0.838810 & -1.065633 \\
\hline 1 & -0.753641 & -1.783190 & -1.392620 \\
\hline 6 & 3.539871 & 0.809431 & 0.269766 \\
\hline 6 & 3.887151 & 0.033050 & 1.394270 \\
\hline 6 & 4.171796 & 0.584962 & -0.964979 \\
\hline 6 & 4.945969 & -0.869340 & 1.278644 \\
\hline 6 & 5.217058 & -0.339459 & -1.034635 \\
\hline 6 & 5.642243 & -1.051500 & 0.084374 \\
\hline 6 & -3.809458 & 0.346704 & -0.046085 \\
\hline 6 & -4.168802 & -0.557720 & -1.068059 \\
\hline 6 & -4.088597 & 0.036269 & 1.298414 \\
\hline 6 & -4.832534 & -1.734969 & -0.721325 \\
\hline 6 & -4.748395 & -1.157391 & 1.591611 \\
\hline 6 & -5.128416 & -2.057477 & 0.600802 \\
\hline 8 & 1.516666 & -2.361422 & -1.339100 \\
\hline 1 & -4.966011 & -1.401240 & 2.630279 \\
\hline 1 & -5.109041 & -2.428589 & -1.510541 \\
\hline 1 & 5.712891 & -0.493591 & -1.990599 \\
\hline 1 & 5.241892 & -1.453461 & 2.148094 \\
\hline 6 & -3.723606 & 0.959853 & 2.450796 \\
\hline 1 & -3.181412 & 1.821222 & 2.050170 \\
\hline 6 & -3.855515 & -0.308259 & -2.537556 \\
\hline 1 & -3.068639 & 0.449893 & -2.591381 \\
\hline 6 & -5.872180 & -3.327921 & 0.967713 \\
\hline 1 & -5.660243 & -3.528599 & 2.026472 \\
\hline 6 & -2.805826 & 0.265388 & 3.463717 \\
\hline 1 & -1.883960 & -0.077089 & 2.986472 \\
\hline 1 & -2.539544 & 0.957730 & 4.268604 \\
\hline 1 & -3.301407 & -0.598365 & 3.919967 \\
\hline 6 & -4.987621 & 1.498004 & 3.132902 \\
\hline
\end{tabular}




\begin{tabular}{|c|c|c|c|}
\hline 1 & -5.632075 & 2.013308 & 2.414984 \\
\hline 1 & -5.567379 & 0.685197 & 3.582448 \\
\hline 1 & -4.723746 & 2.201592 & 3.928545 \\
\hline 6 & -3.332123 & -1.560080 & -3.252257 \\
\hline & -4.128758 & -2.293212 & -3.416455 \\
\hline & -2.940457 & -1.285439 & -4.236274 \\
\hline 1 & -2.535917 & -2.049371 & -2.689230 \\
\hline 6 & -5.093862 & 0.213329 & -3.279686 \\
\hline ] & -4.864371 & 0.391462 & -4.335057 \\
\hline & -5.900655 & -0.526008 & -3.227632 \\
\hline & -5.472041 & 1.144629 & -2.851443 \\
\hline 6 & -5.421475 & -4.547334 & 0.159314 \\
\hline 1 & -5.711116 & -4.454919 & -0.892307 \\
\hline 1 & -4.336116 & -4.676933 & 0.200276 \\
\hline 1 & -5.894730 & -5.453930 & 0.548028 \\
\hline 6 & -7.384383 & -3.113567 & 0.821242 \\
\hline 1 & -7.938518 & -4.008389 & 1.121676 \\
\hline 1 & -7.723208 & -2.272433 & 1.432121 \\
\hline 1 & -7.632597 & -2.892117 & -0.222300 \\
\hline 6 & 3.143731 & 0.145952 & 2.717263 \\
\hline 1 & 2.197493 & 0.659902 & 2.522161 \\
\hline 6 & 3.748471 & 1.312722 & -2.229864 \\
\hline 1 & 2.944403 & 2.008672 & -1.973572 \\
\hline 6 & 6.767575 & -2.067439 & 0.015311 \\
\hline 1 & 7.164685 & -2.174823 & 1.032769 \\
\hline 6 & 6.216786 & -3.432539 & -0.416445 \\
\hline 1 & 7.005683 & -4.191249 & -0.428861 \\
\hline 1 & 5.424996 & -3.768630 & 0.260996 \\
\hline 1 & 5.795713 & -3.364565 & -1.426976 \\
\hline 6 & 7.919404 & -1.638417 & -0.895106 \\
\hline 1 & 7.612760 & -1.623863 & -1.945892 \\
\hline 1 & 8.281794 & -0.640676 & -0.634432 \\
\hline 1 & 8.751952 & -2.342586 & -0.809691 \\
\hline 6 & 3.183696 & 0.317220 & -3.250409 \\
\hline 1 & 3.956254 & -0.390345 & -3.574079 \\
\hline 1 & 2.352522 & -0.247005 & -2.816881 \\
\hline 1 & 2.820728 & 0.842786 & -4.138954 \\
\hline 6 & 4.897246 & 2.134675 & -2.822994 \\
\hline 1 & 5.277934 & 2.857221 & -2.095564 \\
\hline 1 & 5.730438 & 1.491833 & -3.125748 \\
\hline 1 & 4.559421 & 2.681767 & -3.708226 \\
\hline 6 & 3.944062 & 0.959790 & 3.741368 \\
\hline 1 & 3.408905 & 1.007034 & 4.694720 \\
\hline 1 & 4.918952 & 0.493530 & 3.922024 \\
\hline 1 & 4.116920 & 1.983028 & 3.398037 \\
\hline 6 & 2.798321 & -1.232492 & 3.294469 \\
\hline 1 & 2.115599 & -1.115481 & 4.141560 \\
\hline 1 & 2.307109 & -1.862608 & 2.549545 \\
\hline 1 & 3.690882 & -1.748857 & 3.663851 \\
\hline 6 & 2.847745 & -2.800341 & -0.997022 \\
\hline 6 & 2.747898 & -4.326333 & -0.837191 \\
\hline 1 & 2.606318 & -4.577707 & 0.214192 \\
\hline 1 & 3.637737 & -4.831262 & -1.220126 \\
\hline 6 & 1.483107 & -4.686679 & -1.627118 \\
\hline 6 & 0.718616 & -3.388492 & -1.605802 \\
\hline 8 & -0.458138 & -3.200542 & -1.869220 \\
\hline 1 & 3.507802 & -2.488203 & -1.811818 \\
\hline 1 & 3.131766 & -2.267472 & -0.08709 \\
\hline
\end{tabular}




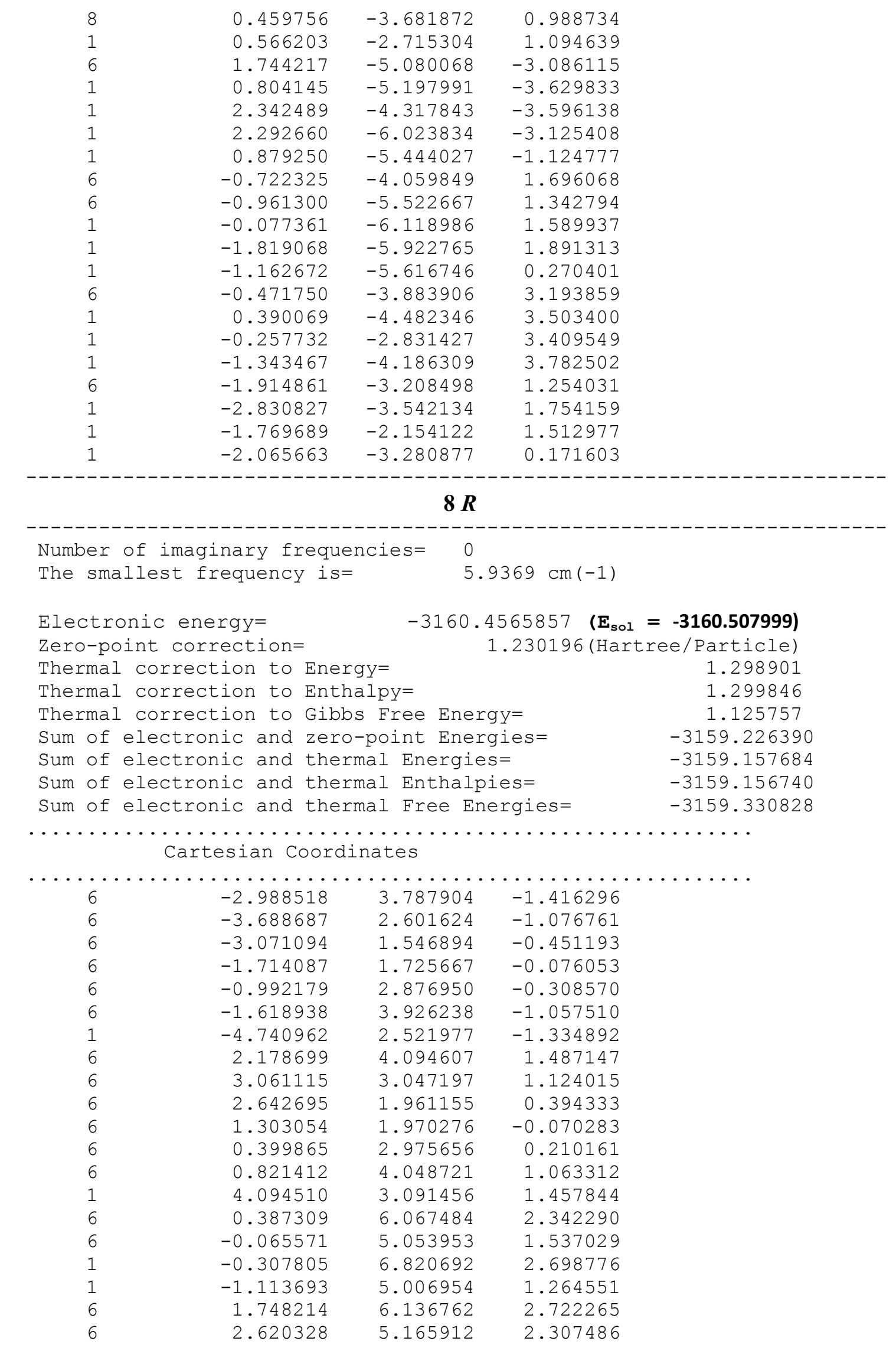




\begin{tabular}{|c|c|c|c|}
\hline 1 & 2.091600 & 6.948947 & 3.354487 \\
\hline 1 & 3.663488 & 5.188341 & 2.610851 \\
\hline 6 & -1.566118 & 6.077154 & -2.182678 \\
\hline 6 & -0.923096 & 5.091483 & -1.478337 \\
\hline 1 & -1.015812 & 6.957244 & -2.499386 \\
\hline 1 & 0.130635 & 5.191106 & -1.241904 \\
\hline 6 & -2.938015 & 5.955744 & -2.505079 \\
\hline 6 & -3.628947 & 4.833056 & -2.132843 \\
\hline 1 & -3.435236 & 6.747027 & -3.056391 \\
\hline 1 & -4.678001 & 4.715475 & -2.390233 \\
\hline 8 & 0.897055 & 0.917430 & -0.880768 \\
\hline 8 & -1.111571 & 0.688327 & 0.618561 \\
\hline 15 & -0.019827 & -0.205185 & -0.163778 \\
\hline 8 & 0.704628 & -1.050083 & 0.808749 \\
\hline 8 & -0.813115 & -0.820108 & -1.350952 \\
\hline 1 & -0.605896 & -1.750394 & -1.704506 \\
\hline 6 & 3.601859 & 0.841209 & 0.134066 \\
\hline 6 & 4.001920 & 0.016660 & 1.206642 \\
\hline 6 & 4.204645 & 0.698041 & -1.127350 \\
\hline 6 & 5.080499 & -0.849688 & 1.020224 \\
\hline 6 & 5.274387 & -0.190154 & -1.266522 \\
\hline 6 & 5.751482 & -0.945489 & -0.199202 \\
\hline 6 & -3.802040 & 0.273621 & -0.163404 \\
\hline 6 & -4.172507 & -0.573632 & -1.225532 \\
\hline 6 & -4.163877 & -0.052397 & 1.158230 \\
\hline 6 & -4.967810 & -1.687864 & -0.948065 \\
\hline 6 & -4.950463 & -1.181567 & 1.383772 \\
\hline 6 & -5.383303 & -2.000844 & 0.342154 \\
\hline 8 & 1.648867 & -2.314255 & -1.576271 \\
\hline 1 & -5.235845 & -1.425151 & 2.405880 \\
\hline 1 & -5.273719 & -2.335814 & -1.766525 \\
\hline 1 & 5.759817 & -0.286632 & -2.236774 \\
\hline 1 & 5.402487 & -1.467974 & 1.854854 \\
\hline 6 & -3.743080 & 0.787584 & 2.355130 \\
\hline 1 & -3.146995 & 1.632284 & 1.997536 \\
\hline 6 & -3.736276 & -0.322756 & -2.662129 \\
\hline 1 & -2.894263 & 0.375524 & -2.638508 \\
\hline 6 & -6.271448 & -3.202945 & 0.605351 \\
\hline 1 & -6.556728 & -3.614197 & -0.371268 \\
\hline 6 & -2.862935 & -0.025174 & 3.312172 \\
\hline 1 & -1.960804 & -0.377683 & 2.804771 \\
\hline 1 & -2.560960 & 0.588191 & 4.166933 \\
\hline 1 & -3.402795 & -0.896670 & 3.698735 \\
\hline 6 & -4.963468 & 1.367450 & 3.079654 \\
\hline 1 & -5.578538 & 1.961668 & 2.397885 \\
\hline 1 & -5.592463 & 0.572223 & 3.492506 \\
\hline 1 & -4.648475 & 2.008626 & 3.908550 \\
\hline 6 & -3.250397 & -1.605946 & -3.347801 \\
\hline 1 & -4.087834 & -2.262731 & -3.606387 \\
\hline 1 & -2.734027 & -1.357753 & -4.279797 \\
\hline 1 & -2.564205 & -2.170225 & -2.713830 \\
\hline 6 & -4.867035 & 0.300868 & -3.490517 \\
\hline 1 & -4.545387 & 0.450624 & -4.526083 \\
\hline 1 & -5.739839 & -0.361088 & -3.498955 \\
\hline 1 & -5.184219 & 1.267078 & -3.090919 \\
\hline 6 & -7.554926 & -2.802936 & 1.340629 \\
\hline 1 & -7.328899 & -2.422797 & 2.342219 \\
\hline 1 & -8.092489 & -2.020570 & 0.798657 \\
\hline
\end{tabular}




\begin{tabular}{|c|c|c|c|}
\hline 1 & -8.218326 & -3.665655 & 1.454396 \\
\hline 6 & -5.523511 & -4.297349 & 1.374980 \\
\hline 1 & -6.176016 & -5.156495 & 1.559625 \\
\hline 1 & -4.646883 & -4.640842 & 0.818643 \\
\hline 1 & -5.180537 & -3.920184 & 2.345314 \\
\hline & 3.286256 & 0.026171 & 2.550466 \\
\hline & 2.338541 & 0.557572 & 2.420144 \\
\hline 6 & 3.726266 & 1.472010 & -2.343902 \\
\hline ] & 2.916104 & 2.137411 & -2.031761 \\
\hline 6 & 6.922527 & -1.892890 & -0.383787 \\
\hline 1 & 7.434542 & -1.598911 & -1.308929 \\
\hline 6 & 7.935550 & -1.805574 & 0.760720 \\
\hline 1 & 8.815883 & -2.414730 & 0.536078 \\
\hline 1 & 8.260128 & -0.774710 & 0.923779 \\
\hline 1 & 7.509733 & -2.178235 & 1.697526 \\
\hline 6 & 6.425880 & -3.333319 & -0.558866 \\
\hline 1 & 5.834450 & -3.641468 & 0.311138 \\
\hline 1 & 5.794536 & -3.423066 & -1.448339 \\
\hline 1 & 7.264297 & -4.028780 & -0.665012 \\
\hline 6 & 3.150305 & 0.507882 & -3.388034 \\
\hline 1 & 3.927979 & -0.164630 & -3.768751 \\
\hline 1 & 2.349805 & -0.096641 & -2.951755 \\
\hline 1 & 2.743176 & 1.062530 & -4.23899 \\
\hline 6 & 4.835517 & 2.345689 & -2.938222 \\
\hline 1 & 5.227954 & 3.043214 & -2.192946 \\
\hline 1 & 5.669614 & 1.736817 & -3.30194 \\
\hline 1 & 4.452696 & 2.924366 & -3.784212 \\
\hline 6 & 4.110625 & 0.749749 & 3.622107 \\
\hline 1 & 3.589648 & 0.727513 & 4.584155 \\
\hline 1 & 5.082927 & 0.261894 & 3.752433 \\
\hline 1 & 4.291671 & 1.795129 & 3.35889 \\
\hline 6 & 2.945678 & -1.394489 & 3.01944 \\
\hline 1 & 2.301868 & -1.347521 & 3.903207 \\
\hline 1 & 2.412096 & -1.948934 & 2.244095 \\
\hline 1 & 3.844901 & -1.952296 & 3.301809 \\
\hline 6 & 2.966596 & -2.751810 & -1.176650 \\
\hline 6 & 2.834922 & -4.267875 & -0.99840 \\
\hline 1 & 2.520072 & -4.498770 & 0.022741 \\
\hline 1 & 3.764379 & -4.793542 & -1.22315 \\
\hline 6 & 1.692431 & -4.621283 & -1.95921 \\
\hline 6 & 0.889147 & -3.343900 & -1.937270 \\
\hline 8 & -0.282074 & -3.170257 & -2.225293 \\
\hline 1 & 3.658202 & -2.467025 & -1.976129 \\
\hline 1 & 3.218696 & -2.198629 & -0.27016 \\
\hline 8 & 0.372478 & -3.757984 & 0.77443 \\
\hline 1 & 0.607031 & -2.809632 & 0.775517 \\
\hline 6 & 0.869452 & -5.852844 & -1.621855 \\
\hline 1 & 1.471447 & -6.757511 & -1.734383 \\
\hline 1 & 0.519930 & -5.775166 & -0.59124 \\
\hline 1 & 0.002324 & -5.923357 & -2.28301 \\
\hline 1 & 2.082810 & -4.691668 & -2.98605 \\
\hline 6 & -0.809810 & -3.873716 & 1.572329 \\
\hline 6 & -1.157510 & -5.357269 & 1.60388 \\
\hline 1 & -0.297827 & -5.937158 & 1.95372 \\
\hline 1 & -2.001227 & -5.539601 & 2.276163 \\
\hline 1 & -1.434106 & -5.705845 & 0.60392 \\
\hline 6 & -0.509450 & -3.356825 & 2.979708 \\
\hline 1 & 0.301085 & -3.938266 & 3.43013 \\
\hline
\end{tabular}




$\begin{array}{lrrr}1 & -0.198168 & -2.308198 & 2.929373 \\ 1 & -1.393370 & -3.425628 & 3.622067 \\ 6 & -1.958016 & -3.081681 & 0.944989 \\ 1 & -2.891179 & -3.262630 & 1.486214 \\ 1 & -1.776122 & -2.003015 & 0.983243 \\ 1 & -2.097328 & -3.375201 & -0.101411\end{array}$

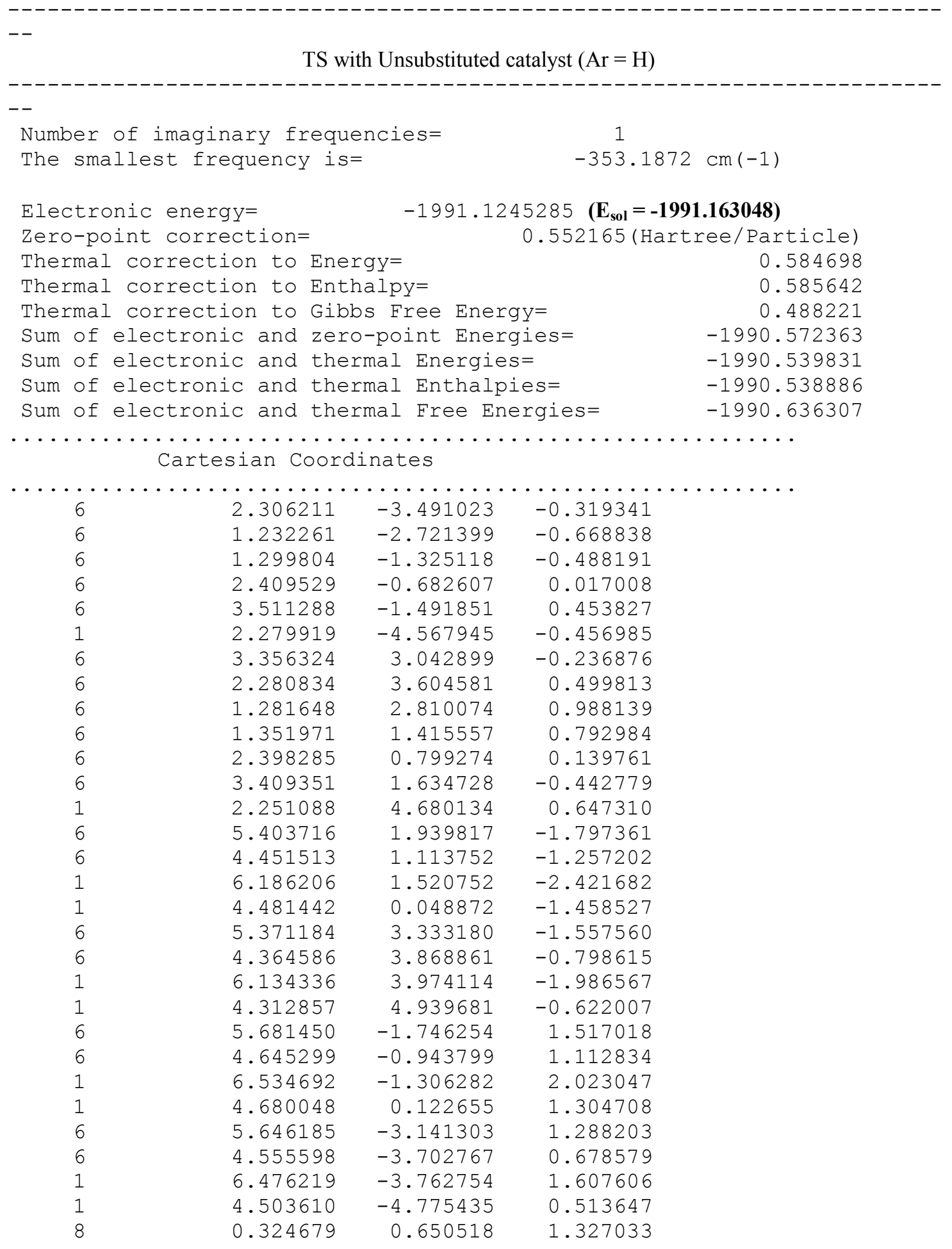




\begin{tabular}{|c|c|c|c|}
\hline 8 & 0.192769 & -0.579685 & -0.870071 \\
\hline 15 & -0.753342 & 0.007338 & 0.307290 \\
\hline 8 & -1.660372 & 1.041945 & -0.295299 \\
\hline 8 & -1.427410 & -1.130593 & 1.051969 \\
\hline 1 & -2.675016 & -0.920253 & 1.237404 \\
\hline 8 & -3.711223 & -0.580099 & 1.526132 \\
\hline 6 & -3.589277 & 0.410561 & 2.554663 \\
\hline 6 & -4.839695 & 1.271561 & 2.415763 \\
\hline 1 & -4.713315 & 2.251575 & 2.880435 \\
\hline 1 & -5.702461 & 0.774462 & 2.872326 \\
\hline 6 & -5.036196 & 1.365856 & 0.904408 \\
\hline 1 & -4.206863 & 1.942603 & 0.481226 \\
\hline 6 & -4.893267 & -0.081002 & 0.457061 \\
\hline 1 & -2.956638 & 0.500371 & -0.732664 \\
\hline 8 & -3.885456 & 0.106156 & -0.961386 \\
\hline 6 & -3.825208 & -0.920171 & -1.997831 \\
\hline 6 & -3.471249 & -2.267708 & -1.375423 \\
\hline 1 & -3.441722 & -3.031748 & -2.158082 \\
\hline 1 & -2.488533 & -2.226714 & -0.895884 \\
\hline 1 & -4.217358 & -2.555932 & -0.632030 \\
\hline 1 & -3.509952 & -0.093519 & 3.520200 \\
\hline 1 & -2.684666 & 1.006081 & 2.378873 \\
\hline 6 & -2.746369 & -0.467545 & -2.979246 \\
\hline 1 & -2.707999 & -1.166488 & -3.819501 \\
\hline 1 & -2.976994 & 0.529095 & -3.365315 \\
\hline 1 & -1.759644 & -0.439222 & -2.509220 \\
\hline 6 & -5.187723 & -0.944133 & -2.681702 \\
\hline 1 & -5.426277 & 0.052256 & -3.064894 \\
\hline 1 & -5.157888 & -1.640545 & -3.524548 \\
\hline 1 & -5.969876 & -1.256114 & -1.989660 \\
\hline 6 & 3.460751 & -2.901988 & 0.260243 \\
\hline 1 & 0.322983 & -3.143515 & -1.081659 \\
\hline 1 & 0.429447 & 3.211403 & 1.524565 \\
\hline 8 & -5.741340 & -0.899905 & 0.292197 \\
\hline 6 & -6.377268 & 1.917703 & 0.447571 \\
\hline 1 & -6.441042 & 1.909992 & -0.643502 \\
\hline 1 & -7.190760 & 1.302518 & 0.839448 \\
\hline 1 & -6.508138 & 2.944724 & 0.796500 \\
\hline
\end{tabular}

PRC with Unsubstituted catalyst $(\mathrm{Ar}=\mathrm{H})$

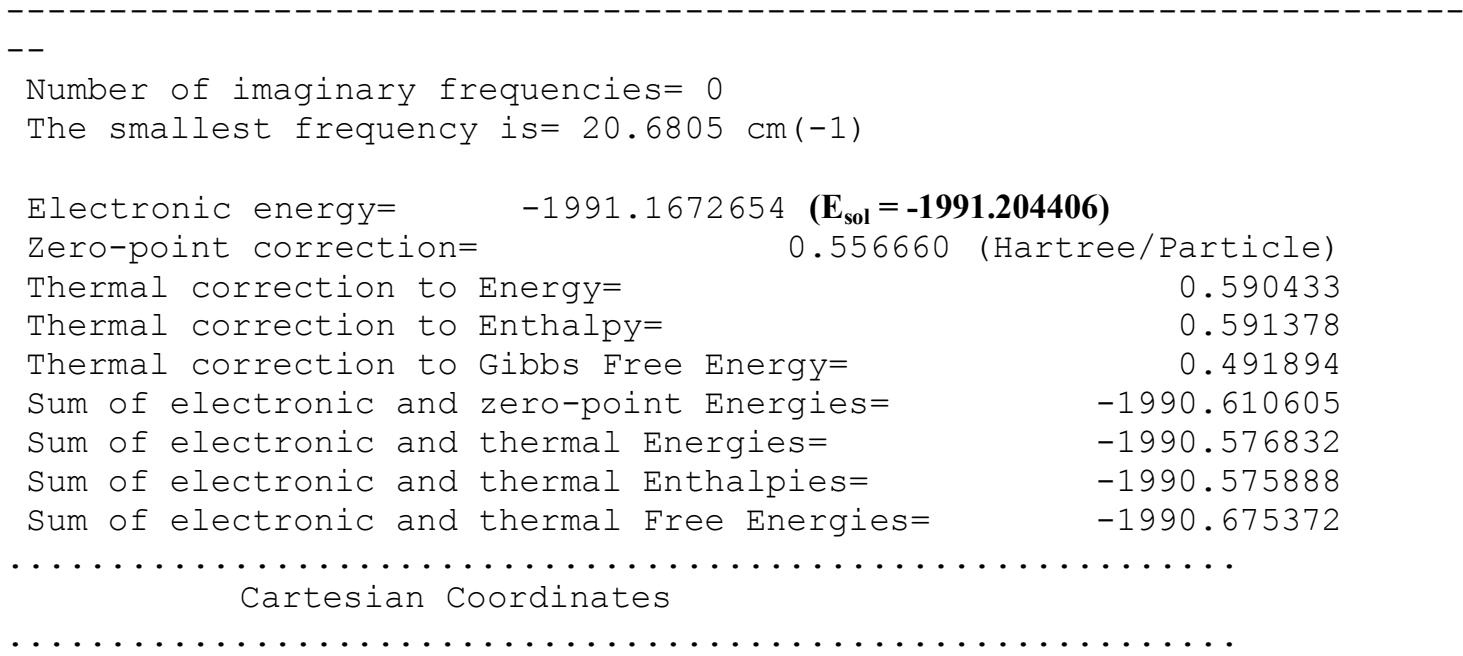




\begin{tabular}{|c|c|c|c|}
\hline 6 & -2.487206 & -3.133224 & 1.480373 \\
\hline 6 & -1.228873 & -2.716542 & 1.144643 \\
\hline 6 & -1.077806 & -1.548490 & 0.371095 \\
\hline 6 & -2.141766 & -0.782250 & -0.053378 \\
\hline 6 & -3.464882 & -1.249616 & 0.239265 \\
\hline 1 & -2.628175 & -4.026753 & 2.081405 \\
\hline 6 & -2.021964 & 2.906047 & -1.151894 \\
\hline 6 & -1.234936 & 2.772213 & -2.326673 \\
\hline 6 & -0.745302 & 1.553005 & -2.702590 \\
\hline 6 & -1.066912 & 0.417132 & -1.931803 \\
\hline 6 & -1.874409 & 0.465493 & -0.816366 \\
\hline 6 & -2.337248 & 1.752072 & -0.378516 \\
\hline 1 & -1.001054 & 3.661048 & -2.905185 \\
\hline 6 & -3.446885 & 3.179901 & 1.244086 \\
\hline 6 & -3.045920 & 1.931466 & 0.840481 \\
\hline 1 & -3.980099 & 3.294991 & 2.182219 \\
\hline 1 & -3.258616 & 1.068091 & 1.460778 \\
\hline 6 & -3.165364 & 4.319378 & 0.455395 \\
\hline 6 & -2.465082 & 4.181243 & -0.713943 \\
\hline 1 & -3.494403 & 5.299195 & 0.785167 \\
\hline 1 & -2.223581 & 5.048916 & -1.321585 \\
\hline 6 & -5.878875 & -1.070205 & 0.046422 \\
\hline 6 & -4.627142 & -0.598355 & -0.255418 \\
\hline 1 & -6.753846 & -0.562422 & -0.346201 \\
\hline 1 & -4.512998 & 0.277136 & -0.885505 \\
\hline 6 & -6.041987 & -2.217423 & 0.857510 \\
\hline 6 & -4.940287 & -2.878847 & 1.331782 \\
\hline 1 & -7.038894 & -2.576232 & 1.091109 \\
\hline 1 & -5.048380 & -3.771808 & 1.941310 \\
\hline 8 & -0.512977 & -0.796665 & -2.326390 \\
\hline 8 & 0.218099 & -1.143286 & 0.074485 \\
\hline 15 & 0.743343 & -1.310261 & -1.456887 \\
\hline 8 & 1.785097 & -0.135701 & -1.569143 \\
\hline 8 & 1.157636 & -2.672856 & -1.811804 \\
\hline 1 & 2.357668 & -2.688035 & 0.057817 \\
\hline 8 & 3.106082 & -2.115661 & 0.275915 \\
\hline 6 & 4.129248 & -2.421547 & -0.662856 \\
\hline 6 & 5.331091 & -1.549062 & -0.353724 \\
\hline 1 & 6.115394 & -1.758354 & -1.089797 \\
\hline 1 & 5.729418 & -1.791506 & 0.637871 \\
\hline 6 & 4.996715 & -0.053918 & -0.392089 \\
\hline 1 & 4.383668 & 0.163051 & -1.273666 \\
\hline 6 & 4.227394 & 0.338961 & 0.857105 \\
\hline 1 & 2.226523 & 0.128502 & -0.722403 \\
\hline 8 & 3.043226 & 0.931400 & 0.559454 \\
\hline 6 & 2.211592 & 1.527004 & 1.618239 \\
\hline 6 & 1.772929 & 0.447579 & 2.601454 \\
\hline 1 & 1.005039 & 0.861263 & 3.262097 \\
\hline 1 & 1.346915 & -0.397280 & 2.055015 \\
\hline 1 & 2.612859 & 0.098731 & 3.201457 \\
\hline 1 & 4.411227 & -3.480581 & -0.596762 \\
\hline 1 & 3.770311 & -2.231027 & -1.683871 \\
\hline 6 & 1.020464 & 2.087760 & 0.848919 \\
\hline 1 & 0.348900 & 2.613033 & 1.534107 \\
\hline 1 & 1.354509 & 2.790195 & 0.079667 \\
\hline 1 & 0.454415 & 1.285175 & 0.369976 \\
\hline 6 & 2.993424 & 2.650554 & 2.290703 \\
\hline 1 & 3.340588 & 3.368645 & 1.541762 \\
\hline
\end{tabular}




$\begin{array}{rrrr}1 & 2.331661 & 3.174935 & 2.985823 \\ 1 & 3.848819 & 2.261890 & 2.843259 \\ 6 & -3.630598 & -2.423334 & 1.028555 \\ 1 & -0.338165 & -3.251790 & 1.454283 \\ 1 & -0.104744 & 1.422904 & -3.567299 \\ 8 & 4.658234 & 0.211794 & 1.973443 \\ 6 & 6.270890 & 0.799101 & -0.423459 \\ 1 & 6.036455 & 1.867295 & -0.427264 \\ 1 & 6.878921 & 0.584073 & 0.459033 \\ 1 & 6.854526 & 0.571872 & -1.318877\end{array}$

$\mathrm{TS}$ with catalyst $(\mathrm{Ar}=$ phenylanthryl $)$

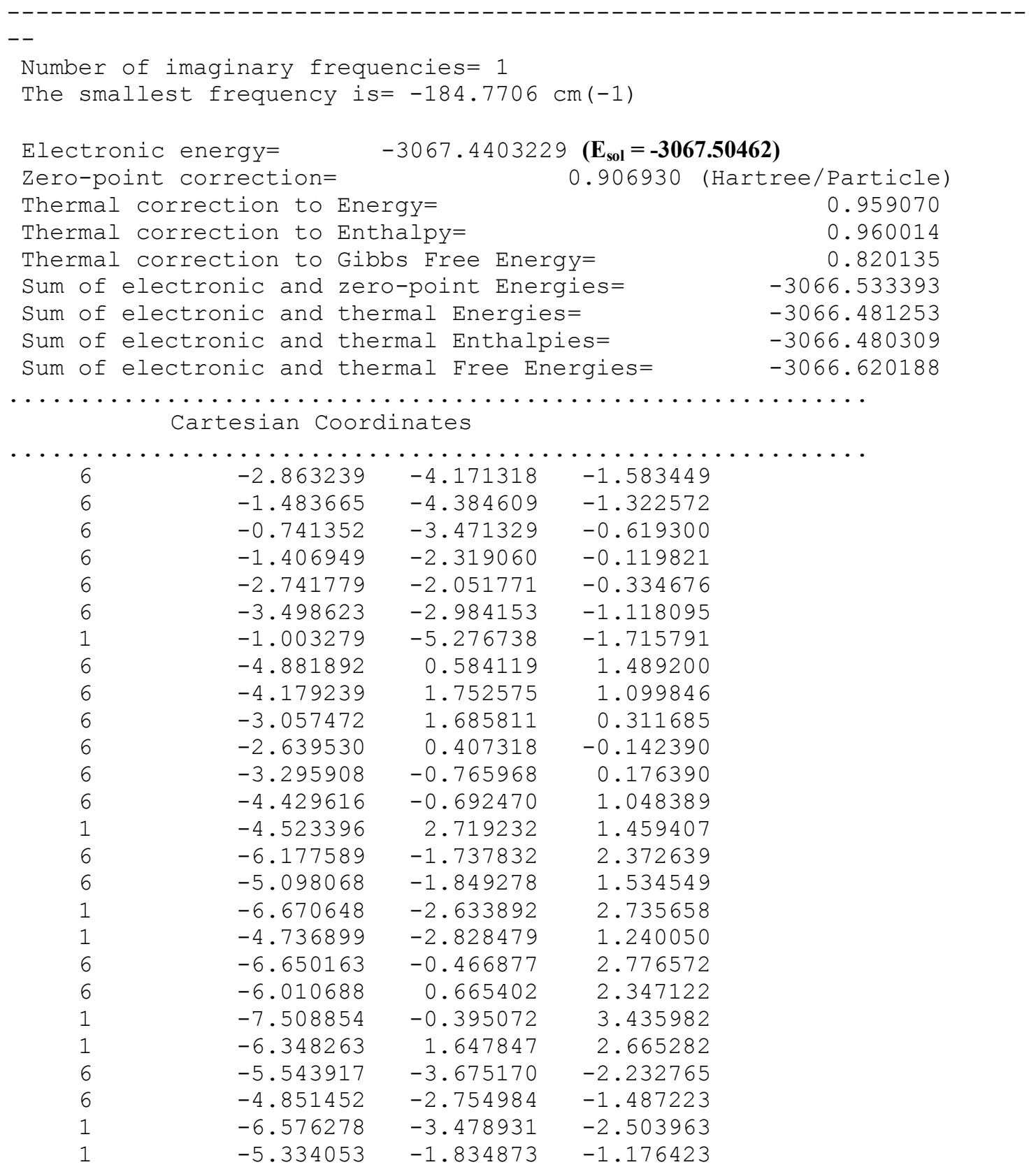




\begin{tabular}{|c|c|c|c|}
\hline 6 & -4.923721 & -4.872560 & -2.658006 \\
\hline 6 & -3.610741 & -5.107284 & -2.344634 \\
\hline 1 & -5.485025 & -5.594136 & -3.242468 \\
\hline 1 & -3.113447 & -6.012559 & -2.682140 \\
\hline 8 & -1.505912 & 0.351681 & -0.931375 \\
\hline 8 & -0.650208 & -1.423891 & 0.608182 \\
\hline 15 & -0.139729 & -0.086251 & -0.159553 \\
\hline 8 & 0.234082 & 0.889050 & 0.923481 \\
\hline 8 & 0.871216 & -0.380246 & -1.238620 \\
\hline 1 & 1.348509 & 1.712784 & 0.600208 \\
\hline 6 & -2.283239 & 2.903842 & -0.066885 \\
\hline 6 & -1.484222 & 3.606585 & 0.919395 \\
\hline 6 & -2.320554 & 3.348650 & -1.349610 \\
\hline 6 & -0.772261 & 4.775506 & 0.543129 \\
\hline 6 & -1.616472 & 4.526142 & -1.767984 \\
\hline 6 & -0.851411 & 5.258497 & -0.829185 \\
\hline 6 & 0.738043 & -3.557401 & -0.472491 \\
\hline 6 & 1.505275 & -3.397024 & -1.580999 \\
\hline 6 & 1.363601 & -3.693714 & 0.826589 \\
\hline 6 & 2.933712 & -3.296961 & -1.507274 \\
\hline 6 & 2.774791 & -3.619519 & 0.939041 \\
\hline 6 & 3.581204 & -3.391977 & -0.253041 \\
\hline 8 & 2.128108 & 2.293086 & 0.201497 \\
\hline 6 & 1.656681 & 2.913265 & -0.999744 \\
\hline 6 & 2.381836 & 2.231842 & -2.161490 \\
\hline 1 & 2.485648 & 2.901921 & -3.018503 \\
\hline 1 & 1.829216 & 1.343522 & -2.479672 \\
\hline 6 & 3.746876 & 1.826122 & -1.611036 \\
\hline 6 & 3.528782 & 1.205062 & -0.238439 \\
\hline 1 & 2.194055 & -0.192349 & -0.711941 \\
\hline 8 & 3.047096 & -0.001491 & -0.131633 \\
\hline 1 & 0.568543 & 2.795818 & -1.061623 \\
\hline 1 & 1.888438 & 3.981555 & -0.940958 \\
\hline 8 & 4.470103 & 1.544045 & 0.623051 \\
\hline 6 & 4.540660 & 0.971010 & 1.972223 \\
\hline 6 & 3.193510 & 1.070113 & 2.679740 \\
\hline 1 & 2.438603 & 0.431535 & 2.216925 \\
\hline 1 & 3.324609 & 0.745515 & 3.716189 \\
\hline 1 & 2.833204 & 2.102260 & 2.679144 \\
\hline 6 & 5.570899 & 1.870484 & 2.645374 \\
\hline 1 & 5.728668 & 1.547172 & 3.677364 \\
\hline 1 & 6.524563 & 1.821859 & 2.112986 \\
\hline 1 & 5.224200 & 2.906670 & 2.648890 \\
\hline 6 & 5.049382 & -0.465729 & 1.898026 \\
\hline 1 & 5.930978 & -0.517671 & 1.251282 \\
\hline 1 & 5.334488 & -0.795791 & 2.902138 \\
\hline 1 & 4.285123 & -1.137751 & 1.508633 \\
\hline 6 & 4.504834 & 0.838174 & -2.495483 \\
\hline 1 & 4.729028 & 1.293826 & -3.463041 \\
\hline 1 & 5.445138 & 0.534781 & -2.026078 \\
\hline 1 & 3.907661 & -0.062736 & -2.662704 \\
\hline 1 & 4.351981 & 2.722166 & -1.433982 \\
\hline 1 & 1.028298 & -3.253866 & -2.546697 \\
\hline 1 & -2.898659 & 2.803663 & -2.091616 \\
\hline 6 & 0.025302 & 5.416717 & 1.516250 \\
\hline 1 & 0.585187 & 6.307208 & 1.255448 \\
\hline 6 & -1.364480 & 3.120295 & 2.240172 \\
\hline 7 & -1.875893 & 2.203215 & 2.508520 \\
\hline
\end{tabular}




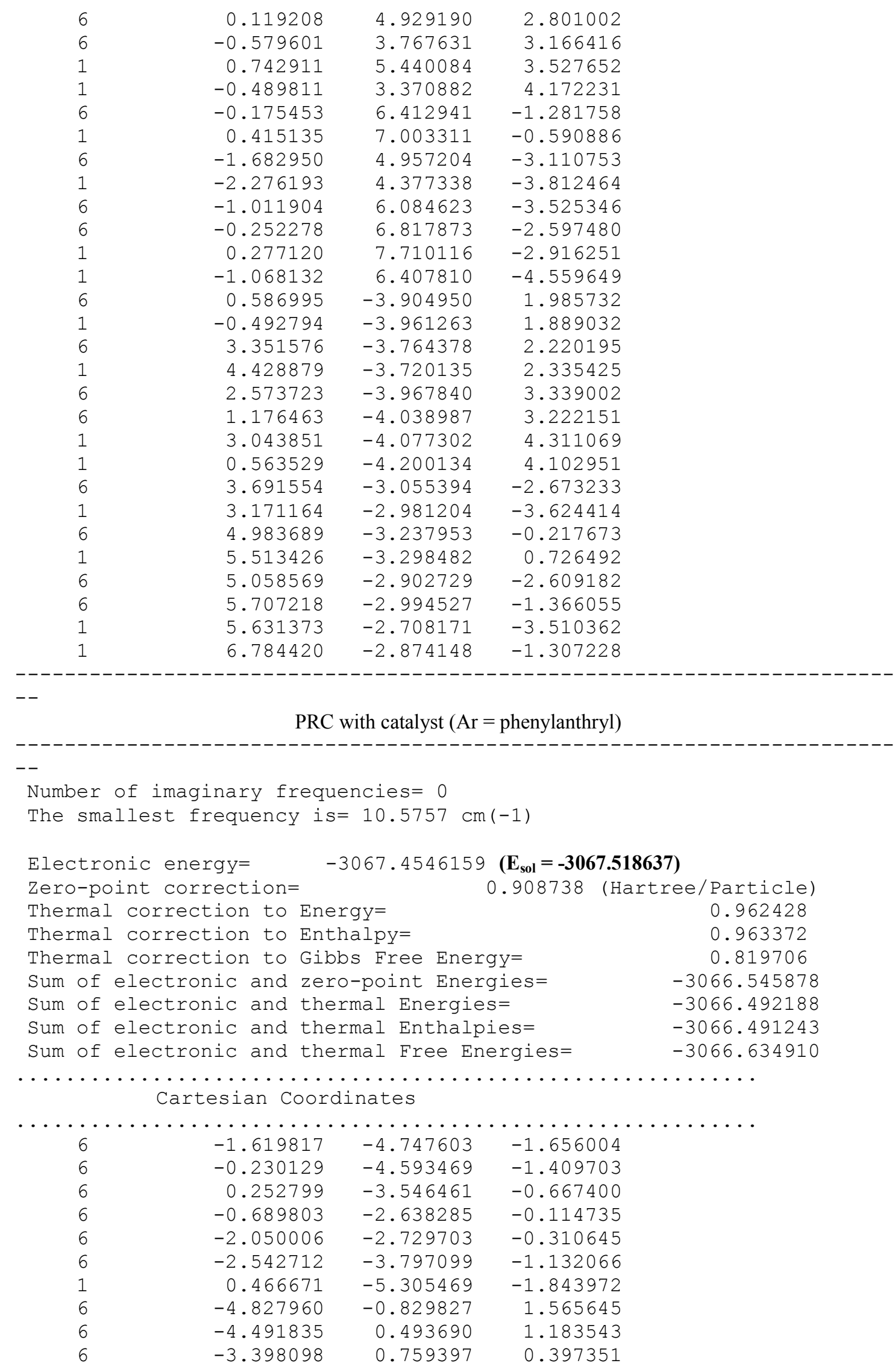




\begin{tabular}{|c|c|c|c|}
\hline 6 & -2.627551 & -0.342993 & -0.052244 \\
\hline 6 & -2.925797 & -1.658416 & 0.242753 \\
\hline 6 & -4.033435 & -1.920618 & 1.111276 \\
\hline 1 & -5.103638 & 1.318500 & 1.540252 \\
\hline 6 & -5.414334 & -3.436953 & 2.411187 \\
\hline 6 & -4.347744 & -3.226781 & 1.575562 \\
\hline 1 & -5.635161 & -4.440866 & 2.759110 \\
\hline 1 & -3.725767 & -4.059799 & 1.266284 \\
\hline 6 & -6.225978 & -2.356674 & 2.831365 \\
\hline 6 & -5.933489 & -1.083542 & 2.420305 \\
\hline 1 & -7.069589 & -2.539977 & 3.488669 \\
\hline 1 & -6.536137 & -0.241426 & 2.748862 \\
\hline 6 & -4.344727 & -4.958154 & -2.274570 \\
\hline 6 & -3.913024 & -3.922363 & -1.485616 \\
\hline 1 & -5.395610 & -5.033306 & -2.534635 \\
\hline 1 & -4.620491 & -3.181727 & -1.130317 \\
\hline 6 & -3.432927 & -5.923732 & -2.760164 \\
\hline 6 & -2.100469 & -5.813185 & -2.460808 \\
\hline 1 & -3.788790 & -6.740443 & -3.379522 \\
\hline 1 & -1.384324 & -6.534576 & -2.844322 \\
\hline 8 & -1.507646 & -0.065689 & -0.825695 \\
\hline 8 & -0.186372 & -1.599914 & 0.650171 \\
\hline 15 & -0.110306 & -0.129630 & -0.016024 \\
\hline 8 & 0.076559 & 0.881777 & 1.042273 \\
\hline 8 & 0.912169 & -0.163465 & -1.190923 \\
\hline 1 & 0.715458 & 2.535974 & 0.701966 \\
\hline 6 & -3.035304 & 2.152380 & 0.000935 \\
\hline 6 & -2.417682 & 3.056608 & 0.951291 \\
\hline 6 & -3.304014 & 2.572531 & -1.262214 \\
\hline 6 & -2.134233 & 4.392948 & 0.564476 \\
\hline 6 & -3.037531 & 3.915693 & -1.688754 \\
\hline 6 & -2.473547 & 4.841564 & -0.779302 \\
\hline 6 & 1.705326 & -3.246629 & -0.535258 \\
\hline 6 & 2.384040 & -2.853502 & -1.643403 \\
\hline 6 & 2.372101 & -3.275698 & 0.749560 \\
\hline 6 & 3.743698 & -2.402741 & -1.580734 \\
\hline 6 & 3.723889 & -2.858534 & 0.849432 \\
\hline 6 & 4.422854 & -2.389522 & -0.340251 \\
\hline 8 & 1.170393 & 3.332924 & 0.372992 \\
\hline 6 & 0.753668 & 3.589360 & -0.950463 \\
\hline 6 & 1.680070 & 2.942386 & -1.990454 \\
\hline 1 & 1.588602 & 3.478830 & -2.941868 \\
\hline 1 & 1.378018 & 1.909929 & -2.189329 \\
\hline 6 & 3.148028 & 2.954402 & -1.556283 \\
\hline 6 & 3.338639 & 2.105873 & -0.317850 \\
\hline 1 & 1.799834 & 0.269590 & -0.907521 \\
\hline 8 & 2.936389 & 0.947398 & -0.222919 \\
\hline 1 & -0.277562 & 3.249376 & -1.118413 \\
\hline 1 & 0.757538 & 4.678951 & -1.082206 \\
\hline 8 & 4.086289 & 2.685616 & 0.593961 \\
\hline 6 & 4.277391 & 2.092830 & 1.922575 \\
\hline 6 & 2.925722 & 1.971193 & 2.617017 \\
\hline 1 & 2.283327 & 1.233092 & 2.133115 \\
\hline 1 & 3.090657 & 1.664500 & 3.654431 \\
\hline 1 & 2.411892 & 2.936336 & 2.607805 \\
\hline 6 & 5.154679 & 3.128214 & 2.615543 \\
\hline 1 & 5.358199 & 2.812915 & 3.642067 \\
\hline 7 & 6.105765 & 3.240511 & 2.087896 \\
\hline
\end{tabular}




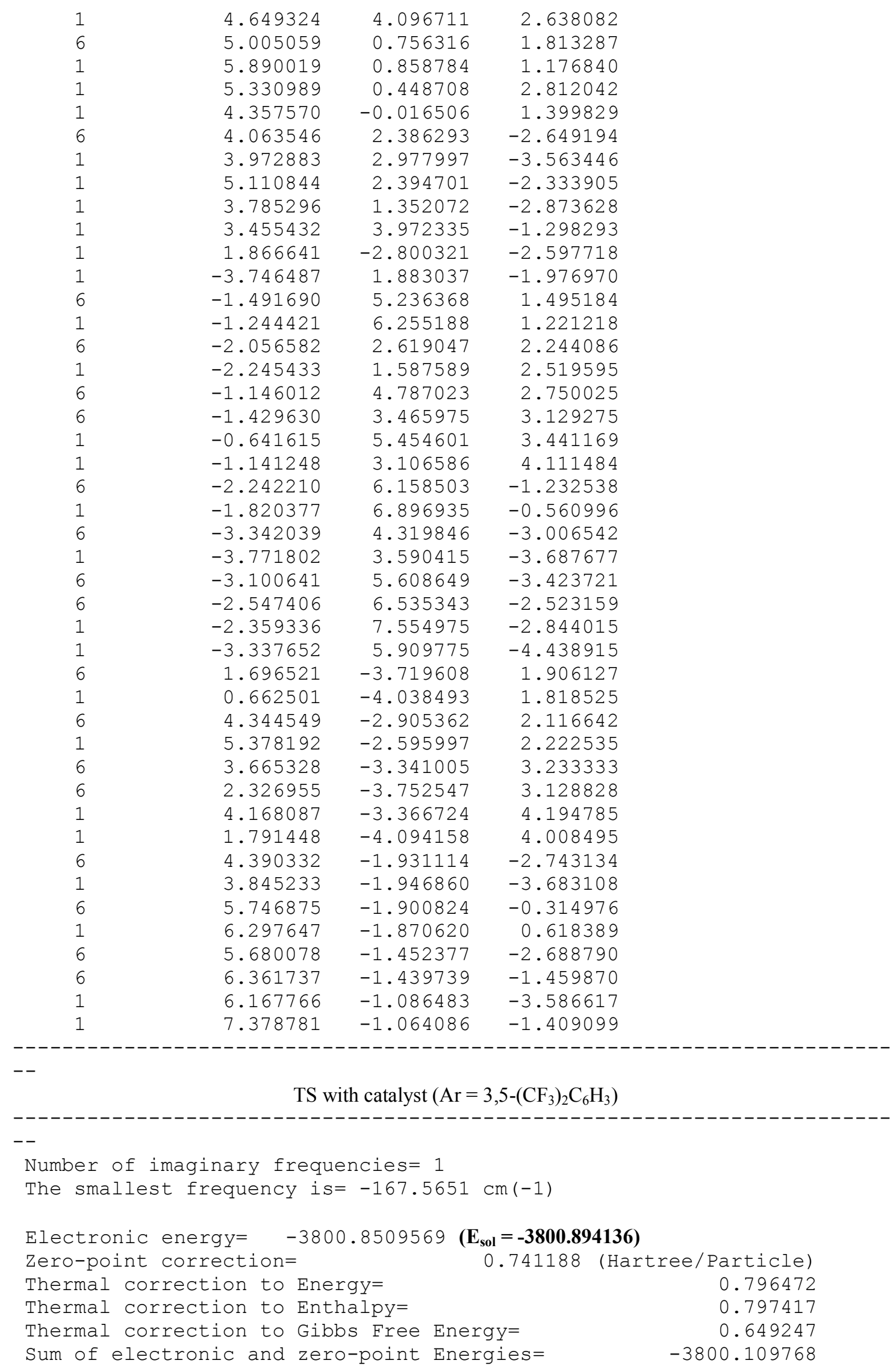


$\begin{array}{ll}\text { Sum of electronic and thermal Energies= } & -3800.054484 \\ \text { Sum of electronic and thermal Enthalpies= } & -3800.053540 \\ \text { Sum of electronic and thermal Eree Energies= } & -3800.201710\end{array}$

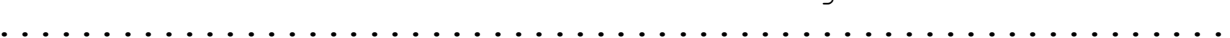

Cartesian Coordinates

\begin{tabular}{|c|c|c|c|}
\hline 6 & 1.235706 & 4.914825 & 1.203702 \\
\hline 6 & 2.267039 & 4.000176 & 0.873844 \\
\hline 6 & 2.005454 & 2.837335 & 0.191603 \\
\hline 6 & 0.667267 & 2.593372 & -0.214973 \\
\hline 6 & -0.378537 & 3.442064 & 0.075204 \\
\hline 6 & -0.108231 & 4.627555 & 0.831567 \\
\hline 1 & 3.285180 & 4.222497 & 1.183974 \\
\hline 6 & -3.868001 & 3.348654 & -1.544721 \\
\hline 6 & -4.282976 & 2.067393 & -1.105132 \\
\hline 6 & -3.468765 & 1.269114 & -0.337828 \\
\hline 6 & -2.197748 & 1.780290 & 0.039556 \\
\hline 6 & -1.748127 & 3.028971 & -0.337200 \\
\hline 6 & -2.580676 & 3.833023 & -1.180267 \\
\hline 1 & -5.268857 & 1.709421 & -1.391525 \\
\hline 6 & -2.981485 & 5.822356 & -2.512937 \\
\hline 6 & -2.154879 & 5.082468 & -1.706875 \\
\hline 1 & -2.636077 & 6.772094 & -2.908114 \\
\hline 1 & -1.160262 & 5.444169 & -1.470998 \\
\hline 6 & -4.276243 & 5.357396 & -2.841788 \\
\hline 6 & -4.704917 & 4.144696 & -2.371203 \\
\hline 1 & -4.919841 & 5.957298 & -3.476532 \\
\hline 1 & -5.689053 & 3.764952 & -2.631230 \\
\hline 6 & -0.829537 & 6.638133 & 1.985706 \\
\hline 6 & -1.132855 & 5.511019 & 1.265315 \\
\hline 1 & -1.626639 & 7.298976 & 2.310427 \\
\hline 1 & -2.165297 & 5.281184 & 1.025783 \\
\hline 6 & 0.511231 & 6.944465 & 2.317341 \\
\hline 6 & 1.517832 & 6.096015 & 1.939046 \\
\hline 1 & 0.736432 & 7.842813 & 2.882321 \\
\hline 1 & 2.550144 & 6.306828 & 2.204040 \\
\hline 8 & -1.380153 & 0.972038 & 0.807209 \\
\hline 8 & 0.402123 & 1.417633 & -0.893463 \\
\hline 15 & -0.157216 & 0.207422 & 0.043817 \\
\hline 8 & -0.667561 & -0.860279 & -0.884574 \\
\hline 8 & 0.840758 & -0.167522 & 1.106368 \\
\hline 1 & -0.252104 & -2.178289 & -0.576128 \\
\hline 6 & -3.885320 & -0.099449 & 0.056859 \\
\hline 6 & -4.448291 & -0.950942 & -0.895873 \\
\hline 6 & -3.749075 & -0.555199 & 1.373189 \\
\hline 6 & -4.884592 & -2.222227 & -0.536011 \\
\hline 6 & -4.191928 & -1.826435 & 1.717926 \\
\hline 6 & -4.767315 & -2.670573 & 0.772641 \\
\hline 6 & 3.062485 & 1.825620 & -0.056174 \\
\hline 6 & 3.867968 & 1.403650 & 1.005140 \\
\hline 6 & 3.275710 & 1.277982 & -1.322409 \\
\hline 6 & 4.874390 & 0.469326 & 0.795319 \\
\hline 6 & 4.297060 & 0.352967 & -1.520900 \\
\hline 6 & 5.107354 & -0.057872 & -0.470398 \\
\hline 8 & -0.023749 & -3.137050 & -0.198849 \\
\hline 6 & -0.879924 & -3.376310 & 0.926338 \\
\hline 6 & -0.045810 & -3.064429 & 2.163454 \\
\hline 1 & -0.436402 & -3.561596 & 3.053148 \\
\hline
\end{tabular}




\begin{tabular}{|c|c|c|c|}
\hline 1 & -0.027956 & -1.985808 & 2.347277 \\
\hline 6 & 1.360217 & -3.559168 & 1.823095 \\
\hline 6 & 1.658061 & -3.206981 & 0.364426 \\
\hline 1 & 1.693298 & -1.259590 & 0.592689 \\
\hline 8 & 2.149744 & -2.024734 & 0.069273 \\
\hline 1 & -1.761759 & -2.737829 & 0.827354 \\
\hline 1 & -1.187884 & -4.425409 & 0.895319 \\
\hline 8 & 2.166372 & -4.234303 & -0.297501 \\
\hline 6 & 2.679109 & -4.112077 & -1.668505 \\
\hline 6 & 1.714056 & -3.339418 & -2.561878 \\
\hline 1 & 1.615911 & -2.297975 & -2.253565 \\
\hline 1 & 2.106398 & -3.353026 & -3.582508 \\
\hline 1 & 0.728999 & -3.811129 & -2.557956 \\
\hline 6 & 2.766722 & -5.566689 & -2.113988 \\
\hline 1 & 3.179337 & -5.620253 & -3.124571 \\
\hline 1 & 3.414557 & -6.133177 & -1.440084 \\
\hline 1 & 1.774412 & -6.024458 & -2.113435 \\
\hline 6 & 4.065585 & -3.480661 & -1.622966 \\
\hline 1 & 4.722930 & -4.067617 & -0.974796 \\
\hline 1 & 4.492377 & -3.460054 & -2.629328 \\
\hline 1 & 4.010752 & -2.461397 & -1.244562 \\
\hline 6 & 2.448809 & -2.988670 & 2.729323 \\
\hline 1 & 2.287165 & -3.320192 & 3.757664 \\
\hline 1 & 3.441541 & -3.316029 & 2.410283 \\
\hline 1 & 2.428854 & -1.895550 & 2.714197 \\
\hline 1 & 1.373041 & -4.653841 & 1.859520 \\
\hline 1 & 3.691390 & 1.795473 & 2.001776 \\
\hline 1 & 2.651188 & 1.581223 & -2.156813 \\
\hline 1 & 5.903646 & -0.775155 & -0.633093 \\
\hline 1 & -5.120591 & -3.657295 & 1.053936 \\
\hline 1 & -3.304495 & 0.085189 & 2.126558 \\
\hline 1 & -4.532657 & -0.623676 & -1.927029 \\
\hline 6 & 5.680083 & -0.025800 & 1.962332 \\
\hline 6 & 4.515568 & -0.180448 & -2.909142 \\
\hline 6 & -3.993734 & -2.345197 & 3.113066 \\
\hline 6 & -5.431017 & -3.155065 & -1.580928 \\
\hline 9 & -6.402611 & -3.933141 & -1.082335 \\
\hline 9 & -5.050462 & -3.051755 & 3.534444 \\
\hline 9 & -3.775679 & -1.364067 & 3.993370 \\
\hline 9 & -2.931125 & -3.174623 & 3.170694 \\
\hline 9 & -4.477737 & -3.971531 & -2.051533 \\
\hline 9 & -5.936591 & -2.489165 & -2.626985 \\
\hline 9 & 5.102896 & -1.090592 & 2.543490 \\
\hline 9 & 5.810191 & 0.913413 & 2.909307 \\
\hline 9 & 6.910705 & -0.404030 & 1.588856 \\
\hline 9 & 5.579598 & -0.993113 & -2.975556 \\
\hline 9 & 3.453470 & -0.876655 & -3.345542 \\
\hline 9 & 4.710004 & 0.813935 & -3.787357 \\
\hline
\end{tabular}

PRC with catalyst $\left(\mathrm{Ar}=3,5-\left(\mathrm{CF}_{3}\right)_{2} \mathrm{C}_{6} \mathrm{H}_{3}\right)$

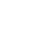

Number of imaginary frequencies $=0$

The smallest frequency is $=11.0838 \mathrm{~cm}(-1)$

Electronic energy=

Zero-point correction=
$-3800.8652435\left(\mathbf{E}_{\mathrm{sol}}=\mathbf{- 3 8 0 0 . 9 0 8 6 9 2}\right)$

0.741970 (Hartree/Particle) 


$\begin{array}{lr}\text { Thermal correction to Energy= } & 0.798853 \\ \text { Thermal correction to Enthalpy= } & 0.799797 \\ \text { Thermal correction to Gibs Free Energy= } & 0.647498 \\ \text { Sum of electronic and zero-point Energies= } & -3800.123274 \\ \text { Sum of electronic and thermal Energies= } & -3800.066390 \\ \text { Sum of electronic and thermal Enthalpies= } & -3800.065446 \\ \text { Sum of electronic and thermal Free Energies } & -3800.217746\end{array}$

Cartesian Coordinates

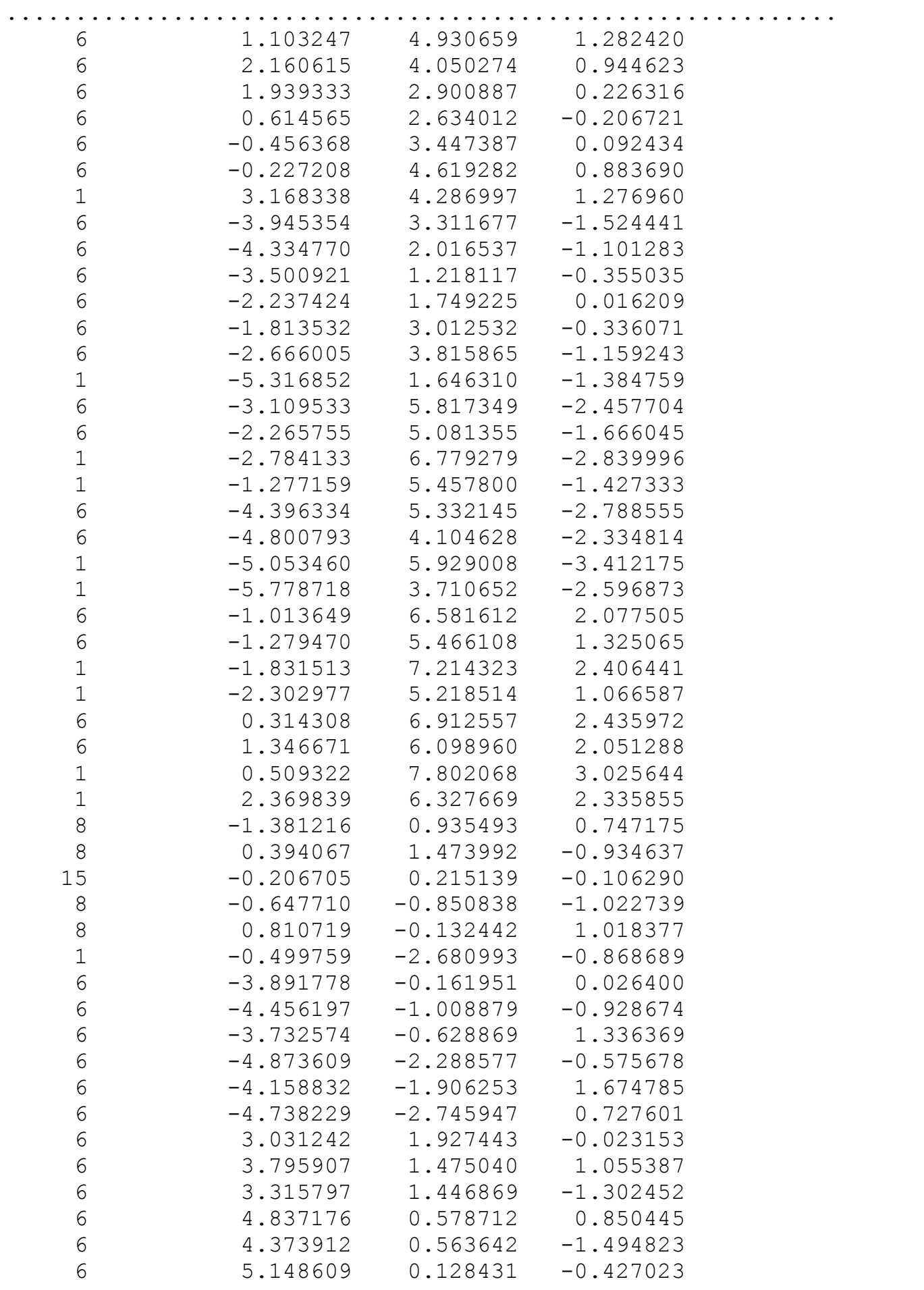




\begin{tabular}{|c|c|c|c|}
\hline 8 & -0.325092 & -3.611726 & -0.640321 \\
\hline 6 & -0.921278 & -3.891925 & 0.605423 \\
\hline 6 & -0.144893 & -3.269005 & 1.761960 \\
\hline 1 & -0.574832 & -3.595462 & 2.714282 \\
\hline 1 & -0.240299 & -2.177437 & 1.734910 \\
\hline 6 & 1.344278 & -3.646952 & 1.752616 \\
\hline 6 & 2.015124 & -3.212096 & 0.466716 \\
\hline 1 & 1.414621 & -0.936103 & 0.743730 \\
\hline 8 & 2.165507 & -2.026033 & 0.157806 \\
\hline 1 & -1.966439 & -3.554782 & 0.638885 \\
\hline 1 & -0.924975 & -4.983040 & 0.711505 \\
\hline 8 & 2.512319 & -4.206655 & -0.223918 \\
\hline 6 & 3.119965 & -4.014565 & -1.550379 \\
\hline 6 & 2.115313 & -3.370244 & -2.498191 \\
\hline 1 & 1.866675 & -2.353251 & -2.193303 \\
\hline 1 & 2.558991 & -3.332189 & -3.497306 \\
\hline 1 & 1.198396 & -3.961679 & -2.528698 \\
\hline 6 & 3.428622 & -5.442625 & -1.979488 \\
\hline 1 & 3.895858 & -5.435566 & -2.967542 \\
\hline 1 & 4.112984 & -5.916853 & -1.270981 \\
\hline 1 & 2.508955 & -6.030272 & -2.028623 \\
\hline 6 & 4.399874 & -3.202475 & -1.404722 \\
\hline 1 & 5.070956 & -3.675816 & -0.681314 \\
\hline 1 & 4.908514 & -3.150853 & -2.371216 \\
\hline 1 & 4.171226 & -2.190496 & -1.074411 \\
\hline 6 & 2.085480 & -2.997582 & 2.928278 \\
\hline 1 & 1.655795 & -3.340647 & 3.872349 \\
\hline 1 & 3.149689 & -3.246623 & 2.921818 \\
\hline 1 & 1.991244 & -1.908494 & 2.885180 \\
\hline 1 & 1.436890 & -4.736294 & 1.804790 \\
\hline 1 & 3.559083 & 1.810860 & 2.060129 \\
\hline 1 & 2.718603 & 1.769590 & -2.149715 \\
\hline 1 & 5.972641 & -0.557950 & -0.585395 \\
\hline 1 & -5.078053 & -3.739364 & 1.003075 \\
\hline 1 & -3.289100 & 0.009252 & 2.092191 \\
\hline 1 & -4.557305 & -0.672885 & -1.955477 \\
\hline 6 & 5.578713 & 0.024106 & 2.033031 \\
\hline 6 & 4.648617 & 0.065825 & -2.886603 \\
\hline 6 & -3.975076 & -2.430654 & 3.070486 \\
\hline 6 & -5.422168 & -3.219322 & -1.621546 \\
\hline 9 & -6.396850 & -3.993764 & -1.122235 \\
\hline 9 & -5.088792 & -3.024471 & 3.521028 \\
\hline 9 & -3.646188 & -1.465823 & 3.935235 \\
\hline 9 & -3.001921 & -3.359650 & 3.116401 \\
\hline 9 & -4.472672 & -4.037121 & -2.092586 \\
\hline 9 & -5.927865 & -2.549680 & -2.665958 \\
\hline 9 & 4.963693 & -1.063819 & 2.524989 \\
\hline 9 & 5.657844 & 0.913999 & 3.031879 \\
\hline 9 & 6.826455 & -0.341907 & 1.710185 \\
\hline 9 & 5.787281 & -0.638858 & -2.951954 \\
\hline 9 & 3.661029 & -0.729870 & -3.325806 \\
\hline 9 & 4.746328 & 1.079122 & -3.758330 \\
\hline
\end{tabular}

TS with catalyst $\left(\mathrm{Ar}=2,4,6-\left({ }^{i} \operatorname{Pr}\right)_{3} \mathrm{C}_{6} \mathrm{H}_{2}\right)$ with phenyl and methyl groups at the chiral carbon of substrate --

Number of imaginary frequencies $=1$ 


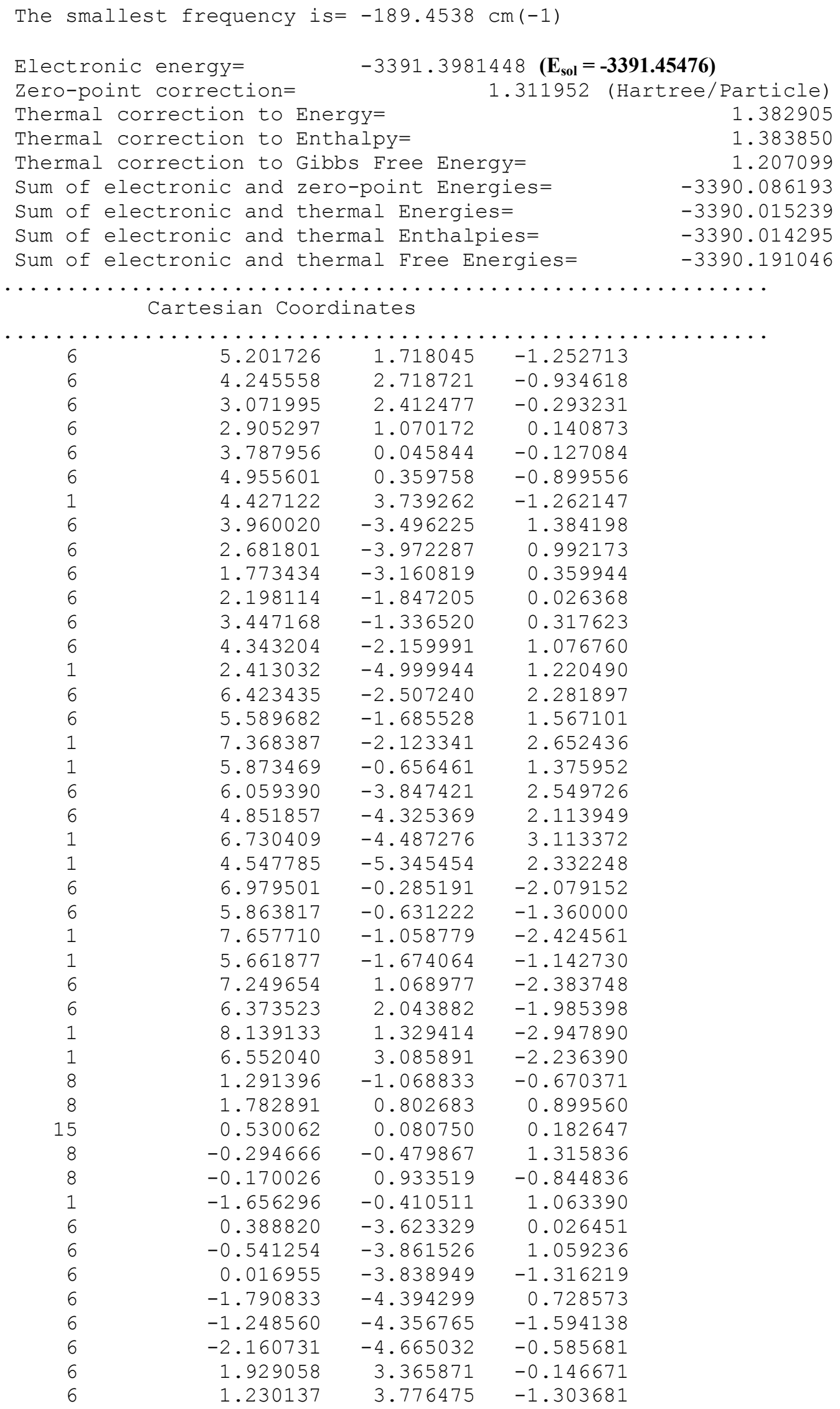




\begin{tabular}{|c|c|c|c|}
\hline 6 & 1.483524 & 3.772457 & 1.124115 \\
\hline 6 & 0.123060 & 4.611188 & -1.158767 \\
\hline 6 & 0.363334 & 4.600753 & 1.214839 \\
\hline 6 & -0.334274 & 5.028104 & 0.089397 \\
\hline 8 & -2.658053 & -0.433505 & 0.709134 \\
\hline 1 & 0.019493 & 4.922414 & 2.196454 \\
\hline 1 & -0.410479 & 4.930759 & -2.050864 \\
\hline 1 & -1.521136 & -4.540635 & -2.631873 \\
\hline 1 & -2.503963 & -4.599391 & 1.523841 \\
\hline 6 & 2.186959 & 3.365454 & 2.409714 \\
\hline 1 & 2.984647 & 2.659433 & 2.159482 \\
\hline 6 & 1.616792 & 3.332627 & -2.708579 \\
\hline 1 & 2.330923 & 2.509174 & -2.624719 \\
\hline 6 & -1.544915 & 5.932236 & 0.216921 \\
\hline 1 & -1.820420 & 5.957417 & 1.279857 \\
\hline 6 & 1.233907 & 2.660137 & 3.381948 \\
\hline 1 & 0.800751 & 1.766470 & 2.925229 \\
\hline 1 & 1.773448 & 2.357349 & 4.284908 \\
\hline 1 & 0.418396 & 3.323798 & 3.691118 \\
\hline 6 & 2.841917 & 4.585135 & 3.070660 \\
\hline 1 & 3.538583 & 5.076230 & 2.385354 \\
\hline 1 & 2.086776 & 5.320716 & 3.367111 \\
\hline 1 & 3.391991 & 4.287369 & 3.968621 \\
\hline 6 & 0.418781 & 2.802511 & -3.505477 \\
\hline 1 & -0.303733 & 3.594942 & -3.728656 \\
\hline 1 & 0.762024 & 2.397841 & -4.462815 \\
\hline 1 & -0.085962 & 2.009693 & -2.949496 \\
\hline 6 & 2.295271 & 4.480374 & -3.467181 \\
\hline 1 & 2.603153 & 4.155675 & -4.465968 \\
\hline 1 & 1.602872 & 5.321638 & -3.582110 \\
\hline 1 & 3.178166 & 4.849419 & -2.937305 \\
\hline 6 & -2.745462 & 5.393380 & -0.567323 \\
\hline 1 & -2.566522 & 5.447026 & -1.646898 \\
\hline 1 & -2.939202 & 4.348628 & -0.309819 \\
\hline 1 & -3.641646 & 5.984747 & -0.352761 \\
\hline 6 & -1.204646 & 7.361746 & -0.220522 \\
\hline 1 & -2.069290 & 8.022706 & -0.103470 \\
\hline 1 & -0.375713 & 7.766887 & 0.366041 \\
\hline 1 & -0.907878 & 7.374713 & -1.274709 \\
\hline 6 & -0.238523 & -3.549407 & 2.518743 \\
\hline 1 & 0.614590 & -2.864867 & 2.543359 \\
\hline 6 & 0.944115 & -3.536233 & -2.483042 \\
\hline 1 & 1.878310 & -3.126592 & -2.089607 \\
\hline 6 & -3.502772 & -5.295721 & -0.911235 \\
\hline 1 & -4.036525 & -5.434665 & 0.037527 \\
\hline 6 & -4.367319 & -4.408851 & -1.815839 \\
\hline 1 & -5.289790 & -4.929487 & -2.090667 \\
\hline 1 & -4.649102 & -3.472705 & -1.324434 \\
\hline 1 & -3.836478 & -4.160682 & -2.74106 \\
\hline 6 & -3.306406 & -6.677861 & -1.54722 \\
\hline 1 & -2.810031 & -6.589337 & -2.519447 \\
\hline 1 & -2.688521 & -7.318274 & -0.912630 \\
\hline 1 & -4.270437 & -7.170321 & -1.70653 \\
\hline 6 & 0.331234 & -2.478762 & -3.40934 \\
\hline 1 & -0.590329 & -2.848442 & -3.87223 \\
\hline 1 & 0.101754 & -1.564082 & -2.855558 \\
\hline 1 & 1.029508 & -2.227222 & -4.21348 \\
\hline 6 & 1.294459 & -4.813149 & -3.25644 \\
\hline
\end{tabular}




\begin{tabular}{|c|c|c|c|}
\hline & & & \\
\hline 1 & 1.752610 & -5.557212 & -2.598712 \\
\hline 1 & 0.400843 & -5.262633 & -3.702025 \\
\hline 1 & 1.995592 & -4.589526 & -4.066246 \\
\hline 6 & 0.123862 & -4.821970 & 3.295687 \\
\hline 1 & 0.324670 & -4.586083 & 4.345386 \\
\hline 1 & -0.705883 & -5.536623 & 3.260872 \\
\hline 1 & 1.007321 & -5.315367 & 2.883609 \\
\hline 6 & -1.409488 & -2.841113 & 3.211803 \\
\hline 1 & -1.083551 & -2.450113 & 4.180194 \\
\hline 1 & -1.776357 & -2.007350 & 2.610652 \\
\hline 1 & -2.241218 & -3.528598 & 3.399568 \\
\hline 6 & -2.655367 & -1.228614 & -0.487088 \\
\hline 6 & -2.985106 & -0.278585 & -1.633873 \\
\hline 1 & -3.489335 & -0.795215 & -2.454944 \\
\hline 1 & -2.067235 & 0.176682 & -2.017273 \\
\hline 6 & -3.902376 & 0.814025 & -1.045088 \\
\hline 6 & -3.232554 & 1.230030 & 0.284927 \\
\hline 1 & -1.338055 & 1.558057 & -0.312478 \\
\hline 8 & -2.130485 & 1.935133 & 0.262201 \\
\hline 1 & -1.674698 & -1.704659 & -0.600936 \\
\hline 1 & -3.406764 & -2.010634 & -0.367341 \\
\hline 8 & -4.092525 & 1.555404 & 1.228771 \\
\hline 6 & -3.683009 & 2.063389 & 2.546245 \\
\hline 6 & -2.612184 & 1.173851 & 3.165915 \\
\hline 1 & -1.674985 & 1.213088 & 2.607093 \\
\hline 1 & -2.414634 & 1.527526 & 4.182103 \\
\hline 1 & -2.954155 & 0.136569 & 3.220511 \\
\hline 6 & -4.985730 & 1.971340 & 3.332033 \\
\hline 1 & -4.829476 & 2.325616 & 4.354062 \\
\hline 1 & -5.758458 & 2.583518 & 2.860111 \\
\hline 1 & -5.335395 & 0.936233 & 3.364472 \\
\hline 6 & -3.220832 & 3.512764 & 2.433684 \\
\hline 1 & -3.960864 & 4.103960 & 1.885716 \\
\hline 1 & -3.124552 & 3.930846 & 3.440677 \\
\hline 1 & -2.257271 & 3.583194 & 1.926179 \\
\hline 6 & -3.895703 & 2.051696 & -1.954185 \\
\hline 1 & -4.176995 & 1.777910 & -2.973073 \\
\hline 1 & -4.590927 & 2.813022 & -1.587912 \\
\hline 1 & -2.892437 & 2.482264 & -1.993024 \\
\hline 6 & -5.308186 & 0.233451 & -0.841742 \\
\hline 6 & -6.306686 & 0.469086 & -1.791001 \\
\hline 6 & -5.617236 & -0.621068 & 0.228145 \\
\hline 6 & -7.562968 & -0.125007 & -1.686544 \\
\hline 1 & -6.117662 & 1.126708 & -2.631788 \\
\hline 6 & -6.870281 & -1.214972 & 0.332332 \\
\hline 1 & -4.878613 & -0.810895 & 0.999148 \\
\hline 6 & -7.851335 & -0.973178 & -0.625323 \\
\hline 1 & -8.315154 & 0.080608 & -2.441705 \\
\hline 1 & -7.080665 & -1.867941 & 1.173833 \\
\hline 1 & -8.828849 & -1.436836 & -0.540325 \\
\hline
\end{tabular}

--TS with catalyst $\left(\mathrm{Ar}=2,4,6-\left({ }^{i} \operatorname{Pr}\right)_{3} \mathrm{C}_{6} \mathrm{H}_{2}\right)$ with phenyl group at the chiral carbon of substrate

$--$

Number of imaginary frequencies $=1$

The smallest frequency is $=-197.2980 \mathrm{~cm}(-1)$

Electronic energy $=\quad-3352.1015103\left(\mathbf{E}_{\text {sol }}=\mathbf{- 3 3 5 2 . 1 5 8 7 6 3 6}\right)$ 


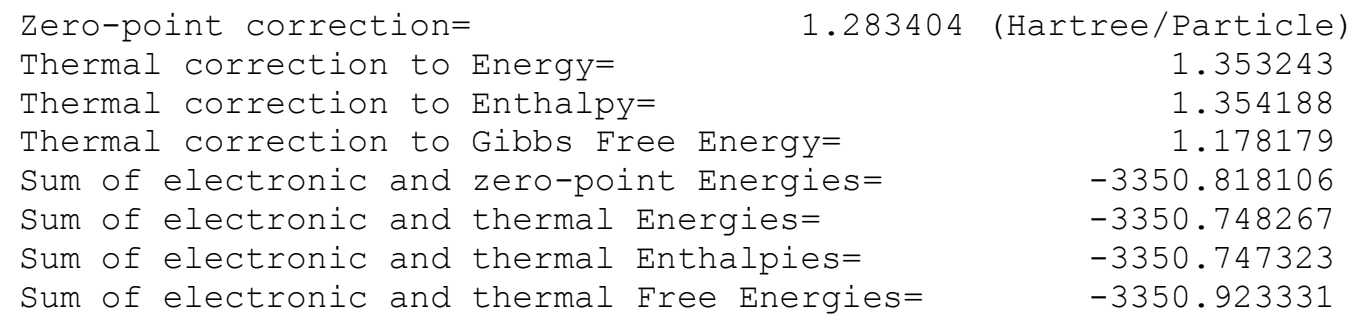

Cartesian Coordinates

\begin{tabular}{|c|c|c|c|}
\hline 6 & 5.226736 & 1.533464 & -1.230390 \\
\hline 6 & 4.305167 & 2.571949 & -0.932495 \\
\hline 6 & 3.109052 & 2.314484 & -0.312640 \\
\hline 6 & 2.879408 & 0.981057 & 0.120277 \\
\hline 6 & 3.728357 & -0.077229 & -0.124024 \\
\hline 6 & 4.922008 & 0.187707 & -0.875284 \\
\hline 1 & 4.532337 & 3.583700 & -1.258925 \\
\hline 6 & 3.750799 & -3.601496 & 1.436086 \\
\hline 6 & 2.462467 & -4.038039 & 1.030224 \\
\hline 6 & 1.594117 & -3.204814 & 0.370692 \\
\hline 6 & 2.070536 & -1.912515 & 0.024497 \\
\hline 6 & 3.331993 & -1.441735 & 0.328940 \\
\hline 6 & 4.185860 & -2.284907 & 1.114911 \\
\hline 1 & 2.152340 & -5.050909 & 1.271471 \\
\hline 6 & 6.231074 & -2.684781 & 2.363054 \\
\hline 6 & 5.439449 & -1.846305 & 1.620433 \\
\hline 1 & 7.182079 & -2.327406 & 2.744458 \\
\hline 1 & 5.762830 & -0.830972 & 1.419542 \\
\hline 6 & 5.815833 & -4.006780 & 2.645213 \\
\hline 6 & 4.600053 & -4.449416 & 2.194919 \\
\hline 1 & 6.454086 & -4.660134 & 3.230978 \\
\hline 1 & 4.256124 & -5.454462 & 2.423556 \\
\hline 6 & 6.943033 & -0.539289 & -2.011819 \\
\hline 6 & 5.801807 & -0.839032 & -1.311793 \\
\hline 1 & 7.598409 & -1.340143 & -2.338662 \\
\hline 1 & 5.558617 & -1.872434 & -1.091394 \\
\hline 6 & 7.268980 & 0.801799 & -2.319914 \\
\hline 6 & 6.422750 & 1.811096 & -1.942745 \\
\hline 1 & 8.177976 & 1.025478 & -2.868493 \\
\hline 1 & 6.644295 & 2.844373 & -2.195591 \\
\hline 8 & 1.201842 & -1.111882 & -0.694991 \\
\hline 8 & 1.732483 & 0.762827 & 0.857762 \\
\hline 15 & 0.466988 & 0.073761 & 0.130844 \\
\hline 8 & -0.387121 & -0.446113 & 1.263509 \\
\hline 8 & -0.195422 & 0.932341 & -0.914930 \\
\hline 1 & -1.731462 & -0.318277 & 1.022061 \\
\hline 6 & 0.196849 & -3.618115 & 0.024894 \\
\hline 6 & -0.755194 & -3.807534 & 1.048575 \\
\hline 6 & -0.167751 & -3.830536 & -1.320041 \\
\hline 6 & -2.023618 & -4.285342 & 0.706423 \\
\hline 6 & -1.452059 & -4.293198 & -1.609808 \\
\hline 6 & -2.390442 & -4.547179 & -0.610732 \\
\hline 6 & 2.005340 & 3.315480 & -0.189335 \\
\hline 6 & 1.349025 & 3.753759 & -1.360496 \\
\hline 6 & 1.553951 & 3.745106 & 1.071977 \\
\hline 6 & 0.275908 & 4.635348 & -1.238793 \\
\hline 6 & 0.467165 & 4.618530 & 1.139170 \\
\hline
\end{tabular}




\begin{tabular}{|c|c|c|c|}
\hline 6 & -0.190361 & 5.071078 & -0.000843 \\
\hline 8 & -2.744585 & -0.300481 & 0.677847 \\
\hline 1 & 0.117741 & 4.956789 & 2.113340 \\
\hline 1 & -0.224412 & 4.976379 & -2.142033 \\
\hline 1 & -1.719447 & -4.474367 & -2.649276 \\
\hline$\perp$ & -2.755770 & -4.449998 & 1.493704 \\
\hline 6 & 2.219745 & 3.317786 & 2.370922 \\
\hline 1 & 2.994930 & 2.581699 & 2.137307 \\
\hline 6 & 1.744736 & 3.293704 & -2.757711 \\
\hline 1 & 2.436830 & 2.453232 & -2.661427 \\
\hline 6 & -1.364990 & 6.024359 & 0.102671 \\
\hline 1 & -1.674068 & 6.044442 & 1.156287 \\
\hline 6 & 1.224943 & 2.652971 & 3.329857 \\
\hline 1 & 0.769717 & 1.772042 & 2.870130 \\
\hline 1 & 1.736320 & 2.337727 & 4.244897 \\
\hline 1 & 0.427693 & 3.347237 & 3.618907 \\
\hline 6 & 2.907497 & 4.515858 & 3.038210 \\
\hline 1 & 3.632762 & 4.978473 & 2.362896 \\
\hline 1 & 2.174225 & 5.279358 & 3.318491 \\
\hline 1 & 3.431034 & 4.202483 & 3.946693 \\
\hline 6 & 0.544431 & 2.789862 & -3.568006 \\
\hline 1 & -0.157569 & 3.598340 & -3.798099 \\
\hline 1 & 0.889847 & 2.378044 & -4.521561 \\
\hline 1 & 0.015308 & 2.008987 & -3.017887 \\
\hline 6 & 2.463871 & 4.420512 & -3.510157 \\
\hline 1 & 2.776976 & 4.084403 & -4.503520 \\
\hline 1 & 1.796768 & 5.280288 & -3.637298 \\
\hline 1 & 3.348944 & 4.766659 & -2.968395 \\
\hline 6 & -2.561377 & 5.554863 & -0.731816 \\
\hline 1 & -2.351661 & 5.645838 & -1.802797 \\
\hline 1 & -2.796947 & 4.507957 & -0.521123 \\
\hline 1 & -3.442464 & 6.167973 & -0.517063 \\
\hline 6 & -0.946895 & 7.444832 & -0.295645 \\
\hline 1 & -1.784987 & 8.141874 & -0.196307 \\
\hline 1 & -0.122315 & 7.801514 & 0.327434 \\
\hline 1 & -0.611910 & 7.462657 & -1.338278 \\
\hline 6 & -0.456193 & -3.498213 & 2.509566 \\
\hline 1 & 0.424748 & -2.850292 & 2.539543 \\
\hline 6 & 0.786058 & -3.583237 & -2.478745 \\
\hline 1 & 1.732387 & -3.209193 & -2.079158 \\
\hline 6 & -3.757026 & -5.116188 & -0.946364 \\
\hline 1 & -4.336280 & -5.144898 & -0.014603 \\
\hline 6 & -4.527674 & -4.256390 & -1.954578 \\
\hline 1 & -5.496589 & -4.712250 & -2.179786 \\
\hline 1 & -4.714683 & -3.247957 & -1.573677 \\
\hline 1 & -3.976895 & -4.166726 & -2.896679 \\
\hline 6 & -3.621724 & -6.554081 & -1.463188 \\
\hline 1 & -3.062419 & -6.571703 & -2.404567 \\
\hline 1 & -3.087245 & -7.180840 & -0.744583 \\
\hline 1 & -4.606001 & -6.995747 & -1.646515 \\
\hline 6 & 0.230524 & -2.513409 & -3.426803 \\
\hline 1 & -0.699874 & -2.849810 & -3.897200 \\
\hline 1 & 0.033149 & -1.582506 & -2.888088 \\
\hline 1 & 0.949532 & -2.303161 & -4.224452 \\
\hline 6 & 1.090725 & -4.885011 & -3.229699 \\
\hline 1 & 1.509785 & -5.637613 & -2.555854 \\
\hline 1 & 0.183925 & -5.303069 & -3.679043 \\
\hline 1 & 1.809839 & -4.702923 & -4.03403 \\
\hline
\end{tabular}




$\begin{array}{lrrr}6 & -0.157245 & -4.779375 & 3.298893 \\ 1 & 0.049078 & -4.543733 & 4.347555 \\ 1 & -1.019172 & -5.454942 & 3.266301 \\ 1 & 0.703934 & -5.317687 & 2.895760 \\ 6 & -1.601979 & -2.734670 & 3.185843 \\ 1 & -1.269613 & -2.355861 & 4.156940 \\ 1 & -1.922659 & -1.886467 & 2.578595 \\ 1 & -2.467091 & -3.381792 & 3.366320 \\ 6 & -2.790056 & -1.116702 & -0.503620 \\ 6 & -3.076975 & -0.168790 & -1.666661 \\ 1 & -3.597048 & -0.676596 & -2.482164 \\ 1 & -2.141866 & 0.247347 & -2.049091 \\ 6 & -3.951530 & 0.942253 & -1.060438 \\ 6 & -3.250752 & 1.363149 & 0.233475 \\ 1 & -1.359474 & 1.616265 & -0.407861 \\ 8 & -2.129032 & 2.036546 & 0.158190 \\ 1 & -1.837048 & -1.646882 & -0.613493 \\ 1 & -3.582399 & -1.855288 & -0.366054 \\ 8 & -4.079900 & 1.738440 & 1.187232 \\ 6 & -3.626132 & 2.270089 & 2.479115 \\ 6 & -2.574261 & 1.361596 & 3.103411 \\ 1 & -1.651262 & 1.346892 & 2.520407 \\ 1 & -2.336471 & 1.740916 & 4.101638 \\ 1 & -2.953157 & 0.340489 & 3.202041 \\ 6 & -4.914248 & 2.248288 & 3.293886 \\ 1 & -4.723563 & 2.628372 & 4.300667 \\ 1 & -5.675087 & 2.872786 & 2.818747 \\ 1 & -5.298757 & 1.227784 & 3.367128 \\ 6 & -3.117437 & 3.699477 & 2.315224 \\ 1 & -3.838644 & 4.294913 & 1.746990 \\ 1 & -3.006176 & 4.149293 & 3.307026 \\ 1 & -2.152322 & 3.724515 & 1.806579 \\ 1 & -3.928105 & 1.831180 & -1.699590 \\ 6 & -5.388077 & 0.485621 & -0.900981 \\ 6 & -6.302571 & 0.820152 & -1.901204 \\ 6 & -5.816720 & -0.328815 & 0.153664 \\ 6 & -7.613910 & 0.355268 & -1.859540 \\ 1 & -5.982180 & 1.452600 & -2.725606 \\ 6 & -7.127431 & -0.794235 & 0.195456 \\ 1 & -5.127187 & -0.580052 & 0.953614 \\ 6 & -8.030188 & -0.456480 & -0.809586 \\ 1 & -8.308642 & 0.630961 & -2.646603 \\ 1 & -7.445203 & -1.420911 & 1.022917 \\ 1 & -9.051967 & -0.820057 & -0.771243\end{array}$

